\title{
Collaborative care for depression and anxiety problems (Review)
}

\author{
Archer J, Bower P, Gilbody S, Lovell K, Richards D, Gask L, Dickens C, Coventry P
}

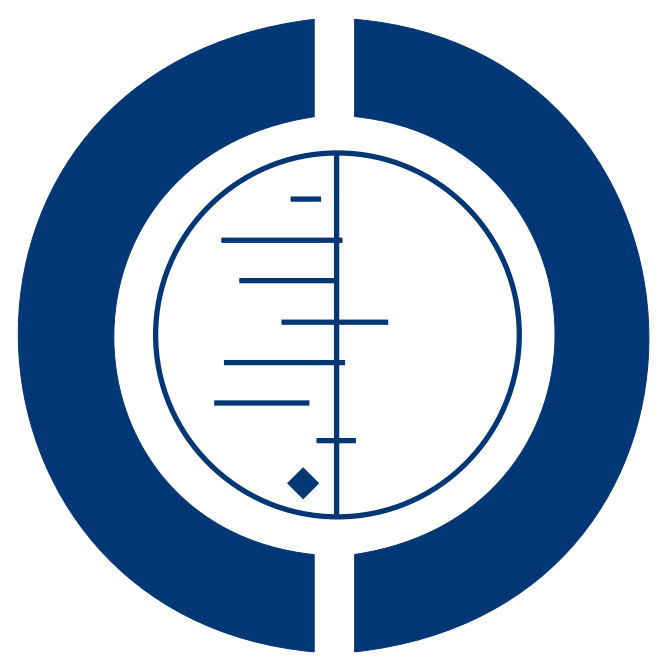

\section{THE COCHRANE COLLABORATION $^{\circledR}$}

This is a reprint of a Cochrane review, prepared and maintained by The Cochrane Collaboration and published in The Cochrane Library 2012, Issue 10

http://www.thecochranelibrary.com

\section{WILEY}


TABLE OF CONTENTS

HEADER . . . . . . . . . . . . . . . . . . . . . . . . . . . . . . . . . . . . . . . 1

ABSTRACT . . . . . . . . . . . . . . . . . . . . . . . . . . . . . . . . . . . . . . . . . . . .

PLAIN LANGUAGE SUMMARY . . . . . . . . . . . . . . . . . . . . . . . . . . . . . . . . . . . . . . . . .

BACKGROUND . . . . . . . . . . . . . . . . . . . . . . . . . . . . . . . . . . . . . .

OBJECTIVES . . . . . . . . . . . . . . . . . . . . . . . . . . . . . . . . . . . . . . . . 44

METHODS . . . . . . . . . . . . . . . . . . . . . . . . . . . . . . . . . . . . . . . . .

RESULTS . . . . . . . . . . . . . . . . . . . . . . . . . . . . . . . . . . . . . . . 9

Figure 1. . . . . . . . . . . . . . . . . . . . . . . . . . . . . 10

Figure 2. . . . . . . . . . . . . . . . . . . . . . . . . . . . . . . . . . . . . . .

Figure 3. . . . . . . . . . . . . . . . . . . . . . . . . . . . . . . . . . . . . . 16

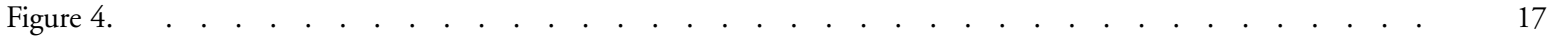

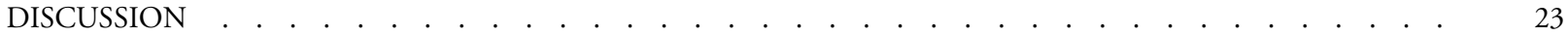

AUTHORS' CONCLUSIONS . . . . . . . . . . . . . . . . . . . . . . . . . . . . . . .

ACKNOWLEDGEMENTS . . . . . . . . . . . . . . . . . . . . . . . . . . . . . . . . . . . . .

REFERENCES . . . . . . . . . . . . . . . . . . . . . . . . . . . . . . . . . . . . . . 27

CHARACTERISTICS OF STUDIES . . . . . . . . . . . . . . . . . . . . . . . . . . . . . . 53

DATA AND ANALYSES . . . . . . . . . . . . . . . . . . . . . . . . . . . . . . . . . . . 224

HISTORY . . . . . . . . . . . . . . . . . . . . . . . . . . . . . . . . . . . . . . . 227

CONTRIBUTIONS OF AUTHORS . . . . . . . . . . . . . . . . . . . . . . . . . . . . . . . . . . . . . . . . . .

DECLARATIONS OF INTEREST . . . . . . . . . . . . . . . . . . . . . . . . . . . . . . . . . . .

SOURCES OF SUPPORT . . . . . . . . . . . . . . . . . . . . . . . . . . . . . . . . . . . 228

DIFFERENCES BETWEEN PROTOCOL AND REVIEW . . . . . . . . . . . . . . . . . . . . . . . . . . . . . . 229

INDEX TERMS . . . . . . . . . . . . . . . . . . . . . . . . . . . . . . . . . . . . 229 


\title{
[Intervention Review]
}

\section{Collaborative care for depression and anxiety problems}

\author{
Janine Archer $^{1}$, Peter Bower ${ }^{2}$, Simon Gilbody ${ }^{3}$, Karina Lovell $^{1}$, David Richards ${ }^{4}$, Linda Gask ${ }^{5}$, Chris Dickens ${ }^{6}$, Peter Coventry $^{7}$ \\ ${ }^{1}$ School of Nursing, Midwifery and Social Work, The University of Manchester, Manchester, UK. ${ }^{2}$ NIHR School for Primary Care \\ Research, Manchester Academic Health Science Centre, Institute of Population Health, University of Manchester, Manchester, UK. \\ ${ }^{3}$ Department of Health Sciences, University of York, York, UK. ${ }^{4}$ School of Psychology, University of Exeter, Exeter, UK. ${ }^{5}$ Health \\ Sciences Research Group, Manchester Academic Health Science Centre, University of Manchester, Manchester, UK. ${ }^{6}$ Institute of \\ Health Services Research, Peninsula College of Medicine and Dentistry, Exeter, UK. ${ }^{7}$ NIHR Collaboration for Leadership in Applied \\ Health Research and Care for Greater Manchester, Manchester Academic Health Science Centre, Institute of Population Health, \\ University of Manchester, Manchester, UK
}

Contact address: Janine Archer, School of Nursing, Midwifery and Social Work, The University of Manchester, Jean McFarlane Building (Room 6.304), Oxford Road, Manchester, Manchester, M13 9PL, UK. janine.archer@manchester.ac.uk.

Editorial group: Cochrane Depression, Anxiety and Neurosis Group.

Publication status and date: New, published in Issue 10, 2012.

Review content assessed as up-to-date: 9 February 2012.

Citation: Archer J, Bower P, Gilbody S, Lovell K, Richards D, Gask L, Dickens C, Coventry P. Collaborative care for depression and anxiety problems. Cochrane Database of Systematic Reviews 2012, Issue 10. Art. No.: CD006525. DOI: 10.1002/14651858.CD006525.pub2.

Copyright (C) 2012 The Cochrane Collaboration. Published by John Wiley \& Sons, Ltd.

\begin{abstract}
A B S T R A C T
Background

Common mental health problems, such as depression and anxiety, are estimated to affect up to $15 \%$ of the UK population at any one time, and health care systems worldwide need to implement interventions to reduce the impact and burden of these conditions. Collaborative care is a complex intervention based on chronic disease management models that may be effective in the management of these common mental health problems.
\end{abstract}

\section{Objectives}

To assess the effectiveness of collaborative care for patients with depression or anxiety.

\section{Search methods}

We searched the following databases to February 2012: The Cochrane Collaboration Depression, Anxiety and Neurosis Group (CCDAN) trials registers (CCDANCTR-References and CCDANCTR-Studies) which include relevant randomised controlled trials (RCTs) from MEDLINE (1950 to present), EMBASE (1974 to present), PsycINFO (1967 to present) and the Cochrane Central Register of Controlled Trials (CENTRAL, all years); the World Health Organization (WHO) trials portal (ICTRP); ClinicalTrials.gov; and CINAHL (to November 2010 only). We screened the reference lists of reports of all included studies and published systematic reviews for reports of additional studies.

\section{Selection criteria}

Randomised controlled trials (RCTs) of collaborative care for participants of all ages with depression or anxiety.

Collaborative care for depression and anxiety problems (Review)

Copyright $\odot 2012$ The Cochrane Collaboration. Published by John Wiley \& Sons, Ltd. 


\section{Data collection and analysis}

Two independent researchers extracted data using a standardised data extraction sheet. Two independent researchers made 'Risk of bias' assessments using criteria from The Cochrane Collaboration. We combined continuous measures of outcome using standardised mean differences (SMDs) with 95\% confidence intervals (CIs). We combined dichotomous measures using risk ratios (RRs) with $95 \%$ CIs. Sensitivity analyses tested the robustness of the results.

\section{Main results}

We included seventy-nine RCTs (including 90 relevant comparisons) involving 24,308 participants in the review. Studies varied in terms of risk of bias.

The results of primary analyses demonstrated significantly greater improvement in depression outcomes for adults with depression treated with the collaborative care model in the short-term (SMD - $0.34,95 \%$ CI -0.41 to -0.27 ; RR 1.32 , 95\% CI 1.22 to 1.43 ), medium-term (SMD - $0.28,95 \%$ CI -0.41 to -0.15 ; RR $1.31,95 \%$ CI 1.17 to 1.48 ), and long-term (SMD - $0.35,95 \%$ CI - 0.46 to 0.24; RR 1.29 , $95 \%$ CI 1.18 to 1.41 ). However, these significant benefits were not demonstrated into the very long-term (RR 1.12 , 95\% CI 0.98 to 1.27 ).

The results also demonstrated significantly greater improvement in anxiety outcomes for adults with anxiety treated with the collaborative care model in the short-term (SMD -0.30, 95\% CI -0.44 to -0.17; RR 1.50, 95\% CI 1.21 to 1.87), medium-term (SMD -0.33, 95\% CI -0.47 to -0.19 ; RR $1.41,95 \%$ CI 1.18 to 1.69 ), and long-term (SMD -0.20, 95\% CI -0.34 to -0.06 ; RR 1.26 , $95 \%$ CI 1.11 to 1.42). No comparisons examined the effects of the intervention on anxiety outcomes in the very long-term.

There was evidence of benefit in secondary outcomes including medication use, mental health quality of life, and patient satisfaction, although there was less evidence of benefit in physical quality of life.

\section{Authors' conclusions}

Collaborative care is associated with significant improvement in depression and anxiety outcomes compared with usual care, and represents a useful addition to clinical pathways for adult patients with depression and anxiety.

\section{PLAIN LANGUAGE SUMMARY}

\section{Collaborative care for people with depression and anxiety}

Many people suffer from depression and anxiety. These problems can make people feel sad, scared and even suicidal, and can affect their work, their relationships and their quality of life. Depression and anxiety can occur because of personal, financial, social or health problems.

'Collaborative care' is an innovative way of treating depression and anxiety. It involves a number of health professionals working with a patient to help them overcome their problems. Collaborative care often involves a medical doctor, a case manager (with training in depression and anxiety), and a mental health specialist such as a psychiatrist. The case manager has regular contact with the person and organises care, together with the medical doctor and specialist. The case manager may offer help with medication, or access to a 'talking therapy' to help the patient get better.

Collaborative care has been tested with patients in a number of countries and health care systems, but it is not clear whether it should be recommended for people with depression or anxiety.

In this review we found 79 randomised controlled trials (RCTs) (90 comparisons) including 24,308 patients worldwide, comparing collaborative care with routine care or alternative treatments (such as consultation-liaison) for depression and anxiety. There were problems with the methods in some of the studies. For example, the methods used to allocate patients to collaborative care or routine care were not always free from bias, and many patients did not complete follow-up or provide information about their outcomes. Most of the studies focused on depression and the evidence suggests that collaborative care is better than routine care in improving depression for up to two years. A smaller number of studies examined the effect of collaborative care on anxiety and the evidence suggests that collaborative care is also better than usual care in improving anxiety for up to two years. Collaborative care increases the number of patients using medication in line with current guidance, and can improve mental health related quality of life. Patients with depression and anxiety treated with collaborative care are also more satisfied with their treatment.

Collaborative care for depression and anxiety problems (Review) 


\section{B A C K G R O U N D}

\section{Description of the condition}

Common mental health problems, such as depression and anxiety, are highly prevalent with estimates of up to $15 \%$ of the UK population affected at any one time (NICE 2011a). The prevalence of individual common mental health disorders varies considerably. The one-week prevalence rates from the Office of National Statistics 2007 national survey were $4.4 \%$ for generalised anxiety disorder, 3.0\% for post-traumatic stress disorder (PTSD), 2.3\% for depression, $1.4 \%$ for phobias, $1.1 \%$ for obsessive compulsive disorder (OCD), and 1.1\% for panic disorder (McManus 2009). Worldwide, depression affects about 154 million people, and an estimated $5.8 \%$ of men and $9.5 \%$ of women will experience a depressive episode in any given year (WHO 2001a).

Depression and anxiety are a major cause of disease burden and disability (Ustun 2004) with depression projected to become one of the three leading causes of burden of disease by 2030 (Mathers 2006). Symptoms of depression include: depressed mood; loss of interest or pleasure in activities; insomnia or sleeping too much; and fatigue or loss of energy. Symptoms of anxiety differ but can include: excessive worry; feeling tense or restless; significant tension in muscles; and irritability (APA 2000). The impact of both disorders on social and occupational functioning, physical health and mortality is also substantial (Ormel 1999), and often anxiety and depression present together, disabling the person further (NICE 2011a). Depression also accounts for two-thirds of all suicides (Sartorius 2001).

Depression and anxiety are often chronic in nature, characterised by high rates of relapse and recurrence. Following their first episode of depression, at least $50 \%$ of people will go on to have one or more further episode(s), with the risk of relapse increasing to $70 \%$ after the second episode, and as high as $90 \%$ after a third episode (Kupfer 1991).

\section{Description of the intervention}

It is estimated that up to $90 \%$ of patients diagnosed with depression and anxiety are treated solely in primary care (NICE 2011a). However, the management of these disorders is often suboptimal (NHS 2002). The most common method of treatment for common mental health disorders in primary care is psychotropic medication (NICE 2011a). There are problems with this approach, as patients do not take the medication as prescribed for a variety of reasons including fears of addiction, dependency and side effects (Lingam 2002). Care for patients with chronic problems like depression is often not proactive; patients do not receive ongoing monitoring and care designed to reduce the burden of disorder and the likelihood of recurrence and relapse (Buszewicz 2011).
It has been recognised that improving the treatment of common mental health problems is a very complex task which requires changes to the way care is provided, together with additional resources to develop the appropriate systems to enable primary care professionals to deliver high quality care (Gilbody 2003a; Katon 1997; Katon 2001). Four distinct models of quality improvement in common mental health problems have been identified: training primary care staff, consultation-liaison, replacement/referral, and collaborative care (Bower 2005).

The collaborative care model is based on the principles of chronic disease management applied to conditions such as diabetes. The model can involve a large number of different interventions including: screening, education of patients, changes in practice routines, and developments in information technology (Wagner 1996). Collaborative care models are exemplars of 'complex interventions' which consist of a number of separate elements, where the particular elements that function as the 'active ingredient' can be difficult to identify (Medical Research Council 2008).

The term 'collaborative care' was first used to describe an intervention which was delivered by a primary care provider and a psychiatrist (Katon 1995a). However, there have been significant developments in the model since that time, and thus clear specification of the meaning of the term in line with current thinking is important. A widely accepted definition of collaborative care used in a systematic review of complex system interventions requires that four key criteria are met: a multi-professional approach to patient care, structured management plan, scheduled patient follow-ups, and enhanced inter-professional communication (Gunn 2006).

\section{How the intervention might work}

Research has suggested that a key aspect of effective collaborative care is 'case management' (Gilbody 2003a). Case management has been described as a health worker taking responsibility for proactively following up patients, assessing patient adherence to psychological and pharmacological treatments, monitoring patient progress, taking action when treatment is unsuccessful, and delivering psychological support (Von Korff 2001). Case managers work closely with the primary care provider (who retains overall clinical responsibility) and can receive regular supervision from a mental health specialist (Gilbody 2003a; Katon 2001).

\section{Why it is important to do this review}

Collaborative care is a model of care for common mental health problems which has generated worldwide interest in its effectiveness and cost-effectiveness. Although a number of reviews of collaborative care have been published, significant uncertainties remain. Many trials are from the United States, and their generalisability to other contexts and health care systems is unclear. Effectiveness may vary by patient population; collaborative care was not 
recommended by the National Institute for Clinical Excellence (NICE) for depression (NICE 2010) or anxiety (NICE 2011b), but was recommended for depression in patients with chronic disease (NICE 2009). The evidence base for collaborative care is also rapidly developing. Mental health policy in the UK highlights the importance of patient choice in treatments for mental health problems, and collaborative care could provide another option for services to complement other proven treatments. This review will consolidate the developing body of evidence on collaborative care and provide an up-to-date and rigorous assessment to inform policy and practice.

\section{O B J E C T I VES}

This review aims to evaluate the effectiveness of collaborative care for depression and anxiety.

\section{METHODS}

\section{Criteria for considering studies for this review}

\section{Types of studies}

We included all randomised controlled trials (RCTs), including cluster-RCTs.

\section{Types of participants}

Participant characteristics: Trial participants were either male or female patients of any age.

Diagnosis: Trial participants had a primary diagnosis of depression (including: acute, chronic, persistent, remitted, subthreshold and postnatal) or anxiety (including: generalised anxiety, panic, posttraumatic stress disorder (PTSD), phobias, social anxiety, health anxiety and obsessive compulsive disorder (OCD)). Diagnosis of trial participants was according to one of the following: 1) diagnosis made by primary care provider; 2) Research Diagnostic Criteria (RDC), Diagnostic and Statistical Manual (DSM) (APA 2000) or International Classification of Diseases (ICD) (WHO 1992) criteria; or 3) assessment through self-rated or clinician-rated validated instruments, e.g. Patient Health Questionnaire 9 (PHQ-9) (Kroenke 2001), Beck Depression Inventory (BDI) (Beck 1987) and/or Beck Anxiety Inventory (BAI) (Beck 1988). Some studies included a mixed population, of which only a proportion were depressed or anxious (e.g. where studies included a mix of patients who were at-risk drinking, suicidal or depressed). These were included only if the majority (>=50\%) of participants were depressed and/or anxious, to ensure that the results of the study related to our target group.
Comorbidity: Trial participants could also have long-term conditions (i.e. asthma, diabetes, chronic obstructive pulmonary disease), as well as a common mental health problem.

Setting: Trial participants could be identified in a variety of healthcare settings (excluding in-patient/specialist mental health), but the intervention had to be predominantly delivered in primary care or community settings.

\section{Types of interventions}

\section{Experimental intervention}

This review has adopted four key collaborative care criteria (Gunn 2006). We regarded studies as collaborative care studies if they fulfilled the following criteria.

1. A multi-professional approach to patient care. A primary care provider (general practitioner, family physician, primary care physician or a specialist providing undifferentiated medical care) and at least one other health professional (e.g. nurse, psychologist, psychiatrist, or pharmacist) or paraprofessional is involved with patient care. For the purposes of the current review, we characterised primary care as medical care involving first contact and ongoing care to patients, regardless of the patient's age, gender or presenting problem (Boerma 1999; WHO 2001b).

2. A structured management plan. Introduction of an organised approach to patient care including access to evidence based management information in the form of guidelines or protocols. Management included either or both pharmacological (e.g. antidepressant medication) and non-pharmacological interventions (e.g. patient and provider education, counselling, or cognitive behaviour therapy (CBT)).

3. Scheduled patient follow-ups. An organised approach to patient follow-up defined as one or more scheduled telephone or in-person follow-up appointments to provide specific interventions, facilitate treatment adherence, or monitor symptoms or adverse effects.

4. Enhanced inter-professional communication. Introduction of mechanisms to facilitate communication between professionals caring for the patient, including team meetings, case conferences, individual consultation/supervision, shared medical records, and patient-specific written or verbal feedback between care-givers.

\section{Comparator interventions}

We included studies that compared collaborative care with 'usual care' (for example, routine primary care, waiting lists, or untreated groups identified through screening) or collaborative care with other interventions.

Based on analysis of studies identified in the review, we distinguished the following three types of usual care. 
1. Studies that provided no additional intervention in the usual care group, including no notification of patient depression status.

2. Studies that provided additional interventions in the usual care group (such as education of primary care providers, or notification of patient depression status), but where these aspects of the intervention were applied to both arms, and potentially cancelled out.

3. Studies that enhanced usual care by providing an intervention that the collaborative care arm did not receive e.g. where only primary care clinicians in the usual care arm received training and educational materials on depression evaluation and treatment (Asarnow 2005).

Based on analysis of studies identified in the review, we distinguished the following three types of 'active comparisons'.

1. 'Alternative interventions' such as feedback alone, consultation-liaison and enhanced referral, which were compared with collaborative care.

2. 'Enhancements of collaborative care' such as collaborative care plus consultation-liaison, and collaborative care plus psychotherapy, which were compared with collaborative care.

3. 'Models of collaborative care interventions' such as collaborative care (medication) versus collaborative care (psychotherapy), which were compared directly.

\section{Types of outcome measures}

Where relevant (i.e. for the effects of collaborative care on depression) we reported both continuous and dichotomous outcomes. For dichotomous outcomes, studies generally reported either 'response' outcomes (i.e. a $\geq 50 \%$ reduction in symptom scores from baseline) or 'remission' (patients at each time point with scores under a particular threshold). For consistency, we reported response outcomes where possible.

\section{Primary outcomes}

Change in depression or anxiety, as measured by observer or patient self-report.

\section{Secondary outcomes}

- Medication for depression and/or anxiety. This was reported as the proportion of patients using medication, proportions meeting predefined levels of use, or proportions with 'appropriate' use according to guidelines or other measures. Such data could be based on administrative data or patient self-report. We pooled data relating to rates of use and adherence, and administrative data and self-report.

We included the following outcomes only when a validated tool was used.

- Social functioning, e.g. Social Adaptation Self-evaluation Scale (SASS) (Bosc 1997).
- Quality of life, e.g. Short Form Health Survey (SF-36, SF12) (Ware 1993).

- Patient satisfaction, e.g. Client Satisfaction Questionnaire (CSQ) (Attkinson 2003).

\section{Timing of outcome assessment}

We categorised outcomes as short-term (0 to 6 months), mediumterm (7 to 12 months), long-term (13 to 24 months), and very long-term (25 months or more). We rounded down studies that reported unconventional follow-up points (e.g. 27 weeks).

\section{Search methods for identification of studies}

\section{CCDAN's Specialised Register}

The Cochrane Collaboration Depression, Anxiety and Neurosis Group (CCDAN) maintain two clinical trials registers at their editorial base in Bristol, UK; a references register and a studiesbased register. The CCDANCTR-References Register contains over 29,500 reports of trials in depression, anxiety and neurosis. Approximately $65 \%$ of these references have been tagged to individual, coded trials. The coded trials are held in the CCDANCTRStudies Register and records are linked between the two registers through the use of unique Study ID tags. Coding of trials is based on the EU-Psi coding manual. Further details are available from the CCDAN Trials Search Co-ordinator (TSC). Reports of trials for inclusion in the registers are collated from routine (weekly) generic searches of MEDLINE (1950 to present), EMBASE (1974 to present), and PsycINFO (1967 to present); quarterly searches of the Cochrane Central Register of Controlled Trials (CENTRAL); and review specific searches of additional databases. Reports of trials are also sourced from international trials registers c/o the World Health Organization's (WHO's) trials portal (ICTRP) (http:// apps.who.int/trialsearch/), drug companies, the handsearching of key journals, conference proceedings, and other (non-Cochrane) systematic reviews and meta-analyses.

Details of CCDAN's generic search strategies can be found on the Group's website.

\section{Electronic searches}

We searched the CCDAN registers (to 9th February 2012) using the following terms.

\section{CCDANCTR-Studies}

Condition $=\left(\right.$ depress $^{*}$ or dysthymi ${ }^{*}$ or anxiety or anxious or panic or *phobi* or obsessi* or compulsi* or post-traumatic) and Intervention $=$ ("care manag*” or "case manage ${ }^{*}$ " or collaborat* or "disease manag*“ or "enhanced care" or "managed care" or multicomponent or multi-component or multidisciplinary or multidisciplinary or stepped)

\section{CCDANCTR-References}

Collaborative care for depression and anxiety problems (Review) 
The CCDANCTR-References Register was searched using a more sensitive set of terms to identify additional untagged/uncoded references:

1. (depress* or dysthymi* or anxiety or anxious or * ${ }^{*}$ hobi* or PTSD or post-trauma* or "post trauma*" or postrauma* or panic or OCD or obsessi* or compulsi* or GAD) [ti, ab, kw]

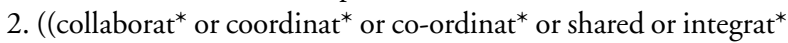
or stepped or systematic) AND (care or healthcare or "health care" or working or intervention* or service or model or effort* or manage*)) [free-text $]$

3. ((augment* ${ }^{*}$ or enhance*) AND (care* or healthcare or "health care" or communicat $\left.{ }^{*}\right)$ [free-text]

4. ("care manage"” or "case manage*" or "chronic care*" or "complex intervention*" or "cooperative behav" or "co-operative behav"” or "joint working" or pathway or interprofessional or inter-professional or interdisciplinary or inter-disciplinary or multidisciplin* or multi-disciplin* or multiprofession* or multi-profession* or transdisciplin* or trans-disciplin* or multifacet* or multifacet* or "complex intervention*" or "multiple intervention*" or multi-intervention* or "organisational intervention*" or "organizational intervention*" or "interpersonal relation*" or " inter-personal relation*" or "interinstitutional relation*" or "inter-institutional relation*" or "consultation liaison" or algorithm* or "treatment guideline*" or "treatment protocol*" or "treatment delivery" or "treatment model" or adherence or compliance or concordance or "patient care team" or "patient care management" or "patient care planning" or "case management" or "managed care program*" or "delivery of healthcare" or "continuity of patient care" or "professional-patient relations" or "interprofessional relations") [freetext]

5. (1 and $(2$ or 3 or 4$))$

3. CINAHL (1982 to 11th November 2010)

We conducted an additional search on CINAHL (Cumulative Index to Nursing and Allied Health) (search strategy in Appendix $1)$.

\section{International Trial Registers}

We also carried out searches on the WHO trials portal (ICTRP) and ClinicalTrials.gov to identify ongoing or unpublished studies using the terms:

("stepped care" or "collaborative care" or interprofessional or interdisciplinary or multidisciplinary). We imported and filtered results into Excel using terms for depression and anxiety.

\section{Searching other resources}

We checked the reference lists of reports of all included studies and other systematic reviews for additional published, unpublished or ongoing research.

\section{Data collection and analysis}

\section{Selection of studies}

Two review authors (JA and PB) independently scanned the identified studies and excluded studies according to the criteria above, on the basis of titles and abstracts. We retrieved full copies of the studies deemed eligible by one of the team (JA) for closer examination. If there was uncertainty or disagreement, we reached consensus by discussion and consultation with another review author $(\mathrm{PB}$, DR or SG). A log of all studies which initially appeared to meet the inclusion criteria but which we later excluded on retrieval of the full-text are detailed in the Characteristics of excluded studies tables. We kept a record of the reasons for exclusion.

\section{Data extraction and management}

Content data were extracted by JA, DR, KL and LG and doubleextracted by research assistants/associates. Outcome data were extracted by $\mathrm{PB}$ and research assistants. A standardised data extraction form was used for the following characteristics.

1. The patient population (demographic and clinical characteristics).

2. The nature of the intervention (e.g. types of interventions used, contact between patient and professional, and amount of collaboration between professionals).

3. Internal validity (assessment of risk of bias).

4. External validity (context of recruitment and methods of recruitment).

We presented analyses using the following structure. In the analysis of primary outcomes we distinguished all collaborative care interventions, separating studies by diagnosis (depression and anxiety) and age (adolescents and adults). Therefore analyses 1.1, 1.2 and 1.3 report outcomes for depression in adults, analyses 1.4, 1.5 and 1.6 report outcomes for anxiety in adults and analyses 2.1, 2.2 and 2.3 report outcomes for depression in adolescents. No studies reported anxiety outcomes in adolescents.

We separately analysed primary outcomes reported as dichotomous outcomes and as continuous outcomes. Each type of outcome was reported at four time periods: 0 to 6 months, 7 to 12 months, 13 to 24 months, and $25+$ months.

For the secondary outcome of medication use, we applied the same analytical methods. The majority of studies reported medication use using dichotomous outcomes; we excluded the minority reporting continuous outcomes.

For the secondary outcome of quality of life, we combined analyses across collaborative care interventions for patients with depression and anxiety. The majority of studies reported quality of life using continuous outcomes; we excluded the minority reporting dichotomous outcomes. We split quality of life outcomes into mental health quality of life (e.g. SF-36 emotional role, SF-mental component score), and physical health quality of life (e.g. SF-36 physical functioning, SF-physical component score). We excluded measures that did not report separate mental health and physical health dimensions (e.g. EQ5D overall utility). 
For satisfaction outcomes, we combined analyses across collaborative care intervention for patients with depression and anxiety. We analysed satisfaction outcomes reported as dichotomous outcomes and continuous outcomes separately. We only reported a single satisfaction outcome point for each study, choosing the outcome closest to six months as the likely best indicator of patient experience of the intervention, unaffected by memory or other bias.

As part of the protocol, we intended to report on social function outcomes. However, a very wide variety of social function outcome measures were reported, and there was a lack of clarity over their definition, scope, and comparability. It was therefore not possible to produce a rigorous synthesis in the time frame of the review. We have extracted social function outcomes and may report on these in a later update of the review when a suitable typology has been developed to ensure consistency in analysis.

\section{Assessment of risk of bias in included studies}

For each included study, one review author (JA, PC, CD or DR) and one research assistant/associate independently applied The Cochrane Collaboration's 'Risk of bias' tool (Higgins 2011b). This tool encourages consideration of:

1. selection bias due to inadequate generation of a randomised sequence;

2. selection bias due to inadequate concealment of allocations prior to assignment;

3. performance bias due to knowledge of the allocated interventions by participants and personnel during the study (blinding);

4. detection bias due to knowledge of the allocated interventions by outcome assessors (blinding);

5. attrition bias due to amount, nature or handling of incomplete outcome data;

6. reporting bias due to selective outcome reporting;

7. bias due to integrity of the intervention; and

8. bias due to other problems, such as:

- any potential source of bias related to the specific study design used; or

- claims to have been fraudulent; or

- some other problem.

We used our comments to show how we assessed the risk of bias, with judgements of either low risk of bias, unclear risk of bias, or high risk of bias. If there was uncertainty or disagreement, we reached consensus by discussion and consultation with another review author (PC).

\section{Measures of treatment effect}

Studies in the review reported both dichotomous (e.g. recovered/ not recovered) and continuous outcomes (such as patient scores on self-reported outcome scales). For dichotomous outcomes, we calculated risk ratios (RRs) and 95\% confidence intervals (CIs).
For continuous outcomes, as a range of different measures were used, we calculated standardised mean differences (SMDs) and $95 \%$ CIs.

\section{Unit of analysis issues}

\section{Cluster-randomised controlled trials}

As collaborative care is an organisational intervention, cluster trials are commonly used as a way of avoiding bias associated with contamination. We identified studies using cluster randomisation and we adjusted the precision of analyses based on these studies in the meta-analysis using the 'effective sample size' method outlined in the Cochrane Handbook for Systematic Reviews of Interventions (section 16.3.4) (Higgins 2011a). We calculated the effective sample size of groups in each cluster trial on the basis of the original sample size divided by the 'design effect'. The design effect was calculated by $1+(M-1)$ ICC, where $M$ represents the average cluster size and ICC is the intracluster correlation coefficient. We assumed a common design effect across groups. For the base analysis we assumed an intra-class correlation of 0.02 (Adams 2004). We examined the effect of adjustment for clustering in a sensitivity analysis using intra-class correlations of 0.00 and 0.05 (Donner 2002).

\section{Studies with multiple treatment groups}

Where studies reported multiple collaborative care interventions against a single control we extracted each collaborative care intervention as a separate comparison and entered them where relevant in the meta-analysis, dividing the control group sample size appropriately to avoid double-counting in the analysis. Where a study reported a single collaborative care intervention against two different types of controls (individual and cluster controls) we treated this as two separate comparisons, dividing the intervention group sample size to avoid double-counting in the analysis.

\section{Dealing with missing data}

We distinguished between two types of 'loss' of data: patients who did not complete their assigned collaborative care treatment ('treatment completion') and patients who did not complete follow-up for assessment of outcome ('loss to follow-up').

For 'treatment completion', we assessed whether the study used an appropriate 'intention-to-treat' analysis (including all patients in the analysis irrespective of treatment completion) or 'per protocol' analysis (excluding patients who did not complete treatment according to some defined criterion). We describe the approaches used by individual studies in Characteristics of included studies. To assess 'loss to follow-up' in included studies, we also calculated the proportion of randomised patients who were lost to followup at the 0 to 6 month follow-up across arms, and within each 
arm, and also calculated the difference in the proportions between collaborative care and usual care arms.

Data for the meta-analysis were missing for many outcomes, usually in terms of missing standard deviations (SDs) and sample sizes. In a change from the study protocol, we did not contact all authors to collect missing data as it was not possible to complete this task in the time available for the review. We did contact two authors for data in order to allow us to include their studies in the review (McCusker 2008; Rost 2001a; Rost 2001b) as the data reported in the published papers was not in the form required. We did not impute missing data required for calculations of treatment effect (e.g. missing SDs), but we did recalculate necessary parameters from published data (e.g. calculating SDs from published standard errors). When we update the review we will impute data for metaregression analysis to maximise the numbers of studies available for the analysis.

\section{Assessment of heterogeneity}

We examined heterogeneity using the $\mathrm{I}^{2}$ statistic, an estimate of the percentage of total variation across studies that can be attributed to heterogeneity rather than chance. This statistic is interpreted as follows: $0 \%$ to $40 \%$ might not be important, $30 \%$ to $60 \%$ might represent moderate levels of heterogeneity, $50 \%$ to $90 \%$ might represent substantial levels of heterogeneity, and $75 \%$ to $100 \%$ considerable heterogeneity (Deeks 2011). We calculated the 95\% confidence intervals around the $\mathrm{I}^{2}$ estimate using the Stata command heterogi. In the original protocol, we planned to use a random-effects model where a moderate to high $(50 \%$ or more) level of statistical heterogeneity was found (Higgins 2003). However, given the high levels of clinical and methodological heterogeneity in terms of participants, interventions, comparisons and outcome measures (see Characteristics of included studies), we used random-effects models in all analyses.

\section{Assessment of reporting biases}

We examined funnel plots to test for asymmetry which can indicate a number of issues including: selection bias (such as publication bias), poor methodological quality, and true heterogeneity (Egger 1997). We also reported any instances of selective outcome reporting in the 'Risk of bias' assessment.

\section{Data synthesis}

We used a random-effects model for all meta-analyses.

\section{Subgroup analysis and investigation of heterogeneity}

From previous analyses we expected heterogeneity in terms of treatment effects across different populations and types of interventions and we planned to examine these. Our primary analysis was collaborative care versus usual primary care. Other planned secondary analyses were to examine comparisons of different study designs, participants and types of collaborative care. This would include:

- types of participants

$\circ$ country (United States, other)

- location of recruitment (primary care, community, specialist, mixed); and location of delivery (primary care, community, specialist, mixed)

○ ethnicity ( $75 \%$ or more white, other)

- baseline severity (subthreshold, met criteria for major depressive or anxiety disorder, mixed)

- the complexity of the intervention

- types of professionals (primary care provider and case manager, or primary care provider, case manager and mental health specialist)

$\circ$ intervention intensity (measures of sessions, and sessions multiplied by session length)

- intervention content (medication management alone, psychological intervention alone, and combined).

We had planned to undertake a series of exploratory analyses using meta-regression, to examine the influence of these and other study-level factors in predicting the magnitude and direction of outcomes (Thompson 2002). We had planned to assess the significance of predictive factors (selected a priori and outlined above) in explaining between-study heterogeneity, as measured by the $\mathrm{I}^{2}$ statistic, according to the method proposed in (Higgins 2004).

We did not undertake these further exploratory analyses due to time constraints, but it is envisaged that we will include them in the review update.

\section{Sensitivity analysis}

We conducted sensitivity analyses to assess the effects of excluding certain types of studies: cluster trials; trials including patients on the basis of comorbid physical conditions; studies considered at high risk of bias based on concealment of allocation methods and attrition (studies with $>20 \%$ loss to follow-up). We conducted these sensitivity analyses only on depression outcomes (both continuous and dichotomous) at six months.

Following review, we also conducted a posthoc sensitivity analysis on intervention length. Our analysis of outcomes was based on time since randomisation ( 0 to 6 months, 7 to 12 months, 13 to 24 months, $25+$ months), but some collaborative care interventions continue for periods of greater than six months, and it is possible that the longer-term effects of collaborative care (i.e. those in the 7- to 12-month period and beyond) do not reflect any enduring effect of the intervention, but simply reflect those interventions that are extended beyond the initial outcome period ( 0 to 6 months). To assess this possibility, we coded studies as to whether the intervention is completed in the 0 - to 6 -month outcome point, or extended beyond that. In a sensitivity analysis, we removed those studies where the intervention extended beyond six months, to assess whether the effects found at the 7- to 12-month 
time point were significantly different when studies with longerterm interventions were excluded.

\section{R E S U L T S}

\section{Description of studies}

See: Characteristics of included studies; Characteristics of excluded studies; Characteristics of studies awaiting classification; Characteristics of ongoing studies.
See: Characteristics of included studies; Characteristics of excluded studies; Characteristics of studies awaiting classification; Characteristics of ongoing studies

\section{Results of the search}

After removal of duplicates, we identified 3473 references from the searches. After assessing the titles and abstracts we checked 618 full-texts, and included 79 randomised controlled studies $(90$ individual comparisons) in the review (435 references; nine studies had multiple comparisons) (see flow diagram in Figure 1). 
Figure I. Study flow diagram.

3620 records identified through database searching
44 additional records identified through other sources (14 were studies that were identified in an earlier review of collaborative care or through separate searches of databases/journals; 30 were companion papers to studies found by the search strategy)

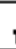

3473 records after duplicates removed
2855 records

excluded

183 full-text articles excluded:

27 did not meet study design criteria

97 did not meet intervention criteria

18 did not meet diagnostic criteria

41 were companion papers of studies excluded above 


\section{Included studies}

We included 79 randomised controlled trials (RCTs) (90 comparisons) involving 24,308 participants in the review.

The 'Characteristics of included studies' table details the characteristics of the studies, including study design, the characteristics of participants, the characteristics of interventions and outcome measures. These are summarised for the 90 comparisons below (figures are rounded to nearest whole numbers, and so the overall percentage does not always equal 100).

\section{Design}

All included comparisons were RCTs; 21 (23\%) comparisons used cluster randomisation, where the unit of randomisation was either a primary care practice $(n=19)$ or a primary care provider $(n=2)$.

\section{Setting}

Sixty-eight comparisons (76\%) were conducted in the US; 10 $(11 \%)$ in the UK; five $(6 \%)$ in other European countries (Germany, The Netherlands); and seven (8\%) from other countries (Canada, Chile, India, Puerto Rico).

Sixty-nine comparisons (77\%) recruited participants from primary care; eight (9\%) from community settings; 11 (12\%) from specialist physical health settings; and two (2\%) used a mixture of primary/community/specialist settings.

\section{Participants}

Participant characteristics: Seventy-nine comparisons (88\%) focused on adults aged 18 to 64 years; two (2\%) on adolescents under the age of 18 ; and nine (10\%) on those 65 years or more. For comparisons with available data $(n=70), 33(47 \%)$ included a sample of predominately white origin (classed as $75 \%$ or more of the sample). Twenty-one comparisons (23\%) included only those who were taking medication for depression and/or anxiety at baseline.

Diagnosis: Eighty-four comparisons (93\%) included participants with symptoms of depression or depression and anxiety; six (7\%) included only participants with anxiety disorders.

The diagnostic status of participants was identified in 45 comparisons (50\%) using Research Diagnostic Criteria (RDC), Diagnostic and Statistical Manual (DSM) (APA 2000) or International Classification Disorder (ICD) (WHO 1992) criteria. In the remainder, depression or anxiety status at point of entry was defined by self-rated or clinician-rated validated instruments or by the primary care provider without the use of standardised measures or criteria. In three comparisons (3\%) participants did not have to have symptoms of depression at baseline (Bartels 2004; Kroenke
2010; Williams 2007). As stated in the protocol, we included these studies since at least $50 \%$ of participants had depression at baseline, based on mean score of depression outcome measure or numbers provided.

Sixty-five comparisons (72\%) included participants with both subthreshold and diagnosed major depressive or anxiety disorder; 23 (26\%) included only those that met diagnostic criteria for major depressive or anxiety disorder; and two (2\%) included only subthreshold patients.

Sixteen comparisons (18\%) had physical comorbidity as an inclusion criteria, such as, diabetes (Bogner 2010; Ell 2010; Katon 2004; Piette 2011), cancer (Dwight-Johnson 2005; Ell 2008; Kroenke 2010; Strong 2008), epilepsy (Ciechanowski 2010), poststroke (Williams 2007), heart disease (Huffman 2011; Rollman 2009) or other/mix of conditions (Bogner 2008; Katon 2010; Pyne 2011; Vera 2010).

Setting: In 82 comparisons (91\%) the main healthcare provider was based in primary care; in eight comparisons (9\%) a specialist provided general medical care.

\section{Interventions}

All comparisons had to meet the four criteria of collaborative care stated in the protocol although there was considerable variability in the exact nature of the intervention.

- A multi-professional approach to patient care: all comparisons involved a primary care provider (generic medical professional) and at least one other health professional (e.g. psychiatrist, nurse, psychologist). In 78 comparisons (87\%) the intervention involved contributions from people with three distinct roles (primary care provider, case manager, mental health specialist); 12 (13\%) involved two professional roles (primary care provider and case manager, although in these comparisons typically the case manager was a mental health specialist). In 50 comparisons $(56 \%)$ the case manager was a mental health practitioner; in $40(44 \%)$ the case manager did not have a professional background in mental health.

- A structured management plan: all comparisons included an organised approach to patient care (e.g. evidence based medication algorithm, manualised psychological interventions such as behavioural activation or cognitive behaviour therapy (CBT)). In 48 comparisons (53\%) the intervention included medication management and psychological therapy; 37 (41\%) included medication management only; and 5 (6\%) psychological therapy only.

- Scheduled patient follow-ups: all comparisons included an organised approach to patient follow-up (e.g. scheduled telephone or in-person follow-up appointments). In 49 (54\%) of 
the comparisons the intervention lasted six months or less, in 31 (34\%) comparisons the intervention lasted more than six months, and it was unclear how long the intervention lasted in $10(11 \%)$ comparisons.

- Enhanced inter-professional communication: all comparisons introduced mechanisms to facilitate communication between professionals (e.g. team meetings, individual consultation/supervision, shared medical records, and patient-specific written or verbal feedback between care-givers).

The duration of the intervention varied across studies and data extraction was complex. Detailed data were not always reported, and the intensity of collaborative care interventions is sometimes contingent on short-term outcomes rather than being standardised for all patients, and may be titrated over time so that an initial high intensity intervention is replaced by low intensity monitoring over the longer-term. We estimated that 32 comparisons (36\%) included an intervention of more than six months duration. We will explore variability between studies in meta-regression analyses and include this in the updated review.

\section{Comparison group}

Thirty-four (38\%) comparisons provided no additional intervention in the usual care group. Fifty-two (58\%) comparisons did provide additional interventions in the usual care group (such as education (guidelines or brief training session) for primary care providers on the recognition and management of depression, or notification of patient's depression status) but these aspects of the intervention were also applied in the intervention arm. One (1\%) comparison enhanced usual care by providing an intervention that the collaborative care arm did not receive (Asarnow 2005). One (1\%) comparison did not describe usual care (Uebelacker 2011).

\section{Excluded studies}

Of the 3473 records screened, we excluded 2855 (82\%) on title and abstract. We retrieved 618 full-text articles and excluded 183 (30\%) from the review. Of these, 27 did not meet study design criteria (e.g. not RCTs), 97 did not meet intervention criteria (e.g. the intervention was not focused on the depression or anxiety, only included one professional, did not include enhanced communication or scheduled follow-ups), 18 did not meet diagnostic criteria (e.g. less than $50 \%$ of participants were depressed or anxious at baseline), and 41 were companion papers of the excluded ones. The 'Characteristics of excluded studies' table lists those trials which were potentially relevant $(n=37)$ but which did not meet all the inclusion criteria for the review, together with the exact criteria on which they were excluded. We excluded 24 because of the type of intervention used, 11 because of the types of participants included, and two because of study design.

\section{Ongoing studies}

Twenty studies are classified as 'ongoing' (Characteristics of ongoing studies). We contacted all lead authors of these studies, and whilst some studies were complete, data were not published/ available in time to include in the review.

\section{Studies waiting classification}

Eight studies are awaiting classification because we either have not been able to contact authors/are awaiting author response, the study is completed and we are awaiting publication of results, or translation was not possible within the time frame of the review (Characteristics of studies awaiting classification).

\section{Risk of bias in included studies}

A graphical representation of the risk of bias in included studies is presented in Figure 2. 
Figure 2. 'Risk of bias' summary: review authors' judgements about each risk of bias item for each included study.

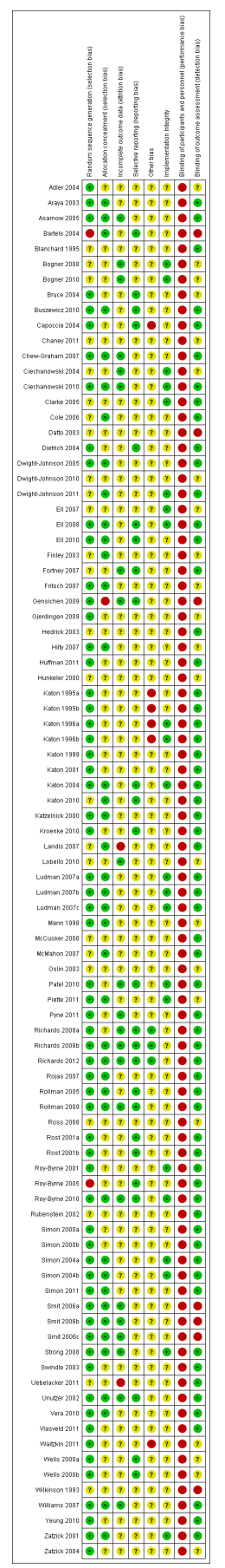




\section{Allocation}

\section{Generation of random sequence}

In sixty-three $(70 \%)$ comparisons random sequence generation was described adequately and we rated these as 'low risk' of bias. In twenty-five (28\%) comparisons the description of how the sequence was generated was either missing or there was insufficient information available to make an assessment and we rated these as 'unclear risk' of bias. Two (2\%) comparisons described methods which were considered to be at 'high risk' of bias (Bartels 2004; Roy-Byrne 2005).

\section{Allocation}

In forty (44\%) comparisons there was adequate description of allocation concealment and we rated these as 'low risk' of bias. In forty-nine (54\%) comparisons the description of allocation concealment was either missing or there was insufficient information available for assessment and we rated these as 'unclear risk' of bias. One (1\%) comparison described methods which were considered to be at 'high risk' of bias (Gensichen 2009).

\section{Blinding}

Blinding of participants and personnel was not possible in any case. We therefore rated all comparisons at 'high risk' of bias in relation to this criterion.

Sixty-one (68\%) comparisons described adequate blinding of those completing outcome assessment and we rated these at 'low risk' of bias. In twenty-two (24\%) comparisons the description of blinding of outcome assessment was either missing or there was insufficient information available for assessment and we rated these as 'unclear risk' of bias. Seven (8\%) comparisons described methods which we considered to be at 'high risk' of bias (Bartels 2004; Datto 2003; Gensichen 2009; Smit 2006a; Smit 2006b; Smit 2006c; Wilkinson 1993).

\section{Incomplete outcome data}

In terms of the proportion of randomised patients who were lost to follow-up at the 0 to 6 month follow-up, for the 87 comparisons where rates could be calculated, $26(30 \%)$ had $10 \%$ or less loss to follow-up, 38 (44\%) had $11 \%$ to $20 \%, 14$ (16\%) had $21 \%$ to $30 \%, 6(7 \%)$ had $31 \%$ to $40 \%$, and $3(3 \%)$ had $40 \%$ or more loss to follow-up.

In terms of differences in the proportions between collaborative care and usual care arms, seven (8\%) comparisons had differences of greater than $10 \%$ between trial arms.
Twenty-three (26\%) comparisons did not have high rates of loss to follow-up or imbalance and described adequate methods of dealing with incomplete outcome data and we rated these as 'low risk' of bias. In sixty-six (73\%) comparisons the rates of loss to follow-up or imbalance were high, the description of methods for dealing with incomplete outcome data was missing, or there was insufficient information available for assessment, and we rated these as 'unclear risk' of bias. One (1\%) comparison had high rates of loss to follow-up and described methods of dealing with missing data which were considered to be at 'high risk' of bias (Uebelacker 2011).

\section{Selective reporting}

In twenty-five comparisons (28\%) the authors had made protocols available and reported on all expected outcomes, therefore we rated these as 'low risk' of bias. Sixty-five comparisons (72\%) did not have a protocol available and/or insufficient information was available to judge selective reporting, and we rated these as 'unclear risk' of bias.

\section{Other potential sources of bias}

Using the three criteria to assess other potential sources of bias: 1) any potential source of bias related to the specific study design used; 2) study claimed to have been fraudulent; or 3) some other problem, we rated 81 comparisons $(90 \%)$ as 'unclear risk' of bias, three $(3 \%)$ as 'low risk' of bias and six $(7 \%)$ as 'high risk' of bias. We made the high risk of bias judgements based on analytical methods used or cross-contamination, where case managers were specified to provide care for patients in both usual care and collaborative care groups.

\section{Effects of interventions}

\section{Collaborative care versus usual care (adults)}

\section{I.I and I.2 Depression}

\section{Short-term: 0 to 6 months}

Thirty comparisons (5984 participants) reported short-term continuous outcomes for depression for collaborative care versus usual care. Collaborative care was significantly more effective than usual care (standard mean difference (SMD) $-0.34,95 \%$ CI -0.41 to $0.27, \mathrm{I}^{2}=34 \%$ ) (Analysis 1.1). 
Forty-eight comparisons (11,250 participants) reported shortterm dichotomous outcomes for depression for collaborative care versus usual care. Collaborative care was significantly more effective than usual care (risk ratio (RR) 1.32, 95\% CI 1.22 to 1.43 , $\mathrm{I}^{2}=71 \%$ ) (Analysis 1.2).

The funnel plots for the analyses of short-term continuous and dichotomous outcomes are shown in Figure 3 and Figure 4. Neither showed marked evidence of asymmetry. 
Figure 3. Forest plot of comparison: I Collaborative care versus 'usual care' (adults), outcome: I.I Improvement in depression symptoms.

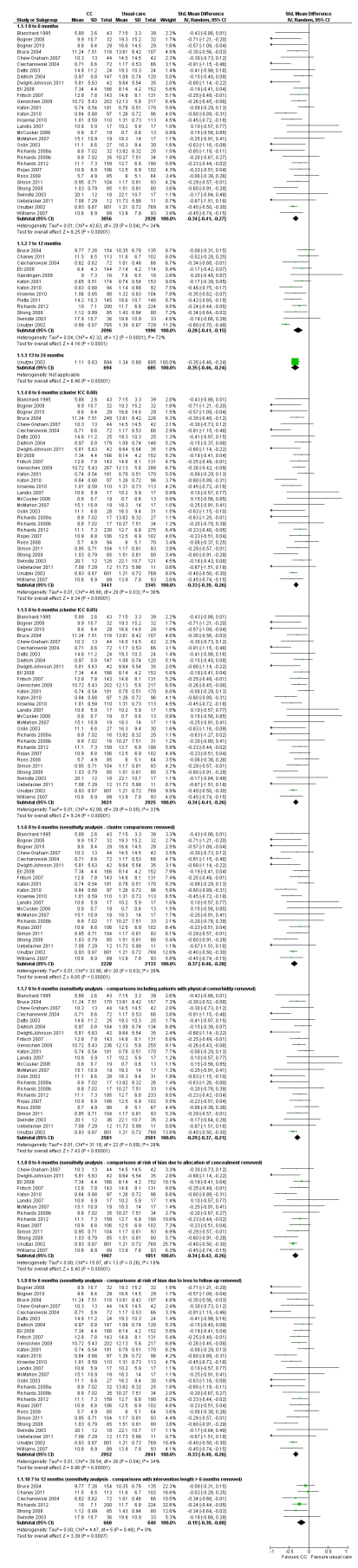


Figure 4. Forest plot of comparison: I Collaborative care versus 'usual care' (adults), outcome: I.2 Depression response.

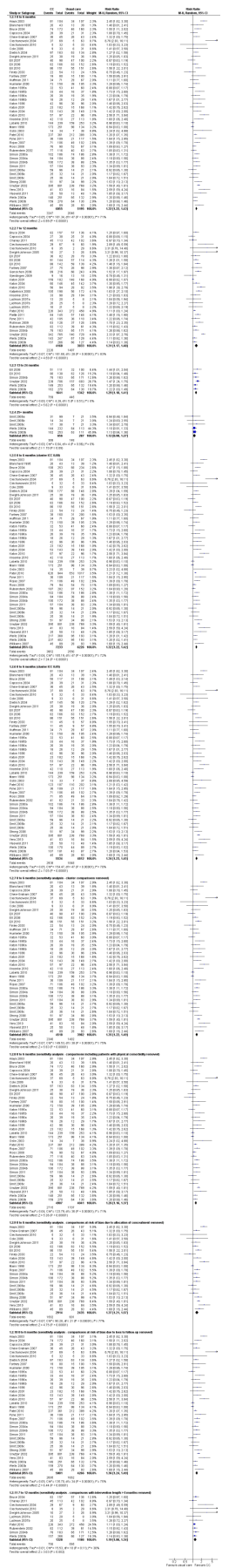




\section{Medium-term: 7 to 12 months}

Thirteen comparisons (4092 participants) reported medium-term continuous outcomes for depression for collaborative care versus usual care. Collaborative care was significantly more effective than usual care (SMD - $0.28,95 \%$ CI - 0.41 to $-0.15, \mathrm{I}^{2}=72 \%$ ) (Analysis $1.1)$.

Twenty-nine comparisons (8001 participants) reported mediumterm dichotomous outcomes for depression for collaborative care versus usual care. Collaborative care was significantly more effective than usual care (RR $1.31,95 \%$ CI 1.17 to $1.48, \mathrm{I}^{2}=83 \%$ ) (Analysis 1.2).

\section{Long-term: 13 to 24 months}

One comparison (1379 participants) reported long-term continuous outcomes for depression for collaborative care versus usual care. Collaborative care was significantly more effective than usual care (SMD - $0.35,95 \%$ CI -0.46 to -0.24 , I ${ }^{2}$ not applicable) (Analysis 1.1).

Six comparisons (2983 participants) reported long-term dichotomous outcomes for depression for collaborative care versus usual care. Collaborative care was significantly more effective than usual care (RR $1.29,95 \%$ CI 1.18 to $1.41, \mathrm{I}^{2}=0 \%$ ) (Analysis 1.2 ).

\section{Very long-term: 25 months or more}

No comparisons reported very long-term continuous outcomes for depression for collaborative care versus usual care.

Five comparisons (943 participants) reported very long-term dichotomous outcomes for depression for collaborative care versus usual care. There were no significant differences between the two groups (RR 1.12, 95\% CI 0.98 to $1.27, \mathrm{I}^{2}=0 \%$ ) (Analysis 1.2).

\section{I.3 Antidepressant medication use}

\section{Short-term: 0 to 6 months}

Forty-four comparison studies (10,117 participants) reported short-term dichotomous outcomes for antidepressant medication use. Collaborative care was significantly more effective than usual care (RR 1.47, 95\% CI 1.33 to $1.63, \mathrm{I}^{2}=81 \%$ ) (Analysis 1.3 ).

\section{Medium-term: 7 to 12 months}

Twenty-six comparisons (6486 participants) reported mediumterm dichotomous outcomes for antidepressant medication use. Collaborative care was significantly more effective than usual care (RR 1.43, 95\% CI 1.26 to $1.61, \mathrm{I}^{2}=78 \%$ ) (Analysis 1.3).

\section{Long-term: 13 to 24 months}

Six comparisons (2963 participants) reported long-term dichotomous outcomes for antidepressant medication use. Collaborative care was significantly more effective than usual care (RR 1.22, $95 \%$ CI 1.03 to $1.45, \mathrm{I}^{2}=54 \%$ ) (Analysis 1.3 ).

\section{Very long-term: 25 months or more}

Three comparisons (232 participants) reported very long-term dichotomous outcomes for antidepressant medication use. There were no significant differences between the two groups (RR 1.02, 95\% CI 0.87 to $1.21, \mathrm{I}^{2}=0 \%$ ) (Analysis 1.3 ).

\section{I.4 and I.5 Anxiety}

\section{Short-term: 0 to 6 months}

One comparison (876 participants) reported short-term continuous outcomes for anxiety for collaborative care versus usual care. Collaborative care was significantly more effective than usual care (SMD $-0.30,95 \%$ CI -0.44 to $-0.17, \mathrm{I}^{2}$ not applicable) (Analysis 1.4).

Four comparisons (1248 participants) reported short-term dichotomous outcomes for anxiety for collaborative care versus usual care. Collaborative care was significantly more effective than usual care (RR $1.50,95 \%$ CI 1.21 to $1.87, \mathrm{I}^{2}=55 \%$ ) (Analysis 1.5 ).

\section{Medium-term: 7 to 12 months}

One comparison (813 participants) reported medium- term continuous outcomes for anxiety for collaborative care versus usual care. Collaborative care was significantly more effective than usual care (SMD -0.33, 95\% CI -0.47 to -0.19, I² not applicable) (Analysis 1.4).

Five comparisons (1374 participants) reported medium- term dichotomous outcomes for anxiety for collaborative care versus usual care. Collaborative care was significantly more effective than usual care (RR $1.41,95 \%$ CI 1.18 to $1.69, \mathrm{I}^{2}=58 \%$ ) (Analysis 1.5 ).

\section{Long-term: 13 to 24 months}

One comparison (804 participants) reported long-term continuous outcomes for anxiety for collaborative care versus usual care. Collaborative care was significantly more effective than usual care (SMD $-0.20,95 \%$ CI -0.34 to $-0.06, \mathrm{I}^{2}$ not applicable) (Analysis 1.4).

One comparison (804 participants) reported long-term dichotomous outcomes for anxiety for collaborative care versus usual care. Collaborative care was significantly more effective than usual care (RR $1.26,95 \%$ CI 1.11 to 1.42 , $\mathrm{I}^{2}$ not applicable) (Analysis 1.5 ).

\section{Very long-term: 25 months or more}

No comparisons reported very long-term continuous or dichotomous outcomes for anxiety for collaborative care versus usual care.

\section{I.6 Anxiety medication use}

\section{Short-term: 0 to 6 months}

Three comparisons (1144 participants) reported short-term dichotomous outcomes for anxiety medication use. There were no 
significant differences between the two groups (RR 1.24, 95\% CI 0.93 to $1.63, \mathrm{I}^{2}=56 \%$ ) (Analysis 1.6 ).

\section{Medium-term: 7 to 12 months}

Four comparisons (1225 participants) reported medium-term dichotomous outcomes for anxiety medication use. Collaborative care was significantly more effective than usual care (RR 1.17, $95 \%$ CI 1.03 to $1.32, \mathrm{I}^{2}=0 \%$ ) (Analysis 1.6).

\section{Long-term: 13 to 24 months}

One comparison (804 participants) reported longer-term dichotomous outcomes for anxiety medication use. There were no significant differences between the two groups (RR 1.09, 95\% CI 0.92 to $1.30, \mathrm{I}^{2}$ not applicable) (Analysis 1.6).

\section{Very long-term: 25 months or more}

No comparisons reported very long-term dichotomous outcomes for anxiety medication use.

\section{I.7 Mental health quality of life}

\section{Short-term: 0 to 6 months}

Fourteen comparisons (4954 participants) reported short-term continuous outcomes for mental health quality of life. Collaborative care was significantly more effective than usual care (SMD $0.26,95 \%$ CI 0.13 to $0.38, \mathrm{I}^{2}=76 \%$ ) (Analysis 1.7 ).

\section{Medium-term: 7 to 12 months}

Eleven comparisons (3534 participants) reported medium-term continuous outcomes for mental health quality of life. Collaborative care was significantly more effective than usual care (SMD $0.20,95 \%$ CI 0.09 to $0.31, \mathrm{I}^{2}=58 \%$ ) (Analysis 1.7 ).

\section{Long-term: 13 to 24 months}

Three comparisons (1278 participants) reported long-term continuous outcomes for mental health quality of life. Collaborative care was significantly more effective than usual care (SMD 0.25, $95 \%$ CI 0.08 to $0.43, \mathrm{I}^{2}=51 \%$ ) (Analysis 1.7 ).

\section{Very long-term: 25 months or more}

Two comparisons (991 participants) reported very long-term continuous outcomes for mental health quality of life. There were no significant differences between the two groups (SMD 0.10, 95\% CI -0.03 to $0.23, \mathrm{I}^{2}=0 \%$ ) (Analysis 1.7 ).

\section{I.8 Physical health quality of life}

\section{Short-term: 0 to 6 months}

Ten comparisons (2957 participants) reported short-term continuous outcomes for physical health quality of life. There were no significant differences between the two groups (SMD 0.06, 95\% CI -0.01 to $0.13, \mathrm{I}^{2}=0 \%$ ) (Analysis 1.8 ).

\section{Medium-term: 7 to 12 months}

Ten comparisons (4552 participants) reported medium-term continuous outcomes for physical health quality of life. There were no significant differences between the two groups (SMD 0.07, 95\% CI -0.04 to $0.18, \mathrm{I}^{2}=67 \%$ ) (Analysis 1.8 ).

\section{Long-term: 13 to 24 months}

Four comparisons (2657 participants) reported long-term continuous outcomes for physical health quality of life. Collaborative care was significantly more effective than usual care (SMD 0.10 , $95 \%$ CI 0.02 to $0.17, \mathrm{I}^{2}=0 \%$ ) (Analysis 1.8 ).

\section{Very long-term: 25 months or more}

No comparisons reported very long-term continuous outcomes for physical health quality of life.

\section{9 and I.I0 Patient satisfaction}

Ten comparisons (3333 participants) reported continuous outcomes for patient satisfaction. Collaborative care was significantly more effective than usual care (SMD 0.31, 95\% CI 0.13 to 0.49 , $\mathrm{I}^{2}=82 \%$ ) (Analysis 1.9).

Twenty-four comparisons (5500 participants) reported dichotomous outcomes for patient satisfaction. Collaborative care was significantly more effective than usual care (RR 1.27, 95\% CI 1.18 to $1.38, \mathrm{I}^{2}=75 \%$ ) (Analysis 1.10 ).

\section{Collaborative care versus usual care (adolescents)}

\section{I and 2.2 Depression}

\section{Short-term: 0 to 6 months}

Two comparisons (471 participants) reported short-term continuous depression outcomes for collaborative care versus usual care in adolescents. There were no significant differences between the two groups (SMD - $0.17,95 \%$ CI -0.35 to $0.01, \mathrm{I}^{2}=0 \%$ ) (Analysis 2.1)

Two comparisons (460 participants) reported short-term dichotomous outcomes for depression for collaborative care versus usual care in adolescents. Collaborative care was significantly more effective than usual care (RR $0.73,95 \%$ CI 0.56 to $0.96, \mathrm{I}^{2}=0 \%$ ) (Analysis 2.2).

\section{Medium-term: 7 to 12 months}

One comparison (114 participants) reported medium-term continuous depression outcomes for collaborative care versus usual care in adolescents. There were no significant differences between 
the two (SMD $-0.32,95 \%$ CI -0.69 to $0.05, \mathrm{I}^{2}$ not applicable) (Analysis 2.1).

Two comparisons (441 participants) reported medium-term dichotomous outcomes for depression for collaborative care versus usual care in adolescents. There were no significant differences between the two groups (RR $1.05,95 \%$ CI 0.54 to $2.06, \mathrm{I}^{2}=32 \%$ ) (Analysis 2.2).

\section{Long-term: 13 to 24 months}

No comparisons reported long-term continuous outcomes for depression for collaborative care versus usual care in adolescents.

One comparison (322 participants) reported long-term dichotomous outcomes for depression for collaborative care versus usual care in adolescents. There were no significant differences between the two groups (RR $0.75,95 \%$ CI 0.51 to 1.11 , I² not applicable) (Analysis 2.2).

\section{Very long-term: 25 months or more}

No comparisons reported very long-term continuous or dichotomous outcomes for depression for collaborative care versus usual care in adolescents.

\subsection{Antidepressant medication use}

\section{Short-term: 0 to 6 months}

One comparison (335 participants) reported short-term dichotomous outcomes for antidepressant medication use. There were no significant differences between the two groups (RR 0.80, 95\% CI 0.47 to $1.35, \mathrm{I}^{2}$ not applicable) (Analysis 2.3).

\section{Medium-term: 7 to 12 months}

One comparison (327 participants) reported medium-term dichotomous outcomes for antidepressant medication use. There were no significant differences between the two groups (RR 0.80, 95\% CI 0.47 to $1.39, \mathrm{I}^{2}$ not applicable) (Analysis 2.3).

\section{Long-term: 13 to 24 months}

One comparison (321 participants) reported longer-term dichotomous outcomes for antidepressant medication use. There were no significant differences between the two groups (RR 0.68, 95\% CI 0.36 to $1.30, \mathrm{I}^{2}$ not applicable) (Analysis 2.3 ).

\section{Very long-term: 25 months or more}

No comparisons reported very long-term dichotomous outcomes for antidepressant medication use.

\subsection{Mental health quality of life}

\section{Short-term: 0 to 6 months}

Two comparisons (471 participants) reported short-term continuous outcomes for mental health quality of life. There were no significant differences between the two groups (SMD 0.15, 95\% CI -0.03 to $0.33, \mathrm{I}^{2}=0 \%$ ) (Analysis 2.4 ).

\section{Medium-term: 7 to 12 months}

Two comparisons (441 participants) reported medium-term continuous outcomes for mental health quality of life. There were no significant differences between the two groups (SMD 0.05, 95\% CI -0.24 to $0.33, \mathrm{I}^{2}=47 \%$ ) (Analysis 2.4 ).

\section{Long-term: 13 to 24 months}

One comparison (322 participants) reported medium-term continuous outcomes for mental health quality of life. There were no significant differences between the two groups (SMD 0.09, 95\% CI -0.13 to 0.31 , I ${ }^{2}$ not applicable) (Analysis 2.4).

\section{Very long-term: 25 months or more}

No comparisons reported very long-term continuous outcomes for mental health quality of life.

\subsection{Physical health quality of life}

\section{Short-term 0 to 6 months}

One comparison (127 participants) reported short-term continuous outcomes for physical health quality of life. There were no significant differences between the two groups (SMD - 0.25, 95\% CI -0.59 to $0.10, \mathrm{I}^{2}$ not applicable) (Analysis 2.5).

\section{Medium-term: 7 to 12 months}

Two comparisons (114 participants) reported medium-term continuous outcomes for physical health quality of life. There were no significant differences between the two groups (SMD 0.12, 95\% CI -0.25 to $0.49, \mathrm{I}^{2}$ not applicable) (Analysis 2.5 ).

\section{Long-term: 13 to 24 months}

No comparisons reported long-term continuous outcomes for physical health quality of life.

\section{Very long-term: 25 months or more}

No comparisons reported very long-term continuous outcomes for physical health quality of life.

\subsection{Patient satisfaction}

Two comparisons (471 participants) reported continuous outcomes for patient satisfaction. There were no significant differences between the two groups (SMD 0.09, 95\% CI -0.38 to 0.57, $\mathrm{I}^{2}=82 \%$ ) (Analysis 2.6).

No comparisons reported dichotomous outcomes for patient satisfaction. 


\section{Collaborative care versus feedback (adults)}

\section{I Depression}

\section{Short-term: 0 to 6 months}

No comparisons reported continuous outcomes for depression for collaborative care versus feedback.

One comparison (396 participants) reported dichotomous outcomes for depression for collaborative care versus feedback. Collaborative care was significantly more effective than feedback (RR $1.25,95 \%$ C I 1.02 to 1.53 , I² not applicable) (Analysis 3.1).

\section{Medium-term: 7 to 12 months}

No comparisons reported medium-term continuous outcomes for depression for collaborative care versus feedback.

No comparisons reported medium-term dichotomous outcomes for depression for collaborative care versus feedback.

\section{Long-term: 13 to 24 months}

No comparisons reported long-term continuous outcomes for depression for collaborative care versus feedback.

No comparisons reported long-term dichotomous outcomes for depression for collaborative care versus feedback.

\section{Very long-term: 25 months or more}

No comparisons reported very long-term continuous outcomes for depression for collaborative care versus feedback.

No comparisons reported very long-term dichotomous outcomes for depression for collaborative care versus feedback.

\section{Collaborative care versus consultation-liaison (adults)}

\section{I Depression}

\section{Short-term: 0 to 6 months}

No comparisons reported continuous outcomes for depression for collaborative care versus consultation-liaison.

One comparison (77 participants) reported short-term dichotomous outcomes for depression for collaborative care versus consultation-liaison. There were no significant differences between the two groups (RR $1.30,95 \%$ CI 0.43 to 3.89 , I² not applicable) (Analysis 4.1).

\section{Medium-term: 7 to 12 months}

One comparison (77 participants) reported medium-term dichotomous outcomes for depression for collaborative care versus consultation-liaison. There were no significant differences between the two groups (RR $1.14,95 \%$ CI 0.40 to 3.22 , I ${ }^{2}$ not applicable) (Analysis 4.1).

\section{Long-term: 13 to 24 months}

No comparisons reported long-term continuous or dichotomous outcomes for depression for collaborative care versus consultationliaison.

\section{Very long-term: 25 months or more}

No comparisons reported very long-term continuous or dichotomous outcomes for depression for collaborative care versus consultation-liaison.

\section{Collaborative care plus consultation-liaison versus collaborative care (adults)}

\section{I Depression}

\section{Short-term: 0 to 6 months}

One comparison (128 participants) reported short-term dichotomous outcomes for depression for collaborative care plus consultation-liaison versus collaborative care. Collaborative care plus consultation-liaison was significantly more effective than usual care (RR $1.27,95 \%$ CI 1.00 to $1.62, \mathrm{I}^{2}$ not applicable) (Analysis 5.1 ).

\section{Medium-term: 7 to 12 months}

No comparisons reported medium-term dichotomous outcomes for depression for collaborative care plus consultation-liaison versus collaborative care.

\section{Long-term: 13 to 24 months}

No comparisons reported long-term dichotomous outcomes for depression for collaborative care plus consultation-liaison versus collaborative care.

\section{Very long-term: 25 months or more}

One comparison (133 participants) reported very long-term dichotomous outcomes for depression for collaborative care plus consultation-liaison versus collaborative care. There were no significant differences between the two groups (RR 1.31, 95\% CI 0.80 to $2.16, \mathrm{I}^{2}$ not applicable) (Analysis 5.1).

\section{Collaborative care versus enhanced referral (adults)}

\section{I Depression}

\section{Short-term: 0 to 6 months}

One comparison (1220 participants) reported continuous outcomes for depression for collaborative care versus enhanced referral. There were no significant differences between the two groups 
(SMD $0.08,95 \%$ CI -0.03 to 0.19 , $\mathrm{I}^{2}$ not applicable) (Analysis 6.1).

No studies reported dichotomous outcomes for depression for collaborative care versus enhanced referral.

\section{Medium-term: 7 to 12 months}

No comparisons reported medium-term continuous outcomes for depression for collaborative care versus enhanced referral.

\section{Long-term: 13 to 24 months}

No comparisons reported long-term continuous outcomes for depression for collaborative care versus enhanced referral.

\section{Very long-term: 25 months or more}

No comparisons reported very long-term continuous outcomes for depression for collaborative care versus enhanced referral.

\section{Collaborative care (psychotherapy) versus collaborative care (medication) (adults)}

\section{I Depression}

\section{Short-term: 0 to 6 months}

No comparisons reported continuous outcomes for depression for collaborative care (psychotherapy) versus collaborative care (medication).

One comparison (521 participants) reported short-term dichotomous outcomes for depression for collaborative care (psychotherapy) versus collaborative care (medication). There were no significant differences between the two groups (RR 1.00, 95\% CI 0.87 to $1.15, \mathrm{I}^{2}$ not applicable) (Analysis 7.1).

\section{Medium-term: 7 to 12 months}

No comparisons reported continuous outcomes for depression for collaborative care (psychotherapy) versus collaborative-care (medication).

One comparison (513 participants) reported medium-term dichotomous outcomes for depression for collaborative care (psychotherapy) versus collaborative care (medication). There were no significant differences between the two groups (RR 1.02, 95\% CI 0.88 to $1.18, \mathrm{I}^{2}$ not applicable) (Analysis 7.1 ).

\section{Long-term: 13 to 24 months}

No comparisons reported continuous outcomes for depression for collaborative care (psychotherapy) versus collaborative care (medication).

One comparison (523 participants) reported long-term dichotomous outcomes for depression for collaborative care (psychotherapy) versus collaborative care (medication). There were no significant differences between the two groups (RR 1.02, 95\% CI 0.88 to $1.17, \mathrm{I}^{2}$ not applicable) (Analysis 7.1).

\section{Very long-term: 25 months or more}

No comparisons reported continuous outcomes for depression for collaborative care (psychotherapy) versus collaborative care (medication).

One comparison (485 participants) reported very long-term dichotomous outcomes for depression for collaborative care (psychotherapy) versus collaborative care (medication). There were no significant differences between the two groups (RR 1.03, 95\% CI 0.90 to $1.18, \mathrm{I}^{2}$ not applicable) (Analysis 7.1 ).

\section{Collaborative care plus psychotherapy versus collaborative care (adults)}

\section{I and 8.2 Depression}

\section{Short-term: 0 to 6 months}

One comparison (43 participants) reported continuous outcomes for depression for collaborative care plus psychotherapy versus collaborative care. There were no significant differences between the two groups (SMD $-0.27,95 \% \mathrm{CI}-0.87$ to 0.33 , I $\mathrm{I}^{2}$ not applicable) (Analysis 8.1).

Two comparisons (488 participants) reported short-term dichotomous outcomes for depression for collaborative care plus psychotherapy versus collaborative care. There were no significant differences between the two groups (RR 1.14, 95\% CI 0.97 to $1.33, \mathrm{I}^{2}=0 \%$ ) (Analysis 8.2).

\section{Medium-term: 7 to 12 months}

No comparisons reported medium-term continuous outcomes for depression for collaborative care plus psychotherapy versus collaborative care.

One comparison (41 participants) reported medium-term dichotomous outcomes for depression for collaborative care plus psychotherapy versus collaborative care. There were no significant differences between the two groups (RR 1.17, 95\% CI 0.79 to $1.75, \mathrm{I}^{2}$ not applicable) (Analysis 8.2).

\section{Long-term: 13 to 24 months}

No comparisons reported long-term continuous outcomes for depression for collaborative care plus psychotherapy versus collaborative care.

No comparisons reported long-term dichotomous outcomes for depression for collaborative care plus psychotherapy versus collaborative care.

\section{Very long-term: 25 months or more}

No comparisons reported very long-term continuous outcomes for depression for collaborative care plus psychotherapy versus collaborative care.

One comparison (137 participants) reported very long-term dichotomous outcomes for depression for collaborative care plus 
psychotherapy versus collaborative care. There were no significant differences between the two groups (RR 1.43, 95\% CI 0.90 to 2.26, $\mathrm{I}^{2}$ not applicable) (Analysis 8.2).

\section{Sensitivity analyses}

The main analysis of the effects of collaborative care on continuous depression outcomes at six months (SMD - $0.34,95 \%$ CI -0.41 to -0.27) was not markedly changed when the intracluster correlation coefficient (ICC) used to analyse cluster comparisons was 0.00 (SMD - $0.33,95 \%$ CI -0.39 to -0.26) or 0.05 (SMD -0.34, 95\% CI -0.41 to -0.26) (Analysis 1.1).

The main analysis of the effects of collaborative care on continuous depression outcomes at six months (SMD -0.34, 95\% CI 0.41 to -0.27 ) was not markedly changed when sensitivity analysis removed cluster comparisons (SMD -0.37, 95\% CI - 0.46 to 0.28 ), comparisons with inclusion criteria of physical comorbidity (SMD - $0.29,95 \%$ CI -0.37 to -0.21 ) or comparisons at unclear or high risk of bias in terms of allocation concealment (SMD $0.34,95 \%$ CI -0.42 to -0.26 ) or loss to follow-up (SMD -0.33, 95\% CI -0.40 to -0.26 ) (Analysis 1.1 ).

The effects of collaborative care on continuous depression outcomes at 12 months (SMD - $0.28,95 \%$ CI -0.41 to -0.15) changed to SMD -0.19 (95\% CI -0.30 to -0.08$)$ when comparisons including intervention beyond six months were removed.

The main analysis of the effects of collaborative care on dichotomous depression outcomes at six months (RR 1.32, 95\% CI 1.22 to 1.43 ) was not markedly changed when the estimates of the ICC used to analyse cluster comparisons were 0.00 (RR 1.32, 95\% CI 1.22 to 1.42 ) or 0.05 (RR $1.34,95 \%$ CI 1.23 to 1.45 ) (Analysis $1.2)$.

The main analysis of the effects of collaborative care on dichotomous depression outcomes at six months (RR 1.32, 95\% CI 1.22 to 1.43 ) was not markedly changed when sensitivity analysis removed cluster comparisons (RR 1.35, 95\% CI 1.22 to 1.49), comparisons with inclusion criteria of physical comorbidity (RR 1.26, 95\% CI 1.16 to 1.37 ) and comparisons at unclear or high risk of bias in allocation concealment (RR $1.37,95 \% \mathrm{CI} 1.21$ to 1.57 ) or loss to follow-up (RR 1.36, 95\% CI 1.24 to 1.49) (Analysis 1.2). The effects of collaborative care at 12 months (RR 1.31, 95\% CI 1.17 to 1.48 ) changed to RR 1.19 (95\% CI 1.06 to 1.34 ) when comparisons including intervention beyond six months were removed.

\section{DISCUSSION}

We have summarised a large body of evidence from 79 randomised controlled trials (RCTs) (90 comparisons) which predominantly compare collaborative care with usual care, although there are a small number of comparisons of types of collaborative care, or comparisons of collaborative care and other active interventions. This is the first Cochrane review of this body of evidence and our main findings are outlined below.

\section{Summary of main results}

\section{Collaborative care versus usual care (adults)}

In terms of primary outcomes, collaborative care for patients with depression is more effective than usual care in terms of depression outcomes at around six months, 12 months, and 24 months, although the effects were not significant after 24 months. Collaborative care for patients with anxiety is more effective than usual care in terms of anxiety outcomes at around six months, 12 months and 24 months.

In terms of secondary outcomes, collaborative care for patients with depression increases rates of antidepressant use at around six months, 12 months and 24 months, although the effects are not significant beyond 24 months. Collaborative care for patients with anxiety led to significantly higher rates of anxiety medication use at 12 months.

Collaborative care is more effective than usual care in terms of mental health quality of life at around six months, 12 months and 24 months. Collaborative care is more effective than usual care in terms of physical health quality of life at around 24 months only. Collaborative care is more effective than usual care in terms of patient satisfaction post-intervention.

\section{Other comparisons}

Collaborative care was not significantly more effective than usual care in adolescents with depression at around six months or 12 months when measured using continuous outcomes, although the intervention was significantly more effective than usual care at six months when measured using dichotomous outcomes. Collaborative care for adolescents with depression had no significant effects on antidepressant use. There were no significant differences in mental or physical health quality of life or patient satisfaction. There were a limited number of randomised comparisons of collaborative care versus other interventions. Collaborative care was significantly more effective than feedback alone, but no more effective than consultation-liaison or enhanced referral. There was limited evidence that adding consultation-liaison to collaborative care was significantly more effective than collaborative care alone at around six months only. There were no significant differences between collaborative care and psychotherapy compared with collaborative care alone. There were no differences between collaborative care based on a psychotherapy model, and collaborative care based on a medication model. 


\section{Overall completeness and applicability of evidence}

This review has included 79 RCTs (90 comparisons) of collaborative care most of which focus on improving mental health outcomes for adults with depression in primary care. This means that collaborative care for anxiety and depression is one of the most well evaluated interventions in mental health in primary care.

\section{Types of study design}

Most studies were individually randomised trials but a proportion (21; 23\%) used cluster randomisation. Cluster-RCTs are recommended for testing systems-level interventions such as collaborative care (Ukoumunne 1999), as patient randomised trials may be vulnerable to contamination i.e. changes in the behaviour of primary care providers influenced by system-level changes such as advice from mental health specialists and routine screening and feedback of patients' mental health status (Richards 2008a). However, cluster-RCTs generally require larger patient samples and may be vulnerable to other sources of bias (selective patient recruitment after cluster randomisation; baseline imbalance due to the smaller number of clusters recruited; loss of clusters; and incorrect analysis). This review explored the inclusion of cluster-RCTs in sensitivity analysis and there was no evidence that the main outcomes were sensitive to the inclusion of such trials, or the estimates of the level of clustering used to estimate outcomes in the meta-analysis.

\section{Types of participants}

Although the majority of the included studies have been conducted in the US, more studies are being conducted worldwide, and the positive outcomes reported in the US do seem to be replicated in other countries in Europe (Chew-Graham 2007; Gensichen 2009; Richards 2012) and wider contexts (Araya 2003; Patel 2010; Rojas 2007). However, given the more limited evidence base, the main findings of the review need to be interpreted with more caution when considering other settings.

Originally, collaborative care studies were conducted on patients with depression. However, more recently the study of collaborative care has diversified, recruiting participants with anxiety disorders, and patients with diagnosed physical health conditions (e.g. specialist centres treating people with lung cancer or diabetes).

Clinical diagnosis was not necessarily a prerequisite for inclusion in the studies and therefore we included a wide range of symptoms and/or disorders (subthreshold, mild and major depression, chronic, postnatal). Studies that use diagnostic criteria to screen participants for eligibility are often prioritised over studies that use self-report outcome measures or clinician judgement, particularly as evidence based guidelines often exclude the latter from their reviews of the literature (NICE 2010). Whilst positive outcomes may be more likely when interventions are targeted to a specific diagnostic group (Roth 1996), studies where interventions are offered based on levels of symptoms rather than research diagnoses may be more representative of routine practice.

\section{Types of intervention}

Collaborative care is a complex intervention which is difficult to define precisely. This review based inclusion and exclusion criteria on a published definition of collaborative care (Gunn 2006). Whilst this was considered the most comprehensive and internally consistent definition to date, there was still variation in what was delivered as part of a collaborative care model in relation to all four intervention criteria.

In terms of a multi-professional approach some studies included just two health professionals (primary care provider and case manager/and or mental health specialist), while others included a primary care provider, case manager and a mental health specialist. There was variation in the amount of structure in the management plan, where some studies were highly prescriptive (e.g. providing a written manual for the primary care provider and/or the case manager to follow) and others were less so (providing written treatment guidelines and encouraging individualised treatment plans). There was variation also in the intensity of the intervention in each study in terms of number of follow-ups scheduled (ranging from 1 to 20+); method of delivery (face-to-face, telephone or a combination); and session duration. This variation in 'key ingredients' of collaborative care complicates the interpretation of the results.

We excluded studies from this review examining stepped care models where access to collaborative care was restricted and reserved for a small proportion of participants meeting specific criteria, as it would have been impossible to assess the added value of the collaborative care element. This may be an important consideration, particularly in the UK where stepped care is the recommended service model for depression and anxiety (NICE 2010).

\section{Types of comparison}

Most of the studies compared collaborative care with 'usual care'. A limitation of this review is that 'usual care' is hard to define and included studies did not clearly describe the key elements. Many of the most traditional 'usual care' studies also included some limited level of intervention (distribution of treatment guidelines, informing patients of depression status, training and education of primary care practitioners). The evidence for these interventions delivered in isolation is limited (Bower 2005) but such interventions could result in a lower treatment effect.

Several studies have compared collaborative care with another active treatment (such as consultation-liaison or enhanced referral) but the numbers of available comparisons is low and confidence in the conclusions about their relative value is limited. 


\section{Quality of the evidence}

Most of the 90 comparisons were included in analyses of depression outcomes, enabling estimates of the effects of collaborative care in the short-term and medium-term with a high level of precision.

We found clinical and methodological heterogeneity in terms of participants, interventions, comparisons and outcome measures. In primary analyses, the value of the $\mathrm{I}^{2}$ statistic for the continuous measure of depression outcome at six months was 34\%; 'moderate' according to recommended criteria (Deeks 2011). For dichotomous measures of depression outcome at six months it was $71 \%$ indicating 'substantial' heterogeneity according to recommended criteria. Using the same criteria, 'considerable' heterogeneity was apparent in the analysis of antidepressant use at six months ( ${ }^{2}$ statistic $=81 \%)$. The $95 \%$ confidence intervals around the $\mathrm{I}^{2}$ estimate, calculated using the Stata command heterogi, are presented in Appendix 2. We used random-effects models in all analyses. We did not identify any adverse outcomes. Trials in this area of research rarely record adverse events.

Applying'Risk of bias' criteria to the studies has identified methodological limitations in the studies, although some of these (e.g. blinding of participants and clinicians) reflect the reality of conducting complex intervention trials in practice. Some studies rated as 'high risk' of bias for 'blinding of participants' used self-reported outcomes which may not be as vulnerable to bias as an unblinded external observer. There was no evidence that removing studies at high risk of bias (assessed in terms of allocation concealment) had a large effect on the estimate of treatment effect in the main analyses. Studies varied in whether they reported outcomes in terms of continuous measures or dichotomous outcomes, and there is a risk of bias if this represents selective reporting. However, this is very difficult to judge without access to study protocols.

\section{Potential biases in the review process}

Since the published protocol, we have made several changes in response to peer review, and as a result of internal discussions, on the best way to synthesise data about a complex and multifaceted intervention. These changes are documented in line with good practice.

We did not contact all authors to collect missing data. Given the size and complexity of the review this would have required multiple requests for data from many authors and study timelines did not allow for this task.

There are a number of analyses that we had planned, but given the size and complexity of the review we have been unable to complete such analyses because of time constraints.

We were unable to conduct a rigorous and reliable analysis of social function outcomes in the time frame of the review, as the measures reported were highly varied and their comparability difficult to judge without extensive work on individual scales. We will add these outcomes at a later date.

The protocol discussed several subgroup analyses and exploratory meta-regression. We analysed outcomes separately for adolescents and adults, and for interventions targeting patients with depression and anxiety. We did not conduct subgroup analyses for country, location of recruitment, ethnicity, or aspects of the intervention, or conduct the exploratory meta-regression. Exploration of the impact of such factors will benefit from a multivariate approach which can assess the relative importance of factors, rather than a series of single subgroup analyses which may be confounded with other important factors. Such a meta-regression analysis will require extensive imputation of missing outcome data, and translation of continuous and dichotomous outcomes into a common format. These activities could not be completed in the time frame of the review, but we will add these at a later date.

We did not extract data from studies that reported antidepressant medication as a continuous outcome only, or from studies that reported quality of life as a dichotomous outcome only, or as a general quality of life measure only that combined physical and mental health. In all cases this represented less than $5 \%$ of studies. We extracted data on satisfaction at six months only. Although it is possible that satisfaction could change over time, our judgement was that satisfaction measures are fundamentally associated with views of the treatment process, and thus measures close to treatment receipt are much more likely to be accurate and unconfounded by memory issues.

We have included analyses of collaborative care plus enhancement versus collaborative care. We accept that such studies provide an assessment of the effects of the enhancement, not collaborative care. However, we felt that such analyses were of relevance, as it is an important clinical issue as to whether the effects of collaborative care can be increased by adding other features to the basic model. Our analysis split outcomes into four time periods based on time since randomisation. The nature of collaborative care means that interventions are sometimes provided over longer periods of time, and thus it is possible that long-term effects of collaborative care (i.e. those in the 12-month period and beyond) do not reflect any enduring effect of the intervention, but simply reflect those interventions that are extended beyond the initial outcome period (0 to 6 months). We conducted a sensitivity analysis, removing studies where the intervention extended beyond six months, to assess whether the effects found at the 7- to 12-month time point were significantly different when studies with longer-term interventions were excluded. In both outcomes, the effect of collaborative care at 7 to 12 months was reduced, which supports the suggestion that long-term effects on outcome are more likely when the intervention is also conducted over the longer-term. However, the effects of collaborative care are still statistically significant at both times points even with longer-term interventions removed, and this issue needs further research. 
We did not contact all first authors or experts in the field to check for additional studies to those found through our searches.

\section{Agreements and disagreements with other studies or reviews}

A number of reviews have examined the effectiveness of collaborative care, enhanced care, disease management and complex system interventions (Badamgarav 2003; Bijl 2004; Gensichen 2009; Gilbody 2006; Gunn 2006; Kates 2007; Neumeyer-Gromen 2004). These reviews have used a mixture of narrative and metaanalyses to examine outcomes including: depression symptoms and caseness, patient satisfaction, adherence to treatment and costeffectiveness.

These reviews mainly include RCTs. A previous review conducted in 2006 included 37 RCTs of collaborative care (Gilbody 2006) using broader inclusion criteria for collaborative care (Katon 2001). A recent review (Thota 2012) identified a further 32 studies between 2004 and 2009. The current review demonstrates the increase in activity in the implementation and evaluation of collaborative care, although some of the differences in the numbers of studies included in different reviews represent differences in exact inclusion and exclusion criteria.

Like the current review, all of the published reviews concluded collaborative care was effective in the short-term and mediumterm, and some reviews also considered longer-term outcomes, reporting trends for significant effects up to five years (Gilbody 2006).

Less evidence has been reported for other outcomes such as quality of life and patient satisfaction although some previous reviews have reported potential positive outcomes for collaborative care in terms of patient satisfaction (Badamgarav 2003; NeumeyerGromen 2004; Thota 2012).

We did not identify any reviews that examined collaborative care for adults with anxiety; across different age ranges (including adolescents and older age) and compared with other active treatments (such as consultation-liaison and enhanced referral).

\section{AUTHORS'CONCLUSIONS}

\section{Implications for practice}

This review has demonstrated clear and robust evidence of effectiveness for collaborative care in improving depression outcomes in the short- and medium-term.

These findings have important implications for current clinical guidelines for depression. The overall finding that collaborative care is associated with improvements in depression is based on a large and varied database and is highly likely to be rigorous. However, debate will continue over the magnitude of the benefits. The standardised mean difference (SMD) demonstrated in the main analyses, although significant, is modest by current convention (Lipsey 1990), and less than some important comparison treatments (such as cognitive behaviour therapy (CBT)) when evaluated in contexts other than primary care (Churchill 2002; NICE 2010). Although there is a lack of consensus on 'minimally clinically important differences' in mental health, a SMD of 0.5 has been used previously as a criteria for adoption in the UK, and the data in this review shows effects which are less than this, and some benefits (such as those on physical health quality of life) are statistically significant but potentially of limited clinical significance.

However, the benefits of collaborative care are similar to other treatments (such as CBT and other psychological therapies) when delivered in primary care settings (Cape 2010) and it is important to note that the benefits, although modest, do seem to endure over time, possibly reflecting the chronic disease management basis of the collaborative care intervention.

Currently the National Institute for Clinical Excellence (NICE) in the UK only recommends collaborative care at step 3 for people with long-term physical conditions and depression (NICE 2009). This current review did not find that excluding studies in patients with long-term physical conditions made a substantive difference to the findings of the review (main analysis SMD -0.34; sensitivity analysis excluding studies in patients with comorbidity SMD -0.29). As noted previously, such simple comparisons are problematic, as there are many additional differences between studies that could account for this variation, beyond the types of patients recruited. For example, collaborative care is a complex intervention and there is significant variation in the exact nature of the intervention in the included studies, as well as differences in patient populations, contexts, comparisons, and design. Replication of an earlier meta-regression including the new studies is required (Bower 2006), accounting for the full range of studies and relevant characteristics, and we will conduct this as an update to this review.

\section{Implications for research}

The evidence for the effectiveness of collaborative care for depression in the short- and medium-term is robust. There is a need for further research in collaborative care for anxiety, in patients with depression and long-term physical health conditions, and in different age groups (adolescents and older age). Comparisons of collaborative care models with other interventions would also be useful to better determine its optimal place in current clinical pathways.

Exploration of the moderators and mediators of the effects of collaborative care (Kraemer 2002) might provide useful guidance on how current models could enhance effectiveness through greater focus on 'active ingredients' and better targeting of patient populations most likely to benefit. 
Improvements in the way research is reported are required. We were unable to make accurate judgements about many risk of bias issues, and we could not include large numbers of outcomes in the analyses because key data were missing. Researchers should also be encouraged to include more consistent data on the actual interventions included in collaborative care studies and report adverse events.

\section{ACKNOWLEDGEMENTS}

The reviewers would like to thank the editorial team of the Cochrane Collaboration Depression, Anxiety and Neurosis Group for its advice and support.
The review was supported by the National Institute for Health Research (NIHR) Cochrane Review Incentive Scheme 2012.

The reviewers would also like to thank: Jasmine Knopp, Chris Keyworth, Dr Charlotte Garrett, Dr Cassandra Kenning, Angee Khara, Jacqueline Hill, Dr Chris Gibbons and Sarah Tully for their support with the data extraction.

Thank you also to the study authors who have responded to queries and requests for further information.

Peter Coventry (PC) is funded by the NIHR Collaboration for Leadership in Applied Health Research and Care for Greater Manchester. Peter Bower (PB) acknowledges the support of the NIHR School for Primary Care Research. The views expressed in this article are those of the authors and not necessarily those of the NIHR.

\section{R E F E R N C E S}

\section{References to studies included in this review}

Adler 2004 \{published data only\}

* Adler DA, Bungay KM, Wilson IB, Pei Y, Supran S, Peckham E, et al.The impact of a pharmacist intervention on 6-month outcomes in depressed primary care patients. General Hospital Psychiatry 2004;26(3):199-209. Bungay KM, Adler DA, Rogers WH, McCoy C, Kaszuba $\mathrm{M}$, Supran S, et al.Description of a clinical pharmacist intervention administered to primary care patients with depression. General Hospital Psychiatry 2004;26(3):210-8.

Araya 2003 \{published data only\}

Araya R, Flynn T, Rojas G, Fritsch R, Simon G. Costeffectiveness of a primary care treatment program for depression in low-income women in Santiago, Chile. American Journal of Psychiatry 2006;163(8):1379-87.

* Araya R, Rojas G, Fritsch R, Gaete J, Rojas M, Simon G, et al.Treating depression in primary care in low-income women in Santiago, Chile: a randomised controlled trial. Lancet 2003;361(9362):995-1000.

Rojas G, Araya R, Simon G, Fritsch R, Gaete J. Treating depression among low income women in primary care, Santiago, Chile. 156th Annual Meeting of the American Psychiatric Association; 2003 May 17-22; San Francisco CA. 2003:NR109.

Asarnow 2005 \{published data only\}

Asarnow J, McKowen J, Jaycox L. Improving care for depression: integrating evidence-based depression treatment within primary care services. In: Essau $\mathrm{C}$ editor(s). Treatments for Adolescent Depression: Theory and Practice. Oxford: Oxford University Press, 2009:159-74.

* Asarnow JR, Jaycox LH, Duan N, LaBorde AP, Rea MM, Murray P, et al.Effectiveness of a quality improvement intervention for adolescent depression in primary care clinics: a randomized controlled trial. JAMA 2005;293(3): 311-9.

Asarnow JR, Jaycox LH, Tang L, Duan N, LaBorde AP, Zeledon LR, et al.Long-term benefits of short-term quality improvement interventions for depressed youths in primary care. American Journal of Psychiatry 2009;166(9):1002-10.

\section{Bartels 2004 \{published data only\}}

Arean PA, Ayalon L, Jin C, McCulloch CE, Linkins K, Chen $\mathrm{H}$, et al.Integrated specialty mental health care among older minorities improves access but not outcomes: Results of the PRISMe study. International Journal of Geriatric Psychiatry 2008;23(10):1086-92.

Azar AR, Chopra MP, Cho LY, Coakley E, Rudolph JL. Remission in major depression: results from a geriatric primary care population. International Journal of Geriatric Psychiatry 2011;26(1):48-55.

* Bartels SJ, Coakley EH, Zubritsky C, Ware JH, Miles $\mathrm{KM}$, Arean PA, et al.Improving access to geriatric mental health services: A randomized trial comparing treatment engagement with integrated versus enhanced referral care for depression, anxiety, and at-risk alcohol use. American Journal of Psychiatry 2004;161(8):1455-62.

Chen H, Coakley EH, Cheal K, Maxwell J, Costantino G, Krahn DD, et al.Satisfaction with mental health services in older primary care patients. American Journal of Geriatric Psychiatry 2006;14(4):371-9.

Datto CJ, Thompson R, Knott K, Katz IR. Older adult report of change in depressive symptoms as a treatment decision tool. Journal of the American Geriatrics Society 2006;54(4):627-31.

Gallo JJ, Zubritsky C, Maxwell J, Nazar M, Bogner HR, Quijano LM, et al.Primary care clinicians evaluate integrated and referral models of behavioral health care for older adults: results from a multisite effectiveness trial 
(PRISM-e). Annals of Family Medicine 2004;2(4):305-9. Krahn DD, Bartels SJ, Coakley E, Oslin DW, Chen H, McIntyre J, et al.PRISM-E: comparison of integrated care and enhanced specialty referral models in depression outcomes. Psychiatric Services 2006;57(7):946-53.

Levkoff SE, Chen H, Coakley E, Herr EC, Oslin DW, Katz I, et al.Design and sample characteristics of the PRISM-E multisite randomized trial to improve behavioral health care for the elderly. Journal of Aging and Health 2004;16(1): 3-27.

Mavandadi S, Ten Have TR, Katz IR, Durai UN, Krahn $\mathrm{DD}$, Llorente MD, et al.Effect of depression treatment on depressive symptoms in older adulthood: the moderating role of pain. Journal of the American Geriatrics Society 2007; 55(2):202-11.

Wiley-Exley E, Domino ME, Maxwell J, Levkoff SE. Costeffectiveness of integrated care for elderly depressed patients in the PRISM-E study. The Journal of Mental Health Policy and Economics 2009;12(4):205-13.

Wittink MN, Oslin D, Knott KA, Coyne JC, Gallo JJ, Zubritsky C. Personal characteristics and depression-related attitudes of older adults and participation in stages of implementation of a multi-site effectiveness trial (PRISME). International Journal of Geriatric Psychiatry 2005;20(10): 927-37.

Zanjani F, Mavandadi S, TenHave T, Katz I, Durai NB, Krahn D, et al.Longitudinal course of substance treatment benefits in older male veteran at-risk drinkers. Journals of Gerontology 2008;63(1):98-106.

Blanchard 1995 \{published data only\}

* Blanchard MR, Waterreus A, Mann AH. The effect of primary care nurse intervention upon older people screened as depressed. International Journal of Geriatric Psychiatry 1995;10(4):289-98.

Mann AH, Blanchard M, Waterreus A. Depression in older people. Some criteria for effective treatment. Encephale 1993;19(Spec No 3):445-50.

Waterreus A, Blanchard M, Mann AH. Community psychiatric nurses for the elderly: well tolerated, few sideeffects and effective in the treatment of depression. Journal of Clinical Nursing 1994;3(5):299-306.

Bogner 2008 \{published data only\}

* Bogner HR, de Vries HF. Integration of depression and hypertension treatment: a pilot, randomized controlled trial. Annals of Family Medicine 2008;6(4):295-301.

Bogner 2010 \{published data only\}

* Bogner HR, de Vries HF. Integrating type 2 diabetes mellitus and depression treatment among African Americans: a randomized controlled pilot trial. Diabetes Educator 2010;36(2):284-92.

Bruce 2004 \{published data only\}

Alexopoulos GS, Katz IR, Bruce ML, Heo M, Ten Have T, Raue P, et al.Remission in depressed geriatric primary care patients: a report from the PROSPECT study. American Journal of Psychiatry 2005;162(4):718-24.

Alexopoulos GS, Reynolds CF III, Bruce ML, Katz IR, Raue PJ, Mulsant $\mathrm{BH}$, et al.Reducing suicidal ideation and depression in older primary care patients: 24-month outcomes of the PROSPECT study. American Journal of Psychiatry 2009;166(8):845-8.

Bao Y, Alexopoulos GS, Casalino LP, Ten Have TR, Donohue JM, Post EP, et al.Collaborative depression care management and disparities in depression treatment and outcomes. Archives of General Psychiatry 2011;68(6): 627-36.

Bao Y, Casalino LP, Ettner SL, Bruce ML, Solberg LI, Unutzer J. Designing payment for collaborative care for depression in primary care. Health Services Research 2011; 46(5):1436-51.

Bao Y, Post EP, Ten TR, Schackman BR, Bruce ML. Achieving effective antidepressant pharmacotherapy in primary care: the role of depression care management in treating late-life depression. Journal of the American Geriatrics Society 2009;57(5):895-900.

Bogner HR, Bruce ML, Reynolds CF 3rd, Mulsant BH, Cary MS, Morales K, et al.The effects of memory, attention, and executive dysfunction on outcomes of depression in a primary care intervention trial: the PROSPECT study. International Journal of Geriatric Psychiatry 2007;22(9): 922-9.

Bogner HR, Cary MS, Bruce ML, Reynolds CF III, Mulsant $B$, Ten-Have $T$, et al.The role of medical comorbidity in outcome of major depression in primary care: the PROSPECT study. American Journal of Geriatric Psychiatry 2005;13(10):861-8.

Bogner HR, Lin JY, Morales KH. Patterns of early adherence to the antidepressant citalopram among older primary care patients: the prospect study. International Journal of Psychiatry in Medicine 2006;36(1):103-19.

Bogner HR, Morales KH, Post EP, Bruce ML. Diabetes, depression, and death: a randomized controlled trial of a depression treatment program for older adults based in primary care (PROSPECT). Diabetes Care 2007;30(12): 3005-10.

Bruce ML, Pearson JL. Designing an intervention to prevent suicide: PROSPECT (Prevention of suicide in primary care elderly: Collaborative trial). Dialogues in Clinical Neurosciences 1999;1(2):100-12.

* Bruce ML, Ten Have TR, Reynolds CF 3rd, Katz II, Schulberg HC, Mulsant BH, et al.Reducing suicidal ideation and depressive symptoms in depressed older primary care patients: a randomized controlled trial. JAMA 2004;291(9):1081-91.

Byers AL, Bruce ML, Raue P. Suicidal ideation in nondepressed elderly primary care patients: The PROSPECT Study [abstract]. American Journal of Geriatric Psychiatry 2009; 17:A86.

Gallo JJ, Bogner HR, Morales KH, Post EP, Lin JY, Bruce ML. The effect of a primary care practice-based depression intervention on mortality in older adults: a randomized trial. Annals of Internal Medicine 2007;146(10):689-98. Joo JH, Morales KH, de Vries HF, Gallo JJ. Disparity in use of psychotherapy offered in primary care between older African-American and white adults: results from a practice- 
based depression intervention trial. Journal of the American Geriatrics Society 2010;58(1):154-60.

Lin JY, Ten Have TR, Bogner H, Elliott MR. Baseline patient characteristics and mortality associated with longitudinal intervention compliance. Statistics in Medicine 2007;26(28):5100-15.

Lyness JM, Heo M, Datto CJ, Ten Have TR, Katz IR, Drayer $\mathrm{R}$, et al.Outcomes of minor and subsyndromal depression among elderly patients in primary care settings. Annals of Internal Medicine 2006;144(7):496-504.

Reynolds CF, Alexopoulos GS, Katz I, Bruce M, Schulberg HC, Tenhave T. Shifting the paradigm: Methodological and ethical questions in geriatric depression treatment research in the general medical sector. 39th Annual Meeting of the American College of Neuropsychopharmacology; 2000 Dec 10-14; San Juan; Puerto Rico. 2000:65.

Reynolds CF 3rd, Degenholtz H, Parker LS, Schulberg HC, Mulsant BH, Post E, et al.Treatment as usual (TAU) control practices in the PROSPECT Study: managing the interaction and tension between research design and ethics. International Journal of Geriatric Psychiatry 2001;16(6): 602-8.

Schulberg HC, Post EP, Raue PJ, Ten Have T, Miller M, Bruce ML. Treating late-life depression with interpersonal psychotherapy in the primary care sector. International Journal of Geriatric Psychiatry 2007;22(2):106-14. Wallace ML, Dombrovski AY, Morse JQ, Houck PR, Frank E, Alexopoulos GS, et al.Coping with health stresses and remission from late-life depression in primary care: A twoyear prospective study. International Journal of Geriatric Psychiatry 2012;27(2):178-86.

Williams JW Jr. Managed depression care reduces mortality in older adults with major depression. Evidence-Based Mental Health 2008;11(1):16.

Buszewicz 2010 \{published data only\}

* Buszewicz M, Griffin M, McMahon EM, Beecham J, King M. Evaluation of a system of structured, pro-active care for chronic depression in primary care: A randomised controlled trial [ISRCTN36610074]. Evidence-Based Mental Health 2010;10:ArtID 61.

Capoccia 2004 \{published data only\}

Boudreau DM, Capoccia KL, Sullivan SD, Blough DK, Ellsworth AJ, Clark DL, et al.Collaborative care model to improve outcomes in major depression. Annals of Pharmacotherapy 2002;36(4):585-91.

* Capoccia KL, Boudreau DM, Blough DK, Ellsworth AJ, Clark DR, Stevens NG, et al.Randomized trial of pharmacist interventions to improve depression care and outcomes in primary care. American Journal of HealthSystem Pharmacy 2004;61(4):364-72.

Chaney 2011 \{published data only\}

Chan D. Depression and comorbid PTSD in veterans: Evaluation of collaborative care programs and impact on utilization and costs [thesis]. Dissertation Abstracts International: Section B: The Sciences and Engineering 2008; 68(12B):7922.

Chan D, Cheadle AD, Reiber G, Untzer J, Chaney EF.
Health care utilization and its costs for depressed veterans with and without comorbid PTSD symptoms. Psychiatric Services 2009;60(12):1612-7.

* Chaney EF. Implementing collaborative care for depression treatment in primary care: A cluster randomized evaluation of a quality improvement practice redesign. Implementation Science 2011;6:121. [DOI: 10.1186/1748-5908-6-121] Chaney EF. Well-being among veterans enhancement study (WAVES) [NCT00105820]. ClinicalTrials.gov [www.clinicaltrials.gov] 2005.

Chew-Graham 2007 \{published data only\}

* Chew-Graham CA, Lovell K, Roberts C, Baldwin R, Morley M, Burns A, et al.A randomised controlled trial to test the feasibility of a collaborative care model for the management of depression in older people. British Journal of General Practice 2007;57(538):364-70.

Gilbody S. Depression in older adults: collaborative care model seems effective. Evidence-Based Mental Health 2008; 11(2): 44 .

Ciechanowski 2004 \{published data only\}

* Ciechanowski P, Wagner E, Schmaling K, Schwartz S, Williams B, Diehr P, et al.Community-integrated homebased depression treatment in older adults: a randomized controlled trial. JAMA 2004;291(13):1569-77.

Ciechanowski 2010 \{published data only\} Chaytor N, Ciechanowski P, Miller J, Fraser R, Russo J, Unutzer J, et al.Long-term outcomes from the PEARLS randomized trial for the treatment of depression in patients with epilepsy. Epilepsy and Behavior 2011;20(3):545-9.

* Ciechanowski P, Chaytor N, Miller J, Fraser R, Russo $\mathrm{J}$, Unutzer $\mathrm{J}$, et al.PEARLS depression treatment for individuals with epilepsy: A randomized controlled trial. Epilepsy and Behavior 2010;19(3):225-31.

Clarke 2005 \{published data only\}

* Clarke G, DeBar L, Lynch F, Powell J, Gale J, O’Connor E, et al.A randomized effectiveness trial of brief cognitivebehavioral therapy for depressed adolescents receiving antidepressant medication. Journal of the American Academy of Child and Adolescent Psychiatry 2005;44(9):888-98.

Cole 2006 \{published data only\}

* Cole MG, McCusker J, Elie M, Dendukuri N, Latimer E, Belzile E. Systematic detection and multidisciplinary care of depression in older medical inpatients: a randomized trial. Canadian Medical Association Journal 2006;174(1):38-44.

Datto 2003 \{published data only\}

* Datto CJ, Thompson R, Horowitz D, Disbot M, Oslin DW. The pilot study of a telephone disease management program for depression. General Hospital Psychiatry 2003; 25(3):169-77.

\section{Dietrich 2004 \{published data only\}}

Corson K, Gerrity MS, Dobscha SK. Screening for depression and suicidality in a VA primary care setting: 2 items are better than 1 item. American Journal of Managed Care 2004;10(11 Pt 2):839-45.

Dietrich AJ. Improving primary care for PTSD: Lessons from the RESPECT-Depression Project. 158th Annual 
Meeting of the American Psychiatric Association; 2005 May 21-26; Atlanta, GA. 2005:No. 23B.

* Dietrich AJ, Oxman TE, Williams JW, Schulberg HC, Bruce ML, Lee PW, et al.Re-engineering systems for the treatment of depression in primary care: cluster randomised controlled trial. BMJ 2004;329(7466):602.

Dietrich AJ, Oxman TE, Williams JW Jr, Kroenke K, Schulberg HC, Bruce M, et al.Going to scale: re-engineering systems for primary care treatment of depression. Annals of Family Medicine 2004;2(4):301-4.

Dobscha SK, Corson K, Gerrity M. Comorbid PTSD and alcohol abuse in veterans with elevated depression scores. 157th Annual Meeting of the American Psychiatric Association; 2004 May 1-6; New York, NY. 2004:NR555. Dobscha SK, Corson K, Solodky J, Gerrity MS. Use of videoconferencing for depression research: enrollment, retention, and patient satisfaction. Telemedicine Journal and E-Health 2005;11(1):84-9.

Kroenke K, Shen J, Oxman TE, Williams JW Jr, Dietrich AJ. Impact of pain on the outcomes of depression treatment: results from the RESPECT trial. Pain 2008;134(1-2): 209-15.

Lee PW, Dietrich AJ, Oxman TE, Williams JW Jr, Barry SL. Sustainable impact of a primary care depression intervention. Journal of the American Board of Family Medicine 2007;20(5):427-33.

Lee PW, Schulberg HC, Raue PJ, Kroenke K. Concordance between the PHQ-9 and the HSCL-20 in depressed primary care patients. Journal of Affective Disorders 2007;99(1-3): 139-45.

Nutting PA, Gallagher K, Riley K, White S, Dickinson WP, Korsen N, et al.Care management for depression in primary care practice: findings from the RESPECT-Depression trial. Annals of Family Medicine 2008;6(1):30-7.

Nutting PA, Gallagher KM, Riley K, White S, Dietrich AJ, Dickinson WP. Implementing a depression improvement intervention in five health care organizations: Experience from the RESPECT-Depression trial. Administration and Policy in Mental Health 2007;34(2):127-37.

Oxman TE, Schulberg EC, Greenberg RL, Dietrich AJ, Williams JW Jr, Nutting PA, et al.A fidelity measure for integrated management of depression in primary care. Medical Care 2006;44(11):1030-7.

Schulberg HC, Lee PW, Bruce ML, Raue PJ, Lefever JJ, Williams JW Jr, et al.Suicidal ideation and risk levels among primary care patients with uncomplicated depression. Annals of Family Medicine 2005;3(6):523-8.

Dwight-Johnson 2005 \{published data only\}

* Dwight-Johnson M, Ell K, Lee PJ. Can collaborative care address the needs of low-income Latinas with comorbid depression and cancer? Results from a randomized pilot study. Psychosomatics 2005;46(3):224-32.

Dwight-Johnson 2010 \{published and unpublished data\} * Dwight-Johnson M, Lagomasino IT, Hay J, Zhang L, Tang L, Green JM, et al.Effectiveness of collaborative care in addressing depression treatment preferences among lowincome Latinos. Psychiatric Services 2010;61(11):1112-8.
Dwight-Johnson 2011 \{published data only\}

* Dwight-Johnson M, Aisenberg E, Golinelli D, Hong S, O'Brien M, Ludman E. Telephone-based cognitivebehavioral therapy for Latino patients living in rural areas: A randomized pilot study. Psychiatric Services 2011;62(8): $936-42$.

Ell 2007 \{published data only\}

* Ell K, Unutzer J, Aranda M, Gibbs NE, Lee PJ, Xie B. Managing depression in home health care: a randomized clinical trial. Home Health Care Services Quarterly 2007;26 (3):81-104.

Ell 2008 \{published data only\}

Ell K, Quon B, Quinn DI, Dwight-Johnson M, Wells A, Lee $\mathrm{PJ}$, et al.Improving treatment of depression among lowincome patients with cancer: the design of the ADAPt-C study. General Hospital Psychiatry 2007;29(3):223-31. Ell K, Xie B, Kapetanovic S, Quinn DI, Lee PJ, Wells A, et al.One-year follow-up of collaborative depression care for low-income, predominantly Hispanic patients with cancer. Psychiatric Services 2011;62(2):162-70.

* Ell K, Xie B, Quon B, Quinn DI, Dwight-Johnson M, Lee PJ. Randomized controlled trial of collaborative care management of depression among low-income patients with cancer. Journal of Clinical Oncology 2008;26(27):4488-96.

Ell 2010 \{published data only\}

Ell K, Katon W, Cabassa LJ, Xie B, Lee PJ, Kapetanovic S, et al.Depression and diabetes among low-income Hispanics: design elements of a socioculturally adapted collaborative care model randomized controlled trial. International Journal of Psychiatry in Medicine 2009;39(2):113-32.

* Ell K, Katon W, Xie B, Lee PJ, Kapetanovic S, Guterman $\mathrm{J}$, et al.Collaborative care management of major depression among low-income, predominantly Hispanic subjects with diabetes: a randomized controlled trial. Diabetes Care 2010; 33(4):706-13.

Ell K, Katon W, Xie B, Lee PJ, Kapetanovic S, Guterman $\mathrm{J}$, et al.One-year postcollaborative depression care trial outcomes among predominantly Hispanic diabetes safety net patients. General Hospital Psychiatry 2011;33(5): 436-42.

Ell K, Lee PJ, Xie B. Depression care for low-income, minority, safety net clinic populations with comorbid illness. Research on Social Work Practice 2010;20(5):467-75. Hay JW, Katon WJ, Ell K, Lee PJ, Guterman JJ. Cost effectiveness analysis of collaborative care management of major depression among low-income, predominantly Hispanics with diabetes. Journal of Mental Health Policy and Economics. Proceedings of the 10th Workshop on Costs and Assessment in Psychiatry; 2011 Mar 25-27; Venice, Italy. 2011; Vol. 14:S11.

Palinkas LA, Ell K, Hansen M, Cabassa L, Wells A. Sustainability of collaborative care interventions in primary care settings. Journal of Social Work 2011;11(1):99-117.

Finley 2003 \{published data only\}

* Finley PR, Rens HR, Pont JT, Gess SL, Louie C, Bull SA, et al.Impact of a collaborative care model on depression 
in a primary care setting: A randomized controlled trial. Pharmacotherapy 2003;23(9):1175-85.

Fortney 2007 \{published data only\}

Deen TL, Fortney JC, Pyne JM. Relationship between satisfaction, patient-centered care, adherence and outcomes among patients in a collaborative care trial for depression. Administration and Policy in Mental Health and Mental Health Services Research 2011;38(5):345-55.

Edlund MJ, Fortney JC, Reaves CM, Pyne JM, Mittal D. Beliefs about depression and depression treatment among depressed veterans. Medical Care 2008;46(6):581-9. Fortney JC, Maclejewski ML, Tripathi SP, Deen TL, Pyne JM. A budget impact analysis of telemedicine-based collaborative care for depression. Medical Care 2011;49(9): 872-80.

Fortney JC, Pyne JM, Edlund MJ, Mittal D. Relationship between antidepressant medication possession and treatment response. General Hospital Psychiatry 2010;32(4): 377-9.

Fortney JC, Pyne JM, Edlund MJ, Robinson DE, Mittal D, Henderson KL. Design and implementation of the telemedicine-enhanced antidepressant management study. General Hospital Psychiatry 2006;28(1):18-26. Fortney JC, Pyne JM, Edlund MJ, Stecker T, Mittal $\mathrm{D}$, Robinson DE, et al.Reasons for antidepressant nonadherence among veterans treated in primary care clinics. Journal of Clinical Psychiatry 2011;72(6):827-34.

* Fortney JC, Pyne JM, Edlund MJ, Williams DK, Robinson DE, Mittal D, et al.A randomized trial of telemedicine-based collaborative care for depression. Journal of General Internal Medicine 2007;22(8):1086-93.

Fortney JC, Pyne JM, Steven CA, Williams JS, Hedrick RG, Lunsford AK, et al.A web-based clinical decision support system for depression care management. American Journal of Managed Care 2010;16(11):849-54.

Mittal D, Fortney JC, Pyne JM, Edlund MJ, Wetherell JL. Impact of comorbid anxiety disorders on healthrelated quality of life among patients with major depressive disorder. Psychiatric Services 2006;57(12):1731-7.

Mittal D, Fortney JC, Pyne JM, Wetherell JL. Predictors of persistence of comorbid generalized anxiety disorder among veterans with major depressive disorder. Journal of Clinical Psychiatry 2011;72(11):1445-51.

Pyne JM, Fortney JC, Tripathi SP, Maciejewski ML, Edlund MJ, Williams DK. Cost-effectiveness analysis of a rural telemedicine collaborative care intervention for depression. Archives of General Psychiatry 2010;67(8):812-21.

Fritsch 2007 \{published data only\}

* Fritsch R, Araya R, Solis J, Montt E, Pilowsky D, Rojas G. [A randomized trial of pharmacotherapy with telephone monitoring to improve treatment of depression in primary care in Santiago, Chile] [Spanish]. Revista Medica de Chile 2007;135(5):587-95.

Gensichen 2009 \{published data only\} Gensichen J, Guethlin C, Sarmand N, Sivakumaran D, Jager $\mathrm{C}$, Mergenthal K, et al.Patients' perspectives on depression case management in general practice - a qualitative study. Patient Education and Counseling 2012;86(1):114-9. Gensichen J, Guthlin C, Kleppel V, Jager C, Mergenthal $\mathrm{K}$, Gerlach FM, et al.Practice-based depression case management in primary care: A qualitative study on family doctors' perspectives. Family Practice 2011;28(5):565-71. Gensichen J, Jaeger C, Peitz M, Torge M, Guthlin C, Mergenthal $\mathrm{K}$, et al.Health care assistants in primary care depression management: Role perception, burdening factors, and disease conception. Annals of Family Medicine 2009;7(6):513-9.

Gensichen J, Petersen JJ, Karroum T, Rauck S, Ludman

E, Konig J, et al.Positive impact of a family practice-based depression case management on patient's self-management. General Hospital Psychiatry 2011;33(1):23-8.

Gensichen J, Torge M, Peitz M, Wendt-Hermainski $\mathrm{H}$, Beyer M, Rosemann T, et al.Case management for the treatment of patients with major depression in general practices - rationale, design and conduct of a cluster randomized controlled trial - PRoMPT (Primary care Monitoring for depressive Patient's Trial) [ISRCTN66386086] - study protocol. BMC Public Health 2005;5:101. [DOI: 10.1186/1471-2458-5-101]

* Gensichen J, von Korff M, Peitz M, Muth C, Beyer M, Guthlin C, et al.Case management for depression by health care assistants in small primary care practices: a cluster randomized trial. Annals of Internal Medicine 2009;151(6): 369-78.

Kendrick T. Healthcare assistant case management may reduce depression symptoms in primary care patients with major depression more than usual care. Evidence-Based Medicine 2010;15(1):10-1.

Gjerdingen 2009 \{published data only\}

* Gjerdingen D, Crow S, McGovern P, Miner M, Center B. Stepped care treatment of postpartum depression: impact on treatment, health, and work outcomes. Journal of the American Board of Family Medicine 2009;22(5):473-82.

Hedrick 2003 \{published data only\}

Goering P. Collaborative care speeds recovery from depression. Evidence-Based Mental Health 2003;6(4):116. Hedrick SC, Chaney EF. Effectiveness of team treatment of depression in primary care [NCT00012766]. ClinicalTrials.gov [www.clinicaltrials.gov] 2001.

* Hedrick SC, Chaney EF, Felker B, Liu CF, Hasenberg $\mathrm{N}$, Heagerty P, et al.Effectiveness of collaborative care depression treatment in veterans' affairs primary care. Journal of General Internal Medicine 2003;18(1):9-16. Lin P, Campbell DG, Chaney EF, Liu CF, Heagerty P, Felker $\mathrm{BL}$, et al.The influence of patient preference on depression treatment in primary care. Annals of Behavioral Medicine 2005;30(2):164-73.

Liu CF, Hedrick SC, Chaney EF, Heagerty P, Felker B, Hasenberg N, et al.Cost-effectiveness of collaborative care for depression in a primary care veteran population. Psychiatric Services 2003;54(5):698-704.

Hilty 2007 \{published data only\}

* Hilty D, Marks S, Wegelin J, Callahan EJ, Nesbitt 
TS. Randomized, controlled trial of disease management modules, including telepsychiatric care, for depression in rural primary care. Psychiatry 2007;4(2):58-65.

Huffman 2011 \{published data only\}

Celano CM, Mastromauro CA, Lenihan EC, Januzzi JL, Rollman BL, Huffman JC. Association of baseline anxiety with depression persistence at 6 months in patients with acute cardiac illness. Psychosomatic Medicine 2012;74(1): 93-9.

Huffman JC, Celano C, Mastromauro CA, Januzzi JL. Effects of baseline anxiety on depression persistence in a study of hospitalized cardiac patients. Psychosomatic Medicine. Proceedings from the 69th Annual Meeting of the American Psychosomatic Society; 2011 Mar 9-12; San Antonio, TX United States. 2011; Vol. 73, issue 3.

Huffman JC, Mastromauro CA, Sowden G, Fricchione GL, Healy BC, Januzzi JL. Impact of a depression care management program for hospitalized cardiac patients. Circulation. Cardiovascular Quality and Outcomes 2011;4 (2):198-205.

* Huffman JC, Mastromauro CA, Sowden GL, Wittmann C, Rodman R, Januzzi JL. A collaborative care depression management program for cardiac inpatients: Depression characteristics and in-hospital outcomes. Psychosomatics: Journal of Consultation Liaison Psychiatry 2011;52(1):26-33.

Hunkeler 2000 \{published data only\}

Hunkeler EM, Hargreaves WA, Meresman J. Efficacy of nurse tele-health care as an augmentation to ssri treatment of depression in primary care. 153rd Annual Meeting of the American Psychiatric Association; 2000 May 13-18; Chicago (IL). 2000:NR448.

Hunkeler EM, Hargreaves WA, Meresman J. Efficacy of nurse tele-health care as an augmentation to ssri treatment of depression in primary care. 155th Annual Meeting of the American Psychiatric Association; 2002 May 18-23rd; Philadelphia (PA). 2000:NR448.

${ }^{*}$ Hunkeler EM, Meresman JF, Hargreaves WA, Fireman B, Berman WH, Kirsch AJ, et al.Efficacy of nurse telehealth care and peer support in augmenting treatment of depression in primary care. Archives of Family Medicine 2000;9(8): $700-8$.

Meresman J, Hunkeler E, Hargreaves W. The nurse telecare project for treating depression: A progress report. 35th International Meeting of the Society for Psychotherapy Research; 2004 June 16-19; Rome. 2004:170.

Katon 1995a \{published data only\}

* Katon W, Von Korff M, Lin E, Walker E, Simon GE, Bush $T$, et al.Collaborative management to achieve treatment guidelines impact on depression in primary care. JAMA 1995;273(13):1026-31.

Katon 1995b \{published data only\}

Katon W, Von Korff M, Lin E, Walker E, Simon GE, Bush $\mathrm{T}$, et al.Collaborative management to achieve treatment guidelines impact on depression in primary care. JAMA 1995;273(13):1026-31.
Katon 1996a \{published data only\}

* Katon W, Robinson P, Von Korff M, Lin E, Bush T,

Ludman E, et al.A multifaceted intervention to improve treatment of depression in primary care. Archives of General Psychiatry 1996;53(10):924-32.

Robinson P, Katon W, Von Korff M, Bush T, Simon G, Lin E, et al.The education of depressed primary care patients: what do patients think of interactive booklets and a video?. Journal of Family Practice 1997;44(6):562-71.

Katon 1996b \{published data only\}

Katon W, Robinson P, Von Korff M, Lin E, Bush T, Ludman $\mathrm{E}$, et al.A multifaceted intervention to improve treatment of depression in primary care. Archives of General Psychiatry 1996;53(10):924-32.

Robinson P, Katon W, Von Korff M, Bush T, Simon G, Lin $\mathrm{E}$, et al.The education of depressed primary care patients: what do patients think of interactive booklets and a video?. Journal of Family Practice 1997;44(6):562-71.

\section{Katon 1999 \{published data only\}}

* Katon W, Von Korff M, Lin E, Simon G, Walker E, Unutzer J, et al.Stepped collaborative care for primary care patients with persistent symptoms of depression: a randomized trial. Archives of General Psychiatry 1999;56 (12):1109-15.

Katon WJ, Russo JE, Von Korff M, Lin EH, Ludman E, Ciechanowski PS. Long-term effects on medical costs of improving depression outcomes in patients with depression and diabetes. Diabetes Care 2008;31(6):1155-9.

Shaughnessy A. In patients who do not respond to antidepressants or who are at risk for recurrence, is collaborative care effective?. Evidence-Based Practice 2003;6 (2):9.

Simon GE, Katon WJ, VonKorff M, Unuetzer J, Lin $\mathrm{EH}$, Walker EA, et al.Cost-effectiveness of a collaborative care program for primary care patients with persistent depression. American Journal of Psychiatry 2001;158(10): 1638-44.

Unutzer J, Katon WJ, Russo J, Simon G, von Korff M, Lin E, et al.Willingness to pay for depression treatment in primary care. Psychiatric Services 2003;54(3):340-5. Walker EA, Koton WJ, Russo J, Von Korff M, Lin E, Simon $\mathrm{G}$, et al.Predictors of outcome in a primary care depression trial. Journal of General Internal Medicine 2000;15(12): 859-67.

\section{Katon 2001 \{published data only\}}

Bullock R. A depression relapse prevention programme improved adherence to medication and depressive symptoms but did not decrease relapses. Evidence-Based Mental Health 2001;4(4):113.

Gopinath S, Katon WJ, Russo JE, Ludman EJ. Clinical factors associated with relapse in primary care patients with chronic or recurrent depression. Journal of Affective Disorders 2007;101(1-3):57-63.

* Katon W, Rutter C, Ludman E J, Von Korff M, Lin E, Simon G, et al.A randomized trial of relapse prevention of 
depression in primary care. Archives of General Psychiatry 2001;58(3):241-7.

Lin EH, Von Korff M, Ludman EJ, Rutter C, Bush TM, Simon GE, et al.Enhancing adherence to prevent depression relapse in primary care. General Hospital Psychiatry 2003;25 (5):303-10.

Ludman E, Katon W, Bush T, Rutter C, Lin E, Simon G, et al.Behavioural factors associated with symptom outcomes in a primary care-based depression prevention intervention trial. Psychological Medicine 2003;33(6):1061-70.

Ludman E, Von Korff M, Katon W, Lin E, Simon G, Walker E, et al.The design, implementation, and acceptance of a primary care-based intervention to prevent depression relapse. International Journal of Psychiatry in Medicine 2000; 30(3):229-45.

Simon GE, Von Korff M, Ludman EJ, Katon WJ, Rutter C, Unutzer J, et al.Cost-effectiveness of a program to prevent depression relapse in primary care. Medical Care 2002;40 (10):941-50.

Von Korff M, Katon W, Rutter C, Ludman E, Simon G, Lin E, et al.Effect on disability outcomes of a depression relapse prevention program. Psychosomatic Medicine 2003; 65(6): $938-43$.

\section{Katon 2004 \{published data only\}}

Ciechanowski PS, Russo JE, Katon WJ. The difficult patient: A developmental perspective using an attachment theoretical framework. 63rd Annual Meeting of the American Psychosomatic Society; 2005 March 2-5; Vancouver, Canada. 2005:A122.

Ciechanowski PS, Russo JE, Katon WJ, Von Korff M, Simon GE, Lin EHB, et al.The association of patient relationship style and outcomes in collaborative care treatment for depression in patients with diabetes. Medical Care 2006;44(3):283-91.

Davidson MB, Echeverry D. Collaborative care for depression and chronic illnesses [comment]. New England Journal of Medicine 2011;364(13):1278; author reply 12789.

Gask L, Ludman E, Schaefer J. Qualitative study of an intervention for depression among patients with diabetes: how can we optimize patient-professional interaction?. Chronic Illness 2006;2(3):231-42.

Glasgow RE, Price DW. Individualised treatment improves depression in people with depression and diabetes. EvidenceBased Mental Health 2005;8(2):40.

Katon W, Russo J, Von Korff M, Lin E, Simon G, Bush T, et al.Long-term effects of a collaborative care intervention in persistently depressed primary care patients. Journal of General Internal Medicine 2002;17(10):741-8.

Katon W, Von Korff M, Lin E, Simon G, Ludman E, Bush $\mathrm{T}$, et al.Improving primary care treatment of depression among patients with diabetes mellitus: the design of the pathways study. General Hospital Psychiatry 2003;25(3): $158-68$.

Katon WJ, Von Korff M, Lin E, Simon G, Ciechanowski P, Ludman E, et al.The PATHWAYS study: A randomized trial of collaborative care in patients with diabetes and depression. 157th Annual Meeting of the American Psychiatric Association; 2004 May 1-6; New York (NY). 2004:No. 9A.

* Katon WJ, Von Korff M, Lin EH, Simon G, Ludman E, Russo J, et al.The Pathways Study: a randomized trial of collaborative care in patients with diabetes and depression. Archives of General Psychiatry 2004;61(10):1042-9.

Kinder LS, Katon WJ, Ludman E, Russo J, Simon G, Lin $\mathrm{EH}$, et al.Improving depression care in patients with diabetes and multiple complications. Journal of General Internal Medicine 2006;21(10):1036-41.

Lin EH, Katon W, Rutter C, Simon GE, Ludman EJ, Von Korff M, et al.Effects of enhanced depression treatment on diabetes self-care. Annals of Family Medicine 2006;4(1): 46-53.

Lin EH, Katon W, Rutter C, Von Korff M, Ludman EJ, Simon GE, et al.Effects of enhanced depression care on diabetes self management: A randomized-controlled trial. 63rd Annual Meeting of the American Psychosomatic Society; 2005 March 2-5; Vancouver, Canada. 2005:A23. O'Malley PG. Collaborative care for depression in patients with diabetes increased depression-free days and had economic benefit. ACP Journal Club 2007;146(3):78. Simon GE, Katon WJ, Lin EH, Rutter C, Manning WG, Von Korff M, et al.Cost-effectiveness of systematic depression treatment among people with diabetes mellitus. Archives of General Psychiatry 2007;64(1):65-72.

Upchurch SL. A collaborative care intervention improved depression outcomes, but not glycaemic control, in diabetes and comorbid depression. Evidence-Based Nursing 2005;8 (3):81.

\section{Katon 2010 \{published data only\}}

Hung W. Collaborative care for patients with depression improves chronic disease management: Commentary. Journal of Clinical Outcomes Management 2011;18(4): 156-7.

Katon W, Lin EH, Von Korff M, Ciechanowski P, Ludman E, Young B, et al.Integrating depression and chronic disease care among patients with diabetes and/or coronary heart disease: the design of the TEAMcare study. Contemporary Clinical Trials 2010;31(4):312-22.

Katon W, Russo J, Lin EHB, Schmittdiel J, Ciechanowski P, Ludman E, et al.Cost-effectiveness of a Multicondition Collaborative Care Intervention: A Randomized Controlled Trial. Archives of General Psychiatry 2012;69(5):506-514.

* Katon WJ, Lin EH, Von Korff M, Ciechanowski P, Ludman EJ, Young B, et al.Collaborative care for patients with depression and chronic illnesses. New England Journal of Medicine 2010;363(27):2611-20.

Katon WJ, Lin EHB, Von Korff M. The authors reply: "Collaborative care for depression and chronic illnesses" [Reply to comment]. New England Journal of Medicine 2011;364(13):1278-9.

McGregor M, Lin EH, Katon WJ. TEAMcare: an integrated multicondition collaborative care program for chronic illnesses and depression. Journal of Ambulatory Care 
Management 2011;34(2):152-62.

Von Korff M, Katon WJ, Lin EHB, Ciechanowski P, Peterson D, Ludman EJ, et al.Functional outcomes of multi-condition collaborative care and successful ageing: Results of randomised trial. BMJ 2011;343(7833):1083.

\section{Katzelnick 2000 \{published data only\}}

Katzelnick DJ, Simon GE, Pearson SD, Manning WG, Helstad CP, Henk HJ. Randomized trial of a depression management program in high utilizers of medical care. 151st Annual Meeting of the American Psychiatric Association; 1998 May 30- Jun 4; Toronto, Ontario, Canada. 1998.

* Katzelnick DJ, Simon GE, Pearson SD, Manning WG, Helstad CP, Henk HJ, et al.Randomized trial of a depression management program in high utilizers of medical care. Archives of Family Medicine 2000;9(4):345-51.

Katzelnick DJ, Simon GE, Pearson SD, Manning WG, Helstad CP, Henks HJ. Clinical outcomes care study. 152nd Annual Meeting of the American Psychiatric Association; 1999 May 15-20; Washington (DC). 1999.

Peveler R. A depression management programme reduced depression in frequent users of healthcare but did not reduce healthcare visits. Evidence-Based Mental Health 2001;4(3): 78-9.

Simon GE, Manning WG, Katzelnick DJ, Pearson SD, Henk HJ, Helstad CP. Cost-effectiveness of systematic depression treatment for high utilizers of general medical care. Archives of General Psychiatry 2001;58(2):181-7.

\section{Kroenke 2010 \{published data only\}}

Brown LF, Kroenke K, Theobald DE, Wu J, Tu W. The association of depression and anxiety with health-related quality of life in cancer patients with depression and/or pain. Psycho-Oncology 2010;19(7):734-41.

Johns SA, Kroenke K, Theobald DE, Wu J, Tu W. Telecare management of pain and depression in patients with cancer: patient satisfaction and predictors of use. Journal of Ambulatory Care Management 2011;34(2):126-39.

Kroenke K, Theobald D, Norton K, Sanders R, Schlundt S, McCalley $S$, et al.The Indiana Cancer Pain and Depression (INCPAD) trial Design of a telecare management intervention for cancer-related symptoms and baseline characteristics of study participants. General Hospital Psychiatry 2009;31(3):240-53.

Kroenke K, Theobald D, Wu J, Loza JK, Carpenter JS, $\mathrm{Tu} \mathrm{W}$. The association of depression and pain with healthrelated quality of life, disability, and health care use in cancer patients. Journal of Pain and Symptom Management 2010;40(3):327-41.

* Kroenke K, Theobald D, Wu J, Norton K, Morrison G, Carpenter J, et al.Effect of telecare management on pain and depression in patients with cancer. JAMA 2010;304(2): $163-71$.

Landis 2007 \{published data only\}

* Landis SE, Gaynes BN, Morrissey JP, Vinson N, Ellis AR, Domino ME. Generalist care managers for the treatment of depressed medicaid patients in North Carolina: a pilot study. BMC Family Practice 2007;8:7.
Lobello 2010 \{published data only\}

* Lobello K, Reddy S, Musgnung J, Pedersen R, Ninan P. Patient outcomes with education, drug therapy, and support: A study of venlafaxine ER-treated outpatients with major depressive disorder. Psychopharmacology Bulletin 2010;43(2):28-44.

Ludman 2007a \{published data only\} * Ludman EJ, Simon GE, Grothaus LC, Luce C, Markley DK, Schaefer J. A pilot study of telephone care management and structured disease self-management groups for chronic depression. Psychiatric Services 2007;58(8):1065-72.

Ludman 2007b \{published data only\} Ludman EJ, Simon GE, Grothaus LC, Luce C, Markley DK, Schaefer J. A pilot study of telephone care management and structured disease self-management groups for chronic depression. Psychiatric Services 2007;58(8):1065-72.

Ludman 2007c \{published data only\} Ludman EJ, Simon GE, Grothaus LC, Luce C, Markley DK, Schaefer J. A pilot study of telephone care management and structured disease self-management groups for chronic depression. Psychiatric Services 2007;58(8):1065-72.

Mann 1998 \{published data only\}

* Mann AH, Blizard R, Murray J, Smith JA, Botega N, MacDonald E, et al.An evaluation of practice nurses working with general practitioners to treat people with depression. British Journal of General Practice 1998;48(426): 875-9.

McCusker 2008 \{published data only\}

* McCusker J, Cole M, Yaffe M, Cappeliez P, Dawes M, Sewitch M, et al.Project direct: Pilot study of a collaborative intervention for depressed seniors. Canadian Journal of Community Mental Health 2008;27(2):201-18.

McMahon 2007 \{published data only\}

* McMahon L, Foran KM, Forrest SD, Taylor ML, Ingram

G, Rajwal M, et al.Graduate mental health worker case management of depression in UK primary care: a pilot study. British Journal of General Practice 2007;57(544): $880-5$.

\section{Oslin 2003 \{published data only\}}

Oslin D. Telephone disease management for depression and at-risk drinking. International Psychogeriatrics.

Proceedings of the International Psychogeriatric Association Eleventh International Congress; 2003 Aug 17-22, Chicago. 2003; Vol. 15.

* Oslin DW, Sayers S, Ross J, Kane V, Ten Have T, Conigliaro J, et al.Disease management for depression and at-risk drinking via telephone in an older population of veterans. Psychosomatic Medicine 2003;65(6):931-7.

\section{Patel 2010 \{published data only\}}

Patel V, Weiss H, Mann A. Predictors of outcome in patients with common mental disorders receiving a brief psychological treatment: An exploratory analysis of a randomized controlled trial from Goa, India. African Journal of Psychiatry (South Africa) 2010;13(4):291-6. * Patel V, Weiss HA, Chowdhary N, Naik S, Pednekar S, Chatterjee $S$, et al.Effectiveness of an intervention led by lay 
health counsellors for depressive and anxiety disorders in primary care in Goa, India (MANAS): A cluster randomised controlled trial. Lancet 2010;376(9758):2086-95.

Patel V, Weiss HA, Chowdhary N, Naik S, Pednekar S, Chatterjee $S$, et al.Lay health worker led intervention for depressive and anxiety disorders in India: impact on clinical and disability outcomes over 12 months. British Journal of Psychiatry 2011;199(6):459-66.

Patel VH, Kirkwood BR, Pednekar S, Araya R, King M, Chisholm D, et al.Improving the outcomes of primary care attenders with common mental disorders in developing countries: A cluster randomized controlled trial of a collaborative stepped care intervention in Goa, India. Trials 2008;9:4.

Piette 2011 \{published data only\}

Piette J, Duffy S, Torres T, Vogel M, Himle J, Richardson $\mathrm{C}$, et al.12-month outcomes from a randomized trial of telephone cognitive behavioral therapy for depressed patients with type 2 diabetes. Journal of General Internal Medicine. Proceedings of the 33rd Annual Meeting of the Society of General Internal Medicine; 2010 Apr 28-May 1, Minneapolis (MN) 2010;25(Suppl 3):S205.

* Piette JD, Richardson C, Himle J, Duffy S, Torres T, Vogel M, et al.A randomized trial of telephonic counseling plus walking for depressed diabetes patients. Medical Care 2011;49(7):641-8.

Pyne 2011 \{published data only\}

Curran GM, Pyne J, Fortney JC, Gifford A, Asch SM, Rimland D, et al.Development and implementation of collaborative care for depression in HIV clinics. AIDS Care - Psychological and Socio-Medical Aspects of AIDS/HIV 2011; 23(12):1626-36.

* Pyne JM, Fortney JC, Curran GM, Tripathi S, Atkinson $\mathrm{JH}$, Kilbourne AM, et al.Effectiveness of collaborative care for depression in human immunodeficiency virus clinics. Archives of Internal Medicine 2011;171(1):23-31.

Richards 2008a \{published data only\}

McMillan D, Gilbody S, Richards D. Defining successful treatment outcome in depression using the PHQ-9: A comparison of methods. Journal of Affective Disorders 2010; 127(1-3):122-9.

Richards D. Models of Stepped Care: evidence from the first UK trials of collaborative care for depression and stepped care for common mental health problems. 34th Annual Conference of the British Association for Behavioural and Cognitive Psychotherapies; 2006 July 19-21, Warwick. 2006:60.

Richards DA, Lovell K, Gilbody S, Gask L, Torgerson D, Barkham M, et al.Collaborative care for depression in UK primary care: A randomized controlled trial: Corrigendum. Psychological Medicine 2009;39(4):701.

* Richards DA, Lovell K, Gilbody S, Gask L, Torgerson $\mathrm{D}$, Barkham $\mathrm{M}$, et al.Collaborative care for depression in UK primary care: a randomized controlled trial [Erratum published in Psychological Medicine 2009;39(4):701]. Psychological Medicine 2008;38(2):279-87.

Simpson A, Richards D, Gask L, Hennessy S, Escott
D. Patients' experiences of receiving collaborative care for the treatment of depression in the UK: a qualitative investigation. Mental Health in Family Medicine 2008;5(2): 95-104.

\section{Richards 2008b \{published data only\}}

McMillan D, Gilbody S, Richards D. Defining successful treatment outcome in depression using the PHQ-9: A comparison of methods. Journal of Affective Disorders 2010; 127(1-3):122-9.

Richards D. Models of Stepped Care: evidence from the first UK trials of collaborative care for depression and stepped care for common mental health problems. 34th Annual Conference of the British Association for Behavioural and Cognitive Psychotherapies; 2006 July 19 - 21, Warwick. 2006:60.

Richards DA, Lovell K, Gilbody S, Gask L, Torgerson D, Barkham M, et al.Collaborative care for depression in UK primary care: A randomized controlled trial: Corrigendum. Psychological Medicine 2009;39(4):701.

* Richards DA, Lovell K, Gilbody S, Gask L, Torgerson $\mathrm{D}$, Barkham M, et al.Collaborative care for depression in UK primary care: a randomized controlled trial [Erratum published in Psychological Medicine 2009;39(4):701]. Psychological Medicine 2008;38(2):279-87.

Simpson A, Richards D, Gask L, Hennessy S, Escott D. Patients' experiences of receiving collaborative care for the treatment of depression in the UK: a qualitative investigation. Mental Health in Family Medicine 2008;5(2): 95-104.

\section{Richards 2012 \{published and unpublished data\}}

Richards DA. Multi-centre randomised controlled trial of collaborative care for depression [CADET (CollAborative DEpression Trial) [Is collaborative care more clinically and cost effective than usual care in the management of patients with moderate to severe depression in UK primary care?] [ ISRCTN32829227]. Current Controlled Trials [www.controlled-trials.com] 2009.

* Richards DA, Hughes-Morley A, Hayes RA, Araya R, Barkham M, Bland JM, et al.Collaborative depression trial (CADET): Multi-centre randomised controlled trial of collaborative care for depression - Study protocol. BMC Health Services Research 2009;16(9):188.

\section{Rojas 2007 \{published data only\}}

* Rojas G, Fritsch R, Solis J, Jadresic E, Castillo C, Gonzalez M, et al.Treatment of postnatal depression in lowincome mothers in primary-care clinics in Santiago, Chile: a randomised controlled trial. Lancet 2007;370(9599): 1629-37.

Zayas LH. Six-month multicomponent intervention improves postnatal depression in low-income settings. Evidence-Based Mental Health 2008;11(3):80.

Rollman 2005 \{published data only\}

Rollman B, Mazumdar S, Belnap B, Houck P, Lenze E, Schulberg H. Main outcomes from the relax trial of telephone delivered collaborative care for panic and generalized anxiety disorder. Journal of General Internal Medicine. Proceedings of the Annual Meeting of the 
Society of General Internal Medicine Minneapolis; 2010

April 28th-May 1st. 2010:S326.

* Rollman BL, Belnap BH, Mazumdar S, Houck PR, Zhu F,

Gardner W, et al.A randomized trial to improve the quality of treatment for panic and generalized anxiety disorders in primary care. Archives of General Psychiatry 2005;62(12): 1332-41.

Rollman BL, Belnap BH, Mazumdar S, Zhu F, Kroenke K, Schulberg HC, et al.Symptomatic severity of prime-MD diagnosed episodes of panic and generalized anxiety disorder in primary care. Journal of General Internal Medicine 2005; 20(7):623-8.

Rollman BL, Herbeck Belnap B, Reynolds CF, Schulberg HC, Shear MK. A contemporary protocol to assist primary care physicians in the treatment of panic and generalized anxiety disorders. General Hospital Psychiatry 2003;25(2): 74-82.

Shear K, Belnap BH, Mazumdar S, Houck P, Rollman BL. Generalized anxiety disorder severity scale (GADSS): a preliminary validation study. Depression and Anxiety 2006; 23(2):77-82.

\section{Rollman 2009 \{published data only\}}

Gallagher R. Telephone-delivered collaborative care for post-CABG depression is more effective than usual care for improving quality of life related to mental health. EvidenceBased Nursing 2010;13(2):37.

Morone NE, Weiner DK, Belnap BH, Karp JF, Mazumdar $S$, Houck PR, et al.The impact of pain and depression on recovery after coronary artery bypass grafting. Psychosomatic Medicine 2010;72(7):620-5.

Rollman BL, Belnap BH, LeMenager MS, Mazumdar S, Houck PR, Counihan PJ, et al.Telephone-delivered collaborative care for treating post-CABG depression: a randomized controlled trial. JAMA 2009;302(19): 2095-103.

Rollman BL, Belnap BH, LeMenager MS, Mazumdar S, Schulberg HC, Reynolds CF 3rd. The Bypassing the Blues treatment protocol: stepped collaborative care for treating post-CABG depression. Psychosomatic Medicine 2009;71 (2):217-30.

* Rollman BL, Herbeck Belnap B, LeMenager MS, Mazumdar S, Houck PR, Counihan PJ, et al.Telephonedelivered collaborative care for treating post-CABG depression: a randomized controlled trial. JAMA 2009;302 (19):2095-103.

Schulberg HC, Belnap BH, Houck PR, Mazumdar S, Reynolds CF III, Rollman BL. Treating post-CABG depression with telephone-delivered collaborative care: Does patient age affect treatment and outcome?. The American Journal of Geriatric Psychiatry 2011;19(10): 871-80.

Sriwattanakomen R, Mazumdar S, Belnap B, Houck P, Reynolds C, Rollman B. The effect of comorbid anxiety on post-CABG depressed patients' mental health related quality of life. Journal of General Internal Medicine. Proceedings of the 33rd Annual Meeting of the Society of General Internal Medicine; 2010 Apr 28- May 1; Minneapolis
(MN). 2010:S401.

Tindle HA, Belnap BH, Hum B, Houck PR, Mazumdar $\mathrm{S}$, Scheier M, et al.Optimism, depression, and depression remission after CABG surgery. Psychosomatic Medicine. Proceedings of the 69th Annual Meeting of the American Psychosomatic Society; 2011 Mar 9-12; San Antonio (TX). 2011; Vol. 73:A49.

Tully PJ. Randomised controlled trial: telephone-delivered collaborative care for post-CABG depression is more effective than usual care for improving mental-health-related quality of life. Evidence-Based Medicine 2010;15(2):57-8.

\section{Ross 2008 \{published data only\}}

* Ross JT, TenHave T, Eakin AC, Difilippo S, Oslin DW. A randomized controlled trial of a close monitoring program for minor depression and distress. Journal of General Internal Medicine 2008;23(9):1379-85.

\section{Rost 2001a \{published data only\}}

Adams SJ, Xu S, Dong F, Fortney J, Rost K. Differential effectiveness of depression disease management for rural and urban primary care patients. Journal of Rural Health 2006;22(4):343-50.

Clever SL, Ford DE, Rubenstein LV, Rost KM, Meredith LS, Sherbourne CD, et al.Primary care patients' involvement in decision-making is associated with improvement in depression. Medical Care 2006;44(5):398-405.

Dickinson LM, Rost K, Nutting PA, Elliott CE, Keeley $\mathrm{RD}$, Pincus $\mathrm{H}$. RCT of a care manager intervention for major depression in primary care: 2-Year costs for patients with physical vs psychological complaints. Annals of Family Medicine 2005;3(1):15-22.

Keeley RD, Smith JL, Nutting PA, Miriam Dickinson L, Perry Dickinson W, Rost KM. Does a depression intervention result in improved outcomes for patients presenting with physical symptoms?. Journal of General Internal Medicine 2004;19(6):615-23.

Lo Sasso AT, Rost K, Beck A. Modeling the impact of enhanced depression treatment on workplace functioning and costs: a cost-benefit approach. Medical Care 2006;44 (4):352-8.

Nutting PA, Dickinson LM, Rubenstein LV, Keeley RD, Smith JL, Elliott CE. Improving detection of suicidal ideation among depressed patients in primary care. Annals of Family Medicine 2005;3(6):529-36.

Nutting PA, Rost K, Dickinson M, Werner JJ, Dickinson P, Smith JL, et al.Barriers to initiating depression treatment in primary care practice. Journal of General Internal Medicine 2002;17(2):103-11.

Pyne JM, Rost KM, Farahati F, Tripathi SP, Smith J, Williams DK, et al.One size fits some: the impact of patient treatment attitudes on the cost-effectiveness of a depression primary-care intervention. Psychological Medicine 2005;35 (6):839-54.

Pyne JM, Rost KM, Zhang M, Williams DK, Smith J, Fortney J. Cost-effectiveness of a primary care depression intervention. Journal of General Internal Medicine 2003;18 (6):432-41.

Pyne JM, Smith J, Fortney J, Zhang M, Williams DK, Rost 
K. Cost-effectiveness of a primary care intervention for depressed females. Journal of Affective Disorders 2003;74(1): 23-32.

Rost K, Adams S, Xu S, Dong F. Rural-urban differences in hospitalization rates of primary care patients with depression. Psychiatric Services 2007;58(4):503-8. Rost K, Nutting P, Smith J, Werner J, Duan N. Improving depression outcomes in community primary care practice: A randomized trial of the QuEST intervention. Journal of General Internal Medicine 2001;16(3):143-9.

* Rost K, Nutting P, Smith JL, Elliott CE, Dickinson M. Managing depression as a chronic disease: a randomised trial of ongoing treatment in primary care. BMJ 2002;325 (7370):934.

Rost K, Nutting PA, Smith J, Werner JJ. Designing and implementing a primary care intervention trial to improve the quality and outcome of care for major depression. General Hospital Psychiatry 2000;22(2):66-77.

Rost K, Pyne JM, Dickinson LM, LoSasso AT. Costeffectiveness of enhancing primary care depression management on an ongoing basis. Annals of Family Medicine 2005;3(1):7-14.

Rost K, Smith JL, Dickinson M. The effect of improving primary care depression management on employee absenteeism and productivity. A randomized trial. Medical Care 2004;42(12):1202-10.

Rost KM, Duan N, Rubenstein LV, Ford DE, Sherbourne $\mathrm{CD}$, Meredith LS, et al.The Quality Improvement for Depression collaboration: general analytic strategies for a coordinated study of quality improvement in depression care. General Hospital Psychiatry 2001;23(5):239-53. Rost KM, Nutting P, Smith J, Werner J. Primary care training improves depression outcomes. 153rd Annual Meeting of the American Psychiatric Association; 2000 May 13-18, Chicago (IL). 2000:No.36E.

Smith JL, Rost KM, Nutting PA, Elliott CE. Resolving disparities in antidepressant treatment and quality-of-life outcomes between uninsured and insured primary care patients with depression. Medical Care 2001;39(9):910-22. Smith JL, Rost KM, Nutting PA, Elliott CE, Dickinson LM. Impact of ongoing primary care intervention on long term outcomes in uninsured and insured patients with depression. Medical Care 2002;40(12):1210-22. Smith JL, Rost KM, Nutting PA, Elliott CE, Duan N. A primary care intervention for depression. Journal of Rural Health 2000;16(4):313-23.

Xu S, Rost K, Dong F, Dickinson LM. Stakeholder benefit from depression disease management: Differences by rurality?. The Journal of Behavioral Health Services and Research 2011;38(1):114-21.

\section{Rost 2001b \{published data only\}}

Adams SJ, Xu S, Dong F, Fortney J, Rost K. Differential effectiveness of depression disease management for rural and urban primary care patients. Journal of Rural Health 2006;22(4):343-50.

Clever SL, Ford DE, Rubenstein LV, Rost KM, Meredith LS, Sherbourne CD, et al.Primary care patients' involvement in decision-making is associated with improvement in depression. Medical Care 2006;44(5):398-405.

Dickinson LM, Rost K, Nutting PA, Elliott CE, Keeley $\mathrm{RD}$, Pincus $\mathrm{H}$. RCT of a care manager intervention for major depression in primary care: 2-Year costs for patients with physical vs psychological complaints. Annals of Family Medicine 2005;3(1):15-22.

Keeley RD, Smith JL, Nutting PA, Miriam Dickinson L, Perry Dickinson W, Rost KM. Does a depression intervention result in improved outcomes for patients presenting with physical symptoms?. Journal of General Internal Medicine 2004;19(6):615-23.

Lo Sasso AT, Rost K, Beck A. Modeling the impact of enhanced depression treatment on workplace functioning and costs: a cost-benefit approach. Medical Care 2006;44 (4):352-8.

Nutting PA, Dickinson LM, Rubenstein LV, Keeley RD, Smith JL, Elliott CE. Improving detection of suicidal ideation among depressed patients in primary care. Annals of Family Medicine 2005;3(6):529-36.

Nutting PA, Rost K, Dickinson M, Werner JJ, Dickinson P, Smith JL, et al.Barriers to initiating depression treatment in primary care practice. Journal of General Internal Medicine 2002;17(2):103-11.

Pyne JM, Rost KM, Farahati F, Tripathi SP, Smith J, Williams DK, et al.One size fits some: the impact of patient treatment attitudes on the cost-effectiveness of a depression primary-care intervention. Psychological Medicine 2005;35 (6):839-54

Pyne JM, Rost KM, Zhang M, Williams DK, Smith J, Fortney J. Cost-effectiveness of a primary care depression intervention. Journal of General Internal Medicine 2003;18 (6):432-41.

Pyne JM, Smith J, Fortney J, Zhang M, Williams DK, Rost $\mathrm{K}$. Cost-effectiveness of a primary care intervention for depressed females. Journal of Affective Disorders 2003;74(1): 23-32.

Rost K, Adams S, Xu S, Dong F. Rural-urban differences in hospitalization rates of primary care patients with depression. Psychiatric Services 2007;58(4):503-8.

Rost K, Nutting P, Smith J, Werner J, Duan N. Improving depression outcomes in community primary care practice: A randomized trial of the QuEST intervention. Journal of General Internal Medicine 2001;16(3):143-9.

Rost K, Nutting P, Smith JL, Elliott CE, Dickinson M. Managing depression as a chronic disease: a randomised trial of ongoing treatment in primary care. BMJ 2002;325 (7370):934.

Rost K, Nutting PA, Smith J, Werner JJ. Designing and implementing a primary care intervention trial to improve the quality and outcome of care for major depression. General Hospital Psychiatry 2000;22(2):66-77.

Rost K, Pyne JM, Dickinson LM, LoSasso AT. Costeffectiveness of enhancing primary care depression management on an ongoing basis. Annals of Family Medicine 2005;3(1):7-14.

Rost K, Smith JL, Dickinson M. The effect of improving 
primary care depression management on employee absenteeism and productivity. A randomized trial. Medical Care 2004;42(12):1202-10.

Rost KM, Duan N, Rubenstein LV, Ford DE, Sherbourne CD, Meredith LS, et al.The Quality Improvement for Depression collaboration: general analytic strategies for a coordinated study of quality improvement in depression care. General Hospital Psychiatry 2001;23(5):239-53. Rost KM, Nutting P, Smith J, Werner J. Primary care training improves depression outcomes. 153rd Annual Meeting of the American Psychiatric Association; 2000 May 13-18; Chicago (IL). 2000:No.36E.

Smith JL, Rost KM, Nutting PA, Elliott CE. Resolving disparities in antidepressant treatment and quality-of-life outcomes between uninsured and insured primary care patients with depression. Medical Care 2001;39(9):910-22. Smith JL, Rost KM, Nutting PA, Elliott CE, Dickinson LM. Impact of ongoing primary care intervention on long term outcomes in uninsured and insured patients with depression. Medical Care 2002;40(12):1210-22. Smith JL, Rost KM, Nutting PA, Elliott CE, Duan N. A primary care intervention for depression. Journal of Rural Health 2000;16(4):313-23.

Xu S, Rost K, Dong F, Dickinson LM. Stakeholder benefit from depression disease management: Differences by rurality?. The Journal of Behavioral Health Services and Research 2011;38(1):114-21.

\section{Roy-Byrne 2001 \{published data only\}}

Katon WJ, Roy-Byrne P, Russo J, Cowley D. Costeffectiveness and cost offset of a collaborative care intervention for primary care patients with panic disorder. Archives of General Psychiatry 2002;59(12):1098-104. Pieters G. Collaborative care led to greater recovery, improvement, and adherence than usual care at 12 months in panic disorder. Evidence-Based Mental Health 2002;5(2): 49.

Roy-Byrne P, Katon W, Cowley DS, Russo J. A randomized effectiveness trial for panic disorder in primary care. 39th Annual Meeting of the American College of Neuropsychopharmacology; 2000 Dec 10-14; San Juan; Puerto Rico. 2000:311.

Roy-Byrne PP, Cowley DS, Russo J, Cohen E, Michelson E, Katon WJ. Primary care panic: id by doctor versus screening. 153rd Annual Meeting of the American Psychiatric Association; 2000 May 13-18; Chicago (IL). 2000:NR599.

Roy-Byrne PP, Craske MG, Stein MB, Sullivan JG, Bystritsky A, Katon WJ, et al.Cognitive-behavior therapy and medication for primary care panic disorder: Sustained superiority to usual care. 157th Annual Meeting of the American Psychiatric Association; 2004 May 1-6; New York (NY). 2004:No. 9B.

* Roy-Byrne PP, Katon W, Cowley DS, Russo J. A randomized effectiveness trial of collaborative care for patients with panic disorder in primary care. Archives of General Psychiatry 2001;58(9):869-76.

Roy-Byrne PP, Katon W, Cowley DS, Russo JE, Cohen
E, Michelson E, et al.Panic disorder in primary care: Biopsychosocial differences between recognized and unrecognized patients. General Hospital Psychiatry 2000;22 (6):405-11.

Roy-Byrne PP, Russo J, Cowley DS, Katon WJ. Panic disorder in public sector primary care: clinical characteristics and illness severity compared with "mainstream" primary care panic disorder. Depression and Anxiety 2003;17(2): 51-7.

Roy-Byrne PP, Russo J, Cowley DS, Katon WJ.

Unemployment and emergency room visits predict poor treatment outcome in primary care panic disorder. Journal of Clinical Psychiatry 2003;64(4):383-9.

Roy-Byrne PP, Russo J, Katon WJ. Improving care for panic in primary care. 153rd Annual Meeting of the American Psychiatric Association; 2000 May 13-18; Chicago (IL). 2000:No. 36D.

\section{Roy-Byrne 2005 \{published data only\}}

Arch JJ, Craske MG, Stein MB, Sherbourne CD, RoyByrne PP. Correlates of alcohol use among anxious and depressed primary care patients. General Hospital Psychiatry 2006;28(1):37-42.

Bricker JB, Russo J, Stein MB, Sherbourne C, Craske M, Schraufnagel TJ, et al.Does occasional cannabis use impact anxiety and depression treatment outcomes?: Results from a randomized effectiveness trial. Depression and Anxiety 2007; 24(6):392-8.

Bystritsky A, Wagner AW, Russo JE, Stein MB, Sherbourne $\mathrm{CD}$, Craske MG, et al.Assessment of beliefs about psychotropic medication and psychotherapy: development of a measure for patients with anxiety disorders. General Hospital Psychiatry 2005;27(5):313-8.

Chavira DA, Stein MB, Golinelli D, Sherbourne CD, Craske MG, Sullivan G, et al.Predictors of clinical improvement in a randomized effectiveness trial for primary care patients with panic disorder. Journal of Nervous and Mental Disease 2009;197(10):715-21.

Craske MG, Edlund MJ, Sullivan G, Roy-Byrne P, Sherbourne C, Bystritsky A, et al.Perceived unmet need for mental health treatment and barriers to care among patients with panic disorder. Psychiatric Services 2005;56(8):988-94. Craske MG, Golinelli D, Stein MB, Roy-Byrne P, Bystritsky A, Sherbourne C. Does the addition of cognitive behavioral therapy improve panic disorder treatment outcome relative to medication alone in the primary-care setting?. Psychological Medicine 2005;35(11):1645-54.

Craske MG, Roy-Byrne P, Stein MB, Sullivan G, HazlettStevens H, Bystritsky A, et al.CBT intensity and outcome for panic disorder in a primary care setting. Behavior Therapy 2006;37(2):112-9.

Katon W, Russo J, Sherbourne C, Stein MB, Craske M, Fan $\mathrm{M}-\mathrm{Y}$, et al.Incremental cost-effectiveness of a collaborative care intervention for panic disorder. Psychological Medicine 2006;36(3):353-63.

Kumar S. Coordinated care consisting of cognitive behavioural therapy plus medication improves panic 
disorder. Evidence-Based Mental Health 2005;8(4):110. Mukherjee S, Sullivan G, Perry D, Verdugo B, MeansChristensen A, Schraufnagel T, et al.Adherence to treatment among economically disadvantaged patients with panic disorder. Psychiatric Services 2006;57(12):1745-50.

Roy-Byrne P, Sherbourne C, Miranda J, Stein M, Craske M, Golinelli D, et al.Poverty and response to treatment among panic disorder patients in primary care. American Journal of Psychiatry 2006;163(8):1419-25.

Roy-Byrne P, Stein MB, Russo J, Craske M, Katon W, Sullivan G, et al.Medical illness and response to treatment in primary care panic disorder. General Hospital Psychiatry 2005;27(4):237-43.

* Roy-Byrne PP, Craske MG, Stein MB, Sullivan G, Bystritsky A, Katon W, et al.A randomized effectiveness trial of cognitive-behavioral therapy and medication for primary care panic disorder. Archives of General Psychiatry 2005;62 (3):290-8.

Roy-Byrne PP, Sherbourne CD, Craske MG, Stein MB, Katon W, Sullivan G, et al.Moving treatment research from clinical trials to the real world. Psychiatric Services 2003;54 (3):327-32.

Wagner AW, Bystritsky A, Russo JE, Craske MG,

Sherbourne CD, Stein MB, et al.Beliefs about psychotropic medication and psychotherapy among primary care patients with anxiety disorders. Depression and Anxiety 2005;21(3): 99-105.

\section{Roy-Byrne 2010 \{published data only\}}

Craske MG, Stein MB, Sullivan G, Sherbourne C, Bystritsky A, Rose RD, et al.Disorder-specific impact of coordinated anxiety learning and management treatment for anxiety disorders in primary care. Archives of General Psychiatry 2011;68(4):378-88.

* Roy-Byrne P, Craske MG, Sullivan G, Rose RD, Edlund MJ, Lang AJ, et al.Delivery of evidence-based treatment for multiple anxiety disorders in primary care: A randomized controlled trial. JAMA 2010;303(19):1921-8.

Roy-Byrne PP, Sherbourne CD, Stein MB, Sullivan G, Edlund M, Craske MG, et al.Coordinated Anxiety Learning and Management (CALM): Improving primary care anxiety outcomes [Primary Care Intervention Strategy for Anxiety Disorders] [NCT00347269]. www.clinicaltrials.gov/ct2/ show/NCT00347269 (Accessed 3 May 2012).

Stein MB, Roy Byrne PP, Craske MG, Campbell Sills L, Lang AJ, Golinelli D, et al.Quality of and patient satisfaction with primary health care for anxiety disorders. Journal of Clinical Psychiatry 2011;72(7):970-6.

Sullivan G, Craske MG, Sherbourne C, Edlund MJ, Rose RD, Golinelli D, et al.Design of the Coordinated Anxiety Learning and Management (CALM) study: innovations in collaborative care for anxiety disorders. General Hospital Psychiatry 2007;29(5):379-87.

\section{Rubenstein 2002 \{published data only\}}

Clever SL, Ford DE, Rubenstein LV, Rost KM, Meredith LS, Sherbourne CD, et al.Primary care patients' involvement in decision-making is associated with improvement in depression. Medical Care 2006;44(5):398-405.

Nutting PA, Dickinson LM, Rubenstein LV, Keeley RD, Smith JL, Elliott CE. Improving detection of suicidal ideation among depressed patients in primary care. Annals of Family Medicine 2005;3(6):529-36.

Rost KM, Duan N, Rubenstein LV, Ford DE, Sherbourne CD, Meredith LS, et al.The Quality Improvement for Depression collaboration: general analytic strategies for a coordinated study of quality improvement in depression care. General Hospital Psychiatry 2001;23(5):239-53.

* Rubenstein LV, Parker LE, Meredith LS, Altschuler A, dePillis E, Hernandez J, et al.Understanding team-based quality improvement for depression in primary care. Health Services Research 2002;37(4):1009-29.

Simon 2000a \{published data only\}

* Simon GE, VonKorff M, Rutter C, Wagner E. Randomised trial of monitoring, feedback, and management of care by telephone to improve treatment of depression in primary care. BMJ 2000;320(7234):550-4.

Williams JW Jr. Feedback to physicians plus telephone care management improved outcomes in primary care patients with depression [comment]]. ACP Journal Club 2000;133 (2):73.

\section{Simon 2000b \{published data only\}}

Simon GE, VonKorff M, Rutter C, Wagner E. Randomised trial of monitoring, feedback, and management of care by telephone to improve treatment of depression in primary care. BMJ 2000;320(7234):550-4.

Williams JW Jr. Feedback to physicians plus telephone care management improved outcomes in primary care patients with depression [comment]]. ACP Journal Club 2000;133 (2):73.

\section{Simon 2004a \{published data only\}}

Ludman EJ, Simon GE, Tutty S, Von Korff M. A randomized trial of telephone psychotherapy and pharmacotherapy for depression: continuation and durability of effects. Journal of Consulting and Clinical Psychology 2007;75(2):257-66.

Simon GE, Ludman EJ, Rutter C. Incremental benefit and cost of telephone care management and telephone psychotherapy for depression in primary care. Archives of General Psychiatry 2009;66(10):1081-9.

* Simon GE, Ludman EJ, Tutty S, Operskalski B, Von Korff M. Telephone psychotherapy and telephone care management for primary care patients starting antidepressant treatment: a randomized controlled trial. JAMA 2004;292(8):935-42.

Tutty S, Ludman EJ, Simon G. Feasibility and acceptability of a telephone psychotherapy program for depressed adults treated in primary care. General Hospital Psychiatry 2005;27 (6):400-10

Simon 2004b \{published data only\} Ludman EJ, Simon GE, Tutty S, Von Korff M. A randomized trial of telephone psychotherapy and pharmacotherapy for depression: continuation and 
durability of effects. Journal of Consulting and Clinical Psychology 2007;75(2):257-66.

Simon GE, Ludman EJ, Rutter C. Incremental benefit and cost of telephone care management and telephone psychotherapy for depression in primary care. Archives of General Psychiatry 2009;66(10):1081-9.

Simon GE, Ludman EJ, Tutty S, Operskalski B, Von Korff M. Telephone psychotherapy and telephone care management for primary care patients starting antidepressant treatment: a randomized controlled trial. JAMA 2004;292(8):935-42.

Tutty S, Ludman EJ, Simon G. Feasibility and acceptability of a telephone psychotherapy program for depressed adults treated in primary care. General Hospital Psychiatry 2005;27 (6):400-10.

\section{Simon 2011 \{published data only\}}

Simon GE. Pilot trial of depression care management by electronic secure messaging [feasibility of depression care management by E-Mail] [NCT00755235]. www.clinicaltrials.gov/ct2/show/NCT00755235 (Accessed 3 May 2012).

* Simon GE, Ralston JD, Savarino J, Pabiniak C, Wentzel C, Operskalski BH. Randomized trial of depression followup care by online messaging. Journal of General Internal Medicine 2011;26(7):698-704.

Smit 2006a \{published data only\}

Conradi HJ, de Jonge P, Kluiter H, Smit A, van der Meer $\mathrm{K}$, Jenner JA, et al.Enhanced treatment for depression in primary care: long-term outcomes of a psycho-educational prevention program alone and enriched with psychiatric consultation or cognitive behavioral therapy. Psychological Medicine 2007;37(6):849-62.

de Jonge P, Conradi HJ, Kaptein KI, Bockting CL, Korf J, Ormel J. Duration of subsequent episodes and periods of recovery in recurrent major depression [INSTEL trial]. Journal of Affective Disorders 2010;125(1-3):141-5.

* Smit A, Kluiter H, Conradi HJ, van der Meer K, Tiemens $B G$, Jenner JA, et al.Short-term effects of enhanced treatment for depression in primary care: Results from a randomized controlled trial. Psychological Medicine 2006;36 (1):15-26.

Smit A, Tiemens BG, Ormel J, Kluiter H, Jenner JA, van de Meer $\mathrm{K}$, et al.Enhanced treatment for depression in primary care: First year results on compliance, self-efficacy, the use of antidepressants and contacts with the primary care physician. Primary Care and Community Psychiatry 2005;10 (2):39-49.

Stant AD, Ten Vergert EM, Kluiter H, Conradi HJ, Smit A, Ormel J. Cost-effectiveness of a psychoeducational relapse prevention program for depression in primary care. Journal of Mental Health Policy and Economics 2009;12(4):195-217, 220.

Smit 2006b \{published data only\}

Conradi HJ, de Jonge P, Kluiter H, Smit A, van der Meer $\mathrm{K}$, Jenner JA, et al.Enhanced treatment for depression in primary care: long-term outcomes of a psycho-educational prevention program alone and enriched with psychiatric consultation or cognitive behavioral therapy. Psychological Medicine 2007;37(6):849-62.

de Jonge P, Conradi HJ, Kaptein KI, Bockting CL, Korf J, Ormel J. Duration of subsequent episodes and periods of recovery in recurrent major depression [INSTEL trial]. Journal of Affective Disorders 2010;125(1-3):141-5.

Smit A, Kluiter H, Conradi HJ, van der Meer K, Tiemens BG, Jenner JA, et al.Short-term effects of enhanced treatment for depression in primary care: Results from a randomized controlled trial. Psychological Medicine 2006;36 (1):15-26.

Smit A, Tiemens BG, Ormel J, Kluiter H, Jenner JA, van de Meer $\mathrm{K}$, et al.Enhanced treatment for depression in primary care: First year results on compliance, self-efficacy, the use of antidepressants and contacts with the primary care physician. Primary Care and Community Psychiatry 2005;10 (2):39-49.

Stant AD, Ten Vergert EM, Kluiter H, Conradi HJ, Smit A, Ormel J. Cost-effectiveness of a psychoeducational relapse prevention program for depression in primary care. Journal of Mental Health Policy and Economics 2009;12(4):195-217, 220.

Smit 2006c \{published data only\}

Conradi HJ, de Jonge P, Kluiter H, Smit A, van der Meer $\mathrm{K}$, Jenner JA, et al.Enhanced treatment for depression in primary care: long-term outcomes of a psycho-educational prevention program alone and enriched with psychiatric consultation or cognitive behavioral therapy. Psychological Medicine 2007;37(6):849-62.

de Jonge P, Conradi HJ, Kaptein KI, Bockting CL, Korf J, Ormel J. Duration of subsequent episodes and periods of recovery in recurrent major depression [INSTEL trial]. Journal of Affective Disorders 2010;125(1-3):141-5.

Smit A, Kluiter H, Conradi HJ, van der Meer K, Tiemens $B G$, Jenner JA, et al.Short-term effects of enhanced treatment for depression in primary care: Results from a randomized controlled trial. Psychological Medicine 2006;36 (1):15-26.

Smit A, Tiemens BG, Ormel J, Kluiter H, Jenner JA, van de Meer $\mathrm{K}$, et al.Enhanced treatment for depression in primary care: First year results on compliance, self-efficacy, the use of antidepressants and contacts with the primary care physician. Primary Care and Community Psychiatry 2005;10 (2):39-49.

Stant AD, Ten Vergert EM, Kluiter H, Conradi HJ, Smit A, Ormel J. Cost-effectiveness of a psychoeducational relapse prevention program for depression in primary care. Journal of Mental Health Policy and Economics 2009;12(4):195-217, 220.

\section{Strong 2008 \{published data only\}}

Forchuk C. A nurse-delivered intervention was effective for depression in patients with cancer. Evidence-Based Nursing 2009;12(1): 17 .

* Strong V, Waters R, Hibberd C, Murray G, Wall L, Walker $\mathrm{J}$, et al.Management of depression for people with cancer (SMaRT oncology 1): a randomised trial [see comment]. 
Lancet 2008;372(9632):40-8.

van der Feltz-Cornelis CM. A nurse delivered management programme for depression in people with cancer reduces depressive symptoms compared with usual care. EvidenceBased Mental Health 2009;12(1):9.

Walker J, Sharpe M. Depression Care for People with Cancer: a collaborative care intervention. General Hospital Psychiatry 2009;31(5):436-41.

Walker M, Walker L, Walker A, Bateman J, Braid F, Hebblewhite C, et al.Preventing psychiatric morbidity in people with cancer. Psycho-Oncology. Proceedings of the 11th World Congress of Psycho-Oncology of the International Psycho-Oncology Society, IPOS; 2009 Jun 21-24; Vienna, Austria. 2009; Vol. 18:S238.

\section{Swindle 2003 \{published data only\}}

* Swindle RW, Rao JK, Helmy A, Plue L, Zhou XH, Eckert GJ, et al.Integrating clinical nurse specialists into the treatment of primary care patients with depression. International Journal of Psychiatry in Medicine 2003;33(1): 17-37.

Weinberger M. An integrated model of primary care in mental health. http://www.clinicaltrials.gov/ct2/show/ NCT00013260 (Accessed 3 May 2012).

Uebelacker 2011 \{published data only\}

* Uebelacker LA, Marootian BA, Tigue P, Haggarty R, Primack JM, Miller IW. Telephone depression care management for Latino Medicaid health plan members: A pilot randomized controlled trial. Journal of Nervous and Mental Disease 2011;199(9):678-83.

\section{Unutzer 2002 \{published data only\}}

Apesoa Varano EC, Hinton L, Barker JC, Unutzer J. Clinician approaches and strategies for engaging older men in depression care. American Journal of Geriatric Psychiatry 2010;18(7):586-95.

Arean PA, Ayalon L, Hunkeler E, Lin EH, Tang L, Harpole $\mathrm{L}$, et al.Improving depression care for older, minority patients in primary care. Medical Care 2005;43(4):381-90. Arean PA, Gum AM, Tang L, Unutzer J. Service use and outcomes among elderly persons with low incomes being treated for depression. Psychiatric Services 2007;58(8): 1057-64.

Bao Y, Casalino LP, Ettner SL, Bruce ML, Solberg LI, Unutzer J. Designing payment for collaborative care for depression in primary care. Health Services Research 2011; 46(5):1436-51.

Blasinsky M, Goldman HH, Unutzer J. Project IMPACT: a report on barriers and facilitators to sustainability. Administration and Policy in Mental Health 2006;33(6): 718-29.

Buist-Bouwman M A. Collaborative care management improves physical functioning in older people with depression. Evidence-Based Mental Health 2005;8(4):106. Chan D, Fan MY, Unutzer J. Long-term effectiveness of collaborative depression care in older primary care patients with and without PTSD symptoms. International Journal of Geriatric Psychiatry 2011;26(7):758-64.

Conn DK. Collaborative care depression management for older adults: level of comorbidity does not affect outcome. Evidence-Based Mental Health 2005;8(4):105.

Fann JR, Fan MY, Unutzer J. Improving primary care for older adults with cancer and depression. Journal of General Internal Medicine 2009;24(Suppl 2):S417-24.

Gensichen J. IMPACT collaborative care improves depression in elderly patients in primary care in the longer term. Evidence-Based Mental Health 2006;9(3):76.

Gilbody S M. IMPACT collaborative care programme reduces suicide ideation in depressed older adults. EvidenceBased Mental Health 2007;10(2):51.

Goldstein KM, Harpole LH, Stechuchak KM, Coffman CJ, Bosworth HB, Steffens DC, et al.Hormone therapy does not affect depression severity in older women. American Journal of Geriatric Psychiatry 2005;13(7):616-23.

Grypma L, Haverkamp R, Little S, Unutzer J. Taking an evidence-based model of depression care from research to practice: making lemonade out of depression. General Hospital Psychiatry 2006;28(2):101-7.

Gum AM, Arean PA, Hunkeler E, Tang L, Katon W, Hitchcock P, et al.Depression treatment preferences in older primary care patients. Gerontologist 2006;46(1):14-22. Hegel MT, Imming Jr, Cyr-Provost M, Noel PH, Arean PA, Unutzer J. Role of behavioral health professionals in a collaborative stepped care treatment model for depression in primary care: Project IMPACT. Families, Systems and Health 2002;20(3):265-77.

Hinton L, Zweifach M, Oishi S, Tang L, Unutzer J. Gender disparities in the treatment of late-life depression: Qualitative and quantitative findings from the IMPACT trial. American Journal of Geriatric Psychiatry 2006;14(10): 884-92.

Hunkeler EM, Katon W, Tang L, Williams JWJ, Kroenke $\mathrm{K}$, Lin $\mathrm{EH}$, et al.Long term outcomes from the IMPACT randomised trial for depressed elderly patients in primary care [comments in: Evid Based Ment Health. 2006 Aug; 9(3):76; PMID: 16868195 and BMJ. 2006 Feb 4;332 (7536):249-50; PMID: 16455698]. BMJ 2006;332(7536): 259-63.

Katon W, Unutzer J, Fan MY, Williams JWJ, Schoenbaum $\mathrm{M}$, Lin $\mathrm{EH}$, et al.Cost-effectiveness and net benefit of enhanced treatment of depression for older adults with diabetes and depression. Diabetes Care 2006;29(2):265-70. Katon WJ, Fan MY, Lin EH, Unutzer J. Depressive symptom deterioration in a large primary care-based elderly cohort. American Journal of Geriatric Psychiatry 2006;14(3): 246-54.

Lin EH, Katon W, Von Korff M, Tang L, Williams JW, $\mathrm{Jr}$, et al.Effect of improving depression care on pain and functional outcomes among older adults with arthritis: a randomized controlled trial. JAMA 2003;290(18):2428-9. Lin EH, Tang L, Katon W, Hegel MT, Sullivan MD, Unutzer J. Arthritis pain and disability: response to collaborative depression care. General Hospital Psychiatry 
2006;28(6):482-6.

Lin EHB. Depression and Osteoarthritis. American Journal of Medicine 2008;121(11 Suppl 2):S16-9.

Lowe B, Unutzer J, Callahan CM, Perkins AJ, Kroenke K. Monitoring depression treatment outcomes with the patient health questionnaire-9. Medical Care 2004;42(12): 1194-201.

Noel PH, Williams Jr JW, Unutzer J, Worcbel J, Lee S, Cornell J, et al.Depression and comorbid illness in elderly primary care patients: Impact on multiple domains of health status and well-being. Annals of Family Medicine 2004;2(6):555-62.

Oxman TE. Collaborative care may improve depression management in older adults. Evidence-Based Mental Health 2003;6(3):86.

Pigeon WR, Hegel M, Unutzer J, Fan MY, Sateia MJ, Lyness JM, et al.Is insomnia a perpetuating factor for latelife depression in the IMPACT cohort?. Sleep 2008;31(4): 481-8.

Price J. Collaborative care improves health outcomes in older people with depression and arthritis. Evidence-Based Mental Health 2004;7(2):45.

Simon G. Collaborative care for depression: is effective in older people, as the IMPACT trial shows. BMJ 2006;332 (7536):249-50.

Slimmer L. A collaborative care management programme in a primary care setting was effective for older adults with late life depression. Evidence-Based Nursing 2003;6(3):91.

Steffens DC, Snowden M, Fan MY, Hendrie H, Katon WJ, Unutzer J. Cognitive impairment and depression outcomes in the IMPACT study. American Journal of Geriatric Psychiatry 2006;14(5):401-9.

Tang L, Song J, Belin TR, Unutzer J. A comparison of imputation methods in a longitudinal randomized clinical trial. Statistics in Medicine 2005;24(14):2111-28.

Thielke SM, Fan MY, Sullivan M, Unutzer J. Pain limits the effectiveness of collaborative care for depression. American Journal of Geriatric Psychiatry 2007;15(8):699-707. Thompson A, Fan MY, Unutzer J, Katon W. One extra month of depression: The effects of caregiving on depression outcomes in the IMPACT trial. International Journal of Geriatric Psychiatry 2008;23(5):511-6.

Unutzer J. Improving depression care for older adults. 155th Annual Meeting of the American Psychiatric Association; 2002 May 18-23; Philadelphia (PA). 2002:No. 45D. Unutzer J, Choi Y, Cook I A, Oishi S. A Web-based data management system to improve care for depression in a multicenter clinical trial. Psychiatric Services 2002;53(6): 671 .

Unutzer J, Ferrell B, Lin EH, Marmon T. Pharmacotherapy of pain in depressed older adults. Journal of the American Geriatrics Society 2004;52(11):1916-22.

* Unutzer J, Katon W, Callahan CM, Williams JW Jr, Hunkeler E, Harpole L, et al.Collaborative care management of late-life depression in the primary care setting: a randomized controlled trial. JAMA 2002;288
(22):2836-45.

Unutzer J, Katon W, Callahan CM, Williams JW Jr, Hunkeler E, Harpole L, et al.Depression treatment in a sample of 1,801 depressed older adults in primary care. JAMA 2002;288(22):2836-45.

Unutzer J, Katon W, Williams JW Jr, Callahan CM, Harpole L, Hunkeler EM, et al.Improving primary care for depression in late life: the design of a multicenter randomized trial. Medical Care 2001;39(8):785-99.

Unutzer J, Katon WJ, Fan MY, Schoenbaum MC, Lin EH, Della Penna RD, et al.Long-term cost effects of collaborative care for late-life depression. American Journal of Managed Care 2008;14(2):95-100.

Unutzer J, Powers D, Katon W, Langston C. From establishing an evidence-based practice to implementation in real-world settings: IMPACT as a case study. Psychiatric Clinics of North America 2005;28(4):1079-92.

Unutzer J, Tang L, Oishi S, Katon W, Williams JWJ, Hunkeler E, et al.Reducing suicidal ideation in depressed older primary care patients. Journal of the American Geriatrics Society 2006;54(10):1550-6. van Leeuwen Williams E, Unutzer J, Lee S, Noel PH. Collaborative depression care for the old-old: findings from the IMPACT trial. American Journal of Geriatric Psychiatry 2009;17(12):1040-9.

Vannoy SD, Arean P, Untzer J. Advantages of using estimated depression-free days for evaluating treatment efficacy. Psychiatric Services 2010;61(2):160-3.

Vannoy SD, Duberstein P, Cukrowicz K, Lin E, Fan MY, Unutzer J. The relationship between suicide ideation and late-life depression. American Journal of Geriatric Psychiatry 2007;15(12):1024-33.

Voils C, Olsen M, Williams Jr J. Identifying depressed older adults in primary care: A secondary analysis of a multisite randomized controlled trial. Primary Care Companion to the Journal of Clinical Psychiatry 2008;10(1):9-14.

Williams JW Jr, Katon W, Lin EHB, Noel PH, Worchel J, Cornell J, et al.Improving patient care: The effectiveness of depression care management on diabetes-related outcomes in older patients. Annals of Internal Medicine 2004;140(12): 1015.

\section{Vera 2010 \{published data only\}}

Vera M, Juarbe D. Depression Treatment in General Medical Settings. http://www.clinicaltrials.gov/ct2/show/ NCT00797901 (Accessed 3 May 2012).

* Vera M, Perez-Pedrogo C, Huertas SE, Reyes-Rabanillo ML, Juarbe D, Huertas A, et al.Collaborative care for depressed patients with chronic medical conditions: A randomized trial in Puerto Rico. Psychiatric Services 2010; 61(2):144-50.

\section{Vlasveld 2011 \{published data only\}}

Vlasveld MC, Anema JR, Beekman AT, van Mechelen W, Hoedeman R, van Marwijk HW, et al.Multidisciplinary collaborative care for depressive disorder in the occupational health setting: design of a randomised controlled trial and cost-effectiveness study. BMC Health Services Research 2008; 
8:99.

* Vlasveld MC, van der Feltz-Cornelis CM, Ader HJ, Anema JR, Hoedeman R, van Mechelen W, et al.Collaborative care for major depressive disorder in an occupational healthcare setting. British Journal of Psychiatry 2011 Nov 24 [Epub ahead of print]. [DOI: 10.1192/bjp.bp.111.095687]

Waitzkin 2011 \{published data only\}

* Waitzkin H, Getrich C, Heying S, Rodriguez L, Parmar A, Willging $\mathrm{C}$, et al.Promotoras as mental health practitioners in primary care: A multi-method study of an intervention to address contextual sources of depression. Journal of Community Health 2011;36(2):316-31.

Wells 2000a \{published data only\}

Callahan CM, Kroenke K, Counsell SR, Hendrie HC, Perkins AJ, Katon W, et al.Treatment of depression improves physical functioning in older adults. Journal of the American Geriatrics Society 2005;53(3):367-73.

Clever SL, Ford DE, Rubenstein LV, Rost KM, Meredith LS, Sherbourne CD, et al.Primary care patients' involvement in decision-making is associated with improvement in depression. Medical Care 2006;44(5):398-405.

Dwight-Johnson M, Unutzer J, Sherbourne C, Tang L, Wells KB. Can quality improvement programs for depression in primary care address patient preferences for treatment?. Medical Care 2001;39(9):934-44.

Fraser SA, Kroenke K, Callahan CM, Hui SL, Williams JWJ, Unutzer J. Low yield of thyroid-stimulating hormone testing in elderly patients with depression. General Hospital Psychiatry 2004;26(4):302-9.

Halpern J, Johnson MD, Miranda J, Wells KB. The partners in care approach to ethics outcomes in quality improvement programs for depression. Psychiatric Services 2004;55(5): 532-9.

Harpole LH, Stechuchak KM, Saur CD, Steffens DC, Unutzer J, Oddone E. Implementing a disease management intervention for depression in primary care: a random work sampling study. General Hospital Psychiatry 2003;25(4): $238-45$.

Harpole LH, Williams Jr JW, Olsen MK, Stechuchak $\mathrm{KM}$, Oddone E, Callahan CM, et al.Improving depression outcomes in older adults with comorbid medical illness. General Hospital Psychiatry 2005;27(1):4-12.

Hegel MT, Unutzer J, Tang L, Arean PA, Katon W, Noel $\mathrm{PH}$, et al.Impact of comorbid panic and posttraumatic stress disorder on outcomes of collaborative care for late-life depression in primary care. American Journal of Geriatric Psychiatry 2005;13(1):48-58.

Jaycox LH, Miranda J, Meredith LS, Duan N, Benjamin $\mathrm{B}$, Wells K. Impact of a primary care quality improvement intervention on use of psychotherapy for depression. Mental Health Services Research 2003;5(2):109-20.

Johnson MD, Meredith LS, Hickey SC, Wells KB. Influence of patient preference and primary care clinician proclivity for watchful waiting on receipt of depression treatment. General Hospital Psychiatry 2006;28(5):379-86.

Katon WJ, Schoenbaum M, Fan MY, Callahan CM, Williams JJ, Hunkeler E, et al.Cost-effectiveness of improving primary care treatment of late-life depression. Archives of General Psychiatry 2005;62(12):1313-20.

Klap R, Tang L, Schell T, Wells K, Sherbourne C, Miranda J, et al.How quality improvement interventions for depression affect stigma concerns over time: A nine-year follow-up study. Psychiatric Services 2009;60(2):258-61.

Koike AK, Unutzer J, Wells KB. Improving the care for depression in patients with comorbid medical illness. American Journal of Psychiatry 2002;159(10):1738-45. Levine S, Unutzer J, Yip JY, Hoffing M, Leung M, Fan M$\mathrm{Y}$, et al.Physicians' satisfaction with a collaborative disease management program for late-life depression in primary care. General Hospital Psychiatry 2005;27(6):383-91. Masaquel A, Wells K, Ettner SL. How does the persistence of depression influence the continuity and type of health insurance and coverage limits on mental health therapy?. Journal of Mental Health Policy and Economics 2007;10(3): 133-44.

Meredith LS, Cheng WJY, Hickey SC, Dwight-Johnson M. Factors associated with primary care clinicians' choice of a watchful waiting approach to managing depression. Psychiatric Services 2007;58(1):72-8.

Meredith LS, Jackson-Triche M, Duan N, Rubenstein LV, Camp P, Wells KB. Quality improvement for depression enhances long-term treatment knowledge for primary care clinicians. Journal of General Internal Medicine. 2000;15 (12):868-77.

Meredith LS, Orlando M, Humphrey N, Camp P, Sherbourne CD. Are better ratings of the patient-provider relationship associated with higher quality care for depression?. Medical Care 2001;39(4):349-60.

Miranda J, Duan N, Sherbourne C, Schoenbaum M, Lagomasino I, Jackson-Triche M, et al.Improving care for minorities: can quality improvement interventions improve care and outcomes for depressed minorities? Results of a randomized, controlled trial. Health Services Research 2003; 38(2):613-30.

Miranda J, Schoenbaum M, Sherbourne C, Duan N, Wells $\mathrm{K}$. Effects of primary care depression treatment on minority patients' clinical status and employment. Archives of General Psychiatry 2004;61(8):827-34.

Oishi SM, Shoai R, Katon W, Callahan C, Unutzer J, Arean P, et al.Impacting late life depression: Integrating a depression intervention into primary care. Psychiatric Quarterly 2003;74(1):75-89.

Roeloffs C, Sherbourne C, Unutzer J, Fink A, Tang L, Wells KB. Stigma and depression among primary care patients. General Hospital Psychiatry 2003;25(5):311-5.

Rost KM, Duan N, Rubenstein LV, Ford DE, Sherbourne CD, Meredith LS, et al.The Quality Improvement for Depression collaboration: general analytic strategies for a coordinated study of quality improvement in depression care. General Hospital Psychiatry 2001;23(5):239-53.

Rubenstein LV, Jackson-Triche M, Unutzer J, Miranda J, Minnium K, Pearson ML, et al.Evidence-based care for depression in managed primary care practices. Health 
Affairs 1999;18(5):89-105.

Schoenbaum M, Belin T, Wells K. Improving primary care depression treatment: the effect on patients' employment. Association for Health Services Research 1999;16:87. Schoenbaum M, Miranda J, Sherbourne C, Duan N, Wells K. Cost-effectiveness of interventions for depressed Latinos. Journal of Mental Health Policy and Economics 2004;7(2): 69-76.

Schoenbaum M, Sherbourne C, Wells K. Gender patterns in cost effectiveness of quality improvement for depression: Results of a randomized, controlled trial. Journal of Affective Disorders 2005;87(2-3):319-25.

Schoenbaum M, Unutzer J, McCaffrey D, Duan N, Sherbourne C, Wells KB. The effects of primary care depression treatment on patients' clinical status and employment. Health Services Research 2002;37(5):1145-58. Schoenbaum M, Unutzer J, Sherbourne C, Duan N, Rubenstein LV, Miranda J, et al.Cost-effectiveness of practice-initiated quality improvement for depression: results of a randomized controlled trial. JAMA 2001;286 (11):1325-30.

Sherbourne CD, Edelen MO, Zhou A, Bird C, Duan $\mathrm{N}$, Wells KB. How a therapy-based quality improvement intervention for depression affected life events and psychological well-being over time: a 9-year longitudinal analysis. Medical Care 2008;46(1):78-84.

Sherbourne CD, Schoenbaum M, Wells KB, Croghan TW. Characteristics, treatment patterns, and outcomes of persistent depression despite treatment in primary care. General Hospital Psychiatry 2004;26(2):106-14.

Sherbourne CD, Weiss R, Duan N, Bird C, Wells K. Do the effects of quality improvement for depression differ for men and women?. Quality of Life Research 2003;12(7):767. Sherbourne CD, Weiss R, Duan N, Bird CE, Wells KB. Do the effects of quality improvement for depression care differ for men and women? Results of a group-level randomized controlled trial. Medical Care 2004;42(12):1186-93. Sherbourne CD, Wells KB, Duan N, Miranda J, Unutzer $\mathrm{J}$, Jaycox L, et al.Long-term effectiveness of disseminating quality improvement for depression in primary care. Archives of General Psychiatry 2001;58(7):696-703. Sherbourne DC, Unutzer J, Schoenbaum M, Duan N, Lenert LA, Sturm R, et al.Can utility-weighted healthrelated quality-of-life estimates capture health effects of quality improvement for depression?. Medical Care 2001; 39(11):1246-59.

Unutzer J. Collaborative care for late-life depression. International Psychogeriatrics. Proceedings of the International Psychogeriatric Association Eleventh International Congress; 2003 Aug 17-22 2003; Chicago (IL). 2003; Vol. 15.

Unutzer J. Collaborative care for late life depression in primary care: a randomized controlled trial. Proceedings of the 14th Annual Meeting of the American Association for Geriatric Psychiatry; 2001 23-26 Feb; San Francisco (CA). 2001.

Unutzer J, Rubenstein L, Katon WJ, Tang L, Duan
$\mathrm{N}$, Lagomasino IT, et al.Two-year effects of quality improvement programs on medication management for depression. Archives of General Psychiatry 2001;58(10): 935-42.

Velarde F, Lagomasino IT, Miranda J, Sherbourne C, Wells $\mathrm{KB}$. Impact of a depression intervention on US-born and immigrant Latinos. Proceedings of the 158th Annual Meeting of the American Psychiatric Association; 2005 May 21-26; Atlanta (GA). 2005:NR141.

Watkins KE, Paddock SM, Zhang L, Wells KB. Improving care for depression in patients with comorbid substance misuse. American Journal of Psychiatry 2006;163(1): 125-32.

Wells K, Sherbourne C, Duan N, Unutzer J, Miranda J, Schoenbaum M, et al.Quality improvement for depression in primary care: Do patients with subthreshold depression benefit in the long run?. American Journal of Psychiatry 2005;162(6): 1149-57.

Wells KB. The design of partners in care: Evaluating the cost-effectiveness of improving care for depression in primary care. Social Psychiatry and Psychiatric Epidemiology 1999;34(1):20-9.

Wells KB, Rubenstein LV, Unutzer J, Miranda J, Jackson M. Improving treatment resources for primary care depressed patients in managed care. Proceedings of the 151st Annual Meeting of the American Psychiatric Association; 1998 May 30-June 4; Toronto Ontario. 1998.

Wells KB, Schoenbaum M, Duan N, Miranda J, Tang L, Sherbourne C. Cost-effectiveness of quality improvement programs for patients with subthreshold depression or depressive disorder. Psychiatric Services 2007;58(10): 1269-78.

Wells KB, Sherbourne CD, Miranda J, Tang L, Benjamin $\mathrm{B}$, Duan N. The cumulative effects of quality improvement for depression on outcome disparities over 9 years: results from a randomized, controlled group-level trial. Medical Care 2007;45(11):1052-9.

Wells KB, Sherbourne CD, Schoenbaum M, Rubenstein LV, Duan N, Meredith L, et al.Improving quality of care, outcomes and employment of depressed primary care patients. Association for Health Services Research 1999;16:88. Wells KB Sherbourne CD Schoenbaum N Duan N Meredith LS Unutzer J Miranda J Carney M Rubenstein LV. Impact of disseminating quality improvement programs for depression in managed primary care: a randomized controlled trial [erratum appears in JAMA 2000 Jun 28;283 (24):3204]. JAMA 2000;283(2):212-20.

Wells K Sherbourne C Schoenbaum M Ettner S Duan N Miranda J, et al.Five-year impact of quality improvement for depression: results of a group-level randomized controlled trial. Archives of General Psychiatry 2004;61(4):378-86. Williams JW Jr, Katon W, Lin EH, Noel PH, Worchel J, Cornell J, et al.The effectiveness of depression care management on diabetes-related outcomes in older patients. Annals of Internal Medicine 2004;140(12):1015-24.

Wells 2000b \{published data only\} Callahan CM, Kroenke K, Counsell SR, Hendrie HC, 
Perkins AJ, Katon W, et al.Treatment of depression improves physical functioning in older adults. Journal of the American Geriatrics Society 2005;53(3):367-73.

Clever SL, Ford DE, Rubenstein LV, Rost KM, Meredith LS, Sherbourne CD, et al.Primary care patients' involvement in decision-making is associated with improvement in depression. Medical Care 2006;44(5):398-405.

Dwight-Johnson M, Unutzer J, Sherbourne C, Tang L, Wells KB. Can quality improvement programs for depression in primary care address patient preferences for treatment?. Medical Care 2001;39(9):934-44.

Fraser SA, Kroenke K, Callahan CM, Hui SL, Williams JWJ, Unutzer J. Low yield of thyroid-stimulating hormone testing in elderly patients with depression. General Hospital Psychiatry 2004;26(4):302-9.

Halpern J, Johnson MD, Miranda J, Wells KB. The partners in care approach to ethics outcomes in quality improvement programs for depression. Psychiatric Services 2004;55(5): 532-9.

Harpole LH, Stechuchak KM, Saur CD, Steffens DC, Unutzer J, Oddone E. Implementing a disease management intervention for depression in primary care: a random work sampling study. General Hospital Psychiatry 2003;25(4): 238-45.

Harpole LH, Williams Jr JW, Olsen MK, Stechuchak $\mathrm{KM}$, Oddone E, Callahan CM, et al.Improving depression outcomes in older adults with comorbid medical illness. General Hospital Psychiatry 2005;27(1):4-12.

Hegel MT, Unutzer J, Tang L, Arean PA, Katon W, Noel $\mathrm{PH}$, et al.Impact of comorbid panic and posttraumatic stress disorder on outcomes of collaborative care for late-life depression in primary care. American Journal of Geriatric Psychiatry 2005;13(1):48-58.

Jaycox LH, Miranda J, Meredith LS, Duan N, Benjamin B, Wells K. Impact of a primary care quality improvement intervention on use of psychotherapy for depression. Mental Health Services Research 2003;5(2):109-20.

Johnson MD, Meredith LS, Hickey SC, Wells KB. Influence of patient preference and primary care clinician proclivity for watchful waiting on receipt of depression treatment. General Hospital Psychiatry 2006;28(5):379-86.

Katon WJ, Schoenbaum M, Fan MY, Callahan CM, Williams JJ, Hunkeler E, et al.Cost-effectiveness of improving primary care treatment of late-life depression. Archives of General Psychiatry 2005;62(12):1313-20. Klap R, Tang L, Schell T, Wells K, Sherbourne C, Miranda J, et al.How quality improvement interventions for depression affect stigma concerns over time: A nine-year follow-up study. Psychiatric Services 2009;60(2):258-61.

Koike AK, Unutzer J, Wells KB. Improving the care for depression in patients with comorbid medical illness. American Journal of Psychiatry 2002;159(10):1738-45. Levine S, Unutzer J, Yip JY, Hoffing M, Leung M, Fan M$\mathrm{Y}$, et al.Physicians' satisfaction with a collaborative disease management program for late-life depression in primary care. General Hospital Psychiatry 2005;27(6):383-91. Masaquel A, Wells K, Ettner SL. How does the persistence of depression influence the continuity and type of health insurance and coverage limits on mental health therapy?. Journal of Mental Health Policy and Economics 2007;10(3): 133-44.

Meredith LS, Cheng WJY, Hickey SC, Dwight-Johnson M. Factors associated with primary care clinicians' choice of a watchful waiting approach to managing depression. Psychiatric Services 2007;58(1):72-8.

Meredith LS, Jackson-Triche M, Duan N, Rubenstein LV, Camp P, Wells KB. Quality improvement for depression enhances long-term treatment knowledge for primary care clinicians. Journal of General Internal Medicine 2000;15 (12):868-77.

Meredith LS, Orlando M, Humphrey N, Camp P, Sherbourne CD. Are better ratings of the patient-provider relationship associated with higher quality care for depression?. Medical Care 2001;39(4):349-60.

Miranda J, Duan N, Sherbourne C, Schoenbaum M, Lagomasino I, JacksonTriche M, et al.Improving care for minorities: can quality improvement interventions improve care and outcomes for depressed minorities? Results of a randomized, controlled trial. Health Services Research 2003; 38(2):613-30.

Miranda J, Schoenbaum M, Sherbourne C, Duan N, Wells K. Effects of primary care depression treatment on minority patients' clinical status and employment. Archives of General Psychiatry 2004;61(8):827-34.

Oishi SM, Shoai R, Katon W, Callahan C, Unutzer J, Arean $\mathrm{P}$, et al.Impacting late life depression: Integrating a depression intervention into primary care. Psychiatric Quarterly 2003;74(1):75-89.

Roeloffs C, Sherbourne C, Unutzer J, Fink A, Tang L, Wells KB. Stigma and depression among primary care patients. General Hospital Psychiatry 2003;25(5):311-5.

Rost KM, Duan N, Rubenstein LV, Ford DE, Sherbourne CD, Meredith LS, et al.The Quality Improvement for Depression collaboration: general analytic strategies for a coordinated study of quality improvement in depression care. General Hospital Psychiatry 2001;23(5):239-53. Rubenstein LV, Jackson-Triche M, Unutzer J, Miranda J, Minnium K, Pearson ML, et al.Evidence-based care for depression in managed primary care practices. Health Affairs 1999;18(5):89-105.

Schoenbaum M, Belin T, Wells K. Improving primary care depression treatment: the effect on patients' employment. Association for Health Services Research 1999;16:87.

Schoenbaum M, Miranda J, Sherbourne C, Duan N, Wells K. Cost-effectiveness of interventions for depressed Latinos. Journal of Mental Health Policy and Economics 2004;7(2): 69-76.

Schoenbaum M, Unutzer J, McCaffrey D, Duan N, Sherbourne C, Wells KB. The effects of primary care depression treatment on patients' clinical status and employment. Health Services Research 2002;37(5):1145-58. Schoenbaum M, Unutzer J, Sherbourne C, Duan N, Rubenstein LV, Miranda J, et al.Cost-effectiveness of practice-initiated quality improvement for depression: 
results of a randomized controlled trial. JAMA 2001;286 (11):1325-30

Schoenbaum M Sherbourne C Wells K. Gender patterns in cost effectiveness of quality improvement for depression: Results of a randomized, controlled trial. Journal of Affective Disorders 2005;87(2-3):319-25.

Sherbourne CD, Edelen MO, Zhou A, Bird C, Duan $\mathrm{N}$, Wells KB. How a therapy-based quality improvement intervention for depression affected life events and psychological well-being over time: a 9-year longitudinal analysis. Medical Care 2008;46(1):78-84.

Sherbourne CD, Schoenbaum M, Wells KB, Croghan

TW. Characteristics, treatment patterns, and outcomes of persistent depression despite treatment in primary care. General Hospital Psychiatry 2004;26(2):106-14.

Sherbourne CD, Weiss R, Duan N, Bird C, Wells K. Do the effects of quality improvement for depression differ for men and women?. Quality of Life Research 2003;12(7):767. Sherbourne CD, Weiss R, Duan N, Bird CE, Wells KB. Do the effects of quality improvement for depression care differ for men and women? Results of a group-level randomized controlled trial. Medical Care 2004;42(12):1186-93.

Sherbourne CD, Wells KB, Duan N, Miranda J, Unutzer J, Jaycox L, et al.Long-term effectiveness of disseminating quality improvement for depression in primary care. Archives of General Psychiatry 2001;58(7):696-703.

Sherbourne DC, Unutzer J, Schoenbaum M, Duan N, Lenert LA, Sturm R, et al.Can utility-weighted healthrelated quality-of-life estimates capture health effects of quality improvement for depression?. Medical Care 2001; 39(11):1246-59.

Unutzer J. Collaborative care for late life depression in primary care: a randomized controlled trial. Proceedings of the 14th Annual Meeting of the American Association for Geriatric Psychiatry; 2001 23-26 February; San Francisco (CA). 2001.

Unutzer J. Collaborative care for late-life depression. International Psychogeriatrics. Proceedings of the International Psychogeriatric Association Eleventh International Congress; 2003 Aug 17-22; Chicago (IL). 2003

Unutzer J, Rubenstein L, Katon WJ, Tang L, Duan $\mathrm{N}$, Lagomasino IT, et al.Two-year effects of quality improvement programs on medication management for depression. Archives of General Psychiatry 2001;58(10): 935-42.

Velarde F, Lagomasino IT, Miranda J, Sherbourne C, Wells $\mathrm{KB}$. Impact of a depression intervention on US-born and immigrant Latinos. Proceedings of the 158th Annual Meeting of the American Psychiatric Association; 2005 May 21-26; Atlanta (GA). 2005.

Watkins KE, Paddock SM, Zhang L, Wells KB. Improving care for depression in patients with comorbid substance misuse. American Journal of Psychiatry 2006;163(1): $125-32$.

Wells K, Sherbourne C, Duan N, Unutzer J, Miranda J, Schoenbaum M, et al.Quality improvement for depression in primary care: Do patients with subthreshold depression benefit in the long run?. American Journal of Psychiatry 2005;162(6):1149-57.

Wells K, Sherbourne C, Schoenbaum M, Ettner S, Duan N Miranda J, et al.Five-year impact of quality improvement for depression: results of a group-level randomized controlled trial. Archives of General Psychiatry 2004;61(4):378-86. Wells KB. The design of partners in care: Evaluating the cost-effectiveness of improving care for depression in primary care. Social Psychiatry and Psychiatric Epidemiology 1999;34(1):20-9.

Wells KB, Rubenstein LV, Unutzer J, Miranda J, Jackson M. Improving treatment resources for primary care depressed patients in managed care. Proceedings of the 151st Annual Meeting of the American Psychiatric Association; 1998 May 30-June 4; Toronto (Ontario). 1998.

Wells KB, Schoenbaum M, Duan N, Miranda J, Tang L, Sherbourne C. Cost-effectiveness of quality improvement programs for patients with subthreshold depression or depressive disorder. Psychiatric Services 2007;58(10): 1269-78.

Wells KB, Sherbourne CD, Miranda J, Tang L, Benjamin $\mathrm{B}$, Duan $\mathrm{N}$. The cumulative effects of quality improvement for depression on outcome disparities over 9 years: results from a randomized, controlled group-level trial. Medical Care 2007;45(11):1052-9.

Wells KB, Sherbourne CD, Schoenbaum M, Rubenstein LV, Duan N, Meredith L, et al.Improving quality of care, outcomes and employment of depressed primary care patients. Association for Health Services Research 1999;16:88. * Wells KB, Sherbourne CD, Schoenbaum N, Duan N, Meredith LS, Unutzer J, et al.Impact of disseminating quality improvement programs for depression in managed primary care: a randomized controlled trial [erratum appears in JAMA 2000 Jun 28;283(24):3204]. JAMA 2000; 283(2):212-20.

Williams JW Jr, Katon W, Lin EH, Noel PH, Worchel $\mathrm{J}$, Cornell J, et al.The effectiveness of depression care management on diabetes-related outcomes in older patients. Annals of Internal Medicine 2004;140(12):1015-24.

Wilkinson 1993 \{published data only\}

Wilkinson G. The role of the practice nurse in the management of depression. International Review of Psychiatry 1992;4(3-4):311-5.

* Wilkinson G, Allen P, Marshall E, Walker J, Browne W, Mann AH. The role of the practice nurse in the management of depression in general-practice: treatment adherence to antidepressant medication. Psychological Medicine 1993;23(1):229-37.

Williams 2007 \{published data only\}

* Williams LS, Kroenke K, Bakas T, Plue LD, Brizendine E, $\mathrm{Tu}$ W, et al.Care management of poststroke depression: a randomized, controlled trial. Stroke 2007;38(3):998-1003.

Yeung 2010 \{published data only\}

* Yeung A, Shyu I, Fisher L, Wu S, Yang H, Fava M. Culturally sensitive collaborative treatment for depressed 
Chinese Americans in primary care. American Journal of Public Health 2010;100(12):2397-402.

Zatzick 2001 \{published data only\}

* Zatzick DF, Roy-Byrne P, Russo JE, Rivara FP, Koike A, Jurkovich GJ, et al.Collaborative interventions for physically injured trauma survivors: A pilot randomized effectiveness trial. General Hospital Psychiatry 2001;23:114-23.

Zatzick 2004 \{published data only\}

* Zatzick D, Roy-Byrne P, Russo J, Rivara F, Droesch R,

Wagner A, et al.A randomized effectiveness trial of stepped collaborative care for acutely injured trauma survivors. Archives of General Psychiatry 2004;61(5):498-506. Zatzick DF. Early PTSD and risk behavior intervention after admission for acute surgical trauma. Proceedings of the 158th Annual Meeting of the American Psychiatric Association; 2005 May 21-26; Atlanta (GA). 2005:No. 23C.

\section{References to studies excluded from this review}

\section{Akerblad 2003 \{published and unpublished data\}}

Akerblad AC, Bengtsson F, Ekselius L, Von Knorring L. Effects of an educational compliance enhancement programme and therapeutic drug monitoring on treatment adherence in depressed patients managed by general practitioners. International Clinical Psychopharmacology 2003; 18:347-54.

\section{Al-Saffar 2005 \{published data only\}}

Al-Saffar N, Deshmukh AA, Carter P, Adib SM. Effect of information leaflets and counselling on antidepressant adherence: Open randomised controlled trial in a psychiatric hospital in Kuwait. International Journal of Pharmacy Practice 2005;13:123-31.

Bolton 2001 \{published data only (unpublished sought but not used)\} Bolton PG, Fergusson KJ, Parker SM, Orman JD. Randomised controlled trial of cognitive-behavioural therapy and routine GP care for major depression. Medical Journal of Australia 2001;175:118-9.

Braamse 2010 \{published data only\}

Braamse AM, van Meijel B, Visser O, van Oppen P, Boenink AD, Eeltink C, et al.Distress and quality of life after autologous stem cell transplantation: a randomized clinical trial to evaluate the outcome of a web-based stepped care intervention. BMC Cancer 2010;10:361.

Britian 1999 \{published data only (unpublished sought but not used)\} Britian O. Integration of services for elderly people reduced costs and use of health services [commentary on Bernabei R, Landi F, Gambassi G, Sgadari A, Zuccala G, Mor V, et al. Randomized trial of impact of model of integrated care and case management for older people living in the community. BMJ 1998;316(7141):1348-51]. Evidence-Based Nursing 1999;2:20.

Brook 2003 \{published data only\} Brook O, van Hout H, Nieuwenhuyse H, Heerdink E. Impact of coaching by community pharmacists on drug attitude of depressive primary care patients and acceptability to patients; a randomized controlled trial. European

Neuropsychopharmacology 2003;13:1-9.

Callahan 1994 \{published data only\}

Callahan CM, Hendrie HC, Dittus RS, Brater DC, Hui SL, Tierney WM. Improving treatment of late-life depression in primary care: A randomized clinical trial. Journal of the American Geriatrics Society 1994;42(8):839-46.

Callahan 2006 \{published data only\}

Callahan CM, Boustani MA, Unverzagt FW, Austrom MG, Damush TM, Perkins AJ, et al.Effectiveness of collaborative care for older adults with Alzheimer disease in primary care: a randomized controlled trial. JAMA 2006;295(18): 2148-57.

Cheok 2003 \{published data only\} Cheok F, Schrader G, Banham D, Marker J, Hordacre AL. Identification, course, and treatment of depression after admission for a cardiac condition: rationale and patient characteristics for the Identifying Depression As a Comorbid Condition (IDACC) project. American Heart Journal 2003;146(6):978-84.

Coleman 1999 \{published data only\}

Coleman EA, Grothaus LC, Sandhu N, Wagner EH. Chronic care clinics: a randomized controlled trial of a new model of primary care for frail older adults. Journal of the American Geriatrics Society 1999;47(7):908-9.

Dobscha 2006 \{published data only\} Dobscha SK, Corson K, Hickam DH, Perrin NA, Kraemer DF, Gerrity MS. Depression decision support in primary care: a cluster randomized trial. Annals of Internal Medicine 2006;145(7):477-87.

Dobscha 2008 \{published data only\}

Dobscha SK, Corson K, Leibowitz RQ, Sullivan MD, Gerrity MS. Rationale, design, and baseline findings from a randomized trial of collaborative care for chronic musculoskeletal pain in primary care. Pain Medicine 2008;9 (8):1050-64.

Dobscha 2009 \{published data only\} Dobscha SK, Corson K, Perrin NA, Hanson GC, Leibowitz $\mathrm{RQ}$, Doak MN, et al.Collaborative care for chronic pain in primary care: a cluster randomized trial. JAMA 2009;301 (12):1242-52.

Dozeman 2007 \{published data only\} Dozeman E, van Schaik DJ, Beekman AT, Stalman WA, Bosmans JE, van Marwijk HW. Depression and anxiety, an indicated prevention (DIP) protocol in homes for the elderly: feasibility and (cost) effectiveness of a stepped care programme. BMC Geriatrics 2007;7:6.

Gellis 2010 \{published and unpublished data\} Gellis ZD, Bruce ML. Problem-solving therapy for subthreshold depression in home healthcare patients with cardiovascular disease. The American Journal of Geriatric Psychiatry 2010;18:464-74.

Geron 2006 \{published and unpublished data\} Geron SM, Keefe B. Moving evidence-based interventions to populations: a case study using social workers in primary 
care. Home Health Care Services Quarterly 2006;25(1-2): 95-113.

Hees 2010 \{published data only\}

Hees HL, Koeter MW, de Vries G, Ooteman W, Schene AH. Effectiveness of adjuvant occupational therapy in employees with depression: design of a randomized controlled trial. BMC Public Health 2010;10:558.

Katon 1992 \{published data only\}

Katon W, Von Korff M, Lin E, Bush T, Russo J, Lipscomb $\mathrm{P}$, et al.A randomized trial of psychiatric consultation with distressed high utilizers. General Hospital Psychiatry 1992; 14(2):86-98.

Kroenke 2009 \{published data only\}

Kroenke K, Bair MJ, Damush TM, Wu J, Hoke S, Sutherland J, et al.Optimized antidepressant therapy and pain self-management in primary care patients with depression and musculoskeletal pain: A randomized controlled trial. JAMA 2009;301(20):2099-110.

Lyles 2003 \{published data only\}

Lyles JS, Hodges A, Collins C, Lein C, Given CW, Given B, et al.Using nurse practitioners to implement an intervention in primary care for high-utilizing patients with medically unexplained symptoms. General Hospital Psychiatry 2003; 25(2):63-73.

Meglic 2010 \{published data only\}

Meglic M, Furlan M, Kuzmanic M, Kozel D, Baraga D, Kuhar I, et al.Feasibility of an eHealth service to support collaborative depression care: results of a pilot study. Journal of Medical Internet Research 2010;12(5):e63.

Mudge 2011 \{published data only\}

Mudge AM, Denaro CP, Scott AC, Atherton JJ, Meyers DE, Marwick TH, et al.Exercise training in recently hospitalized heart failure patients enrolled in a disease management programme: Design of the EJECTION-HF randomized controlled trial. European Journal of Heart Failure 2011;13 (12):1370-5.

Oslin 2004 \{published data only\} Oslin DW, Thompson R, Kallan MJ, TenHave T, Blow FC, Bastani R, et al.Treatment effects from UPBEAT: a randomized trial of care management for behavioral health problems in hospitalized elderly patients. Journal of Geriatric Psychiatry and Neurology 2004;17(2):99-106.

Peveler 1999 \{published data only\}

Peveler R, George C, Kinmonth AL, Campbell M, Thompson C. Effect of antidepressant drug counselling and information leaflets on adherence to drug treatment in primary care: randomised controlled trial. BMJ 1999;319 (7210):612-5.

Pols 2008 \{published and unpublished data\} Pols R, Battersby MW. Coordinated care in the management of patients with unexplained physical symptoms: depression is a key issue. Medical Journal of Australia 2008;188:S133S137.

Raue 2009 \{published data only\}

Raue PJ, Schulberg HC, Heo M, Klimstra S, Bruce ML. Patients' depression treatment preferences and initiation, adherence, and outcome: A randomized primary care study. Psychiatric Services 2009;60(3):337-43.

Rickles 2005 \{published data only\}

Rickles NM, Svarstad BL, Statz-Paynter JL, Taylor LV, Kobak KA. Pharmacist telemonitoring of antidepressant use: effects on pharmacist-patient collaboration. Journal of the American Pharmacists Association 2005;45(3):344-53.

Riegel 2006 \{published data only\}

Riegel B, Carlson B, Glaser D, Romero T. Randomized controlled trial of telephone case management in Hispanics of Mexican origin with heart failure. Journal of Cardiac Failure 2006;12(3):211-9.

\section{Seekles 2009 \{published data only\}}

Seekles W, van Straten A, Beekman A, van Marwijk H, Cuijpers P. Stepped care for depression and anxiety: from primary care to specialized mental health care: a randomised controlled trial testing the effectiveness of a stepped care program among primary care patients with mood or anxiety disorders. BMC Health Services Research 2009;9:90.

\section{Simon 2006 \{published data only\}}

Simon GE, Ludman EJ, Operskalski BH. Randomized trial of a telephone care management program for outpatients starting antidepressant treatment. Psychiatric Services 2006; 57(10):1441-5.

Sirey 2010 \{published data only\}

Sirey JA, Bruce ML, Kales HC. Improving antidepressant adherence and depression outcomes in primary care: The Treatment Initiation and Participation (TIP) Program. The American Journal of Geriatric Psychiatry 2010;18(6):554-62.

Stevens 2009 \{published data only\}

Stevens J, Klima J, Chisolm D, Kelleher K J. A trial of telephone services to increase adolescent utilization of health care for psychosocial problems. Journal of Adolescent Health 2009; 45:564-70.

Van't Veer-Tazelaar 2009 \{published data only\} van't Veer-Tazelaar PJ, van Marwijk HW, van Oppen P, van Hout HP, van der Horst HE, Cuijpers P, et al.Steppedcare prevention of anxiety and depression in late life: A randomized controlled trial. Archives of General Psychiatry 2009;66(3):297-304.

van der Feltz-Cornelis 2006 \{published data only\} van der Feltz-Cornelis CM, van Oppen P, Ader HJ, van Dyck R. Randomised controlled trial of a collaborative care model with psychiatric consultation for persistent medically unexplained symptoms in general practice. Psychotherapy and Psychosomatics 2006;75(5):282-9.

van der Feltz-Cornelis 2010 \{published data only\} van der Feltz-Cornelis CM, Hoedeman R, de Jong FJ, Meeuwissen JAC, Drewes HW, van der Laan NC, et al.Faster return to work after psychiatric consultation for sicklisted employees with common mental health disorders compared to care as usual. A randomized clinical trial. Neuropsychiatric Disease and Treatment 2010;6:375-85.

Wang 2007 \{published data only\}

Wang PS, Simon GE, Avorn J, Azocar F, Ludman EJ, McCulloch J, et al.Telephone screening, outreach, and care 
management for depressed workers and impact on clinical and work productivity outcomes: a randomized controlled trial. JAMA 2007;298(12):1401-11.

Whooley 2000 \{published data only\}

Whooley MA, Stone B, Soghikian K. Randomized trial of case-finding for depression in elderly primary care patients. Journal of General Internal Medicine 2000;15(5):293-300.

\section{References to studies awaiting assessment}

\section{Aragones 2007 \{published data only\}}

* Aragones E, Caballero A, Pinol JL, Lopez-Cortacans G, Badia W, Hernandez JM, et al.Assessment of an enhanced program for depression management in primary care: a cluster randomized controlled trial. The INDI project (Interventions for Depression Improvement). BMC Public Health 2007;7:253.

Aragones E, Lopez-Cortacans G, Badia W, Hernandez JM, Caballero A, Labad A. Improving the role of nursing in the treatment of depression in primary care in Spain. Perspectives in Psychiatric Care 2008;44(4):248-58.

Joubert 2006 \{published data only\}

* Joubert J, Reid C, Joubert L, Barton D, Ruth D, Jackson $\mathrm{D}$, et al.Risk factor management and depression post-stroke: The value of an integrated model of care. Journal of Clinical Neuroscience 2006;13(1):84-90.

Joubert 2008 \{published data only\}

* Joubert J, Joubert L, Reid C, Barton D, Cumming T, Mitchell P, et al.The positive effect of integrated care on depressive symptoms in stroke survivors. Cerebrovascular Diseases 2008;26(2):199-205.

Mareev 2010 \{published data only\}

* Mareev V, Danielyan M, Yu B. Congestive heart failure: A multidisciplinary non-pharmacological approach for changing in re-hospitalisation and prognosis in patients with concomitant anxiety and depression (CHANCE-AND). Journal of Cardiac Failure. Proceedings of the 14th Annual Scientific Meeting, Heart Failure Society of America; 2010 Sept 12-15; San Diego (CA). 2010; Vol. 16:913.

O'Connor 2001 \{published data only\}

* O'Connor A. Evaluation of GP based mood disorder clinic: A randomised controlled trial of an innovative model of treating depressed people in the community. National Research Register 2001.

Walker 2009a \{published data only\}

* Walker J, Cassidy J, Sharpe M, SMaRT Oncology-2

Trialists. The second symptom Management Research Trial in Oncology (SMaRT Oncology-2): A randomised trial to determine the effectiveness and cost-effectiveness of adding a complex intervention for major depressive disorder to usual care for cancer patients. Trials 2009;10:18.

Walker J, Sharpe M. Depression care for people with cancer: a collaborative care intervention. General Hospital Psychiatry 2009;31(5):436-41.

Walker 2009b \{published data only\}

* Walker J, Cassidy J, Sharpe M. The third symptom management research trial in oncology (SMaRT Oncology-
3): A randomised trial to determine the efficacy of adding a complex intervention for major depressive disorder (depression care for people with lung cancer) to usual care, compared to usual care alone in patients with lung cancer. Trials 2009;10:92.

Walker M, Walker L, Walker A, Bateman J, Braid F, Hebblewhite C, et al.Preventing psychiatric morbidity in people with cancer. Psycho-Oncology. Proceedings of the 11th World Congress of Psycho-Oncology of the International Psycho-Oncology Society; 2009 June 21-25; Vienna, Austria. 2009.

Wu 2010 \{published data only\}

* Wu HS, Xu Y, Xu YF, Zhu HX, Zhu LP, Xue JJ, et al.Effectiveness of case management of chronic disease for elderly depression in community [Chinese]. Chinese Mental Health Journal 2010;24(4):245-9.

\section{References to ongoing studies}

\section{Chen 2011 \{published data only\}}

* Chen S, Conwell Y, Xu B, Chiu H, Tu X, Ma Y.

Depression care management for late-life depression in China in primary care: protocol for a randomized controlled trial. Trials 2011;12:121.

Chung 2010 \{published data only\} Chung B. Community partners in care: a partnership to improve depression care in Los Angeles. Proceedings of the 164th Annual General Meeting of the American Psychiatric Association. 2011:333-4.

* Chung B, Jones L, Dixon EL, Miranda J, Wells K, Community Partners in Care Steering Council. Using a community partnered participatory research approach to implement a randomized controlled trial: planning community partners in care. Journal of Health Care for the Poor and Underserved 2010;21(3):780-95.

Cooper 2010 \{published data only\} * Cooper LA, Ford DE, Ghods BK, Roter DL, Primm AB, Larson SM, et al.A cluster randomized trial of standard quality improvement versus patient-centered interventions to enhance depression care for African Americans in the primary care setting: study protocol. Implementation Science 2010;5:18.

Freund 2011 \{published data only\}

* Freund T, Peters-Klimm F, Rochon J, Mahler C, Gensichen J, Erler A, et al.Primary care practice-based care management for chronically ill patients (PraCMan): Study protocol for a cluster randomized controlled trial. Trials 2011;12:163.

Gitlin 2012 \{published data only\}

* Gitlin LN, Harris LF, McCoy M, Chernett NL, Jutkowitz E, Pizzi LT, Beat the Blues Team. A community-integrated home based depression intervention for older African Americans: description of the Beat the Blues randomized trial and intervention costs. BMC Geriatrics 2012;12:4. Jutkowitz E, Pizzi LT, Gitlin L, Foley K, Chernett N, McCoy $\mathrm{M}$. Costs of a community support program for depression: Results from the beat the blues (BTB) trial. Value in Health. 
Proceedings of the 13th Annual International Meeting of the International Society for Pharmacoeconomics and Outcomes Research, ISPOR; 2010 Nov 6-9; Prague Czech Republic. 2010; Vol. 13, issue 7:A449.

Pizzi LT, Jutkowitz E, Gitlin L, Suh DC, Dennis M. Baseline results from beat the blues. Value in Health. Proceedings of the 16th Annual International Meeting of the International Society for Pharmacoeconomics and Outcomes Research, ISPOR; 2011 May 21-25; Baltimore (MD). 2011; Vol. 14: A195.

Graven 2011 \{published data only\}

* Graven C, Brock K, Hill K, Ames D, Cotton S, Joubert L. From rehabilitation to recovery: Protocol for a randomised controlled trial evaluating a goal-based intervention to reduce depression and facilitate participation post-stroke. BMC Neurology 2011;11:73.

Horn 2007 \{published data only\}

* Horn EK, Van Benthem TB, Hakkaart-Van Roijen L, Van Marwijk HWJ, Beekman ATF, Rutten FF, et al.Costeffectiveness of collaborative care for chronically ill patients with comorbid depressive disorder in the general hospital setting, a randomised controlled trial. BMC Health Services Research 2007;7:28.

Ijff 2007 \{published data only\}

Hakkaart L, Van Steenbergen K, Schawo S, Huijbregts K, De Jong $\mathrm{S}$, Van Marwijk H, et al.The cost effectiveness of collaborative care in depressed patients in the primary care. Journal of Mental Health Policy and Economics. Proceedings of the 10th Workshop on Costs and Assessment in Psychiatry; 2011 Mar 25-27; Venice, Italy. 2011. Huijbregts KML, De Jong FJ, Martens F, Adr HJ, Van Marwijk HWJ, Beekman ATF, et al.Effectiveness of collaborative care for depression in Dutch primary care. Journal of Psychosomatic Research. Proceedings of the 13th Annual Meeting of the European Association for Consultation-Liaison Psychiatry and Psychosomatics, EACLPP and the 28th European Conference on Psychosomatic Research, ECPR; 2010 Jun 30-Jul 3; Innsbruck, Austria. 2010.

* Ijff MA, Huijbregts KM, van Marwijk HW, Beekman AT, Hakkaart-van Roijen L, Rutten FF, et al.Cost-effectiveness of collaborative care including PST and an antidepressant treatment algorithm for the treatment of major depressive disorder in primary care; a randomised clinical trial. $B M C$ Health Services Research 2007;7:34.

\section{Mitchell 2011 \{published data only\}}

* Mitchell N, Hewitt C, Adamson J, Parrott S, Torgerson D, Ekers D, et al.A randomised evaluation of CollAborative care and active surveillance for Screen-Positive EldeRs with sub-threshold depression (CASPER): study protocol for a randomized controlled trial. Trials 2012;12:225.

Morgan 2009 \{published data only\}

* Morgan M, Dunbar J, Reddy P, Coates M, Leahy R.

The trueblue study: Is practice nurse-led collaborative care effective in the management of depression for patients with heart disease or diabetes?. BMC Family Practice 2009;10:46.
Muntingh 2009 \{published data only\}

Goorden M, Muntingh A, Balkom AJ, Van Marwijk HW, Spinhoven P, De Waal MW, et al.Cost effectiveness of a collaborative care stepped intervention for anxiety disorders in the primary care setting. Value in Health. Proceedings of the ISPOR 14th Annual European Congress; 2011 Nov 58; Madrid, Spain. 2011.

* Muntingh AD, van der Feltz-Cornelis CM, van Marwijk HW, Spinhoven P, Assendelft WJ, de Waal MW, et al.Collaborative stepped care for anxiety disorders in primary care: Aims and design of a randomized controlled trial. BMC Health Services Research 2009;9:159.

Musselman 2006 \{published data only\}

* Musselman B L. Depression-Diabetes mechanisms: Urban African Americans. www.clinicaltrials.gov/ct2/show/ NCT00209170 (accessed 7 May 2012).

\section{Pommer $2012\{$ published data only\}}

* Pommer AM, Pouwer F, Denollet J, Pop VJM. Managing co-morbid depression and anxiety in primary care patients with asthma and/or chronic obstructive pulmonary disease: study protocol for a randomized controlled trial. Trials 2012;13:6.

Pouwer $\mathrm{CH}$. Disease management of depression and anxiety (DiMaCoDeA) in primary care patients with diabetes mellitus, asthma and/or COPD [NTR2626]. www.trialregister.nl/trialreg/admin/rctview.asp?TC=2626 (accessed 3 May 2012).

Rollman 2004 \{published data only\}

* Rollman BL. The RELAX Trial: Reducing limitations from anxiety in primary care [Telephone-based care management program for individuals with anxiety disorders]. www.clinicaltrials.gov/ct2/show/NCT00158327 (accessed 3 May 2012).

\section{Steel 2011 \{published data only\}}

* Steel J, Geller DA, Tsung A, Marsh JW, Dew MA, Spring $\mathrm{M}$, et al.Randomized controlled trial of a collaborative care intervention to manage cancer-related symptoms: Lessons learned. Clinical Trials 2011;8(3):298-310.

Stoop 2011 \{published data only\}

* Stoop CH, Spek VRM, Pop VJM, Pouwer F. Disease management for co-morbid depression and anxiety in diabetes mellitus: design of a randomised controlled trial in primary care. BMC Family Practice 2011;12:139.

Trinh 2011 \{published data only\}

* Trinh NH, Bedoya CA, Chang TE, Flaherty K, Fava $\mathrm{M}$, Yeung A. A study of a culturally focused psychiatric consultation service for Asian American and Latino American primary care patients with depression. BMC Psychiatry 2011;11:166.

\section{Tylee 2011 \{published data only\}}

Tylee A, Ashworth M, Barley E, Brown J, Chambers J, Farmer A, et al.Up-beat UK: a programme of research into the relationship between coronary heart disease and depression in primary care patients. BMC Family Practice 2011;12:38. 
Yeung 2011 \{published data only\}

* Yeung A, Hails K, Chang T, Trinh NH, Fava M. A study of the effectiveness of telepsychiatry-based culturally sensitive collaborative treatment of depressed Chinese Americans. BMC Psychiatry 2011;11:154.

\section{Zatzick 2011 \{published data only\}}

* Zatzick D, Rivara F, Jurkovich G, Russo J, Trusz SG, Wang $\mathrm{J}$, et al.Enhancing the population impact of collaborative care interventions: Mixed method development and implementation of stepped care targeting posttraumatic stress disorder and related comorbidities after acute trauma. General Hospital Psychiatry 2011;33(2):123-34.

\section{Additional references}

\section{Adams 2004}

Adams G, Gulliford MC, Ukoumunne OC, Eldridge S, Chinn S, Campbell M. Patterns of intra-cluster correlation from primary care research to inform study design and analysis. Journal of Clinical Epidemiology 2004;57:784-793.

\section{APA 2000}

American Psychiatric Association. Diagnostic and Statistical Manual of Mental Disorders. Fourth Text Revision. Washington DC: American Psychiatric Association, 2000.

Attkinson 2003

Attkinson C, Zwixk R. The client satisfaction questionnaire: psychometric properties and correlations with service utilisation and psychotherapy outcome. Evaluation and Program Planning 2003;5:233-7.

\section{Badamgarav 2003}

Badamgarav E, Weingarten S, Henning J, Knight K, Hasselblad V, Gano A, et al.Effectiveness of disease management programs in depression: a systematic review. American Journal of Psychiatry 2003;160:2080-90.

Beck 1987

Beck AT, Steer R. Beck Depression Inventory: Manual. San Antonio, TX: Psychological Corporation, 1987.

\section{Beck 1988}

Beck AT, Epstein N, Brown G, Steer RA. An inventory for measuring clinical anxiety: Psychometric properties. Journal of Consulting and Clinical Psychology 1988;56:893-7.

Bijl 2004

Bijl D, van Marwijk W, de Haan M, van Tilburg W, Beekman A. Effectiveness of disease management programmes for recognition, diagnosis and treatment of depression in primary care: a review. European Journal of General Practice 2004;10:6-12.

\section{Boerma 1999}

Boerma W, Verhaak P. The general practitioner as the first contacted health professional by patients with psychological problems: a European study. Psychological Medicine 1999; 29:689-96.

\section{Bosc 1997}

Bosc M, Dubini A, Poin V. Development and validation of a social functioning scale, the Social Adaption Self-evaluation
Scale. European Neuropsychopharmacology 1997;7 Suppl 1:

S57-S70.

\section{Bower 2005}

Bower P, Gilbody S. Managing common mental health disorders in primary care: conceptual models and evidence base. BMJ 2005;330:839-42.

Bower 2006

Bower P, Gilbody S, Richards D, Fletcher J, Sutton A. Collaborative care for depression in primary care. Making sense of a complex intervention: systematic review and meta regression. British Journal of Psychiatry 2006;189:484-93.

Buszewicz 2011

Buszewicz M, Griffin M, Beecham J, Bonin E, Hutson M. ProCEED Report of a Study of Proactive Care by Practice Nurses for People with Depression and Anxiety. London: MIND, 2011.

Cape 2010

Cape J, Whittington C, Buszewicz M, Wallace P, Underwood L. Effectiveness of brief psychological therapies for anxiety and depression in primary care: meta-analysis and meta-regression. BMC Health Services Research 2010;8: 38.

\section{Churchill 2002}

Churchill R, Hunot V, Corney R, Knapp M, McGuire H, Tylee A, et al.A systematic review of controlled trials of the effectiveness and cost-effectiveness of brief psychological treatments for depression. Health Technology Assessment 2002;5(35):1-173.

\section{Deeks 2011}

Deeks JJ, Higgins JPT, Altman DG (editors). Chapter 9: Analysing data and undertaking meta-analyses. In: Higgins JPT, Green S (editors). Cochrane Handbook for Systematic Reviews of Interventions Version 5.1.0 [updated March 2011]. The Cochrane Collaboration, 2011. Available from www.cochrane-handbook.org.

\section{Donner 2002}

Donner A, Klar N. Issues in the meta-analysis of cluster randomised trials. Statistics in Medicine 2002;21:2971-80.

\section{Egger 1997}

Egger M, Davey-Smith G, Schneider M, Minder C. Bias in meta-analysis detected by a simple graphical test. BMJ 1997;315:629-35.

Gilbody 2003a

Gilbody S, Whitty P, Grimshaw J, Thomas R. Educational and organisational interventions to improve the management of depression in primary care: a systematic review. JAMA 2003;289:3145-51.

Gilbody 2006

Gilbody S, Bower P, Fletcher J, Richards D, Sutton A. Collaborative care for depression: a systematic review and cumulative meta-analysis. Archives of Internal Medicine 2006;166:2314-21.

Gunn 2006

Gunn J, Diggens J, Hegarty K, Blashki G. A systematic review of complex system interventions designed to increase 
recovery from depression in primary care. BMC Health Services Research 2006;6:88.

Higgins 2003

Higgins JP, Thompson SG, Deeks JJ, Altman DG. Measuring inconsistency in meta-analyses. BMJ 2003;327 (7414):557-60.

\section{Higgins 2004}

Higgins JPT, Thompson SG. Controlling the risk of spurious findings from meta-regression. Statistics in Medicine 2004;23:1663-82.

\section{Higgins 2011a}

Higgins JPT, Green S (editors). Cochrane Handbook for Systematic Reviews of Interventions Version 5.1.0 [updated March 2011]. The Cochrane Collaboration, 2011. Available from www.cochrane-handbook.org.

\section{Higgins 2011b}

Higgins JPT, Altman DG, Sterne JAC (editors). Chapter 8: Assessing risk of bias in included studies. In: Higgins JPT, Green S (editors). Cochrane Handbook for Systematic Reviews of Interventions Version 5.1.0 [updated March 2011]. The Cochrane Collaboration, 2011. Available from www.cochrane-handbook.org.

Kates 2007

Kates N, Mach M. Chronic disease management for depression in primary care: a summary of the current literature and implications for practice. Canadian Journal of Psychiatry 2007;52(2):77-85.

Katon 1997

Katon W, Von Korff M, Lin E, Unutzer J, Simon G, Walker $\mathrm{E}$, et al.Population-based care of depression: Effective disease management strategies to decrease prevalence. General Hospital Psychiatry 1997;19(3):169-78.

\section{Kraemer 2002}

Kraemer H, Wilson G, Fairburn C, Agras W. Mediators and moderators of treatment effects in randomized clinical trials. Archives of General Psychiatry 2002;59:877-83.

Kroenke 2001

Kroenke K, Spitzer RL, Williams JBW. The PHQ-9: validity of a brief depression severity measure. Journal of General Internal Medicine 2001;16:606-13.

\section{Kupfer 1991}

Kupfer DJ. Long-term treatment of depression. Journal of Clinical Psychiatry 1991;52 Suppl 5:28-34.

\section{Lingam 2002}

Lingam R, Scott J. Treatment non-adherence in affective disorders. Acta Psychiatrica Scandinavica 2002;105:164-72.

Lipsey 1990

Lipsey M. Design Sensitivity: Statistical Power for

Experimental Research. Newbury Park: Sage, 1990.

\section{Mathers 2006}

Mathers CD, Loncar D. Projections of global mortality and burden of disease from 2002 to 2030. PLoS Medicine 2006; 3(11):e442.

\section{McManus 2009}

McManus S, Meltzer H, Brugha T, Bebbington P, Jenkins R. Adult Psychiatric Morbidity in England, 2007: results of a household survey. Leeds: The Information Centre for Health and Social Care, 2009.

\section{Medical Research Council 2008}

Medical Research Council (UK). Developing and evaluating complex interventions: new guidance. www.mrc.ac.uk/ Utilities/Documentrecord/index.htm?d=MRC004871 (Accessed August 2012).

\section{Neumeyer-Gromen 2004}

Neumeyer-Gromen A, Lampert T, Stark K, Kallisschnigg G. Disease management programs for depression: a systematic review and meta-analysis of randomized controlled trials. Medical Care 2004;42(12):1211-21.

\section{NHS 2002}

NHS Centre for Reviews and Dissemination. Improving the recognition and management of depression in primary care. Effective Health Care Bulletin YR:2002 Vol. 7:1-11.

\section{NICE 2009}

National Institute for Clinical Excellence (NICE). Depression in Adults with a Chronic Physical Health Problem: Treatment and Management - NICE guidance (Clinical guidelines CG91). London: NICE, 2009.

\section{NICE 2010}

National Institute for Clinical Excellence (NICE). NICE

Guidance on the Treatment and Management of Depression in Adults - NICE guidance (Clinical guidelines CG90). London: NICE, 2010

\section{NICE 2011a}

National Institute for Clinical Excellence (NICE). Common Mental Health Disorders: Identification and Pathways to Care - NICE guidance (Clinical guidelines CG123). London: NICE, 2011.

\section{NICE 2011b}

National Institute for Clinical Excellence (NICE). NICE Guidance on Generalised Anxiety Disorder and Panic disorder (with or without agoraphobia) in Adults - NICE guidance (Clinical guidelines CG113). London: NICE, 2011.

Ormel 1999

Ormel J, Von Korff M, Oldehinkel T. Onset of disability in depressed and non-depressed primary care patients. Psychological Medicine 1999;29:847-53.

\section{Roth 1996}

Roth A, Fonagy P. What Works for Whom? A Critical Review of Psychotherapy Research. London: Guildford, 1996.

\section{Sartorius 2001}

Sartorius N. The economic and social burden of depression. Journal of Clinical Psychiatry 2001;62 (Suppl 15):8-11.

\section{Thompson 2002}

Thompson SG, Higgins JP. How should meta-regression analyses be undertaken and interpreted?. Statistics in Medicine 2002;21:1559-73. 
Thota 2012

Thota A, Sipe T, Byard G, Zometa C, Hahn R, McKnightEily $\mathrm{L}$, et al.Collaborative care to improve the management of depressive disorders: A community guide systematic review and meta-analysis. American Journal of Preventive Medicine 2012;42:525.

Ukoumunne 1999

Ukoumunne O, Gulliford M, Chinn S, Sterne J, Burney P. Methods for evaluating area-wide and organisation-based interventions in health and health care: a systematic review. Health Technology Assessment 1999;3(5):iii-92.

\section{Ustun 2004}

Ustun T, Ayuso-Mateos J, Chatterji S, Mathers C, Murray C. Global burden of depressive disorders in the year 2000. British Journal of Psychiatry 2005;184:386-92.

Von Korff 2001

Von Korff M, Goldberg D. Improving outcomes in depression: the whole process of care needs to be enhanced. BMJ 2001;323(7319):948-9.

\section{Wagner 1996}

Wagner E, Austin B, Von Korff M. Organizing care for patients with chronic illness. Milbank Quarterly 1996; Vol.
74:11-43.

Ware 1993

Ware JE, Snow KK, Kosinski M, Gandek B. SF-36 Health Survey: Manual and 1993 Interpretation Guide. Boston, MA: New England Medical Center, Health Institute, 1993.

\section{WHO 1992}

World Health Organization. The ICD-10 Classification of Mental and Behavioural Disorders. Geneva: WHO, 1992.

\section{WHO 2001a}

World Health Organization. Mental and Neurological

Disorder - Factsheet 265. Geneva: WHO, 2001.

\section{WHO 2001b}

World Health Organization. ATLAS - Mental Health

Resources in the World 2001. Geneva: WHO, 2001.

\section{References to other published versions of this review}

Archer 2007

Archer J, Bower P, Gilbody S, Lovell K, Richards D, Gask L, et al.Collaborative care for depression and anxiety problems. Cochrane Database of Systematic Reviews 2007, Issue 2. [DOI: 10.1002/14651858.CD006525]

* Indicates the major publication for the study 


\section{CHARACTERISTICS OF STUDIES}

\section{Characteristics of included studies [ordered by study ID]}

\section{Adler 2004}

\begin{tabular}{ll}
\hline Methods & Study design: Randomised controlled trial \\
\hline Participants & Setting: Primary care \\
& Diagnosis: Met DSM-IV criteria for MDD and/or dysthymia. Established DSM-IV \\
criteria using PC-SADC self-administered & Inclusion criteria: Received care from a PCP, 18 years or older, able to read and understand \\
& English, no acute life threatening condition with a terminal prognosis of 6 months, not \\
pregnant/not given birth within the past 6 months & Exclusion criteria: Current alcoholism (defined as more than one positive response on \\
the CAGE, plus one item assessing current usage), bipolar disorder, and/or psychotic & disorders \\
Age: Mean 42.3 years \\
Gender: $72 \%$ female \\
Ethnicity: $72 \%$ white \\
Country: United States \\
Sample size (randomised): Total participants 533, intervention 268, control 265
\end{tabular}

Interventions

Treatment: Pharmacist intervention

Contains the four elements of collaborative care:

1) a multi-professional approach to patient care: PCP, pharmacist (CM), psychiatrist (MH specialist)

2) a structured management plan: The pharmacist intervention protocol was based on AHCPR guidelines and emphasised: 1) obtaining medication history, 2) assessing side effects or drug interactions, 3) monitoring drug efficacy and toxicity, 4) educating patients about depression and $\mathrm{ADs}, 5)$ encouraging patients to start and maintain $\mathrm{AD}$ therapy, and 6) facilitating communication with PCP

3) scheduled patient follow-ups: Medication = nine times over 18 months (2, 4, 6, 8, 12 weeks, and 6, 9, 12, and 18 months)

4) enhanced inter-professional communication: CM facilitated communication with a patient's PCP, MH specialist provided clinical supervision as needed

Control: Treatment as usual enhanced as PCPs received results of depression screen indicating a DSM-IV diagnosis of major depressive disorder and/or dysthymia

Outcomes

Depression (mBDI): 3, 6, 12, 18 months

Medication use: 3, 6 months

Quality of Life (mental and physical health): 6 months

Notes

AD: antidepressant; CM: case manager; DSM-IV: Diagnostic and Statistical Manual fourth edition; MDD: major depressive disorder; $\mathrm{MH}$ : mental health; PCP: primary care provider; AHCPR: Agency for Health Care Policy and Research; PC-Sad: Primary Care Screener for Affective Disorders; mBDI: modified Beck Depression Inventory

Risk of bias 
Adler 2004 (Continued)

\begin{tabular}{|c|c|c|}
\hline Bias & Authors' judgement & Support for judgement \\
\hline $\begin{array}{l}\text { Random sequence generation (selection } \\
\text { bias) }\end{array}$ & Low risk & Computerised coin-flip \\
\hline Allocation concealment (selection bias) & Unclear risk & Insufficient information available to assess \\
\hline $\begin{array}{l}\text { Incomplete outcome data (attrition bias) } \\
\text { All outcomes }\end{array}$ & Unclear risk & $\begin{array}{l}\text { Short-term loss to follow-up based on pri- } \\
\text { mary depression outcome (mBDI) was: } \\
\text { overall } 149 / 533(28 \%), 75 / 268(28 \%) \text { in- } \\
\text { tervention and } 74 / 265(28 \%) \text { control. Rea- } \\
\text { sons for loss to follow-up not provided at } \\
6 \text { months. Intention-to-treat analysis re- } \\
\text { ported, conducted an extensive analysis of } \\
\text { the potential bias introduced by missing } \\
\text { data using available data }\end{array}$ \\
\hline Selective reporting (reporting bias) & Unclear risk & Insufficient information available to assess \\
\hline Other bias & Unclear risk & Insufficient information available to assess \\
\hline Implementation Integrity & Unclear risk & Insufficient information available to assess \\
\hline $\begin{array}{l}\text { Blinding of participants and personnel } \\
\text { (performance bias) } \\
\text { All outcomes }\end{array}$ & High risk & $\begin{array}{l}\text { Participants and personnel could not be } \\
\text { blinded, outcome likely to be influenced by } \\
\text { lack of blinding }\end{array}$ \\
\hline $\begin{array}{l}\text { Blinding of outcome assessment (detection } \\
\text { bias) } \\
\text { All outcomes }\end{array}$ & Unclear risk & Insufficient information available to assess \\
\hline
\end{tabular}

Araya 2003

Methods

Participants
Study design: Randomised controlled trial

Setting: Primary care

Diagnosis: Current DSM-IV major depression. A score of 5 or more on the general health questionnaire (GHQ-12) at 2 screenings, mini international neuropsychiatric interview (MINI) to ascertain a DSM-IV diagnosis

Inclusion criteria: Low-income, female, primary care patients aged 18 to 70 years

Exclusion criteria: Current psychotic symptoms, serious suicidal risk, history of mania, or current alcohol abuse, psychiatric consultation or admission to hospital in the 3 months before the interview

Age: Mean 42.6 (SD 13.6) years

Gender: $100 \%$ female

Ethnicity: Not stated

Country: Chile 


Treatment: Stepped care
Contains the four elements of collaborative care:
1) a multi-professional approach to patient care: PCP, nurse or social worker group
leaders $(\mathrm{CM})$, psychiatrist $(\mathrm{MH}$ specialist)
2) a structured management plan: Step 1: those scoring 19 or less on HRSD received
psychoeducational group and those scoring $>19$ received psychoeducational group plus
assessment for ADs. Step 2: after 6 week reassessment those scoring 12 or less received
two booster sessions at weeks 9 and 12 and those scoring $>12$ were referred for PCP
reassessment to initiate ADs or adjust ADs. Psychoeducation topics included a manual
with information on symptoms and causes of depression, treatment options, scheduling
positive activities, problem-solving techniques, and basic cognitive and relapse-preven-
tion techniques. PCPs delivered a brief structured pharmacotherapy protocol using a
standard medication algorithm to ensure adequate dose and duration of treatment (flu-
oxetine, amitriptyline, or imipramine). Group leaders monitored medication adherence
and attendance at follow-up visits for patients receiving pharmacotherapy
3) scheduled patient follow-ups: The CM psychoeducational intervention group con-
sisted of seven weekly sessions and two booster sessions at weeks 9 and 12 . CM monitored
AD adherence and attendance at follow-up visits for patients receiving pharmacotherapy
4) enhanced inter-professional communication: CM co-ordinated further management
with PCPs if needed and usually communicated with doctors through alert notes and
arranging appointments for patients, MH specialist provided clinical supervision
Control: Treatment as usual plus prior to the start of the study PCPs in the control group
received guidelines on how to treat depression in primary care

Outcomes

Notes
Depression (HRSD): 3, 6 months

Medication use: 6 months

Quality of Life (mental and physical health): 3, 6 months

CM: case manager; DSM-IV: Diagnostic and Statistical Manual fourth edition; HRSD: Hamilton Rating Scale for Depression; $\mathrm{MH}$ : mental health; PCP: primary care provider; SD: standard deviation

\section{Risk of bias}

\begin{tabular}{lll} 
Bias & Authors' judgement & Support for judgement \\
\hline $\begin{array}{l}\text { Random sequence generation (selection } \\
\text { bias) }\end{array}$ & Low risk & $\begin{array}{l}\text { Computer generated random numbers in } \\
\text { blocks of } 20\end{array}$ \\
\hline
\end{tabular}

Allocation concealment (selection bias) Low risk

Incomplete outcome data (attrition bias) Unclear risk All outcomes
Standard block size. Sealed numbered envelopes opened by an individual not involved in patient recruitment

Short-term loss to follow-up based on primary depression outcome (HDRS < 50\%) was: overall 29/240 (12\%), 16/120 (13\%) intervention and $13 / 120$ (11\%) control. 


\begin{tabular}{|c|c|c|}
\hline & & $\begin{array}{l}\text { Reasons for loss to follow-up not provided. } \\
\text { Used intention-to-treat analysis }\end{array}$ \\
\hline Selective reporting (reporting bias) & Unclear risk & Insufficient information available to assess \\
\hline Other bias & Unclear risk & Insufficient information available to assess \\
\hline Implementation Integrity & Unclear risk & Insufficient information available to assess \\
\hline $\begin{array}{l}\text { Blinding of participants and personnel } \\
\text { (performance bias) } \\
\text { All outcomes }\end{array}$ & High risk & $\begin{array}{l}\text { Participants and personnel could not be } \\
\text { blinded, outcome likely to be influenced by } \\
\text { lack of blinding }\end{array}$ \\
\hline $\begin{array}{l}\text { Blinding of outcome assessment (detection } \\
\text { bias) } \\
\text { All outcomes }\end{array}$ & Low risk & $\begin{array}{l}\text { Assessor was not aware of treatment alloca- } \\
\text { tion }\end{array}$ \\
\hline
\end{tabular}

Asarnow 2005

\begin{tabular}{ll}
\hline Methods & Study design: Randomised controlled trial \\
\hline Participants & Setting: Primary care \\
Diagnosis: Either of 2 criteria: (1) endorsed "stem items" for major depression or dys- \\
thymia from the 12-month Composite International Diagnostic Interview (CIDI-12 \\
[Core version 2.1]) modified slightly to conform to diagnostic criteria for adolescents, \\
1 week or more of past month depressive symptoms, and a total CES-D score of 16 or \\
greater (range of possible scores, 0-60); or (2) a CES-D score of 24 or greater \\
Inclusion criteria: Aged 13 to 21 years and presenting at clinic for primary care visit \\
Exclusion criteria: Having previously completed screening, not English-speaking, clini- \\
cian not in the study, and sibling already in the study \\
Age: Mean 17.2 (SD 2.1) years \\
Gender: $78 \%$ female \\
Ethnicity: $56 \%$ Hispanic/Latino \\
Country: United States \\
Sample size (randomised): Total participants 418, intervention 211, control 207 \\
\hline
\end{tabular}

2) a structured management plan: (1) expert leader teams at each site adapted and implemented the intervention; (2) CMs supported PCPs with patient evaluation, education, medication and psychosocial treatment, and linkage with specialty $\mathrm{MH}$ services; (3) trained CMs delivered manualised CBT; and (4) patient and clinician choice of treatments (CBT, medication, combined CBT and medication, care manager followup, or referral). The CBT manual included a session introducing the treatment model, three 4-session modules emphasising different CBT components (activities/social skills, cognition, and communication/problem solving), and a final session emphasising relapse 


\section{Risk of bias}

\begin{tabular}{|c|c|c|}
\hline Bias & Authors' judgement & Support for judgement \\
\hline $\begin{array}{l}\text { Random sequence generation (selection } \\
\text { bias) }\end{array}$ & Low risk & Computer generated \\
\hline Allocation concealment (selection bias) & Low risk & $\begin{array}{l}\text { Allocation conducted by an individual not } \\
\text { involved in patient recruitment after a time } \\
\text { delay (median, } 21 \text { days) }\end{array}$ \\
\hline $\begin{array}{l}\text { Incomplete outcome data (attrition bias) } \\
\text { All outcomes }\end{array}$ & Low risk & $\begin{array}{l}\text { Short-term loss to follow-up based on pri- } \\
\text { mary depression outcome (CES-D) was: } \\
\text { overall } 74 / 418(18 \%), 41 / 211 \text { (19\%) in- } \\
\text { tervention and } 33 / 207(16 \%) \text { control. Rea- } \\
\text { sons for loss to follow-up provided, with } \\
\text { similar reasons for missing data across } \\
\text { groups. Used intention-to-treat analysis }\end{array}$ \\
\hline Selective reporting (reporting bias) & Unclear risk & Insufficient information available to assess \\
\hline Other bias & Unclear risk & Insufficient information available to assess \\
\hline Implementation Integrity & Unclear risk & Insufficient information available to assess \\
\hline
\end{tabular}


Blinding of participants and personnel High risk (performance bias)

All outcomes
Participants and personnel could not be blinded, outcome likely to be influenced by lack of blinding

Blinding of outcome assessment (detection Low risk

bias)

Assessor was not aware of treatment allocation

All outcomes

Bartels 2004

Methods

Participants
Study design: Randomised controlled trial

Setting: Speciality settings and primary care

Diagnosis: Significant psychological distress on the General Health Questionnaire, a positive response to suicidal ideation questions modified from the PRIME-MD, or atrisk alcohol consumption based on quantity/frequency criteria of more than seven drinks/ week or more than two binge episodes in the past 3 months consisting of more than three drinks on a single occasion. Assessed by using the Mini-International Neuropsychiatric Interview, CES-D scale, Beck Anxiety Inventory, an alcohol quantity/frequency scale, and a detailed medication review

Inclusion criteria: Met criteria for target conditions (depression, anxiety, and at-risk drinking) assessed by using the Mini International Neuropsychiatric Interview, CESD scale, Beck Anxiety Inventory, an alcohol quantity/frequency scale, and a detailed medication review

Exclusion criteria: Patients who had received mental health/substance abuse treatment in the preceding 3 months and patients with severe cognitive impairment ( $\geq 16$ on the Brief Orientation Memory Concentration Test). Primary care providers were given the opportunity to withdraw patients with positive screens for medical reasons; patients with a positive assessment on the Mini International Neuropsychiatric Interview for psychosis, mania, or hypomania, patients with incomplete data

Age: Mean 73.5 (SD 6.2) years

Gender: $26 \%$ female

Ethnicity: $52 \%$ white

Country: United States

Sample size (randomised): Total participants 2022, intervention 999, control 1023

Intervention: Integrated care

Contains the four elements of collaborative care:

1) a multi-professional approach to patient care: PCP, social workers, psychologists, psychiatric nurses, psychiatrists, and counsellors (CM)

2) a structured management plan: Integrated models included: 1) mental health and substance abuse services co-located in the primary care setting (including counselling, case management, psychotherapy, and pharmacological treatment); 2) services provided by licensed mental health/substance abuse providers (including social workers, psychologists, psychiatric nurses, psychiatrists, and counsellors); 3) verbal or written communication between the MH specialist and PCP; and 4) an appointment within 2 to 4 weeks following the PCP visit. Specific clinical interventions were not required. Patients with at-risk drinking were offered a manualised Brief Alcohol Intervention 
Bartels 2004 (Continued)

3) scheduled patient follow-ups: Alcohol $=3$ counselling sessions, depression $=$ the intervention varied across sites

4) enhanced inter-professional communication: Verbal or written communication about the clinical evaluation and treatment plan between the CMs and PCP. PCPs were required to remain involved in the patients' care and document their role in the medical record, and communicate with the CMs. PCPs were notified in the event that a patient failed to attend the initial clinical visit

Control: Enhanced referral model which provided mental health/substance abuse services in a specialty setting that was physically separate and designated as a mental health/ substance abuse clinic. The minimum criteria for the enhanced referral model included 1 ) referral within 2-4 weeks of the PCP appointment; 2) comply with model requirements, including time to first appointment and coordinated follow-up contacts if the patient failed to make the first scheduled visit; 3) assistance with transportation; and 4) assistance in meeting the costs of treatment

Outcomes

Depression (CES-D): 3, 6 months

Quality of Life (mental and mental health): 3, 6 months

Satisfaction: 3 months

Notes

CES-D: Centre for Epidemiological Studies Depression; CM: case manager; $\mathrm{MH}$ : mental health; PCP: primary care provider; SD: standard deviation

\section{Risk of bias}

\begin{tabular}{|c|c|c|}
\hline Bias & Authors' judgement & Support for judgement \\
\hline $\begin{array}{l}\text { Random sequence generation (selection } \\
\text { bias) }\end{array}$ & High risk & $\begin{array}{l}\text { Computer generated blocks, the computer } \\
\text { system malfunctioned for } 2 \text { weeks and } 44 \\
\text { patients did not conform to randomisation } \\
\text { procedure. Some patients assigned using } \\
\text { social security number (even or odd) }\end{array}$ \\
\hline
\end{tabular}

Allocation concealment (selection bias) Low risk

Incomplete outcome data (attrition bias) Unclear risk All outcomes
Researchers telephoned an independent person to receive patient allocation

Short-term loss to follow-up based on primary depression outcome (CES-D) was: overall $311 / 1531(20 \%), 159 / 758(21 \%)$ intervention and 152/773 (20\%) control. Reasons for loss to follow-up not provided. Used intention-to-treat analysis

\begin{tabular}{lll}
\hline Selective reporting (reporting bias) & Low risk & $\begin{array}{l}\text { Protocol available and all prespecified out- } \\
\text { comes reported }\end{array}$ \\
\hline Other bias & Unclear risk & Insufficient information available to assess \\
\hline Implementation Integrity & Unclear risk & Insufficient information available to assess
\end{tabular}


Bartels 2004 (Continued)

Blinding of participants and personnel High risk (performance bias)

All outcomes

Blinding of outcome assessment (detection High risk

bias)

All outcomes
Participants and personnel could not be blinded, outcome likely to be influenced by lack of blinding

Assessor was potentially aware of treatment allocation

\section{Blanchard 1995}

\begin{tabular}{|c|c|}
\hline Methods & Study design: Randomised controlled trial \\
\hline Participants & $\begin{array}{l}\text { Setting: Community and primary care } \\
\text { Diagnosis: Depression measured by short-CARE. Short-CARE contains a diagnostic } \\
\text { depression scale (DPDS) which identifies subjects who are likely to be suffering from } \\
\text { pervasive depression, a level of depression warranting clinical interventions } \\
\text { Inclusion criteria: Older adults } \\
\text { Exclusion criteria: Not stated } \\
\text { Age: Mean } 76.3 \text { years } \\
\text { Gender: } 86 \% \text { female } \\
\text { Ethnicity: Not stated } \\
\text { Country: United Kingdom } \\
\text { Sample size (randomised): Total participants } 96 \text {, intervention } 47 \text {, control } 49\end{array}$ \\
\hline Interventions & $\begin{array}{l}\text { Intervention: Community nurse management } \\
\text { Contains the four elements of collaborative care: } \\
\text { 1) a multi-professional approach to patient care: General practitioner (PCP), community } \\
\text { psychiatric nurse (CM), old age psychiatry team (MH specialist) } \\
\text { 2) a structured management plan: Initial assessment by psychiatrist and information } \\
\text { then presented to MH specialist team and individually-tailored care plans developed and } \\
\text { implemented by CM. Interventions were negotiated with the patient and their PCP. In- } \\
\text { terventions included: medication trial and review, increasing social network, counselling } \\
\text { when specific interpersonal/bereavement problems were identified, behaviour therapy } \\
\text { and review of physical health } \\
\text { 3) scheduled patient follow-ups: } 12 \text { weekly face-to-face sessions } \\
\text { 4) enhanced inter-professional communication: CM worked in close liaison with PCP } \\
\text { who remained clinically responsible. Interventions were negotiated with the PCP. The } \\
\text { CM was in regular contact with the MH specialist and could use them at any time in a } \\
\text { consultative capacity } \\
\text { Control: Initial assessment with psychiatrist and case then presented to MH specialist } \\
\text { (old age psychiatry team) and an individual management plan was developed which was } \\
\text { shared with the PCP after the intervention period. PCPs were made aware of severity of } \\
\text { depressive symptoms of patients }\end{array}$ \\
\hline
\end{tabular}

Outcomes

Notes
Depression (short CARE): 3 months

Medication use: 3 months

CM: case manager; $\mathrm{MH}$ : mental health; PCP: primary care provider 
Blanchard 1995 (Continued)

\section{Risk of bias}

\begin{tabular}{|c|c|c|}
\hline Bias & Authors' judgement & Support for judgement \\
\hline $\begin{array}{l}\text { Random sequence generation (selection } \\
\text { bias) }\end{array}$ & Unclear risk & Insufficient information available to assess \\
\hline Allocation concealment (selection bias) & Unclear risk & Insufficient information available to assess \\
\hline $\begin{array}{l}\text { Incomplete outcome data (attrition bias) } \\
\text { All outcomes }\end{array}$ & Unclear risk & $\begin{array}{l}\text { Short-term loss to follow-up based on pri- } \\
\text { mary depression outcome (Short CARE } \\
\text { DPDS) was: overall } 14 / 96 \text { (15\%), } 4 / 47 \\
(9 \%) \text { intervention and 10/49 (20\%) con- } \\
\text { trol. Reasons for loss to follow-up provided, } \\
\text { with similar reasons for missing data across } \\
\text { groups. Intention-to-treat analysis not re- } \\
\text { ported, no description of methods for man- } \\
\text { aging missing data }\end{array}$ \\
\hline Selective reporting (reporting bias) & Unclear risk & Insufficient information available to assess \\
\hline Other bias & Unclear risk & Insufficient information available to assess \\
\hline Implementation Integrity & Unclear risk & Insufficient information available to assess \\
\hline $\begin{array}{l}\text { Blinding of participants and personnel } \\
\text { (performance bias) } \\
\text { All outcomes }\end{array}$ & High risk & $\begin{array}{l}\text { Participants and personnel could not be } \\
\text { blinded, outcome likely to be influenced by } \\
\text { lack of blinding }\end{array}$ \\
\hline $\begin{array}{l}\text { Blinding of outcome assessment (detection } \\
\text { bias) } \\
\text { All outcomes }\end{array}$ & Low risk & $\begin{array}{l}\text { Assessor was not aware of treatment alloca- } \\
\text { tion }\end{array}$ \\
\hline
\end{tabular}

Bogner 2008

Methods

Participants
Study design: Pilot randomised controlled trial

Setting: Primary care

Diagnosis: A diagnosis of depression or a prescription for an antidepressant medication within the past year

Inclusion criteria: Aged 50 years and older, a systolic blood pressure of $140 \mathrm{~mm} \mathrm{Hg}$ or greater or diastolic blood pressure of $90 \mathrm{~mm} \mathrm{Hg}$ or greater for non-diabetic patients, or a systolic blood pressure of $130 \mathrm{~mm} \mathrm{Hg}$ or greater or a diastolic blood pressure of 80 $\mathrm{mm} \mathrm{Hg}$ or greater for patients with diabetes on at least 2 visits in the previous year, or a prescription for an antihypertensive medication within the past year

Exclusion criteria: Cognitive impairment, unable to communicate in English, residing in a care facility that provides medications on a schedule, unable to use Medication Event 
Bogner 2008 (Continued)

Monitoring System (MEMS) caps (AARDEX, Zug, Switzerland), which are microelectronic monitoring devices

Age: Mean 58.6 (SD 6.8) years

Gender: $77 \%$ female

Ethnicity: 83\% African American

Country: Unitd States

Sample size (randomised): Total participants 64, intervention 32, control 32

Interventions

Intervention: Integrated care

Contains the four elements of collaborative care:

1) a multi-professional approach to patient care: Family physician (PCP), research coordinator (CM), academic PCP (MH specialist)

2) a structured management plan: Intervention focused on depression and hypertension and aimed to promote patients' adherence to antihypertensive and AD treatment. CM collaborated with physicians to help patients understand and recognise depression in the context of hypertension, offered patients guideline-based treatment recommendations, monitored treatment adherence and clinical status, assessed for side-effects and assistance in their management, and provided appropriate follow-up or referral. Individualised programme congruent with patients' social and cultural context

3) scheduled patient follow-ups: 3 face-to-face, 2 phone contacts in 4-week period

4) enhanced inter-professional communication: CM acted as liaison between the PCP and patient to help patients recognise depression in the context of hypertension. CM received weekly supervision from $\mathrm{MH}$ specialist

Control: Treatment as usual

Outcomes Depression (CES-D): 2, 4, 6, 12 weeks

Medication use: 2, 4, 6 weeks

Notes

AD: antidepressant; CES-D: Centre for Epidemiological Studies Depression; CM: case manager; $\mathrm{MH}$ : mental health; PCP: primary care provider; SD: standard deviation

Risk of bias

\begin{tabular}{|c|c|c|}
\hline Bias & Authors' judgement & Support for judgement \\
\hline $\begin{array}{l}\text { Random sequence generation (selection } \\
\text { bias) }\end{array}$ & Unclear risk & Insufficient information available to assess \\
\hline Allocation concealment (selection bias) & Unclear risk & Insufficient information available to assess \\
\hline $\begin{array}{l}\text { Incomplete outcome data (attrition bias) } \\
\text { All outcomes }\end{array}$ & Low risk & $\begin{array}{l}\text { Short-term loss to follow-up based on pri- } \\
\text { mary depression outcome (CES-D) was: } \\
\text { overall } 0 / 64(0 \%), 0 / 32(0 \%) \text { intervention } \\
\text { and } 0 / 32(0 \%) \text { control } \\
\text { Intention-to-treat analysis not reported }\end{array}$ \\
\hline Selective reporting (reporting bias) & Unclear risk & Insufficient information available to assess \\
\hline Other bias & Unclear risk & Insufficient information available to assess \\
\hline
\end{tabular}

Collaborative care for depression and anxiety problems (Review)

Copyright $\odot 2012$ The Cochrane Collaboration. Published by John Wiley \& Sons, Ltd. 
Bogner 2008 (Continued)

\begin{tabular}{|l|l|l}
\hline Implementation Integrity & Low risk & $\begin{array}{l}\text { Attempts were made to assess implemen- } \\
\text { tation integrity (e.g. direct observation or } \\
\text { rating of tapes) }\end{array}$
\end{tabular}

Blinding of participants and personnel High risk (performance bias)

Participants and personnel could not be

All outcomes blinded, outcome likely to be influenced by lack of blinding

Blinding of outcome assessment (detection Unclear risk bias)

All outcomes

Bogner 2010

Methods

Participants

Interventions
Study design: Pilot randomised controlled trial

Setting: Primary Care

Diagnosis: A diagnosis of depression or a prescription for an antidepressant within the past year

Inclusion criteria: Diabetics aged 50 and older, an $\mathrm{A} 1 \mathrm{C}>7$ at their last primary care office visit or a prescription for an oral hypoglycaemic agent within the past year, older African Americans prescribed pharmacotherapy for type 2 diabetes mellitus and depression from physicians at a large primary care practice

Exclusion criteria: Not stated

Age: Mean 60 years

Gender: $85 \%$ female

Ethnicity: 100\% African American

Country: United States

Sample size (randomised): Total participants 58, intervention 29, control 29

Intervention: Integrated care

Contains the four elements of collaborative care:

1) a multi-professional approach to patient care: Family physician (PCP), research coordinator (CM), academic PCP (MH specialist)

2) a structured management plan: Intervention focused on depression in the context of type 2 diabetes mellitus and aimed to promote patients' adherence to an oral hypoglycaemic agent and AD. CM collaborated with PCP to help participants understand and recognise depression in the context of type 2 diabetes mellitus, offered guidelinebased treatment recommendations, monitored adherence and clinical status, assessment for the presence of side effects and assistance in their management, and provided appropriate follow-up or referral. Individualised programme congruent with patients' social and cultural context

3) scheduled patient follow-ups: 3 face-to-face, 2 phone contacts in 4-week period

4) enhanced inter-professional communication: CM acted as liaison between PCP and the elderly depressed patient with type 2 diabetes mellitus in promoting adherence to medication. CM received weekly supervision from specialist

Control: Treatment as usual 
Bogner 2010 (Continued)

\begin{tabular}{|c|c|c|}
\hline Outcomes & \multicolumn{2}{|c|}{$\begin{array}{l}\text { Depression (CES-D): } 12 \text { weeks } \\
\text { Medication use: } 2,4,6,12 \text { weeks }\end{array}$} \\
\hline Notes & \multicolumn{2}{|c|}{$\begin{array}{l}\text { AD: antidepressant; CES-D: Centre for Epidemiological Studies Depression; CM: case } \\
\text { manager; } \mathrm{MH} \text { : mental health; PCP: primary care provider }\end{array}$} \\
\hline \multicolumn{3}{|l|}{ Risk of bias } \\
\hline Bias & Authors' judgement & Support for judgement \\
\hline $\begin{array}{l}\text { Random sequence generation (selection } \\
\text { bias) }\end{array}$ & Unclear risk & Insufficient information available to assess \\
\hline Allocation concealment (selection bias) & Unclear risk & Insufficient information available to assess \\
\hline $\begin{array}{l}\text { Incomplete outcome data (attrition bias) } \\
\text { All outcomes }\end{array}$ & Low risk & $\begin{array}{l}\text { Short-term loss to follow-up based on pri- } \\
\text { mary depression outcome (CES-D) was: } \\
\text { overall } 0 / 58(0 \%), 0 / 29(0 \%) \text { intervention } \\
\text { and } 0 / 29(0 \%) \text { control. Intention-to-treat } \\
\text { analysis not reported }\end{array}$ \\
\hline Selective reporting (reporting bias) & Unclear risk & Insufficient information available to assess \\
\hline Other bias & Unclear risk & Insufficient information available to assess \\
\hline Implementation Integrity & Low risk & $\begin{array}{l}\text { Attempts were made to assess implemen- } \\
\text { tation integrity (e.g. direct observation or } \\
\text { rating of tapes) }\end{array}$ \\
\hline $\begin{array}{l}\text { Blinding of participants and personnel } \\
\text { (performance bias) } \\
\text { All outcomes }\end{array}$ & High risk & $\begin{array}{l}\text { Participants and personnel could not be } \\
\text { blinded, outcome likely to be influenced by } \\
\text { lack of blinding }\end{array}$ \\
\hline $\begin{array}{l}\text { Blinding of outcome assessment (detection } \\
\text { bias) } \\
\text { All outcomes }\end{array}$ & Unclear risk & Insufficient information available to assess \\
\hline
\end{tabular}

Bruce 2004

\begin{tabular}{|c|c|}
\hline Methods & Study design: Cluster-randomised controlled trial \\
\hline Participants & $\begin{array}{l}\text { Setting: Primary care } \\
\text { Diagnosis: All patients with a CES-D score higher than } 20 \text { as well as a } 5 \% \text { random sample } \\
\text { of patients with lower scores. Patients scoring } 20 \text { or lower and not selected randomly were } \\
\text { recruited if they responded positively to supplemental questions about prior depressive } \\
\text { episodes or treatment } \\
\text { Inclusion criteria: Age } 60 \text { years or older, ability to give informed consent, Mini-Mental } \\
\text { State Examination (MMSE) score of } 18 \text { or higher, and ability to communicate in English }\end{array}$ \\
\hline
\end{tabular}


Bruce 2004 (Continued)

Exclusion criteria: Not stated
Age: Range 60 to 94 years
Gender: $72 \%$ female
Ethnicity: $67 \%$ white
Country: United States
Sample size (randomised): Total clusters 20, intervention 10, control 10; Total partici-
pants 598 , intervention 320, control 278

Interventions

Intervention: Primary care intervention

Contains the four elements of collaborative care:

1) a multi-professional approach to patient care: PCP, social workers, nurses, and psychologists (CM), psychiatrist (MH specialist)

2) a structured management plan: The Prevention of Suicide in Primary Care Elderly: Collaborative Trial (PROSPECT) intervention had two major components (1) PCP worked with a clinical algorithm for treating geriatric depression in a primary care setting which recommended a first-line trial of a SSRI (citalopram), (2) When a patient declined medication therapy, the PCP could recommend IPT from the CM. CMs monitored depressive symptoms, medication adverse effects, and treatment adherence

3) scheduled patient follow-ups: CM interacted with patients at scheduled intervals or when clinically necessary

4) enhanced inter-professional communication: Practice based CMs collaborated regularly with PCPs and received weekly supervision from MH specialist and additional monthly IPT supervision

Usual care: Treatment as usual enhanced by educating PCPs about the treatment guidelines and notifying them when a patient met criteria for depression diagnosis

Outcomes

Depression (BDI-II): 3, 6, 12, 18, 24 months

Medication use: 24 months

Quality of Life (mental and physical health): 24 months

Notes

BDI: Beck Depression Inventory; CES-D: Centre for Epidemiological Studies Depression; CM: case manager; IPT: interpersonal psychotherapy treatment; $\mathrm{MH}$ : mental health; PCP: primary care provider; SSRI: selective serotonin reuptake inhibitor

\section{Risk of bias}

\begin{tabular}{|c|c|c|}
\hline Bias & Authors' judgement & Support for judgement \\
\hline $\begin{array}{l}\text { Random sequence generation (selection } \\
\text { bias) }\end{array}$ & Low risk & Coin-toss \\
\hline Allocation concealment (selection bias) & Unclear risk & Insufficient information available to assess \\
\hline $\begin{array}{l}\text { Incomplete outcome data (attrition bias) } \\
\text { All outcomes }\end{array}$ & Unclear risk & $\begin{array}{l}\text { Short-term loss to follow-up based on pri- } \\
\text { mary depression outcome (HRSD) was: } \\
\text { overall } 122 / 598(20 \%), 72 / 320(23 \%) \text { in- } \\
\text { tervention and } 50 / 278(18 \%) \text { control. Rea- } \\
\text { sons for loss to follow-up not provided. } \\
\text { Used intention-to-treat analysis }\end{array}$ \\
\hline
\end{tabular}

Collaborative care for depression and anxiety problems (Review) 
Bruce 2004 (Continued)

\begin{tabular}{l|l|l}
\hline Selective reporting (reporting bias) & Low risk & $\begin{array}{l}\text { Protocol available and all prespecified out- } \\
\text { comes reported }\end{array}$ \\
\hline Other bias & Unclear risk & Insufficient information available to assess \\
\hline $\begin{array}{l}\text { Implementation Integrity } \\
\begin{array}{l}\text { Blinding of participants and personnel } \\
\text { (performance bias) }\end{array}\end{array}$ & High risk & Insufficient information available to assess \\
\hline $\begin{array}{l}\text { All outcomes } \\
\text { Blinding of outcome assessment (detection } \\
\text { bias) } \\
\text { All outcomes }\end{array}$ & Unclear risk & $\begin{array}{l}\text { Participants and personnel could not be } \\
\text { blinded, outcome likely to be influenced by } \\
\text { lack of blinding }\end{array}$ \\
\hline
\end{tabular}

\section{Buszewicz 2010}

\begin{tabular}{ll} 
Methods & Study design: Randomised controlled trial \\
\hline Participants & Setting: Primary care \\
& Diagnosis: Two or more documented episodes of depression within the previous three \\
& years, evidence of recurrent and/or chronic depression (measured with the Composite \\
& International Diagnostic Interview), a score of 14 indicating mild depression on the \\
& BDI-II \\
& Inclusion criteria: Men and women aged 18 and over, sufficient English to be able to \\
& complete self-report questionnaires \\
& Exclusion criteria: Current psychotic symptoms, impaired cognitive function, incapaci- \\
& tating alcohol or drug dependence \\
& Age: Mean 48.4 years \\
Gender: $75 \%$ female & Ethnicity: $90 \%$ white British \\
Country: United Kingdom & Sample size (randomised): Total participants 558, intervention 282, control 276
\end{tabular}

Interventions

Intervention: Proactive care

Contains the four elements of collaborative care:

1) a multi-professional approach to patient care: General Practitioner (PCP), practice nurse (CM), study team of General Practitioner with Special Interest in $\mathrm{MH}$ and psychologist (MH specialist)

2) a structured management plan: Baseline assessment including current treatment and side-effects, potential treatments (medication, psychological therapies or social interventions) and an educational booklet about depression and its treatment. Social factors were explored (for example social isolation, low physical activity, unemployment, finance, housing) and appropriate advice given or referrals to other agencies made. A collaborative individualised plan was formulated and reviewed during follow-ups, together with clinical review and progress towards goals. Plan also included relapse prevention 3) scheduled patient follow-ups: 10 appointments over a two-year period at baseline, after one month, then two months later and every three months for the remainder of 
Buszewicz 2010 (Continued)

the 24 month period. Reviews could be over telephone

4) enhanced inter-professional communication: If practice based CMs were concerned they discuss patient with the GP. CMs had regular telephone contact (every three to four months) with the $\mathrm{MH}$ specialist

Control: Treatment as usual and it was stipulated that the participants should not see the practice nurse for any $\mathrm{MH}$ intervention, although they might see the nurse for physical health problems

Outcomes

Depression (BDI-II): 3, 6, 12, 18 and 24 months

Antidepressant use: 24 months

Social: 24 months

Quality of Life: 24 months

Other: Primary care and mental health utilisation, informal care, costs

Notes

BDI: Beck Depression Inventory; CM: case manager; $\mathrm{MH}$ : mental health; PCP: primary care provider

\section{Risk of bias}

\begin{tabular}{|c|c|c|}
\hline Bias & Authors' judgement & Support for judgement \\
\hline $\begin{array}{l}\text { Random sequence generation (selection } \\
\text { bias) }\end{array}$ & Low risk & Independent computer service \\
\hline Allocation concealment (selection bias) & Low risk & Central independent allocation \\
\hline $\begin{array}{l}\text { Incomplete outcome data (attrition bias) } \\
\text { All outcomes }\end{array}$ & Unclear risk & $\begin{array}{l}\text { Short-term loss to follow-up based on pri- } \\
\text { mary depression outcome (BDI-II) was: } \\
\text { overall } 190 / 558(34 \%), 81 / 282(29 \%) \text { in- } \\
\text { tervention and } 109 / 276(39 \%) \text { control. } \\
\text { Reasons for loss to follow-up not provided. } \\
\text { Used intention-to-treat analysis, multiple } \\
\text { imputation used to manage missing data }\end{array}$ \\
\hline Selective reporting (reporting bias) & Low risk & $\begin{array}{l}\text { Protocol available and all prespecified out- } \\
\text { comes reported }\end{array}$ \\
\hline Other bias & Unclear risk & Insufficient information available to assess \\
\hline Implementation Integrity & Unclear risk & Insufficient information available to assess \\
\hline $\begin{array}{l}\text { Blinding of participants and personnel } \\
\text { (performance bias) } \\
\text { All outcomes }\end{array}$ & High risk & $\begin{array}{l}\text { Participants and personnel could not be } \\
\text { blinded, outcome likely to be influenced by } \\
\text { lack of blinding }\end{array}$ \\
\hline $\begin{array}{l}\text { Blinding of outcome assessment (detection } \\
\text { bias) } \\
\text { All outcomes }\end{array}$ & Low risk & $\begin{array}{l}\text { Assessor was not aware of treatment alloca- } \\
\text { tion }\end{array}$ \\
\hline
\end{tabular}

Collaborative care for depression and anxiety problems (Review)

Copyright $\Subset 2012$ The Cochrane Collaboration. Published by John Wiley \& Sons, Ltd. 
Methods

Participants
Study design: Randomised controlled trial

\section{Setting: Primary care}

Diagnosis: A new episode of depression and started on an antidepressant medication. Depression assessed using the Primary Care Evaluation of Mental Disorders (PRIMEMD)

Inclusion criteria: Diagnosed with a new episode of depression (PRIME-MD) and started on an antidepressant medication

Exclusion criteria: Age of $<18$ years, terminal illness, psychosis, recent (within the past 3 months) alcohol (AUDIT score of $>8$ ) or substance abuse, two or more suicide attempts, pregnancy or nursing, limited command of the English language, and unwillingness to use the University of Washington Family Medical Centre as a source of care for the next 12 months

Age: Mean 38.7 (SD 13.5) years

Gender: $77 \%$ female

Ethnicity: $78 \%$ white

Country: United States

Sample size (randomised): Total participants 74, intervention 41, control 33
Interventions
Intervention: Enhanced care

Contains the four elements of collaborative care:

1) a multi-professional approach to patient care: PCP, pharmacist (CM), study psychiatrist ( $\mathrm{MH}$ specialist)

2) a structured management plan: CMs addressed depressive symptoms and medicationrelated concerns. The initial contacts focused on support and education, as well as medication dosage adjustment and the management of adverse effects including change or discontinuation of $\mathrm{ADs}$, and provision of additional pharmacotherapy for insomnia or sexual dysfunction, as needed. Appointments with mental health providers were also facilitated

3) scheduled patient follow-ups: 13 contacts during 12 month period. Weekly telephone calls for the first four weeks, followed by phone contact every two weeks through week 12. During months 4-12, the subjects received a telephone call every other month 4) enhanced inter-professional communication: PCPs were informed of medication changes made by $\mathrm{CM}$ and shared computerised medical records used. On a bi-monthly basis, the CM and $\mathrm{MH}$ specialist reviewed individual cases. Patients were referred to PCP and/or psychiatrist if suicidal ideation detected (also to psychiatrist if no symptom improvement)

Control: Treatment as usual enhanced as case managers assessed patients and patients encouraged to use available resources

Outcomes

Depression (HSCL-20): 3, 6, 9, 12 months

Medication use: 3, 6, 9, 12 months

Satisfaction: 3, 6, 9, 12 months

Notes

AD: antidepressant; CM: case manager; HSCL: Hopkins Sympton Checklist; MH: mental health; PCP: primary care provider

\section{Risk of bias}




\section{Capoccia 2004 (Continued)}

\begin{tabular}{|c|c|c|}
\hline Bias & Authors' judgement & Support for judgement \\
\hline $\begin{array}{l}\text { Random sequence generation (selection } \\
\text { bias) }\end{array}$ & Low risk & Computer generated \\
\hline Allocation concealment (selection bias) & Unclear risk & Insufficient information available to assess \\
\hline $\begin{array}{l}\text { Incomplete outcome data (attrition bias) } \\
\text { All outcomes }\end{array}$ & Unclear risk & $\begin{array}{l}\text { Short-term loss to follow-up based on pri- } \\
\text { mary depression outcome (HSCL-20 > } \\
50 \%) \text { was: overall } 4 / 74(5 \%), 2 / 41(5 \%) \text { in- } \\
\text { tervention and } 2 / 33(6 \%) \text { control. Reasons } \\
\text { for loss to follow-up not provided. Used in- } \\
\text { tention-to-treat analysis }\end{array}$ \\
\hline Selective reporting (reporting bias) & Low risk & $\begin{array}{l}\text { Protocol available and all prespecified out- } \\
\text { comes reported }\end{array}$ \\
\hline Other bias & High risk & $\begin{array}{l}\text { Case managers had some contact with pa- } \\
\text { tients in the control group }\end{array}$ \\
\hline Implementation Integrity & Unclear risk & Insufficient information available to assess \\
\hline $\begin{array}{l}\text { Blinding of participants and personnel } \\
\text { (performance bias) } \\
\text { All outcomes }\end{array}$ & High risk & $\begin{array}{l}\text { Participants and personnel could not be } \\
\text { blinded, outcome likely to be influenced by } \\
\text { lack of blinding }\end{array}$ \\
\hline $\begin{array}{l}\text { Blinding of outcome assessment (detection } \\
\text { bias) } \\
\text { All outcomes }\end{array}$ & Low risk & $\begin{array}{l}\text { Assessor was not aware of treatment alloca- } \\
\text { tion }\end{array}$ \\
\hline
\end{tabular}

\section{Chaney 2011}

Methods

Participants
Study design: Cluster-randomised controlled trial

Setting: Primary care

Diagnosis: Probable major depression based on a PHQ-9 score of 10 or above. Patients with subthreshold depression (an initial PHQ-9 between five and nine) who also had a) a prior history of depression, or b) dysthymia were also eligible

Inclusion criteria: At least one primary care appointment in the preceding 12 months in a participating practice, and having one pending appointment scheduled within the three months post-selection

Exclusion criteria: Conditions that required urgent care (acute suicidality, psychosis), inability to communicate over the telephone, or prior naturalistic referral by the patient's PCP to the CM

Age: Mean 64.2 years

Gender: $4 \%$ female

Ethnicity: $87 \%$ white 


\section{Country: United States}

Sample size (randomised): Total clusters 10, intervention 7, control 3; Total participants 761, intervention 386, control 375

Interventions

Intervention: Translating Initiatives for Depression into Effective Solutions (TIDES)

Contains the four elements of collaborative care:

1) a multi-professional approach to patient care: Primary care clinician (PCP), nurse (CM), psychiatrist ( $\mathrm{MH}$ specialist)

2) a structured management plan: PCPs were educated and CMs conducted a telephone assessment and sent the patient education materials. For each patient's treatment plan (i.e. watchful waiting, medication, or referral to $\mathrm{CBT} /$ mental health specialist), the CM provided follow-up, assessed symptom severity, medication adherence and side-effects, as well as relapse prevention

3) scheduled patient follow-ups: Watchful waiting $=1$ call at 3 months, Medication $=5$ telephone calls at 1 or 2 weeks, 1, 2, 3 and 6 months, CBT/MH referral $=2$ telephone calls at 1 or 2 weeks and 6 months

4) enhanced inter-professional communication: CM communicates assessment to PCP who initiates referral or medication. CM completes follow-ups in collaboration with PCP and MH specialist. CM has weekly supervision with $\mathrm{MH}$ specialist

Control: Treatment as usual

$\begin{array}{ll}\text { Outcomes } & \text { Depression (PHQ-9): } 7 \text { months } \\ \text { Medication use: } 7 \text { months } \\ \text { Quality of Life (mental and physical health): } 7 \text { months } \\ \text { Satisfaction: } 7 \text { months }\end{array}$

Notes

CBT: cognitive behaviour therapist; CM: case manager; $\mathrm{MH}$ : mental health; PCP: primary care provider; PHQ-9: Patient Health Questionnaire-9

Risk of bias

\begin{tabular}{l|ll} 
Bias & Authors' judgement & Support for judgement \\
\hline $\begin{array}{l}\text { Random sequence generation (selection } \\
\text { bias) }\end{array}$ & Unclear risk & Insufficient information available to assess \\
\hline Allocation concealment (selection bias) & Unclear risk & Insufficient information available to assess \\
\hline
\end{tabular}

Incomplete outcome data (attrition bias) Unclear risk All outcomes
Short-term loss to follow-up based on primary depression outcome (PHQ-9) was: overall 215/761 (28\%), 98/386 (25\%) intervention and $117 / 375$ (31\%) control. Reasons for loss to follow-up given, similar reasons for missing data across groups. Used intention-to-treat analysis, methods for handling missing data not reported

Selective reporting (reporting bias)
Insufficient information available to assess 


\section{Chaney 2011 (Continued)}

\begin{tabular}{l|l|l}
\hline Other bias & Unclear risk & Insufficient information available to assess \\
\hline Implementation Integrity & Unclear risk & Insufficient information available to assess \\
\hline $\begin{array}{l}\text { Blinding of participants and personnel } \\
\text { (performance bias) } \\
\text { All outcomes }\end{array}$ & High risk & $\begin{array}{l}\text { Participants and personnel could not be } \\
\text { blinded, outcome likely to be influenced by } \\
\text { lack of blinding }\end{array}$ \\
\hline $\begin{array}{l}\text { Blinding of outcome assessment (detection } \\
\text { bias) } \\
\text { All outcomes }\end{array}$ & Unclear risk & Insufficient information available to assess \\
\hline
\end{tabular}

Chew-Graham 2007

Methods

Participants

Interventions
Study design: Randomised controlled trial

Setting: Primary Care

Diagnosis: Clinically identified as depressed. Score of 5 or more on the Geriatric Depression Scale (GDS) and 24 or more on the Mini-Mental State Exam (MMSE)

Inclusion criteria: Over the age of 60

Exclusion criteria: Not stated

Age: Mean 75.5 years

Gender: $72 \%$ female

Ethnicity: Not stated

Country: United Kingdom

Sample size (randomised): Total participants 105, intervention 53, control 52

Intervention: Collaborative care

Contains the four elements of collaborative care:

1) a multi-professional approach to patient care: GP (PCP), community psychiatric nurse (CM), old age psychiatrist ( $\mathrm{MH}$ specialist)

2) a structured management plan: The complex intervention included education about depression, advice about antidepressant medication, a manualised facilitated self-help intervention (SHADE), and sign-posting to other services, particularly voluntary agencies

3) scheduled patient follow-ups: The intervention lasted for 12 weeks and consisted of six face-to-face sessions in each patient's home and five sessions delivered via the telephone 4) enhanced inter-professional communication: CM liaised closely with PCPs and had regular access to advice from $\mathrm{MH}$ specialist according to a defined protocol. The protocol did not define how often the CM liaised with the PCP (by post, email, telephone, or face-to-face) but the CM sent a written report to the PCP at assessment, 4, 8 and 12 weeks. In between, the CM liaised with the PCP in-person if changes in medication were required or if there were concerns about concordance or risk. CM reviewed patients' progress with $\mathrm{MH}$ specialist every 4 weeks or sooner if CM had concerns

Control: Treatment as usual enhanced as all practices were supplied with hand delivered guidelines which outlined diagnostic criteria, suggestions of appropriate investigations, and the primary care management of depression in older people 
Chew-Graham 2007 (Continued)

\begin{tabular}{|c|c|c|}
\hline Outcomes & \multicolumn{2}{|c|}{ Depression (HSCL-20): 4 months } \\
\hline Notes & \multicolumn{2}{|c|}{$\begin{array}{l}\text { CM: case manager; GP: general practitioner; HSCL: Hopkins Sympton Checklist; MH: } \\
\text { mental health; PCP: primary care provider }\end{array}$} \\
\hline \multicolumn{3}{|l|}{ Risk of bias } \\
\hline Bias & Authors' judgement & Support for judgement \\
\hline $\begin{array}{l}\text { Random sequence generation (selection } \\
\text { bias) }\end{array}$ & Low risk & Computer generated \\
\hline Allocation concealment (selection bias) & Low risk & $\begin{array}{l}\text { Allocation conducted by an individual not } \\
\text { involved in patient recruitment }\end{array}$ \\
\hline $\begin{array}{l}\text { Incomplete outcome data (attrition bias) } \\
\text { All outcomes }\end{array}$ & Low risk & $\begin{array}{l}\text { Short-term loss to follow-up based on pri- } \\
\text { mary depression outcome (Proportion with } \\
5+\text { symptoms on SCID) was: overall } 17 / \\
105(16 \%), 8 / 53(15 \%) \text { intervention and } \\
9 / 52(17 \%) \text { control. Reasons for loss to } \\
\text { follow-up provided, with similar reasons } \\
\text { across groups. Used intention-to-treat anal- } \\
\text { ysis }\end{array}$ \\
\hline Selective reporting (reporting bias) & Unclear risk & Insufficient information available to assess \\
\hline Other bias & Unclear risk & Insufficient information available to assess \\
\hline Implementation Integrity & Unclear risk & Insufficient information available to assess \\
\hline $\begin{array}{l}\text { Blinding of participants and personnel } \\
\text { (performance bias) } \\
\text { All outcomes }\end{array}$ & High risk & $\begin{array}{l}\text { Participants and personnel could not be } \\
\text { blinded, outcome likely to be influenced by } \\
\text { lack of blinding }\end{array}$ \\
\hline $\begin{array}{l}\text { Blinding of outcome assessment (detection } \\
\text { bias) } \\
\text { All outcomes }\end{array}$ & Low risk & $\begin{array}{l}\text { Assessor was not aware of treatment alloca- } \\
\text { tion }\end{array}$ \\
\hline
\end{tabular}

Ciechanowski 2004

\begin{tabular}{ll} 
Methods & Study design: Randomised controlled trial \\
\hline Participants & Setting: Community \\
& Diagnosis: DSM-IV minor depression or dysthymia diagnostic criteria. 2-item Primary \\
& Care Evaluation of Mental Disorders (PRIME-MD) depression screening tool and Struc- \\
& tured Clinical Interview for DSM-IV (SCID) as a second-level screen \\
& Inclusion criteria: Aged 60 years or older receiving services from senior service agencies
\end{tabular}


Ciechanowski 2004 (Continued)

or living in senior public housing

Exclusion criteria: No depression, major depression, bipolar disorder, psychosis, substance abuse, cognitive impairment

Age: Mean 73 (SD 8.5) years

Gender: $79 \%$ female

Ethnicity: $42 \%$ ethnic minority

Country: United States

Sample size (randomised): Total participants 138, intervention 72, control 66

Interventions

Intervention: Programme to Encourage Active, Rewarding Lives for Seniors (PEARLS) Contains the four elements of collaborative care:

1) a multi-professional approach to patient care: Primary care physicians (PCPs), social workers (CM), study psychiatrist (MH specialist)

2) a structured management plan: PST sessions were modified to provide greater emphasis on social and physical activation. The goal of physical activation was to assist patients in developing a regular physical activity programme consistent with national recommendations for moderate activity of at least 30 minutes' duration at least 5 days per week. Physical activation began during the third or fourth PST session, allowing patients to develop familiarity with problem-solving skills. The goal of social activation was to increase patients' interactions outside the home by using a resource list under the guidance of the CM. Each session included selecting and engaging in pleasant activities, using a suggestion list if necessary

3) scheduled patient follow-ups: Eight 50-minute in-home sessions over 19 weeks, in weeks $1,2,3,5,7,11,15$, and 19 . After 19 weeks, monthly brief telephone contact to assess clinical progress and use of PST

4) enhanced inter-professional communication: $\mathrm{CM}$ and $\mathrm{MH}$ specialist met weekly or biweekly to review patients. MH specialist contacted PCP for patients lacking progress to recommend initiating or adjusting ADs and to assess potential medical and substance abuse aetiologies for depression. The $\mathrm{MH}$ specialist occasionally clarified details by contacting PCPs

Control: Treatment as usual enhanced by letters sent to PCPs and social workers reporting depression diagnosis with recommendations to continue treatment as usual

Outcomes

Depression (HSCL-20): 6, 12 months

Medication use: 6, 12 months

Quality of Life (mental and physical health): 12 months

Notes

CM: case manager; DSM-IV: Diagnostic and Statistical Manual fourth edition; HSCL: Hopkins Sympton Checklist; MH: mental health; PCP: primary care provider; SD: standard deviation; PST: problem solving therapy

Risk of bias

\begin{tabular}{|c|c|c|}
\hline Bias & Authors' judgement & Support for judgement \\
\hline $\begin{array}{l}\text { Random sequence generation (selection } \\
\text { bias) }\end{array}$ & Unclear risk & $\begin{array}{l}\text { Block randomisation using a } 50: 50 \text { ran- } \\
\text { domisation allocation ratio in blocks of } 10 \\
\text { (changed to } 60: 40 \text { after } 11 \text { blocks) }\end{array}$ \\
\hline
\end{tabular}

Collaborative care for depression and anxiety problems (Review) 


\section{Ciechanowski 2004 (Continued)}

\begin{tabular}{|c|c|c|}
\hline Allocation concealment (selection bias) & Unclear risk & $\begin{array}{l}\text { Standard block size. An individual not in- } \\
\text { volved in patient recruitment created en- } \\
\text { velopes containing concealed assignment } \\
\text { codes assigned sequentially by a research as- } \\
\text { sociate }\end{array}$ \\
\hline $\begin{array}{l}\text { Incomplete outcome data (attrition bias) } \\
\text { All outcomes }\end{array}$ & Low risk & $\begin{array}{l}\text { Short-term loss to follow-up based on pri- } \\
\text { mary depression outcome (HSCL response } \\
\geq 50 \text { decrease) was: overall 6/138 (4\%), 3/ } \\
72(4 \%) \text { intervention and } 3 / 66(5 \%) \text { con- } \\
\text { trol. Reasons for loss to follow-up provided, } \\
\text { with similar reasons across groups. Used in- } \\
\text { tention-to-treat analysis }\end{array}$ \\
\hline Selective reporting (reporting bias) & Unclear risk & Insufficient information available to assess \\
\hline Other bias & Unclear risk & Insufficient information available to assess \\
\hline Implementation Integrity & Low risk & $\begin{array}{l}\text { Attempts were made to assess implemen- } \\
\text { tation integrity (e.g. direct observation or } \\
\text { rating of tapes) }\end{array}$ \\
\hline $\begin{array}{l}\text { Blinding of participants and personnel } \\
\text { (performance bias) } \\
\text { All outcomes }\end{array}$ & High risk & $\begin{array}{l}\text { Participants and personnel could not be } \\
\text { blinded, outcome likely to be influenced by } \\
\text { lack of blinding }\end{array}$ \\
\hline $\begin{array}{l}\text { Blinding of outcome assessment (detection } \\
\text { bias) } \\
\text { All outcomes }\end{array}$ & Unclear risk & Insufficient information available to assess \\
\hline
\end{tabular}

Ciechanowski 2010

\begin{tabular}{ll} 
Methods & Study design: Randomised controlled trial \\
\hline Participants & Setting: Specialist setting \\
& Diagnosis: Clinically significant depression based on a score $\geq 10$ on the PHQ-9 \\
& Inclusion criteria: English reading and speaking, 18 years or older, had an ICD-9 epilepsy \\
& diagnosis, and had attended the UW Regional Epilepsy Centre or neurology clinics \\
& within 2 years of recruitment \\
& Exclusion criteria: Pregnancy or nursing, bipolar or psychotic disorder, current psychiatric \\
& treatment, substance abuse based on the CAGE questionnaire, cognitive impairment \\
& Age: Mean 43.9 (SD 11) years \\
& Gender: $53 \%$ female \\
& Ethnicity: $8 \%$ ethnic minority \\
Country: United States & Sample size (randomised): Total participants 80 , intervention 40 , control 40
\end{tabular}


Ciechanowski 2010 (Continued)

\begin{tabular}{|c|c|}
\hline Interventions & $\begin{array}{l}\text { Intervention: Programme to Encourage Active, Rewarding Lives for Seniors (PEARLS) } \\
\text { Contains the four elements of collaborative care: } \\
\text { 1) a multi-professional approach to patient care: Neurologist (PCPs), social workers } \\
\text { (CM), study psychiatrist (MH specialist) } \\
\text { 2) a structured management plan: PST sessions were modified to provide greater empha- } \\
\text { sis on social and physical activation. The goal of physical activation was to assist patients } \\
\text { in developing a regular physical activity programme consistent with national recommen- } \\
\text { dations for mild to moderate activity of } 30 \text { minutes } 5 \text { days per week that would provide } \\
\text { benefits but not increase risk for inducing seizures. Physical activation began during } \\
\text { the third or fourth PST session, allowing patients to develop familiarity with problem- } \\
\text { solving skills. The goal of social activation was to increase patients' interactions outside } \\
\text { the home by using a resource list under the guidance of the CM. Each session included } \\
\text { selecting and engaging in pleasant activities, using a suggestion list if necessary } \\
\text { 3) scheduled patient follow-ups: Eight } 50 \text {-minute in-home sessions over } 19 \text { weeks, in } \\
\text { weeks } 1,2,3,5,7 \text {, } 11,15 \text {, and } 19 \text {. After } 19 \text { weeks, monthly brief telephone contact to } \\
\text { assess clinical progress and use of PST } \\
\text { 4) enhanced inter-professional communication: CM and MH specialist met weekly or } \\
\text { biweekly to review patients. MH specialist contacted PCP for patients lacking progress } \\
\text { to recommend initiating or adjusting ADs and to assess potential medical and substance } \\
\text { abuse aetiologies for depression. The MH specialist occasionally clarified details by con- } \\
\text { tacting PCPs } \\
\text { Control: Treatment as usual enhanced by letters sent to PCPs and social workers reporting } \\
\text { depression diagnosis with recommendations to continue treatment as usual }\end{array}$ \\
\hline Outcomes & $\begin{array}{l}\text { Depression (HSCL-20): 6, 12, } 18 \text { months } \\
\text { Medication use: } 12 \text { months } \\
\text { Quality of Life (mental and physical health): 6,12, } 18 \text { months }\end{array}$ \\
\hline Notes & $\begin{array}{l}\text { CM: case manager; DSM-IV: Diagnostic and Statistical Manual fourth edition; HSCL: } \\
\text { Hopkins Sympton Checklist; MH: mental health; PCP: primary care provider; PHQ- } \\
\text { 9: Patient Health Questionnaire-9; SD: standard deviation }\end{array}$ \\
\hline
\end{tabular}

Risk of bias

\begin{tabular}{|c|c|c|}
\hline Bias & Authors' judgement & Support for judgement \\
\hline $\begin{array}{l}\text { Random sequence generation (selection } \\
\text { bias) }\end{array}$ & Low risk & $\begin{array}{l}\text { Computer generated in blocks using 50:50 } \\
\text { allocation ratio }\end{array}$ \\
\hline Allocation concealment (selection bias) & Low risk & $\begin{array}{l}\text { An individual not involved in the interven- } \\
\text { tion generated randomisation sequence, } \\
\text { enrolled and allocated patients }\end{array}$ \\
\hline
\end{tabular}

Incomplete outcome data (attrition bias) Low risk All outcomes
Short-term loss to follow-up based on primary depression outcome (HSCL20) was: overall 15/80 (19\%), 8/40 (20\%) intervention and $7 / 40(18 \%)$ control. Reasons for loss to follow-up provided, with similar rea- 
Ciechanowski 2010 (Continued)

sons across groups. Used intention-to-treat analysis

\begin{tabular}{lll}
\hline Selective reporting (reporting bias) & Unclear risk & Insufficient information available to assess \\
\hline Other bias & Unclear risk & Insufficient information available to assess \\
\hline Implementation Integrity & Low risk & $\begin{array}{l}\text { Attempts were made to assess implemen- } \\
\text { tation integrity (e.g. direct observation or } \\
\text { rating of tapes) }\end{array}$ \\
\hline
\end{tabular}

Blinding of participants and personnel High risk

Participants and personnel could not be (performance bias)

blinded, outcome likely to be influenced by All outcomes lack of blinding

Blinding of outcome assessment (detection Low risk bias)

Assessor was not aware of treatment alloca-

All outcomes

Clarke 2005

Methods

Participants
Study design: Randomised controlled trial

Setting: Primary care

Diagnosis: At least one recent dispense of an SSRI antidepressant medication prescribed by a paediatric PCP. Current, research-ascertained DSM episode of major depression Inclusion criteria: Adolescents 12 to 18 years old

Exclusion criteria: Chart indication of schizophrenia or significant developmental/intellectual disability. Extreme suicidal risk that resulted in hospitalisation

Age: Mean 15.3 years

Gender: $78 \%$ female

Ethnicity: 14\% ethnic minority

Country: United States

Sample size (randomised): Total participants 152, intervention 77, control 75
Treatment: Brief CBT plus treatment as usual SSRIs

Contains the four elements of collaborative care:

1) a multi-professional approach to patient care: Paediatric primary care provider (PCP) , psychologist/CBT (CM), psychologist (MH specialist)

2) a structured management plan: All patients were being treated with an SSRI at enrolment. In addition those in the treatment group received brief CBT based on adult and adolescent depression programmes. Acute phase: After initial decision making session (session 1), CBT began with a choice of either four sessions of cognitive restructuring or four sessions of behavioural activation. A workbook was provided. After completion of the first module (sessions 2-5) progress was evaluated and if appropriate the second module commenced (if recovered the youth entered maintenance phase). The second module (sessions 6-9) consisted of the skills training approach not delivered in the first module. The acute phase also aimed to maximise SSRI medication adherence by review- 
Clarke 2005 (Continued)

ing compliance, reported benefits/side effects, and risk of discontinuation. Limited psy-
choeducation about the benefits of SSRI medication and the importance of adherence
was provided
Maintenance phase: CM made brief telephone calls after completing acute sessions
3) scheduled patient follow-ups: Acute phase: $6-9$ sessions of CBT delivered by CM;
maintenance phase: CMs made brief telephone calls to patients $1,2,3,5,7$, and 9
months after completing acute sessions. Also option to request as many as six additional,
in-person sessions during the year long continuation phase
4) enhanced inter-professional communication: On-going communication with PCP
was part of protocol. CMs received weekly supervision from study psychologists
Control: Treatment as usual enhanced as all patients were being treated with an SSRI at
enrolment

\section{Risk of bias}

\begin{tabular}{|c|c|c|}
\hline Bias & Authors' judgement & Support for judgement \\
\hline $\begin{array}{l}\text { Random sequence generation (selection } \\
\text { bias) }\end{array}$ & Unclear risk & $\begin{array}{l}\text { Block randomisation. Insufficient informa- } \\
\text { tion available to assess }\end{array}$ \\
\hline Allocation concealment (selection bias) & Unclear risk & Insufficient information available to assess \\
\hline $\begin{array}{l}\text { Incomplete outcome data (attrition bias) } \\
\text { All outcomes }\end{array}$ & Unclear risk & $\begin{array}{l}\text { Short-term loss to follow-up based on pri- } \\
\text { mary depression outcome (CES-D) was: } \\
\text { overall } 25 / 152(16 \%), 12 / 77(16 \%) \text { inter- } \\
\text { vention and } 13 / 75(17 \%) \text { control. Reasons } \\
\text { for loss to follow-up not provided. Used in- } \\
\text { tention-to-treat analysis }\end{array}$ \\
\hline Selective reporting (reporting bias) & Unclear risk & Insufficient information available to assess \\
\hline Other bias & Unclear risk & Insufficient information available to assess \\
\hline Implementation Integrity & Low risk & $\begin{array}{l}\text { Attempts were made to assess implemen- } \\
\text { tation integrity (e.g. direct observation or } \\
\text { rating of tapes) }\end{array}$ \\
\hline $\begin{array}{l}\text { Blinding of participants and personnel } \\
\text { (performance bias) } \\
\text { All outcomes }\end{array}$ & High risk & $\begin{array}{l}\text { Participants and personnel could not be } \\
\text { blinded, outcome likely to be influenced by } \\
\text { lack of blinding }\end{array}$ \\
\hline
\end{tabular}


Clarke 2005 (Continued)

Blinding of outcome assessment (detection Low risk

Assessor was not aware of treatment allocabias)

tion

All outcomes

Cole 2006

Methods

Study design: Randomised controlled trial

Participants

Setting: Specialist setting and primary care

Diagnosis: Major depression (as defined by DSM-IV criteria) assessed using the Diagnostic Interview Schedule

Inclusion criteria: All patients aged 65 years and over admitted from the emergency department to medical services and scored 4 or less on the Short Portable Mental Status Questionnaire (indicating at most mild cognitive impairment)

Exclusion criteria: Admitted to the intensive care unit or cardiac monitoring unit for more than 48 hours, had an imminently terminal illness, did not speak or understand English or French; and did not live on the Island of Montreal

Age: Mean 78

Gender: $69.4 \%$ female

Ethnicity: Not stated

Country: Canada

Sample size (randomised): Total participants 157, intervention 78, control 79

Interventions

Treatment: Systematic detection and multidisciplinary care

Contains the four elements of collaborative care:

1) a multi-professional approach to patient care: Family physician (PCP), research nurse (CM), psychiatrist ( $\mathrm{MH}$ specialist)

2) a structured management plan: (1) assessment and treatment by $\mathrm{MH}$ specialist in the hospital's geriatric service; (2) follow-up by the CM; and (3) follow-up by the patient's PCP. Treatment involved supportive psychotherapy and drug therapy with an AD, prescribed according to clinical practice guidelines. Contacts involved monitoring condition, providing supportive psychotherapy, ensuring maximum compliance with their treatment and liaising with the family, MH specialist and PCP

3) scheduled patient follow-ups: Pre-discharge: at least weekly. Post-discharge: CM visited or telephoned weekly for 24 weeks

4) enhanced inter-professional communication: $\mathrm{CM}$ liaised with PCP and $\mathrm{MH}$ specialist and updated $\mathrm{MH}$ specialist when patient followed up by PCP. Regular meetings between $\mathrm{CM}$ and $\mathrm{MH}$ specialist to assure consistency in diagnosis and management of depression Control: Treatment as usual enhanced as patients were advised of depression diagnosis and advised to inform PCP

Outcomes

Depression (HAM-D): 6 months

Medication use: 6 months

Quality of Life (mental and physical health): 6 months

Notes

AD: antidepressant; CM: case manager; DSM-IV: Diagnostic and Statistical Manual fourth edition; HAM-D: Hamilton Depression Rating Scale; MH: mental health; PCP: primary care provider 
Cole 2006 (Continued)

\section{Risk of bias}

\begin{tabular}{|c|c|c|}
\hline Bias & Authors' judgement & Support for judgement \\
\hline $\begin{array}{l}\text { Random sequence generation (selection } \\
\text { bias) }\end{array}$ & Unclear risk & $\begin{array}{l}\text { Block randomisation (size varied ran- } \\
\text { domly) using } 1: 1 \text { allocation ratio }\end{array}$ \\
\hline
\end{tabular}

Allocation concealment (selection bias) Low risk

An individual not involved in patient recruitment prepared sealed envelopes allocated in order

Incomplete outcome data (attrition bias) Unclear risk All outcomes

Short-term loss to follow-up based on primary depression outcome $(\geq 50 \%$ decrease in HAMD) was: overall 93/157 (59\%), 45/ 78 (58\%) intervention and 48/79 (61\%) control. Reasons for loss to follow-up provided, with similar reasons for missing data across groups. Intention-to-treat analysis reported based on the assumption data is missing at random

\begin{tabular}{l|l|l}
\hline Selective reporting (reporting bias) & Unclear risk & Insufficient information available to assess \\
\hline Other bias & Unclear risk & Insufficient information available to assess \\
\hline $\begin{array}{l}\text { Implementation Integrity } \\
\text { Blinding of participants and personnel } \\
\begin{array}{l}\text { performance bias) } \\
\text { All outcomes }\end{array}\end{array}$ & High risk & Insufficient information available to assess \\
\hline $\begin{array}{l}\text { Blinding of outcome assessment (detection } \\
\text { bias) } \\
\text { All outcomes }\end{array}$ & Low risk & $\begin{array}{l}\text { Participants and personnel could not be } \\
\text { blinded, outcome likely to be influenced by } \\
\text { lack of blinding }\end{array}$ \\
\hline
\end{tabular}

Datto 2003

\begin{tabular}{ll} 
Methods & Study design: Pilot cluster-randomised controlled trial \\
\hline Participants & Setting: Primary care \\
& Diagnosis: Significant depressive symptoms (Community Epidemiologic Survey of De- \\
pression, CES-D, score at least 16) & Inclusion criteria: Significant depressive symptoms (as above) \\
Exclusion criteria: Significant suicidal risk, ongoing substance abuse problems, current \\
psychotic symptoms, or evidence for bipolar affective disorder \\
Age: Mean 47.6 (SD 16.7) years \\
Gender: $61 \%$ female \\
Ethnicity: $80 \%$ white
\end{tabular}


Datto 2003 (Continued)

Country: United States

Sample size (randomised): Total clusters 35, intervention 17, control 18; Total participants 61, intervention 30, control 31

Interventions

Treatment: Telephone disease management

Contains the four elements of collaborative care:

1) a multi-professional approach to patient care: Primary care physicians (PCP), nurse with $\mathrm{MH}$ experience (CM), psychiatrist ( $\mathrm{MH}$ specialist)

2) a structured management plan: Baseline assessment and then telephone assessments (structured but not scripted) to assess symptoms and gaining information on treatment recommendations and assessing adherence. Prompted by computer generation the CM discussed topics such as depression as a treatable medical illness, treatment options (including psychotherapy and medications), coping skills for stress, risk factors for depression, suicide prevention strategies, and reinforcing follow-up with the PCP. Educational materials on these topics were also sent to the patient. Treatment recommendations made by the CM were general and often referred the PCP back to a particular stage of the depression treatment algorithm, modelled after the AHRQ depression guidelines

3) scheduled patient follow-ups: Following baseline telephone assessment follow up was attempted every 3 weeks during 16 week treatment period

4) enhanced inter-professional communication: After each assessment the results were fed back to the PCP using summary letters, including the scores of the depression measures and a clinical interpretation of them. All PCPs had contact with the $\mathrm{MH}$ specialist as needed. CM had weekly supervision with $\mathrm{MH}$ specialist to facilitate treatment planning and follow-up

Control: Treatment as usual enhanced as included patient evaluation and diagnosis, patient education, provider education and practice guidelines, final outcome and provider feedback

Outcomes

Depression (CES-D): 16 weeks

Notes

CES-D: Community Epidemiologic Survey of Depression; CM: case manager; $\mathrm{MH}$ : mental health; PCP: primary care provider; AHRQ: Agency for Healthcare Research and Quality

\section{Risk of bias}

\begin{tabular}{|c|c|c|}
\hline Bias & Authors' judgement & Support for judgement \\
\hline $\begin{array}{l}\text { Random sequence generation (selection } \\
\text { bias) }\end{array}$ & Unclear risk & Insufficient information available to assess \\
\hline Allocation concealment (selection bias) & Unclear risk & Insufficient information available to assess \\
\hline $\begin{array}{l}\text { Incomplete outcome data (attrition bias) } \\
\text { All outcomes }\end{array}$ & Unclear risk & $\begin{array}{l}\text { Short-term loss to follow-up based on pri- } \\
\text { mary depression outcome (CESD) was: } \\
\text { overall } 11 / 61(18 \%), 5 / 30(17 \%) \text { interven- } \\
\text { tion and } 6 / 31(19 \%) \text { control. Reasons for } \\
\text { loss to follow-up not provided. Intention- } \\
\text { to-treat analysis not reported }\end{array}$ \\
\hline
\end{tabular}


Datto 2003 (Continued)

\begin{tabular}{l|ll}
\hline Selective reporting (reporting bias) & Unclear risk & Insufficient information available to assess \\
\hline Other bias & Unclear risk & Insufficient information available to assess \\
\hline $\begin{array}{l}\text { Implementation Integrity } \\
\begin{array}{l}\text { Blinding of participants and personnel } \\
\text { (performance bias) } \\
\text { All outcomes }\end{array}\end{array}$ & High risk & Insufficient information available to assess \\
\hline $\begin{array}{l}\text { Blinding of outcome assessment (detection } \\
\text { bias) } \\
\text { All outcomes }\end{array}$ & High risk & $\begin{array}{l}\text { Participants and personnel could not be } \\
\text { blinded, outcome likely to be influenced by } \\
\text { lack of blinding }\end{array}$ \\
\hline
\end{tabular}

\section{Dietrich 2004}

Methods

Participants
Study design: Cluster-randomised controlled trial

Setting: Primary care

Diagnosis: Meeting criteria for Diagnostic and Statistical Manual of Mental Disorders fourth edition for major depressive disorder and dysthymia. Diagnosis determined using a structured interview. The severity of depression was assessed with the Hopkins symptom checklist-20, with a score of 0.5 or more required for enrolment

Inclusion criteria: 18 years or older and starting or changing treatment for depression. Participants had to have a telephone and speak English

Exclusion criteria: Being unobtainable for an evaluation interview within 14 days of their index primary care visit, pregnant, suicidal thoughts, schizophrenia, bipolar disorder, post-traumatic stress disorder, or a substance misuse disorder

Age: Mean 42 years

Gender: $80 \%$ female

Ethnicity: $17 \%$ ethnic minority

Country: United States

Sample size (randomised): Total clusters 60, intervention 32, control 28; Total participants 405, intervention 224, control 181

Interventions

Intervention: Quality improvement programme

Contains the four elements of collaborative care:

1) a multi-professional approach to patient care: Primary care clinicians (PCP), primary care or mental health nurses (CM), psychiatrist ( $\mathrm{MH}$ specialist)

2) a structured management plan: A systematic approach to the assessment and management of depression by PCPs which involved CMs assisting patients in overcoming barriers to adherence to the management plan and supporting self-management practices such as exercise or engaging in social activities

3) scheduled patient follow-ups: One week after initial visit and monthly thereafter until remission

4) enhanced inter-professional communication: PCPs received faxed report about patient progress and care management actions after each call. $\mathrm{MH}$ specialists provided suggestions for clinical management to PCPs either via CM faxes or by telephone. PCPs 
Dietrich 2004 (Continued)

can also request advice from $\mathrm{MH}$ specialist at specified times in the week

Control: Treatment as usual enhanced as clinicians took part in a 45-60 minute programme on diagnosis of depression and assessment of suicidal thoughts

\begin{tabular}{ll} 
Outcomes & $\begin{array}{l}\text { Depression (HSCL-20): 3, } 6 \text { months } \\
\text { Medication use: 3,6 months } \\
\text { Satisfaction: 3, 6 months }\end{array}$ \\
\hline Notes & $\begin{array}{l}\text { CM: case manager; HSCL: Hopkins Sympton Checklist; MH: mental health; PCP: } \\
\text { primary care provider }\end{array}$
\end{tabular}

primary care provider

\section{Risk of bias}

\begin{tabular}{|c|c|c|}
\hline Bias & Authors' judgement & Support for judgement \\
\hline $\begin{array}{l}\text { Random sequence generation (selection } \\
\text { bias) }\end{array}$ & Low risk & Coin-toss \\
\hline Allocation concealment (selection bias) & Unclear risk & $\begin{array}{l}\text { Central randomisation of clinic, those re- } \\
\text { cruiting patients may have been aware of } \\
\text { allocation }\end{array}$ \\
\hline $\begin{array}{l}\text { Incomplete outcome data (attrition bias) } \\
\text { All outcomes }\end{array}$ & Unclear risk & $\begin{array}{l}\text { Short-term loss to follow-up based on pri- } \\
\text { mary depression outcome (HSCL-20) was: } \\
\text { overall } 80 / 405(20 \%), 45 / 224(20 \%) \text { in- } \\
\text { tervention and } 35 / 181(19 \%) \text { control. Rea- } \\
\text { sons for loss to follow-up not provided. } \\
\text { Used intention-to-treat analysis }\end{array}$ \\
\hline Selective reporting (reporting bias) & Low risk & $\begin{array}{l}\text { Protocol available and all prespecified out- } \\
\text { comes reported }\end{array}$ \\
\hline Other bias & Unclear risk & Insufficient information available to assess \\
\hline Implementation Integrity & Unclear risk & Insufficient information available to assess \\
\hline $\begin{array}{l}\text { Blinding of participants and personnel } \\
\text { (performance bias) } \\
\text { All outcomes }\end{array}$ & High risk & $\begin{array}{l}\text { Participants and personnel could not be } \\
\text { blinded, outcome likely to be influenced by } \\
\text { lack of blinding }\end{array}$ \\
\hline $\begin{array}{l}\text { Blinding of outcome assessment (detection } \\
\text { bias) } \\
\text { All outcomes }\end{array}$ & Low risk & $\begin{array}{l}\text { Assessor was not aware of treatment alloca- } \\
\text { tion }\end{array}$ \\
\hline
\end{tabular}


Methods

Participants
Study design: Pilot randomised controlled trial

Setting: Specialist

Diagnosis: Met study criteria for major depression or dysthymia or had persistent depressive symptoms at both baseline and 1 month later

Inclusion criteria: Low-income, Latina women at least 3 months past initial diagnosis (to avoid recruiting women with adjustment disorder) with carcinoma of the cervix or breast cancer (stage I-IV) receiving care in the outpatient breast and gynaecology clinics Exclusion criteria: In palliative care, suicidal, history of bipolar or psychotic disorder , evidence of gross cognitive impairment, currently abusing drugs or alcohol, currently receiving psychotherapy, or unable to speak Spanish or English

Age: Mean 47.25 years

Gender: 100\% female

Ethnicity: 100\% Latino

Country: United States

Sample size (randomised): Total participants 55, intervention 28, control 27
Interventions
Treatment: Collaborative care

Contains the four elements of collaborative care:

1) a multi-professional approach to patient care: Oncologist (PCP), social worker (CM) , psychiatrist (MH specialist)

2) a structured management plan: an initial assessment and education session linking the importance of depression treatment to cancer treatment adherence, overall health, and well-being. Patients were educated about ADs and manualised problem solving therapy (PST) and allowed to choose either. ADs included: a meeting of the patient, the PCP, and the $\mathrm{CM}$ to initiate ADs (according to modified treatment guidelines). PCPs provided medication follow-up for patients during regularly scheduled clinic visits, which the CM attended when possible. The CM used pre-printed forms to assess side effects, medication adherence, and depressive symptom severity; they then provided feedback to the PCP and the MH specialist. Patients who did not experience at least $50 \%$ reduction in depressive symptoms were scheduled for an in-person evaluation by the $\mathrm{MH}$ specialist to identify potential causes for persistent depressive symptoms and make treatment adjustments 3) scheduled patient follow-ups: PST = initial meeting then 8 weekly follow-ups, medication = regular scheduled visits with PCP which CM attended when possible and CM contacted patients at least every two weeks either face-to-face or by telephone

4) enhanced inter-professional communication: Joint CM and PCP meeting to initiate ADs, joint notes kept in medical record, same day telephone consultation available from MH specialist, CM provided feedback on AD follow-ups to PCP and MH specialist, biweekly supervision for $\mathrm{CM}$ by $\mathrm{MH}$ specialist, consultation with $\mathrm{MH}$ specialist for those not progressing fed back to PCP and CM

Control: Treatment as usual enhanced as patients were informed of their depression diagnosis and usual mental health resources available to them. The study recruiters suggested that they talk with their doctor or the clinic social worker and placed a note in the patient's medical record indicating the presence of depressive symptoms

Outcomes

Depression (Depression symptoms): 4, 8 months

Quality of Life (mental and physical health): 4, 8 months 


\section{Dwight-Johnson 2005 (Continued)}

\section{Risk of bias}

\begin{tabular}{|c|c|c|}
\hline Bias & Authors' judgement & Support for judgement \\
\hline $\begin{array}{l}\text { Random sequence generation (selection } \\
\text { bias) }\end{array}$ & Low risk & Computer generated \\
\hline Allocation concealment (selection bias) & Low risk & Sealed envelope \\
\hline $\begin{array}{l}\text { Incomplete outcome data (attrition bias) } \\
\text { All outcomes }\end{array}$ & Unclear risk & $\begin{array}{l}\text { Short-term loss to follow-up based on } \\
\text { primary depression outcome (depression } \\
\text { symptom improvement } \geq 50 \% \text { ) was: over- } \\
\text { all } 2 / 55(4 \%), 1 / 28(4 \%) \text { intervention and } \\
1 / 27(4 \%) \text { control. Reasons for loss to fol- } \\
\text { low-up not provided Used intention-to- } \\
\text { treat analysis }\end{array}$ \\
\hline Selective reporting (reporting bias) & Unclear risk & Insufficient information available to assess \\
\hline Other bias & Unclear risk & Insufficient information available to assess \\
\hline Implementation Integrity & Unclear risk & Insufficient information available to assess \\
\hline $\begin{array}{l}\text { Blinding of participants and personnel } \\
\text { (performance bias) } \\
\text { All outcomes }\end{array}$ & High risk & $\begin{array}{l}\text { Participants and personnel could not be } \\
\text { blinded, outcome likely to be influenced by } \\
\text { lack of blinding }\end{array}$ \\
\hline $\begin{array}{l}\text { Blinding of outcome assessment (detection } \\
\text { bias) } \\
\text { All outcomes }\end{array}$ & Low risk & $\begin{array}{l}\text { Assessor was not aware of treatment alloca- } \\
\text { tion }\end{array}$ \\
\hline
\end{tabular}

Dwight-Johnson 2010

\begin{tabular}{ll} 
Methods & Study design: Randomised controlled trial \\
\hline Participants & Setting: Primary care \\
& Diagnosis: Probable major depressive disorder or dysthymia, which was determined by \\
& using the Primary care evaluation of Mental Disorders (PRIME-MD) and Patient Health \\
& Questionnaire-9 (PHQ-9) \\
Inclusion criteria: Low income Latino patients aged 18 or older and able to speak English \\
or Spanish \\
Exclusion criteria: Probable bipolar disorder, cognitive impairment, lifetime psychotic \\
symptoms or disorder, or suicidal ideation \\
Age: Mean 49.8 (SD 12.6) years \\
Gender: $84 \%$ female \\
Ethnicity: $100 \%$ Latino \\
Country: United States
\end{tabular}


Dwight-Johnson 2010 (Continued)

Sample size (randomised): Total participants 339, intervention 173, control 166

\begin{tabular}{|c|c|c|}
\hline Interventions & \multicolumn{2}{|c|}{$\begin{array}{l}\text { Treatment: Collaborative care } \\
\text { Contains the four elements of collaborative care: } \\
\text { 1) a multi-professional approach to patient care: Primary care provider (PCP), social } \\
\text { worker (CM), psychiatrist (MH specialist) } \\
\text { 2) a structured management plan: CMs educated patients about depression and its treat- } \\
\text { ment, elicited treatment preferences, and provided active outreach and systematic as- } \\
\text { sessment. Depending on patients' treatment preferences, the CM provided short-term } \\
\text { manualised CBT, supported AD medication treatment, or both. Supported AD treat- } \\
\text { ment involved encouraging adherence, assessing side effects and treatment response, and } \\
\text { sharing this information with primary care providers. CBT was provided at no cost, and } \\
\text { prescriptions were filled at low or no cost } \\
\text { 3) scheduled patient follow-ups: CBT = once a week for } 12 \text { weeks, medication = about } \\
\text { every } 2 \text { weeks initially and then at least monthly after that } \\
\text { 4) enhanced inter-professional communication: CMs shared AD follow-up detail with } \\
\text { PCPs. MH specialist conducted weekly supervision by phone and with both CMs at } \\
\text { same time } \\
\text { Control: Treatment as usual enhanced as patients received a letter to take to PCP stating } \\
\text { that they had screened positive for depression, an educational pamphlet, and a list of } \\
\text { local mental health resources }\end{array}$} \\
\hline Outcomes & \multicolumn{2}{|c|}{$\begin{array}{l}\text { Depression (PHQ-9): } 4 \text { months } \\
\text { Medication use: } 4 \text { months }\end{array}$} \\
\hline Notes & \multicolumn{2}{|c|}{$\begin{array}{l}\text { AD: antidepressant; CBT: cognitive behaviour therapy; CM: case manager; } \mathrm{MH} \text { : mental } \\
\text { health; PCP: primary care provider; PHQ-9: Patient Health Questionnaire-9 }\end{array}$} \\
\hline \multicolumn{3}{|l|}{ Risk of bias } \\
\hline Bias & Authors' judgement & Support for judgement \\
\hline $\begin{array}{l}\text { Random sequence generation (selection } \\
\text { bias) }\end{array}$ & Unclear risk & Insufficient information available to assess \\
\hline Allocation concealment (selection bias) & Unclear risk & Insufficient information available to assess \\
\hline $\begin{array}{l}\text { Incomplete outcome data (attrition bias) } \\
\text { All outcomes }\end{array}$ & Unclear risk & $\begin{array}{l}\text { Short-term loss to follow-up based on pri- } \\
\text { mary depression outcome (PHQ-9) was } \\
\text { unclear. Reasons for loss to follow-up not } \\
\text { provided. Used intention-to-treat analysis }\end{array}$ \\
\hline Selective reporting (reporting bias) & Unclear risk & Insufficient information available to assess \\
\hline Other bias & Unclear risk & Insufficient information available to assess \\
\hline Implementation Integrity & Unclear risk & Insufficient information available to assess \\
\hline
\end{tabular}

Collaborative care for depression and anxiety problems (Review) 
Dwight-Johnson 2010 (Continued)

Blinding of participants and personnel High risk (performance bias)

All outcomes
Participants and personnel could not be blinded, outcome likely to be influenced by lack of blinding

Insufficient information available to assess

Blinding of outcome assessment (detection Unclear risk

bias)

All outcomes

\section{Dwight-Johnson 2011}

Methods

Participants
Study design: Pilot randomised controlled trial

Setting: Primary care

Diagnosis: Probable major depressive disorder assessed using the PHQ-9. Criteria were the reporting of a minimum of five of the nine symptoms assessed and a cut-off score of 10

Inclusion criteria: Rural areas, self-identified as Latino, spoke English or Spanish

Exclusion criteria: Bipolar disorder, cognitive impairment, current or lifetime psychotic symptoms or disorder, current substance abuse, acute suicidal ideation

Age: Mean 39.8 (SD 10.56) years

Gender: $78 \%$ female

Ethnicity: 91\% Mexican

Country: United States

Sample size (randomised): Total participants 101, intervention 50, control 51
Treatment: Telephone CBT

Contains the four elements of collaborative care:

1) a multi-professional approach to patient care: Primary care provider (PCP), social workers (CM), social workers, psychiatrist, and psychologist/psychiatrist (MH specialist) 2) a structured management plan: Initial structured assessment of clinical history, motivation for treatment, and use of strategies to enhance patients' motivation to engage in treatment (this session could be face-to-face). Each session focused on a chapter from a patient workbook that had been translated into the Spanish language and made culturally relevant. The sessions emphasised behavioural activation and strategies for identifying, interrupting, and distancing oneself from negative thoughts. Each session included structured assessment of depressive symptoms, review of the previous session, debriefing of homework assignment, introduction of new material, description of the new homework assignment, and a motivational assessment and enhancement exercise focused on the homework assignment. If indicated, CMs made brief supportive telephone contacts between sessions and could refer the patient for case management services for depression care needs, such as assistance in making appointments with clinic providers and referrals to community services. The CM did not take an active role in management of ADs but could discuss medication as a treatment option and ask about medication adherence all questions related to medication were referred back to PCP

3) scheduled patient follow-ups: $\mathrm{CBT}=8$ telephone sessions.

4) enhanced inter-professional communication: CMs liaised with PCPs when required in relation to medication. Suicide safety plans when necessary were communicated to PCP. CMs had weekly supervision with a team of MH specialists 
Dwight-Johnson 2011 (Continued)

Control: Treatment as usual enhanced as patients were encouraged to talk with their PCP about depression treatment and PCPs received a letter informing them of their patient's depression status and study enrolment

\begin{tabular}{ll} 
Outcomes & $\begin{array}{l}\text { Depression (HSCL and PHQ-9): } 6 \text { weeks, 3, } 6 \text { months } \\
\text { Satisfaction: } 6 \text { weeks, 3, } 6 \text { months }\end{array}$ \\
\hline Notes & $\begin{array}{l}\text { AD: antidepressant; CBT: cognitive behaviour therapy; CM: case manager; HSCL: Hop- } \\
\text { kins Sympton Checklist; MH: mental health; PCP: primary care provider; PHQ-9: Pa- } \\
\text { tient Health Questionnaire-9 }\end{array}$
\end{tabular}

\section{Risk of bias}

\begin{tabular}{|c|c|c|}
\hline Bias & Authors' judgement & Support for judgement \\
\hline $\begin{array}{l}\text { Random sequence generation (selection } \\
\text { bias) }\end{array}$ & Unclear risk & Stratified permuted-block randomisation \\
\hline Allocation concealment (selection bias) & Low risk & Allocation revealed by telephone \\
\hline $\begin{array}{l}\text { Incomplete outcome data (attrition bias) } \\
\text { All outcomes }\end{array}$ & Unclear risk & $\begin{array}{l}\text { Short-term loss to follow-up based on pri- } \\
\text { mary depression outcome (PHQ-9) was: } \\
\text { overall 24/101 (24\%), 8/50 (16\%) inter- } \\
\text { vention and } 16 / 51(31 \%) \text { control. Reasons } \\
\text { for loss to follow-up not provided. Inten- } \\
\text { tion-to-treat analysis reported based on the } \\
\text { assumption data is missing at random }\end{array}$ \\
\hline Selective reporting (reporting bias) & Unclear risk & Insufficient information available to assess \\
\hline Other bias & Unclear risk & Insufficient information available to assess \\
\hline Implementation Integrity & Low risk & $\begin{array}{l}\text { Attempts were made to assess implemen- } \\
\text { tation integrity (e.g. direct observation or } \\
\text { rating of tapes) }\end{array}$ \\
\hline $\begin{array}{l}\text { Blinding of participants and personnel } \\
\text { (performance bias) } \\
\text { All outcomes }\end{array}$ & High risk & $\begin{array}{l}\text { Participants and personnel could not be } \\
\text { blinded, outcome likely to be influenced by } \\
\text { lack of blinding }\end{array}$ \\
\hline $\begin{array}{l}\text { Blinding of outcome assessment (detection } \\
\text { bias) } \\
\text { All outcomes }\end{array}$ & Low risk & $\begin{array}{l}\text { Assessor was not aware of treatment alloca- } \\
\text { tion }\end{array}$ \\
\hline
\end{tabular}


Methods

Participants
Study design: Randomised controlled trial

Setting: Community

Diagnosis: Screened positive for clinically significant depression

Inclusion criteria: Home care, 65 and older

Exclusion criteria: significant cognitive impairment, participation in another depression study

Age: $=$ Mean 78.1 years

Gender: $73 \%$ female

Ethnicity: $72 \%$ white

Country: United States

Sample size (randomised): Total participants 311, intervention 155, control 156

Treatment: Stepped care decision support

Contains the four elements of collaborative care:

1) a multi-professional approach to patient care: Primary care physician (PCP), existing staff - nurses, social workers, psychiatric nurses, a telephone case manager and a psychologist (CM), existing staff - psychiatrist, nurses, social workers (MH specialist)

2) a structured management plan: A stepped care algorithm (based on IMPACT) in which patients were offered a choice of PST or ADs prescribed by PCP, or combined treatment if indicated. Step 1 (8-10 weeks) choice of AD or PST. Patients with full response go to maintenance treatment. Step 2 (4-8 weeks): if AD in step 1 and partial response give different $\mathrm{AD}$ type or augment $\mathrm{AD}$, if no response PST. If PST in step 1 and partial response add $\mathrm{AD}$ or different $\mathrm{AD}$ type, if no response give $\mathrm{AD}$. CMs monitored medication and delivered structured PST

3) scheduled patient follow-ups: PST = 6-12 sessions, medication = as per stepped care algorithm

4) enhanced inter-professional communication: CM communicated with PCP about medication or if a patient did not improve. Usual supervisory staff had the responsibility of monitoring and supportive supervision

Control: Treatment as usual was enhanced by routine depression screening and staff training in depression care management for older adults. Patients PCP informed if a patient screened positive for probable major or minor depression

Outcomes

Depression (PHQ-9): 4, 8, 12 months

Medication use: during study period

Quality of Life (mental and physical health): 4, 8, 12 months

Notes

AD: antidepressant; $\mathrm{CM}$ : case manager; $\mathrm{MH}$ : mental health; PCP: primary care provider; PHQ-9: Patient Health Questionnaire-9; PST: problem solving therapy

\section{Risk of bias}

\begin{tabular}{l|l|l} 
Bias & Authors' judgement & Support for judgement \\
\hline $\begin{array}{l}\text { Random sequence generation (selection } \\
\text { bias) }\end{array}$ & Unclear risk & Insufficient information available to assess \\
\hline Allocation concealment (selection bias) & Unclear risk & Insufficient information available to assess
\end{tabular}

Collaborative care for depression and anxiety problems (Review) 
Ell 2007 (Continued)

\begin{tabular}{|c|c|c|}
\hline $\begin{array}{l}\text { Incomplete outcome data (attrition bias) } \\
\text { All outcomes }\end{array}$ & Unclear risk & $\begin{array}{l}\text { Short-term loss to follow-up based on pri- } \\
\text { mary depression outcome (PHQ-950\% re- } \\
\text { duction) was: overall 113/311 (36\%), 58/ } \\
156(37 \%) \text { intervention and 55/155 (35\%) } \\
\text { control. Reasons for loss to follow-up not } \\
\text { provided across groups. Intention-to-treat } \\
\text { analysis reported using observed data }\end{array}$ \\
\hline Selective reporting (reporting bias) & Unclear risk & Insufficient information available to assess \\
\hline Other bias & Unclear risk & Insufficient information available to assess \\
\hline Implementation Integrity & Low risk & $\begin{array}{l}\text { Attempts were made to assess implemen- } \\
\text { tation integrity (e.g. direct observation or } \\
\text { rating of tapes) }\end{array}$ \\
\hline $\begin{array}{l}\text { Blinding of participants and personnel } \\
\text { (performance bias) } \\
\text { All outcomes }\end{array}$ & High risk & $\begin{array}{l}\text { Participants and personnel could not be } \\
\text { blinded, outcome likely to be influenced by } \\
\text { lack of blinding }\end{array}$ \\
\hline $\begin{array}{l}\text { Blinding of outcome assessment (detection } \\
\text { bias) } \\
\text { All outcomes }\end{array}$ & Unclear risk & Insufficient information available to assess \\
\hline
\end{tabular}

\section{Ell 2008}

Methods

Participants
Study design: Randomised controlled trial

Setting: Specialist

Diagnosis: One of the two cardinal depression symptoms more than half of the days to nearly every day plus a PHQ-9 depression scale score of greater than or equal to 10 indicating major depression and/or two questions from the Structured Clinical Interview for Diagnostic and Statistical Manual of Mental Disorders, 4th Edition indicating dysthymia

Inclusion criteria: Low income, greater than or equal to 90 days after cancer diagnosis and receiving acute or follow-up care in oncology clinics, 18 years or older

Exclusion criteria: Acute suicidal ideation, advanced cancer or other condition that limited remaining life expectancy to less than 6 months, a score of 8 or greater on the Alcohol Use Disorders Identification Test alcohol assessment, recently used lithium/antipsychotic medication, a self-reported adaptation of the Karnofsky Performance Status Scale score of 2 or less on an 11-point scale representing severe functional impairment in cancer patients and inability to speak English or Spanish

Age: $49.4 \% \geq 50$ years

Gender: $84 \%$ female

Ethnicity: $88 \%$ Hispanic

Country: United States

Sample size (randomised): Total participants 472, intervention 242, control 230 


Treatment: Alleviating Depression Among Patients with Cancer (ADAPt-C)
Contains the four elements of collaborative care:
1) a multi-professional approach to patient care: Oncologist (PCP), social workers (CM)
, psychiatrist (MH specialist)
2) a structured management plan: A stepped care algorithm (based on IMPACT) in
which patients were randomised to AD or PST or combined. The algorithm included
CMs who provided psychotherapy and community services navigation (with assistance
from patient navigators) through a personalised treatment plan that included patient AD
or PST preferences, stepped care management and protocol for PST and CM telephone
maintenance/relapse prevention
3) scheduled patient follow-ups: PST= 6-12 weekly sessions, ADs = had as required
appointments with psychiatrist. In maintenance CM telephoned patients monthly for
up to 12 months post-treatment initiation
4) enhanced inter-professional communication: The CM communicates with the PCP
as needed and interacts via written notes or verbally. PCP provides maintenance pre-
scriptions in consultation with MH specialist. MH specialist provides weekly telephone
supervision to review the CMs caseload
Control: Treatment as usual enhanced by patient/family depression and cancer educa-
tional pamphlets and a listing of centre/community financial, social services, transporta-
tion, and childcare resources. The treating PCP was informed of patients depression
status

Outcomes

Depression (PHQ-9): 6, 12, 18, 24 months

Medication use: 12, 18, 24 months

Quality of Life (mental and physical health): 6, 12, 18, 24 months

Satisfaction: 18, 24 months

Notes

AD: antidepressant; CM: case manager; $\mathrm{MH}$ : mental health; PCP: primary care provider;

PHQ-9: Patient Health Questionnaire-9; PST: problem solving therapy

Risk of bias

\begin{tabular}{|c|c|c|}
\hline Bias & Authors' judgement & Support for judgement \\
\hline $\begin{array}{l}\text { Random sequence generation (selection } \\
\text { bias) }\end{array}$ & Low risk & Computer generated \\
\hline Allocation concealment (selection bias) & Low risk & Patients chose one of five sealed envelopes \\
\hline $\begin{array}{l}\text { Incomplete outcome data (attrition bias) } \\
\text { All outcomes }\end{array}$ & Unclear risk & $\begin{array}{l}\text { Short-term loss to follow-up based on pri- } \\
\text { mary depression outcome (PHQ-9, 50\% } \\
\text { reduction) was: overall } 154 / 472(33 \%) \text {, } \\
76 / 242(31 \%) \text { intervention and } 78 / 230 \\
(34 \%) \text { control. Reasons for loss to follow- } \\
\text { up provided, similar reasons for missing } \\
\text { data across groups. Intention-to-treat anal- } \\
\text { ysis reported using available data and they } \\
\text { also conducted analyses using multiple im- }\end{array}$ \\
\hline
\end{tabular}


Ell 2008 (Continued)

putation methods

\begin{tabular}{l|ll}
\hline Selective reporting (reporting bias) & Low risk & $\begin{array}{l}\text { Protocol available and all prespecified out- } \\
\text { comes reported }\end{array}$ \\
\hline Other bias & Unclear risk & Insufficient information available to assess \\
\hline Implementation Integrity & Low risk & $\begin{array}{l}\text { Attempts were made to assess implemen- } \\
\text { tation integrity (e.g. direct observation or } \\
\text { rating of tapes) }\end{array}$ \\
\hline
\end{tabular}

Blinding of participants and personnel High risk (performance bias)

Participants and personnel could not be All outcomes blinded, outcome likely to be influenced by lack of blinding

Blinding of outcome assessment (detection Low risk bias)

Assessor was not aware of treatment alloca-

All outcomes

\section{Ell 2010}

Methods

Participants
Study design: Randomised controlled trial

Setting: Community and primary care

Diagnosis: One of two cardinal depression symptoms more than half the days to nearly every day and scored greater than or equal to 10 on the PHQ-9, indicating a high likelihood of clinically significant depression

Inclusion criteria: Low income and predominantly Hispanic, patients with diabetes, aged greater than or equal to 18 years

Exclusion criteria: Acute suicidal ideation, a score of greater than or equal to 8 on the $\mathrm{Al}$ cohol Use Disorders Identification Test alcohol assessment, recent lithium/antipsychotic medication use, and inability to speak English or Spanish

Age: $72.1 \% \geq 50$ years

Gender: $82 \%$ female

Ethnicity: 96\% Hispanic

Country: United States

Sample size (randomised): Total participants 387, intervention 193, control 194
Interventions
Treatment: Multifaceted Diabetes and Depression Programme (MDDP)

Contains the four elements of collaborative care:

1) a multi-professional approach to patient care: Primary care physician (PCP), social workers (CM), psychiatrist ( $\mathrm{MH}$ specialist)

2) a structured management plan: CM conducted assessment and implemented steppedcare algorithm including 1) culturally adapted PST designed to enhance diabetes and depression self-management and coping with socioeconomic stress provided by CM and/ or ADs prescribed by PCP; 2) CM monthly telephone follow-up symptom monitoring, treatment maintenance, and relapse prevention; and 3) care and service system navigation by the $\mathrm{CM}$ and an assistant patient navigator. The algorithm included the following: 
Step 1 (weeks 1-8): based on patient preference PST or AD, Step 2 (weeks 9 -12): patients with partial/non-response receive a different $\mathrm{AD}$ or the addition of $\mathrm{AD}$ or PST, Step 3: patients with full response move to monthly maintenance/relapse prevention telephone monitoring involving monitoring depressive symptoms, provide BA support for engaging in pleasant activities and motivational support for ongoing use of PST skills and medication adherence, and invites to attend an open-ended PST support group. Nonresponsive patients were considered for additional PST, augmentation of low-dose Trazodone for insomnia, and referral to specialty mental health care

3) scheduled patient follow-ups: Acute treatment: bimonthly PST and AD monitoring over 4 months $=8-12$ sessions plus booster sessions if indicated. Follow-up monthly telephone calls by CM for up to 12 months. A PST open-ended patient support group available up to 12 months post-treatment

4) enhanced inter-professional communication: $\mathrm{MH}$ specialist was available to $\mathrm{CM}$ and PCP via pager and provided weekly telephone CM supervision. $\mathrm{MH}$ specialist recommendations were communicated by the $\mathrm{CM}$ to the PCP and, if requested, the $\mathrm{MH}$ specialist provided PCP medication telephone consultation

Control: Treatment as usual enhanced as patients were given patient and family-focused depression educational pamphlets and a community, financial, social services, transportation, and child care resource list. PCPs were informed of patient depression diagnoses

Outcomes

Depression (HSCL and PHQ-9): 6, 12, 18, 24 months

Medication use: 6, 12, 18, 18-24 months

Quality of Life (mental and physical health): 6, 12, 18, 24 months

Satisfaction: 24 months

Notes

CM: case manager; HSCL: Hopkins Sympton Checklist; MH: mental health; PCP: primary care provider; PHQ-9: Patient Health Questionnaire-9; PST: problem solving therapy

\section{Risk of bias}

\begin{tabular}{l|l|l}
\hline Bias & Authors judgement & Support for judgement \\
\hline $\begin{array}{l}\text { Random sequence generation (selection } \\
\text { bias) }\end{array}$ & Low risk & Computer generated in blocks of 10 \\
\hline Allocation concealment (selection bias) & Low risk & $\begin{array}{l}\text { Standard block size. Patients chose one of } \\
\text { five sealed envelopes }\end{array}$ \\
\hline
\end{tabular}

Incomplete outcome data (attrition bias) Unclear risk All outcomes
Short-term loss to follow-up based on primary depression outcome (HSCL-20 50\% reduction) was: overall $85 / 387(22 \%), 42 /$ $193(22 \%)$ intervention and 43/194 (22\%) control. Reasons for loss to follow-up provided, similar reasons for missing data across groups. Intention-to-treat analysis reported using available data and also conducted analyses using multiple imputation methods 


\begin{tabular}{l|l|l}
\hline Selective reporting (reporting bias) & Low risk & $\begin{array}{l}\text { Protocol available and all prespecified out- } \\
\text { comes reported }\end{array}$ \\
\hline Other bias & Unclear risk & Insufficient information available to assess \\
\hline $\begin{array}{l}\text { Implementation Integrity } \\
\begin{array}{l}\text { Blinding of participants and personnel } \\
\text { (performance bias) } \\
\text { All outcomes }\end{array}\end{array}$ & High risk & Insufficient information available to assess \\
\hline $\begin{array}{l}\text { Blinding of outcome assessment (detection } \\
\text { bias) } \\
\text { All outcomes }\end{array}$ & Low risk & $\begin{array}{l}\text { Participants and personnel could not be } \\
\text { blinded, outcome likely to be influenced by } \\
\text { lack of blinding }\end{array}$ \\
\hline
\end{tabular}

\section{Finley 2003}

Methods

Participants
Study design: Randomised controlled trial

Setting: Primary care

Diagnosis: Depressive symptoms and just commenced antidepressant therapy to treat this

Inclusion criteria: Not stated

Exclusion criteria: Evidence that subjects had received an antidepressant during the preceding 6 months; concurrent psychiatric or psychological treatment; current symptoms of mania or bipolar disorder; psychotic symptoms; eminent suicidality; and active substance abuse or dependence. If psychiatric treatment was indicated at baseline or any time during the investigation, subjects were referred to the HMO's psychiatry department for care (or were permitted to self-refer) and subsequently were excluded from further study participation

Age: Mean 54.3 years

Gender: 85\% female

Ethnicity: Not stated

Country: United States

Sample size (randomised): Total participants 125, intervention 75, control 50
Intervention: Collaborative care

Contains the four elements of collaborative care:

1) a multi-professional approach to patient care: Primary care provider (PCP), pharmacist (CM), psychiatrist ( $\mathrm{MH}$ specialist)

2) a structured management plan: Assessment of severity of psychopathology, potential stressors and other predisposing factors and patient education was provided. Information on depression and the role of ADs was presented (including potential therapeutic effects/ adverse effects). Patients were advised of other treatment options and resources available. $\mathrm{CMs}$ were permitted to titrate $\mathrm{ADs}$ consistent with clinical practice guidelines. $\mathrm{CMs}$ could also prescribe ancillary drugs but if a change in $\mathrm{AD}$ drugs was indicated, approval from the PCP was required. As patients improved CMs identified neglected activities and encouraged patients to resume them. Patients were advised to contact the clinic if 
Finley 2003 (Continued)

they were considering the discontinuation of antidepressants at any time in the future 3) scheduled patient follow-ups: Assessment plus 5 telephone calls at key junctures in recovery process and 2 clinic visits at 6 and 24 weeks

4) enhanced inter-professional communication: All contacts were recorded in the medical record in the form of a detailed progress note. CM discussed with PCP by phone or messaging system any need for change to ADs. At the end of treatment a comprehensive summary of the treatment course and patient disposition was entered into records. Weekly case conferences with CMs and MH specialist clarified diagnostic issues and more clearly delineate treatment plans. $\mathrm{MH}$ specialists were also available for off-site telephone consultation on an as-needed basis for more pertinent issues

Control: Treatment as usual including brief information on the $\mathrm{AD}$, therapeutic end points, and side effects in a manner consistent with patient education routinely delivered by the pharmacy. The referring PCP was notified of assignment and subsequent treatment and follow-up were left to the provider's discretion

Outcomes

Depression (BIDS): 6 months

Medication use: 114 days, 231 days, 3, 6, months

Satisfaction: 6 months

Notes

AD: antidepressant; CM: case manager; $\mathrm{MH}$ : mental health; PCP: primary care provider

\section{Risk of bias}

\begin{tabular}{|c|c|c|}
\hline Bias & Authors' judgement & Support for judgement \\
\hline $\begin{array}{l}\text { Random sequence generation (selection } \\
\text { bias) }\end{array}$ & Unclear risk & Insufficient information available to assess \\
\hline Allocation concealment (selection bias) & Low risk & Sealed envelope \\
\hline $\begin{array}{l}\text { Incomplete outcome data (attrition bias) } \\
\text { All outcomes }\end{array}$ & Unclear risk & $\begin{array}{l}\text { Short-term loss to follow-up based on pri- } \\
\text { mary depression outcome (Percentage with } \\
50 \% \text { reduction) was: overall } 47 / 125 \text { (38\%) } \\
, 21 / 75 \text { (28\%) intervention and } 26 / 50 \\
(52 \%) \text { control. Reasons for loss to follow- } \\
\text { up not reported Intention-to-treat analy- } \\
\text { sis reported, no description of methods to } \\
\text { manage missing data }\end{array}$ \\
\hline Selective reporting (reporting bias) & Unclear risk & Insufficient information available to assess \\
\hline Other bias & Unclear risk & Insufficient information available to assess \\
\hline Implementation Integrity & Unclear risk & Insufficient information available to assess \\
\hline $\begin{array}{l}\text { Blinding of participants and personnel } \\
\text { (performance bias) } \\
\text { All outcomes }\end{array}$ & High risk & $\begin{array}{l}\text { Participants and personnel could not be } \\
\text { blinded, outcome likely to be influenced by } \\
\text { lack of blinding }\end{array}$ \\
\hline
\end{tabular}


Finley 2003 (Continued)

Blinding of outcome assessment (detection Unclear risk

Insufficient information available to assess bias)

All outcomes

Fortney 2007

Methods

Study design: Cluster-randomised controlled trial

Participants

Setting: Primary care

Diagnosis: Screened positive for depression, defined as a PHQ-9 score $\geq 12$

Inclusion criteria: Veterans

Exclusion criteria: Diagnosis of schizophrenia, current suicidal ideation, recent bereavement, pregnancy, a court-appointed guardian, substance dependence, bipolar disorder, cognitive impairment, or receiving specialty mental health treatment

Age: Mean 59.2 (SD 12.2) years

Gender: 8\% female

Ethnicity: $75 \%$ white

Country: United States

Sample size (randomised): Total clusters 7, intervention 3, control 4; Total participants 395 , intervention 177 , control 218

Interventions

Intervention: Stepped care Telemedicine Enhanced Antidepressant Management (TEAM)

Contains the four elements of collaborative care:

1) a multi-professional approach to patient care: Primary care provider (PCP), nurse supported by pharmacist (CM), psychiatrist (MH specialist)

2) a structured management plan: stepped-care model including: Step (1) choice of either watchful waiting or $\mathrm{AD}$. CM encounters were conducted via telephone and were scripted and administered using software package. During the initial care management encounter, patients were: (1) clinically assessed; (2) educated and activated; and 3) assessed for treatment barriers. Follow-up encounters monitored symptoms, medication adherence, and side-effects. Step (2) If the patient did not respond to the initial AD, the pharmacist conducted a medication history and provided pharmacotherapy recommendations to PCPs via an electronic progress note. The pharmacist also provided nonscripted medication management over the phone to patients experiencing severe sideeffects or problems with non-adherence. Step (3) If the patient did not respond to 2 $\mathrm{AD}$ trials, the protocol was to recommend a telepsychiatry consultation followed by additional treatment recommendations to the PCP

3) scheduled patient follow-ups: Acute = Telephone calls scheduled every 2 weeks Watchful waiting or continuation $=$ every 4 weeks

4) enhanced inter-professional communication: All feedback was provided to PCPs using the electronic medical record. Progress notes reporting failed trials requested an electronic co-signature from the PCP. CM had weekly supervision with $\mathrm{MH}$ specialist and pharmacist. Telepsychiatry consultation was followed by additional treatment recommendations to the PCP

Control: Treatment as usual enhanced as provider education (via interactive video and website) and patient education (via mail and website) were provided. Depression screening results were entered into the electronic medical record 
Fortney 2007 (Continued)

\begin{tabular}{|c|c|c|}
\hline Outcomes & \multicolumn{2}{|c|}{$\begin{array}{l}\text { Depression (HSCL-20): 6, } 12 \text { months } \\
\text { Medication use: } 6,12 \text { months } \\
\text { Quality of Life (mental and physical health): 6, } 12 \text { months } \\
\text { Satisfaction: } 6,12 \text { months }\end{array}$} \\
\hline Notes & \multicolumn{2}{|c|}{$\begin{array}{l}\text { CM: case manager; HSCL: Hopkins Sympton Checklist; MH: mental health; PCP: } \\
\text { primary care provider: PHQ-9: Patient Health Questionnaire; SD: standard deviation }\end{array}$} \\
\hline \multicolumn{3}{|l|}{ Risk of bias } \\
\hline Bias & Authors' judgement & Support for judgement \\
\hline $\begin{array}{l}\text { Random sequence generation (selection } \\
\text { bias) }\end{array}$ & Unclear risk & Insufficient information available to assess \\
\hline Allocation concealment (selection bias) & Unclear risk & Insufficient information available to assess \\
\hline $\begin{array}{l}\text { Incomplete outcome data (attrition bias) } \\
\text { All outcomes }\end{array}$ & Low risk & $\begin{array}{l}\text { Short-term loss to follow-up based on } \\
\text { primary depression outcome (Response } \\
\text { HSCL-20 50\% improvement) was: overall } \\
35 / 395(9 \%), 17 / 177(10 \%) \text { intervention } \\
\text { and } 18 / 218(8 \%) \text { control. Reasons for loss } \\
\text { to follow-up provided, with similar reasons } \\
\text { across groups. Used intention-to-treat anal- } \\
\text { ysis }\end{array}$ \\
\hline Selective reporting (reporting bias) & Low risk & $\begin{array}{l}\text { Protocol available and all prespecified out- } \\
\text { comes reported }\end{array}$ \\
\hline Other bias & Unclear risk & Insufficient information available to assess \\
\hline Implementation Integrity & Unclear risk & Insufficient information available to assess \\
\hline $\begin{array}{l}\text { Blinding of participants and personnel } \\
\text { (performance bias) } \\
\text { All outcomes }\end{array}$ & High risk & $\begin{array}{l}\text { Participants and personnel could not be } \\
\text { blinded, outcome likely to be influenced by } \\
\text { lack of blinding }\end{array}$ \\
\hline $\begin{array}{l}\text { Blinding of outcome assessment (detection } \\
\text { bias) } \\
\text { All outcomes }\end{array}$ & Low risk & $\begin{array}{l}\text { Assessor was not aware of treatment alloca- } \\
\text { tion }\end{array}$ \\
\hline
\end{tabular}


Methods

Participants
Study design: Randomised controlled trial

Setting: Primary care

Diagnosis: Major depression

Inclusion criteria: Adult women age 18-70 with major depression, without treatment in last 3 months and at least one child between 6 and 16 living with her

Exclusion criteria: Alcohol or drug abuse, previous bipolar, current or past psychotic symptoms, mental or physical disability which impedes their ability to participate in activities and evaluations of the study, pregnancy and high risk of suicide

Age: Mean 37.4 years

Gender: $100 \%$ female

Ethnicity: Not stated

Country: Chile

Sample size (randomised): Total Participants 345, intervention 175, control 170
Interventions
Intervention: Pharmacological intervention

Contains the four elements of collaborative care:

1) a multi-professional approach to patient care: Primary care provider (PCP), nonprofessional (CM), psychiatrist (MH specialist)

2) a structured management plan: A medication adherence programme via telephone where the $\mathrm{CM}$ used a protocol to provide education about medication, monitor adherence and side effects and reinforce attendance to PCP

3) scheduled patient follow-ups: 6 telephone calls at 2, 4, 6, 8, 10, 12 weeks

4) enhanced inter-professional communication: Psychiatrist and PCP had monthly meetings to discuss cases

Control: Treatment as usual enhanced by consultation with PCP, pharmacotherapy, individual or group psychotherapy with psychologists at clinics, referral to psychiatrist

Outcomes

Depression (HRSD): 3, 6 months

Medication use: 3, 6 months

Quality of Life (mental and physical health): 3, 6 months

Notes

CM: case manager; HRSD: Hamilton Rating Scale for Depression; MH: mental health; PCP: primary care provider

\section{Risk of bias}

\begin{tabular}{|c|c|c|}
\hline Bias & Authors' judgement & Support for judgement \\
\hline $\begin{array}{l}\text { Random sequence generation (selection } \\
\text { bias) }\end{array}$ & Low risk & Computer generated \\
\hline Allocation concealment (selection bias) & Low risk & Centrally allocated \\
\hline $\begin{array}{l}\text { Incomplete outcome data (attrition bias) } \\
\text { All outcomes }\end{array}$ & Unclear risk & $\begin{array}{l}\text { Short-term loss to follow-up based on pri- } \\
\text { mary depression outcome (HRSD) was: } \\
\text { overall } 71 / 345(21 \%), 32 / 175(18 \%) \text { in- } \\
\text { tervention and } 39 / 170(23 \%) \text { control. Rea- } \\
\text { sons for loss to follow-up not reported. In- }\end{array}$ \\
\hline
\end{tabular}


Fritsch 2007 (Continued)

tention-to-treat analysis reported, no description of methods to manage missing data

\begin{tabular}{lll}
\hline Selective reporting (reporting bias) & Unclear risk & Insufficient information available to assess \\
\hline Other bias & Unclear risk & Insufficient information available to assess \\
\hline $\begin{array}{l}\text { Implementation Integrity } \\
\begin{array}{l}\text { Blinding of participants and personnel } \\
\text { (performance bias) } \\
\text { All outcomes }\end{array}\end{array}$ & High risk & Insufficient information available to assess \\
\hline
\end{tabular}

Blinding of outcome assessment (detection Unclear risk bias)

All outcomes

Gensichen 2009

Methods

Participants
Study design: Cluster-randomised controlled trial

Setting: Primary care

Diagnosis: Diagnosis of major depression with indication for any antidepressive treatment. Diagnosis of major depression was based on a score of more than 9 points and a categorical diagnosis in the PHQ-9, and was confirmed by the family physician by using the checklists in the Diagnostic and Statistical Manual of Mental Disorders (DSM-IV), and International Classification of Diseases (ICD-10)

Inclusion criteria: Age 18 to 80 years, access to a private telephone, ability to give informed consent, and ability to communicate in German

Exclusion criteria: Confirmed pregnancy, severe alcohol or illicit drug consumption, or acute suicidal ideation assessed by the family physician

Age: Mean 51.1 years

Gender: $76 \%$ female

Ethnicity: Not stated

Country: Germany

Sample size (randomised): Total clusters 74, intervention 35, control 39; Total participants 626, intervention 310, control 316

Interventions
Intervention: Case management

Contains the four elements of collaborative care:

1) a multi-professional approach to patient care: Family physician (PCP), healthcare assistant (CM)

2) a structured management plan: CMs monitored depression symptoms and adherence to medication using a protocol. Having been trained in behavioural activation CMs encouraged patients to follow self-management activities, such as medication adherence and activation for pleasant or social activities

3) scheduled patient follow-ups: 19 telephone contacts twice weekly for first month then monthly for 11 months 
4) enhanced inter-professional communication: CMs provided PCP with information on patient's in a structured report

Control: Treatment as usual enhanced as PCPs received training on evidence-based depression treatment guidelines

\begin{tabular}{ll} 
Outcomes & $\begin{array}{l}\text { Depression (PHQ-9): 6,12 months } \\
\text { Medication use: } 12 \text { months } \\
\text { Quality of Life (mental and physical health): } 12 \text { months } \\
\text { Satisfaction: } 12 \text { months }\end{array}$ \\
\hline Notes & $\begin{array}{l}\text { CM: case manager; } M H: \text { mental health; PCP: primary care provider; PHQ-9:Patient } \\
\text { Health Questionnaire-9 }\end{array}$
\end{tabular}

\section{Risk of bias}

\begin{tabular}{|c|c|c|}
\hline Bias & Authors' judgement & Support for judgement \\
\hline $\begin{array}{l}\text { Random sequence generation (selection } \\
\text { bias) }\end{array}$ & Low risk & Computer generated \\
\hline Allocation concealment (selection bias) & High risk & $\begin{array}{l}\text { Central randomisation of clinic. Those re- } \\
\text { cruiting patients were aware of allocation }\end{array}$ \\
\hline $\begin{array}{l}\text { Incomplete outcome data (attrition bias) } \\
\text { All outcomes }\end{array}$ & Low risk & $\begin{array}{l}\text { Short-term loss to follow-up based on pri- } \\
\text { mary depression outcome (PHQ-9) was: } \\
\text { overall } 71 / 626(11 \%), 43 / 310(14 \%) \text { inter- } \\
\text { vention and } 28 / 316(9 \%) \text { control. Reasons } \\
\text { for loss to follow-up provided, with similar } \\
\text { reasons across groups. Used intention-to- } \\
\text { treat analysis }\end{array}$ \\
\hline Selective reporting (reporting bias) & Low risk & $\begin{array}{l}\text { Protocol available and all prespecified out- } \\
\text { comes reported }\end{array}$ \\
\hline Other bias & Unclear risk & Insufficient information available to assess \\
\hline Implementation Integrity & Unclear risk & Insufficient information available to assess \\
\hline $\begin{array}{l}\text { Blinding of participants and personnel } \\
\text { (performance bias) } \\
\text { All outcomes }\end{array}$ & High risk & $\begin{array}{l}\text { Participants and personnel could not be } \\
\text { blinded, outcome likely to be influenced by } \\
\text { lack of blinding }\end{array}$ \\
\hline $\begin{array}{l}\text { Blinding of outcome assessment (detection } \\
\text { bias) } \\
\text { All outcomes }\end{array}$ & High risk & $\begin{array}{l}\text { Assessor was potentially aware of treatment } \\
\text { allocation }\end{array}$ \\
\hline
\end{tabular}


Methods

Participants
Study design: Pilot randomised controlled trial

Setting: Primary care

Diagnosis: Women who became positive on Structured Clinical Interview for the Diagnostic and Statistical Manual of Mental Disorders (SCID) at 0 to 6 months postpartum. Participants were also given the opportunity to self-diagnose depression through a 9month survey question with a yes/no answer: "Since your baby was born, have you been depressed or diagnosed with depression?"

Inclusion criteria: Being a mother of a 0 - to 1 -month-old infant who was registered at one of the participating clinics, being English literate, and being greater than or equal to 12 years old

Exclusion criteria: Not stated

Age: Mean 27.6 years

Gender: $100 \%$ female

Ethnicity: $62 \%$ non-white

Country: United States

Sample size (randomised): Total participants 39, intervention 19, control 20

Intervention: Stepped collaborative care

Contains the four elements of collaborative care:

1) a multi-professional approach to patient care: Primary physician (PCP), nurse with $\mathrm{MH}$ experience (CM), psychiatrist ( $\mathrm{MH}$ specialist)

2) a structured management plan: The stepped care intervention included (1) referral to the PCP for initial treatment (AD and/or psychotherapy referral); (2) regular telephone follow-up with a CM; (3) decision support for PCPs (e.g. advice regarding specific ADs, additional treatment, or mental health referral); (4) consultation or referral to $\mathrm{MH}$ specialist for complex cases (e.g., psychiatrists conducted psychiatric evaluations and adjusted medications and therapists provided psychotherapy using CBT, interpersonal psychotherapy (IPT), or other therapies depending on patient need); and (5) patient education provided through the PCP, CM, and a mailed postpartum depression brochure. Telephone calls addressed depressive symptoms, mental health visits, treatment adherence and side effects, social support, suicidal ideation/plans, and lifestyle issues

3) scheduled patient follow-ups: 18 calls, conducted every two weeks until symptom remission for up to 9 months

4) enhanced inter-professional communication: The content of each call was documented on a form and a copy was faxed to the PCP. If a participant's symptoms were not resolving as expected, this was specifically communicated

Control: Treatment as usual enhanced as patients were informed of their depression diagnosis and referred to their PCP. The PCPs of some control patients were also PCPs of patients in the intervention arm

Outcomes

Depression (PHQ-9): 1, 9 months

Medication use: 9 months

Quality of Life (mental and physical health): 1, 2, 9 months

Satisfaction: 9 months

AD: antidepressant; CBT: cognitive behaviour therapy; CM: case manager; $\mathrm{MH}$ : mental health; PCP: primary care provider; PHQ-9: Patient Health Questionnaire-9 
Gjerdingen 2009 (Continued)

\section{Risk of bias}

\begin{tabular}{|c|c|c|}
\hline Bias & Authors' judgement & Support for judgement \\
\hline $\begin{array}{l}\text { Random sequence generation (selection } \\
\text { bias) }\end{array}$ & Low risk & Computer generated in blocks of 10 \\
\hline Allocation concealment (selection bias) & Unclear risk & $\begin{array}{l}\text { Standard block size. Insufficient informa- } \\
\text { tion available to assess }\end{array}$ \\
\hline $\begin{array}{l}\text { Incomplete outcome data (attrition bias) } \\
\text { All outcomes }\end{array}$ & Unclear risk & $\begin{array}{l}\text { Short-term loss to follow-up based on pri- } \\
\text { mary depression outcome (PHQ-9) was: } \\
\text { overall } 5 / 39(13 \%), 3 / 19(16 \%) \text { interven- } \\
\text { tion and } 2 / 20(10 \%) \text { control. Reasons for } \\
\text { loss to follow-up not provided. Intention } \\
\text { to treat not reported }\end{array}$ \\
\hline Selective reporting (reporting bias) & Unclear risk & Insufficient information available to assess \\
\hline Other bias & Unclear risk & Insufficient information available to assess \\
\hline Implementation Integrity & Unclear risk & Insufficient information available to assess \\
\hline $\begin{array}{l}\text { Blinding of participants and personnel } \\
\text { (performance bias) } \\
\text { All outcomes }\end{array}$ & High risk & $\begin{array}{l}\text { Participants and personnel could not be } \\
\text { blinded, outcome likely to be influenced by } \\
\text { lack of blinding }\end{array}$ \\
\hline $\begin{array}{l}\text { Blinding of outcome assessment (detection } \\
\text { bias) } \\
\text { All outcomes }\end{array}$ & Unclear risk & Insufficient information available to assess \\
\hline
\end{tabular}

Hedrick 2003

Methods

Participants
Study design: Cluster-randomised controlled trial

Setting: Primary care

Diagnosis: Current major depressive episode, dysthymia, or both. Assessed using Primary care evaluation of Mental Disorders (PRIME-MD) and additional questions from the Structured Clinical Interview for the Diagnostic and Statistical Manual of Mental Disorders (SCID)

Inclusion criteria: Veterans

Exclusion criteria: Recent visit to mental health specialty clinic or who had scheduled a future appointment, requiring treatment for substance abuse or PTSD prior to initiating depression treatment, acute suicidality, psychosis or other condition requiring immediate treatment

Age: Mean 57.2 years

Gender: 5\% female

Ethnicity: $80 \%$ caucasian 
Hedrick 2003 (Continued)

\section{Country: United States}

Sample size (randomised): Total clusters 4, intervention 2, control 2; Total participants 354, intervention 168 , control 186

Interventions

Intervention: Collaborative care

Contains the four elements of collaborative care:

1) a multi-professional approach to patient care: Primary care provider (PCP), social worker (CM), psychiatrist (MH specialist)

2) a structured management plan: The team (CM, MH specialist, psychologist and psychologist technician) met weekly to develop treatment plans and conduct 6 and 12 week progress evaluations for each patient. Using Veteran Affairs Major Depression Guidelines treatment options were: AD medication; add an adjunctive medication; a CBT group; schedule with the psychologist or psychiatrist; or refer to $\mathrm{MH}$ speciality care. Options were selected beginning with the least resource- intensive option based on previous treatments and patient preference. Patients were stepped up if non-response at 6 or 12 weeks. A videotape and workbook were mailed to each patient. CMs telephoned patients on a regular schedule to encourage adherence, address treatment barriers, and assess response

3) scheduled patient follow-ups: Acute $=3$ to 5 telephone calls, maintenance $=3$ to 5 calls, plus for those also receiving CBT $=6$ sessions

4) enhanced inter-professional communication: The team liaised with PCP re medication prescribing and treatment plans using electronic progress notes and if not acted upon the team contacted the PCP directly. The MH specialist contacted PCPs where there was question about treatment recommendations

Control: Consultation-liaison (CL) in which the PCP was responsible for initiating and coordinating the patient's care, with consultation from or referral to a psychiatrist if necessary. PCPs were notified of the patient's diagnosis and were able to refer patients to the psychiatrist, psychologist, and/or social workers, based in the organisation. The CL mental health providers provided treatment directly during individual visits with patients who were deemed manageable in the primary care setting

$\begin{array}{ll}\text { Outcomes } & \text { Depression (HSCL): 3, } 9 \text { months } \\ \text { Medication use: 3, 6, 9, } 12 \text { months } \\ \text { Quality of Life (mental and physical health): 3, } 9 \text { months } \\ \text { Satisfaction: 3, } 9 \text { months }\end{array}$

Notes

AD: antidepressant; CBT: cognitive behaviour therapy; CM: case manager; HSCL: Hopkins Sympton Checklist; MH: mental health; PCP: primary care provider

\section{Risk of bias}

\begin{tabular}{l|ll} 
Bias & Authors' judgement & Support for judgement \\
\hline $\begin{array}{l}\text { Random sequence generation (selection } \\
\text { bias) }\end{array}$ & Unclear risk & Insufficient information available to assess \\
\hline Allocation concealment (selection bias) & Unclear risk & Insufficient information available to assess
\end{tabular}


Hedrick 2003 (Continued)

\begin{tabular}{|c|c|c|}
\hline $\begin{array}{l}\text { Incomplete outcome data (attrition bias) } \\
\text { All outcomes }\end{array}$ & Unclear risk & $\begin{array}{l}\text { Short-term loss to follow-up based on pri- } \\
\text { mary depression outcome (HSCL } \geq 50 \%) \\
\text { was: overall } 26 / 354(7 \%), 12 / 168(7 \%) \text { in- } \\
\text { tervention and } 14 / 186(8 \%) \text { control. Rea- } \\
\text { sons for loss to follow-up not provided. } \\
\text { Used intention-to-treat analysis }\end{array}$ \\
\hline Selective reporting (reporting bias) & Unclear risk & Insufficient information available to assess \\
\hline Other bias & Unclear risk & Insufficient information available to assess \\
\hline Implementation Integrity & Unclear risk & Insufficient information available to assess \\
\hline $\begin{array}{l}\text { Blinding of participants and personnel } \\
\text { (performance bias) } \\
\text { All outcomes }\end{array}$ & High risk & $\begin{array}{l}\text { Participants and personnel could not be } \\
\text { blinded, outcome likely to be influenced by } \\
\text { lack of blinding }\end{array}$ \\
\hline $\begin{array}{l}\text { Blinding of outcome assessment (detection } \\
\text { bias) } \\
\text { All outcomes }\end{array}$ & Low risk & $\begin{array}{l}\text { Assessor was not aware of treatment alloca- } \\
\text { tion }\end{array}$ \\
\hline
\end{tabular}

Hilty 2007

\begin{tabular}{|c|c|}
\hline Methods & Study design: Randomised controlled trial \\
\hline Participants & $\begin{array}{l}\text { Setting: Primary care } \\
\text { Diagnosis: Major depression assessed using a structured diagnostic interview (i.e. mood } \\
\text { and psychotic sections of the Structured Clinical Interview for DSM-IV [SCID-I, re- } \\
\text { search version] and self-report measures - Beck Depression Inventory (BDI-13), Symp- } \\
\text { tom Checklist-90 Revised (HSCL-90-R), Medical Outcomes Study Short Form } 36 \text { (SF- } \\
\text { 36) } \\
\text { Inclusion criteria: Rural primary care. Subjects were English-speaking men and women, } \\
\text { between ages of } 18 \text { and } 80 \text { years, who were willing to take an antidepressant medication } \\
\text { Exclusion criteria: Bipolar, schizoaffective, and schizophrenic disorders, no primary di- } \\
\text { agnosis of major depression, suicidal intention or plans. Patients with dementia, preg- } \\
\text { nancy, terminal illness, and plans to move in the next } 12 \text { months were not enrolled } \\
\text { Age: Mean } 46 \text { years } \\
\text { Gender: } 80 \% \text { female } \\
\text { Ethnicity: } 90 \% \text { caucasian } \\
\text { Country: United States } \\
\text { Sample size (randomised): Total participants } 93 \text {, intervention 52, control } 41\end{array}$ \\
\hline
\end{tabular}

Interventions

Intervention: Intensive Disease Management Module

Contains the four elements of collaborative care:

1) a multi-professional approach to patient care: Primary care physician (PCP), study nurse or investigator $(\mathrm{CM})$, psychiatrist (MH specialist)

2) a structured management plan: Patients received a handout and a video on the biology of depression and how ADs work and had 5 scheduled PCP visits in 16 weeks. CM 
Hilty 2007 (Continued)

telephoned patients to assess adherence and side effects of medication, with referral to the PCP or MH specialist if needed and proactive follow-up for missed appointments. Five telepsychiatric consultations were offered in 18 weeks

3) scheduled patient follow-ups: CM telephone calls at 2 and 4 weeks

4) enhanced inter-professional communication: PCP and the telepsychiatrist discussed cases by telephone or via televideo after each telepsychiatric consultation. The MH specialist trained the PCP to administer care in accordance to national guidelines. PCP contacted the $\mathrm{MH}$ specialist by telephone regarding questions. For urgent issues the study coordinator notified the MH specialist, CM and PCP

Control: Disease Management Module and some patients received a one-off telepsychiatry visit as per pre-trial standard

\begin{tabular}{ll} 
Outcomes & $\begin{array}{l}\text { Depression (BDI-13): 3, 6, } 12 \text { months } \\
\text { Quality of Life (mental and physical health): 3, 6, } 12 \text { months } \\
\text { Satisfaction: 3, 6, 12 months }\end{array}$ \\
\hline Notes & $\begin{array}{l}\text { BDI: Beck Depression Inventory; CM: case manager; DSM-IV: Diagnostic and Statis- } \\
\text { tical Manual fourth edition; HSCL: Hopkins Sympton Checklist; MH: mental health; } \\
\text { PCP: primary care provider }\end{array}$
\end{tabular}

\section{Risk of bias}

\begin{tabular}{|c|c|c|}
\hline Bias & Authors' judgement & Support for judgement \\
\hline $\begin{array}{l}\text { Random sequence generation (selection } \\
\text { bias) }\end{array}$ & Low risk & Random table of numbers \\
\hline Allocation concealment (selection bias) & Low risk & $\begin{array}{l}\text { Allocation conducted by an individual not } \\
\text { involved in patient recruitment }\end{array}$ \\
\hline $\begin{array}{l}\text { Incomplete outcome data (attrition bias) } \\
\text { All outcomes }\end{array}$ & Unclear risk & $\begin{array}{l}\text { Short-term loss to follow-up based on pri- } \\
\text { mary depression outcome (BDI) was un- } \\
\text { clear. Reasons for loss to follow-up not pro- } \\
\text { vided. Used intention-to-treat analysis }\end{array}$ \\
\hline Selective reporting (reporting bias) & Unclear risk & Insufficient information available to assess \\
\hline Other bias & Unclear risk & Insufficient information available to assess \\
\hline Implementation Integrity & Unclear risk & Insufficient information available to assess \\
\hline $\begin{array}{l}\text { Blinding of participants and personnel } \\
\text { (performance bias) } \\
\text { All outcomes }\end{array}$ & High risk & $\begin{array}{l}\text { Participants and personnel could not be } \\
\text { blinded, outcome likely to be influenced by } \\
\text { lack of blinding }\end{array}$ \\
\hline $\begin{array}{l}\text { Blinding of outcome assessment (detection } \\
\text { bias) } \\
\text { All outcomes }\end{array}$ & Unclear risk & Insufficient information available to assess \\
\hline
\end{tabular}

Collaborative care for depression and anxiety problems (Review) 
Methods

Participants
Study design: Randomised controlled trial

Setting: Specialist, primary care

Diagnosis: Clinical depression defined as a PHQ-9 score greater than or equal to 10, with 5 or more symptoms (including either depressed mood or anhedonia) present more than half the days for at least the preceding 2 weeks

Inclusion criteria: Hospitalised cardiac patients. Eligible patients were admitted to 1 of 3 inpatient cardiac units at an urban academic medical centre for acute cardiac disease, defined as admission for myocardial infarction, unstable angina, decompensated heart failure, or arrhythmia

Exclusion criteria: Bipolar disorder, psychotic symptoms, active substance abuse, active suicidal ideation, unable to speak English or unable to provide informed consent due to cognitive problems or the severity of their current medical illness

Age: Mean 62.4 years

Gender: $49 \%$ female

Ethnicity: 92\% white

Country: United States

Sample size (randomised): Total participants 175, intervention 90, control 85
Intervention: Collaborative care

Contains the four elements of collaborative care:

1) a multi-professional approach to patient care: Primary medical physician including cardiologist (PCP), social worker (CM), psychiatrist (MH specialist)

2) a structured management plan: In hospital interventions: CM provided written and verbal education about depression and its impact on cardiac disease, helped the patient to schedule pleasurable activities after discharge, and described treatment options (pharmacotherapy or psychotherapy referral). CM then consulted with $\mathrm{MH}$ specialist, who developed individualised depression treatment recommendations based on previous/current treatment and preference including SSRI and/or referral for therapy. CM worked to coordinate these recommendations with inpatient and outpatient medical care providers. Post-discharge interventions: Post-discharge interventions lasted 12 weeks. For patients with clinical depression at any follow-up, a multi-component intervention (similar to the in-hospital intervention) was undertaken. CM discussed the case with the $\mathrm{MH}$ specialist, written treatment recommendations were generated (e.g., increase $\mathrm{AD}$, therapy referral) . These were discussed with the patient and the PCP (and faxed to the PCP), and the CM worked to coordinate implementation

3) scheduled patient follow-ups: Inpatient = unclear, post-discharge $=3$ telephone calls in 12 weeks

4) enhanced inter-professional communication: Treatment recommendations discussed with and faxed to PCP. CM and MH specialists held weekly team meetings

Control: Treatment as usual enhanced as CM informed the inpatient treatment team of the patient's depression and recommended that the patient receive treatment. If patients met criteria for clinical depression at follow-up, the PCP was informed via written communication that the patient had ongoing depression and would benefit from treatment

Outcomes

Depression (PHQ-9): 6, 12 months

Medication use: 6 weeks

Quality of Life (mental and physical health): 6 weeks, 3, 6 months

Satisfaction: 6 months 
Huffman 2011 (Continued)

\begin{tabular}{|c|c|c|}
\hline Notes & \multicolumn{2}{|c|}{$\begin{array}{l}\text { CM: case manager; MH: mental health; PCP: primary care provider; PHQ-9: Patient } \\
\text { Health Questionnaire }\end{array}$} \\
\hline \multicolumn{3}{|l|}{ Risk of bias } \\
\hline Bias & Authors' judgement & Support for judgement \\
\hline $\begin{array}{l}\text { Random sequence generation (selection } \\
\text { bias) }\end{array}$ & Low risk & Random number generator \\
\hline Allocation concealment (selection bias) & Unclear risk & Insufficient information available to assess \\
\hline $\begin{array}{l}\text { Incomplete outcome data (attrition bias) } \\
\text { All outcomes }\end{array}$ & Unclear risk & $\begin{array}{l}\text { Short-term loss to follow-up based on pri- } \\
\text { mary depression outcome (PHQ- } 950 \% \\
\text { decrease) was: overall } 37 / 175 \text { ( } 21 \%), 19 / \\
90(21 \%) \text { intervention and } 18 / 85(21 \%) \\
\text { control. Reasons for loss to follow-up not } \\
\text { reported by group. Intention-to-treat anal- } \\
\text { ysis reported using random-effects regres- } \\
\text { sion models to manage missing data }\end{array}$ \\
\hline Selective reporting (reporting bias) & Unclear risk & Insufficient information available to assess \\
\hline Other bias & Unclear risk & Insufficient information available to assess \\
\hline Implementation Integrity & Unclear risk & Insufficient information available to assess \\
\hline $\begin{array}{l}\text { Blinding of participants and personnel } \\
\text { (performance bias) } \\
\text { All outcomes }\end{array}$ & High risk & $\begin{array}{l}\text { Participants and personnel could not be } \\
\text { blinded, outcome likely to be influenced by } \\
\text { lack of blinding }\end{array}$ \\
\hline $\begin{array}{l}\text { Blinding of outcome assessment (detection } \\
\text { bias) } \\
\text { All outcomes }\end{array}$ & Low risk & $\begin{array}{l}\text { Assessor was not aware of treatment alloca- } \\
\text { tion }\end{array}$ \\
\hline
\end{tabular}

Hunkeler 2000

Methods

Participants
Study design: Randomised controlled trial

Setting: Primary care

Diagnosis: Diagnosed by PCP as having major depressive disorder or dysthymia and given a prescription for a selective serotonin reuptake inhibitor (SSRI) antidepressant Inclusion criteria: Major depressive disorder or dythymia as diagnosed by PCP

Exclusion criteria: Had received a previous antidepressant drug prescription within the past 6 months, had an inadequate command of the English language, reported current problems with substance abuse, showed current suicide risk, or reported thoughts of violence, required referral to other treatment, currently receiving an anti-depressant drug or psychotherapy or refusing prescribed SSRI 
Hunkeler 2000 (Continued)

Age: Mean 55.4 years

Gender: 69\% female

Ethnicity: 63\% white

Country: United States

Sample size (randomised): Total participants 302, intervention 179, control 123

Interventions

Intervention: Nurse telehealthcare (with or without peer support)

Contains the four elements of collaborative care:

1) a multi-professional approach to patient care: Primary care physician (PCP), nurse (CM), psychologist (MH specialist)

2) a structured management plan: During telephone calls CM inquired about medication, managed minor side effects, and emphasised the importance of taking medication regularly. CMs also offered emotional support and helped patients identify active and pleasurable activities reviewing activities of the previous week and developing an action plan with the patient. Peer support was provided by trained volunteer health plan members who had experienced a successfully treated episode of major depression or dysthymia. Peers contacted patients by telephone or visited them at least once and continued support over 6 months

3) scheduled patient follow-ups: 12 to 14 telephone calls in 16 weeks, 1 to 2 per week for first 2 weeks, 1 per week in weeks 3 to 8 and then fortnightly up to week 16

4) enhanced inter-professional communication: CMs gave regular feedback on the progress of each patient to the patient's PCP. CMs received weekly telephone and monthly visits for supervision from $\mathrm{MH}$ specialist

Control: Treatment as usual enhanced as PCPs received training on assessment and treatment of depression

Depression (BDI): 6 weeks, 6 months
Medication use: 6 weeks, 6 months
Quality of Life (mental and physical health): 6 weeks, 6 months
Satisfaction: 6 weeks, 6 months

Notes

BDI: Beck Depression Inventory; CM: case manager; $\mathrm{MH}$ : mental health; PCP: primary care provider

\section{Risk of bias}

Bias Authors' judgement

Random sequence generation (selection Unclear risk bias)

\begin{tabular}{|c|c|c|}
\hline Allocation concealment (selection bias) & Unclear risk & Insufficient information available to assess \\
\hline $\begin{array}{l}\text { Incomplete outcome data (attrition bias) } \\
\text { All outcomes }\end{array}$ & Unclear risk & $\begin{array}{l}\text { Short-term loss to follow-up based on pri- } \\
\text { mary depression outcome (BDI } 50 \% \text { im- } \\
\text { provement) was: overall } 47 / 302(16 \%), 29 / \\
179(16 \%) \text { intervention and } 18 / 123(15 \%) \\
\text { control. Reasons for loss to follow-up not } \\
\text { provided by group. Intention to treat not }\end{array}$ \\
\hline
\end{tabular}

\section{Support for judgement}

Insufficient information available to assess

Collaborative care for depression and anxiety problems (Review)

Copyright $\odot 2012$ The Cochrane Collaboration. Published by John Wiley \& Sons, Ltd. 
Hunkeler 2000 (Continued)

reported

\begin{tabular}{l|ll}
\hline Selective reporting (reporting bias) & Unclear risk & Insufficient information available to assess \\
\hline Other bias & Unclear risk & Insufficient information available to assess \\
\hline $\begin{array}{l}\text { Implementation Integrity } \\
\text { Blinding of participants and personnel } \\
\begin{array}{l}\text { (performance bias) } \\
\text { All outcomes }\end{array}\end{array}$ & High risk & Insufficient information available to assess \\
\hline $\begin{array}{l}\text { Blinding of outcome assessment (detection } \\
\text { bias) } \\
\text { All outcomes }\end{array}$ & Unclear risk & $\begin{array}{l}\text { Participants and personnel could not be } \\
\text { blinded, outcome likely to be influenced by } \\
\text { lack of blinding }\end{array}$ \\
\hline
\end{tabular}

Katon 1995a

\begin{tabular}{|c|c|}
\hline Methods & Study design: Randomised controlled trial \\
\hline Participants & $\begin{array}{l}\text { Setting: Primary care } \\
\text { Diagnosis: Diagnosed by the primary care physician as meeting criteria for definite or } \\
\text { probable major depression and who had agreed to antidepressant therapy. A 20-item } \\
\text { Symptom Checklist (HSCL) depression screening score of } 0.75 \text { or greater } \\
\text { Inclusion criteria: Aged between } 18 \text { and } 80 \text { years and willingness to take antidepressant } \\
\text { medication } \\
\text { Exclusion criteria: Current alcohol abuse, current psychotic symptoms, serious suicidal } \\
\text { ideation or plan, dementia, pregnancy, terminal illness, limited command of English; } \\
\text { and plan to disenroll from the insurance plan within the next } 12 \text { months } \\
\text { Age: Mean } 51.3 \text { years } \\
\text { Gender: } 73 \% \text { female } \\
\text { Ethnicity: Not stated } \\
\text { Country: United States } \\
\text { Sample size (randomised: Minor depression): Total participants 126, intervention 59, } \\
\text { control } 67\end{array}$ \\
\hline Interventions & $\begin{array}{l}\text { Intervention: Multifaceted intervention programme } \\
\text { Contains the four elements of collaborative care: } \\
\text { 1) a multi-professional approach to patient care: Primary care physician (PCP), psychi- } \\
\text { atrist (CM/MH specialist) } \\
\text { 2) a structured management plan: Patients received a brief booklet on the biology of } \\
\text { depression, the mechanism of action of ADs, a second booklet on simple CBT techniques } \\
\text { and a videotape covering similar material. Patients were asked to write down any questions } \\
\text { in preparation for their initial meeting. The CM educated the patient about depression } \\
\text { and ADs and side-effects and also reviewed stressful life events. Changes in ADs could be } \\
\text { initiated by PCP or CM after verbal consultation. CM monitored automatic pharmacy } \\
\text { data to assess adherence } \\
\text { 3) scheduled patient follow-ups: } 2-4 \text { visits over } 4 \text { to } 6 \text { weeks interspersed with } 2 \text { scheduled }\end{array}$ \\
\hline
\end{tabular}




$\begin{array}{ll} & \text { visits with PCP 7-10 days apart } \\ \text { 4) enhanced inter-professional communication: CMs and PCPs held monthly case con- } \\ \text { ferences and case by case consultations. CMs helped PCPs choose alternative medication } \\ \text { for patients. Change in ADs could be initiated by the PCP or CM after verbal consulta- } \\ \text { tion. PCPs received a typed psychiatric consultation note within one week. CMs notified } \\ \text { PCPs when patients failed to refill AD prescriptions } \\ \text { Control: Treatment as usual }\end{array}$

Notes

CM: case manager; HSCL: Hopkins Sympton Checklist; MH: mental health; PCP: primary care provider

\section{Risk of bias}

\section{Bias}

Random sequence generation (selection Low risk bias)

\begin{tabular}{|c|c|c|}
\hline Allocation concealment (selection bias) & Unclear risk & Insufficient information available to assess \\
\hline $\begin{array}{l}\text { Incomplete outcome data (attrition bias) } \\
\text { All outcomes }\end{array}$ & Unclear risk & $\begin{array}{l}\text { Short-term loss to follow-up based on pri- } \\
\text { mary depression outcome (HSCL } 50 \% \text { im- } \\
\text { provement) was: overall 13/126 (10\%), 6/ } \\
59(10 \%) \text { intervention and } 7 / 67(10 \%) \\
\text { control. Reasons for loss to follow-up not } \\
\text { provided. Used intention-to-treat analysis }\end{array}$ \\
\hline Selective reporting (reporting bias) & Unclear risk & Insufficient information available to assess \\
\hline Other bias & High risk & $\begin{array}{l}\text { Reported analysis not by randomised group } \\
\text { but by participants with a) minor and b) } \\
\text { major depression }\end{array}$ \\
\hline Implementation Integrity & Unclear risk & Insufficient information available to assess \\
\hline $\begin{array}{l}\text { Blinding of participants and personnel } \\
\text { (performance bias) } \\
\text { All outcomes }\end{array}$ & High risk & $\begin{array}{l}\text { Participants and personnel could not be } \\
\text { blinded, outcome likely to be influenced by } \\
\text { lack of blinding }\end{array}$ \\
\hline $\begin{array}{l}\text { Blinding of outcome assessment (detection } \\
\text { bias) } \\
\text { All outcomes }\end{array}$ & Low risk & $\begin{array}{l}\text { Assessor was not aware of treatment alloca- } \\
\text { tion }\end{array}$ \\
\hline
\end{tabular}

\section{Support for judgement}

Computer generated in blocks of 8 ss 
Methods

Participants

Interventions
Study design: Randomised controlled trial

Setting: Primary care

Diagnosis: Diagnosed by the primary care physician as meeting criteria for definite or probable major depression and who had agreed to antidepressant therapy. Symptom Checklist (HSCL-20) depression screening score of 0.75 or greater

Inclusion criteria: Aged between 18 and 80 years and willingness to take antidepressant medication

Exclusion criteria: Current alcohol abuse, current psychotic symptoms, serious suicidal ideation or plan, dementia, pregnancy, terminal illness, limited command of English; and plan to disenroll from the insurance plan within the next 12 months

Age: Mean 42.8 years

Gender: $83 \%$ female

Ethnicity: Not stated

Country: United States

Sample size (randomised: Major depression): Total participants 91, intervention 49, control 42

Intervention: Multifaceted intervention programme

Contains the four elements of collaborative care:

1) a multi-professional approach to patient care: Primary care physician (PCP), psychiatrist (CM/MH specialist)

2) a structured management plan: Patients received a brief booklet on the biology of depression, the mechanism of action of ADs, a second booklet on simple CBT techniques and a videotape covering similar material. Patients were asked to write down any questions in preparation for their initial meeting. The CM educated the patient about depression and $\mathrm{ADs}$ and side-effects and also reviewed stressful life events. Changes in ADs could be initiated by PCP or CM after verbal consultation. CM monitored automatic pharmacy data to assess adherence

3) scheduled patient follow-ups: 2-4 visits over 4 to 6 weeks interspersed with 2 scheduled visits with PCP 7-10 days apart

4) enhanced inter-professional communication: CMs and PCPs held monthly case conferences and case by case consultations. CMs helped PCPs choose alternative medication for patients. Change in ADs could be initiated by the PCP or CM after verbal consultation. PCPs received a typed psychiatric consultation note within one week. CMs notified $\mathrm{PCPs}$ when patients failed to refill $\mathrm{AD}$ prescriptions

Control: Treatment as usual

Outcomes

Depression (HSCL): 4 months

Medication use: 1, 4 months

Satisfaction: 4 months

Notes

AD: antidepressant; CM: case manager; HSCL: Hopkins Symptom Checklist; MH: mental health; PCP: primary care provider

Risk of bias

Bias

Authors' judgement
Support for judgement 
Katon 1995b (Continued)

\begin{tabular}{|c|c|c|}
\hline $\begin{array}{l}\text { Random sequence generation (selection } \\
\text { bias) }\end{array}$ & Low risk & Computer generated in blocks of 8 \\
\hline Allocation concealment (selection bias) & Unclear risk & Insufficient information available to assess \\
\hline $\begin{array}{l}\text { Incomplete outcome data (attrition bias) } \\
\text { All outcomes }\end{array}$ & Unclear risk & $\begin{array}{l}\text { Short-term loss to follow-up based on pri- } \\
\text { mary depression outcome (HSCL } 50 \% \text { im- } \\
\text { provement) was: overall 10/91 (11\%), 5/49 } \\
\text { (10\%) intervention and } 5 / 42(12 \%) \text { con- } \\
\text { trol. Reasons for loss to follow-up not pro- } \\
\text { vided. Used intention-to-treat analysis }\end{array}$ \\
\hline Selective reporting (reporting bias) & Unclear risk & Insufficient information available to assess \\
\hline Other bias & High risk & $\begin{array}{l}\text { Reported analysis not by randomised group } \\
\text { but by participants with a) minor and b) } \\
\text { major depression }\end{array}$ \\
\hline Implementation Integrity & Unclear risk & Insufficient information available to assess \\
\hline $\begin{array}{l}\text { Blinding of participants and personnel } \\
\text { (performance bias) } \\
\text { All outcomes }\end{array}$ & High risk & $\begin{array}{l}\text { Participants and personnel could not be } \\
\text { blinded, outcome likely to be influenced by } \\
\text { lack of blinding }\end{array}$ \\
\hline
\end{tabular}

Blinding of outcome assessment (detection Low risk bias)

Assessor was not aware of treatment allocation

All outcomes

Katon 1996a

\begin{tabular}{ll} 
Methods & Study design: Randomised controlled trial \\
\hline Participants & Setting: Primary care \\
Diagnosis: Diagnosed by Primary Care Physician as meeting criteria for definite or \\
probable major depression and who scored 0.75 or greater on the 20 -item depression \\
symptom checklist (HSCL-20). Stratified into moderate and severe depression groups \\
based on their HSCL-20 score (moderate, 0.75 to $<1.75$, severe, 1.75 to 4.0$)$ \\
Inclusion criteria: Aged 18 to 80 years, willingness to take antidepressant medication \\
Exclusion criteria: Current alcohol abuse, current psychotic symptoms, serious suicidal \\
ideation or plan, dementia, pregnancy, terminal illness, limited command of English and \\
plan to withdraw from the insurance plan within the next 12 months \\
Age: Mean 48.2 years \\
Gender: $73 \%$ female \\
Ethnicity: Not stated \\
Country: United States \\
Sample size (randomised: Minor depression): Total participants 88, intervention 46, \\
control 42
\end{tabular}


Interventions
Intervention: Multifaceted intervention programme

Contains the four elements of collaborative care:

1) a multi-professional approach to patient care: Primary care provider (PCP), psychologist (CM), psychiatrist (MH specialist)

2) a structured management plan: Programme involved teaching cognitive behavioural skills to manage depression and counselling to improve medication adherence. Sessions 1-4 involved education, skills training, and homework assignments or behavioural experiments. Optional sessions ( $5 \& 6$ ) involved skills training, problem-solving, and relaxation training. A relapse prevention plan was also developed. Patients received a brief booklet on the biology of depression, the mechanism of action of ADs, a second booklet on simple CBT techniques and a videotape covering similar material. Non-response patients could be referred to the $\mathrm{MH}$ specialist for direct visit

3) scheduled patient follow-ups: 4-6 contacts within 6 weeks and 4 telephone calls at 2, 4, 12 and 24 weeks

4) enhanced inter-professional communication: Case-by-case consultation occurred between CM and PCP and CM's provided PCPs with a handwritten consultation note after each patient contact. A copy of the relapse prevention plan was put in the patient's medical notes. $\mathrm{CM}$ and $\mathrm{MH}$ specialist met weekly for supervision and CM recommended medication changes (which had been made by the MH specialist) to the PCP Control: Treatment as usual

Outcomes

Depression (HSCL): 4 months

Medication use: 1, 4, 7 months

Satisfaction: 4 months

Notes

AD: antidepressant; CM: case manager; HSCL: Hopkins Symptom Checklist; MH: mental health; PCP: primary care provider

\section{Risk of bias}

\begin{tabular}{|c|c|c|}
\hline Bias & Authors' judgement & Support for judgement \\
\hline $\begin{array}{l}\text { Random sequence generation (selection } \\
\text { bias) }\end{array}$ & Low risk & Computer generated in blocks of 8 \\
\hline Allocation concealment (selection bias) & Unclear risk & Insufficient information available to assess \\
\hline $\begin{array}{l}\text { Incomplete outcome data (attrition bias) } \\
\text { All outcomes }\end{array}$ & Unclear risk & $\begin{array}{l}\text { Short-term loss to follow-up based on pri- } \\
\text { mary depression outcome (HSCL 50\% im- } \\
\text { provement) was: overall 14/88 (16\%), 7/46 } \\
\text { (15\%) intervention and 7/42 (17\%) con- } \\
\text { trol. Reasons for loss to follow-up not pro- } \\
\text { vided. Used intention-to-treat analysis }\end{array}$ \\
\hline Selective reporting (reporting bias) & Unclear risk & Insufficient information available to assess \\
\hline Other bias & High risk & $\begin{array}{l}\text { Reported analysis not by randomised group } \\
\text { but by participants with a) minor and b) }\end{array}$ \\
\hline
\end{tabular}


major depression

\begin{tabular}{|c|c|c|}
\hline Implementation Integrity & Low risk & $\begin{array}{l}\text { Attempts were made to assess implemen- } \\
\text { tation integrity (e.g. direct observation or } \\
\text { rating of tapes) }\end{array}$ \\
\hline
\end{tabular}

Blinding of participants and personnel High risk (performance bias)

All outcomes

Blinding of outcome assessment (detection Low risk bias)

All outcomes
Participants and personnel could not be blinded, outcome likely to be influenced by lack of blinding

Assessor was not aware of treatment allocation

Katon 1996b

Methods

Participants

Interventions
Study design: Randomised controlled trial

Setting: Primary care

Diagnosis: Diagnosed by PCP as meeting criteria for definite or probable major depression and who scored 0.75 or greater on the Symptom Checklist (HSCL-20). Stratified into moderate and severe depression groups based on their HSCL-20 score (moderate, 0.75 to $<1.75$, severe, 1.75 to 4.0 )

Inclusion criteria: Aged 18 to 80 years, willingness to take antidepressant medication

Exclusion criteria: Current alcohol abuse, current psychotic symptoms, serious suicidal ideation or plan, dementia, pregnancy, terminal illness, limited command of English and plan to withdraw from the insurance plan within the next 12 months

Age: Mean 44 years

Gender: $75 \%$ female

Ethnicity: Not stated

Country: United States

Sample size (randomised: Major depression): Total participants 65, intervention 31, control 34

Intervention: Multifaceted intervention programme

Contains the four elements of collaborative care:

1) a multi-professional approach to patient care: Primary care provider (PCP), psychologist $(\mathrm{CM})$, psychiatrist (MH specialist)

2) a structured management plan: Programme involved teaching cognitive behavioural skills to manage depression and counselling to improve medication adherence. Sessions 1-4 involved education, skills training, and homework assignments or behavioural experiments. Optional sessions ( $5 \& 6$ ) involved skills training, problem-solving, and relaxation training. A relapse prevention plan was also developed. Patients received a brief booklet on the biology of depression, the mechanism of action of ADs, a second booklet on simple CBT techniques and a videotape covering similar material. Non-response patients could be referred to the $\mathrm{MH}$ specialist for direct visit

3) scheduled patient follow-ups: 4-6 contacts within 6 weeks and 4 telephone calls at 2,

4, 12 and 24 weeks 
4) enhanced inter-professional communication: Case-by-case consultation occurred between CM and PCP and CM's provided PCPs with a handwritten consultation note after each patient contact. A copy of the relapse prevention plan was put in the patient's medical notes. CM and MH specialist met weekly for supervision and CM recommended medication changes (which had been made by the MH specialist) to the PCP Control: Treatment as usual

\begin{tabular}{ll} 
Outcomes & $\begin{array}{l}\text { Depression (HSCL): } 4 \text { months } \\
\text { Medication use: } 1,4,7 \text { months } \\
\text { Satisfaction: } 4 \text { months }\end{array}$ \\
\hline Notes & AD: antidepressant; CM: case manager; HSCL: Hopkins Symptom Checklist; MH: \\
mental health; PCP: primary care provider
\end{tabular}

\section{Risk of bias}

\begin{tabular}{|c|c|c|}
\hline Bias & Authors' judgement & Support for judgement \\
\hline $\begin{array}{l}\text { Random sequence generation (selection } \\
\text { bias) }\end{array}$ & Low risk & Computer generated in blocks of 8 \\
\hline Allocation concealment (selection bias) & Unclear risk & Insufficient information available to assess \\
\hline $\begin{array}{l}\text { Incomplete outcome data (attrition bias) } \\
\text { All outcomes }\end{array}$ & Unclear risk & $\begin{array}{l}\text { Short-term loss to follow-up based on pri- } \\
\text { mary depression outcome (HSCL } 50 \% \text { im- } \\
\text { provement) was: overall } 10 / 65(15 \%), 5 / 31 \\
(16 \%) \text { intervention and } 5 / 34(15 \%) \text { con- } \\
\text { trol. Reasons for loss to follow-up not pro- } \\
\text { vided. Used intention-to-treat analysis }\end{array}$ \\
\hline Selective reporting (reporting bias) & Unclear risk & Insufficient information available to assess \\
\hline Other bias & High risk & $\begin{array}{l}\text { Reported analysis not by randomised group } \\
\text { but by participants with a) minor and b) } \\
\text { major depression }\end{array}$ \\
\hline Implementation Integrity & Low risk & $\begin{array}{l}\text { Attempts were made to assess implemen- } \\
\text { tation integrity (e.g. direct observation or } \\
\text { rating of tapes) }\end{array}$ \\
\hline $\begin{array}{l}\text { Blinding of participants and personnel } \\
\text { (performance bias) } \\
\text { All outcomes }\end{array}$ & High risk & $\begin{array}{l}\text { Participants and personnel could not be } \\
\text { blinded, outcome likely to be influenced by } \\
\text { lack of blinding }\end{array}$ \\
\hline $\begin{array}{l}\text { Blinding of outcome assessment (detection } \\
\text { bias) } \\
\text { All outcomes }\end{array}$ & Low risk & $\begin{array}{l}\text { Assessor was not aware of treatment alloca- } \\
\text { tion }\end{array}$ \\
\hline
\end{tabular}


Methods

Participants
Study design: Randomised controlled trial

Setting: Primary care

Diagnosis: Diagnosis of depression or anxiety and patients at high risk for persistent depression. The first-stage screen included the telephone Structured Clinical Interview for DSM-III-R (SCID). Criteria for selection for the second-stage interview were having 4 or more residual major depressive symptoms, recurrent depression (2 or more prior episodes), or dysthymia. Four or more major depressive symptoms on the SCID and a score of 1.0 or greater on the 20 depression items of the Hopkins Symptom Checklist (HSCL-20) or having fewer than 4 DSM-IV major depressive symptoms but with a score of 1.5 or greater on the HSCL-20.16

Inclusion criteria: Patients between the ages of 18 and 80 years who received a new antidepressant prescription (no prior prescriptions within the last 120 days) from a primary care physician

Exclusion criteria: A screening score of 2 or more on the CAGE alcohol screening questionnaire, being pregnant or currently nursing, planning to pull out from the Group Health Cooperative insurance plan within the next 12 months, currently seeing a psychiatrist, having limited command of English, and recently using lithium or antipsychotic medication

Age: Mean 47 (SD 13.7) years

Gender: $75 \%$ female

Ethnicity: $80 \%$ White

Country: United States

Sample size (randomised): Total participants 228, intervention 114, control 114

Interventions

Intervention: Stepped collaborative care

Contains the four elements of collaborative care:

1) a multi-professional approach to patient care: Primary care provider (PCP), psychiatrist (CM/MH specialist)

2) a structured management plan: All patients were prescribed an AD 8-9 weeks before initial intervention visit in which $\mathrm{CM}$ assessed clinical status and current medication adherence and side effects. CM helped the patient and PCP alter AD medication and monitored medication adherence by checking automated pharmacy data and alerted the PCP if premature discontinuation occurred. CMs also referred patients with severe psychosocial stressors for psychotherapy or support groups

3) scheduled patient follow-ups: 2 in 4 weeks with 2 additional if non-response with a brief telephone call in between ( 2 calls in total)

4) enhanced inter-professional communication: CM informed PCP of non-adherence. After final visit, the CM sent PCP a standardised note of the AD prescribed, recommended duration of treatment, residual depressive symptoms and recommendations for therapy

Control: Treatment as usual

Depression (SCID): 3, 6 months

Medication use: 1, 3, 6 months

Quality of Life (mental and physical health): 1, 3, 6 months

Satisfaction: 3, 6 months

AD: antidepressant; CM: case manager; HSCL: Hopkins Symptom Checklist; MH: mental health; PCP: primary care provider 
Katon 1999 (Continued)

\section{Risk of bias}

\begin{tabular}{|c|c|c|}
\hline Bias & Authors' judgement & Support for judgement \\
\hline $\begin{array}{l}\text { Random sequence generation (selection } \\
\text { bias) }\end{array}$ & Low risk & Computer generated in blocks of 8 \\
\hline Allocation concealment (selection bias) & Unclear risk & Insufficient information available to assess \\
\hline $\begin{array}{l}\text { Incomplete outcome data (attrition bias) } \\
\text { All outcomes }\end{array}$ & Unclear risk & $\begin{array}{l}\text { Short-term loss to follow-up based on pri- } \\
\text { mary depression outcome (Asymptomatic } \\
\text { by SCID) was: overall 36/228(16\%), } 18 / \\
114(16 \%) \text { intervention and } 18 / 114(16 \%) \\
\text { control. Reasons for loss to follow-up not } \\
\text { provided. Used intention-to-treat analysis }\end{array}$ \\
\hline Selective reporting (reporting bias) & Unclear risk & Insufficient information available to assess \\
\hline Other bias & Unclear risk & Insufficient information available to assess \\
\hline Implementation Integrity & Unclear risk & Insufficient information available to assess \\
\hline $\begin{array}{l}\text { Blinding of participants and personnel } \\
\text { (performance bias) } \\
\text { All outcomes }\end{array}$ & High risk & $\begin{array}{l}\text { Participants and personnel could not be } \\
\text { blinded, outcome likely to be influenced by } \\
\text { lack of blinding }\end{array}$ \\
\hline $\begin{array}{l}\text { Blinding of outcome assessment (detection } \\
\text { bias) } \\
\text { All outcomes }\end{array}$ & Low risk & $\begin{array}{l}\text { Assessor was not aware of treatment alloca- } \\
\text { tion }\end{array}$ \\
\hline
\end{tabular}

Katon 2001

Methods Study design: Randomised controlled trial

Participants Setting: Primary care

Diagnosis: Diagnosis of depression or anxiety and patients at high risk of relapse. The first-stage screen included the depression section of the telephone Structured Clinical Interview for DSM-III-R (SCID), Selection criteria for the second stage interview were either having a high epidemiologic risk of relapse or 4 or more residual major depressive symptoms. Fewer than 4 DSM-IV major depressive symptoms and a history of 3 or more episodes of major depression or dysthymia or 4 residual depressive symptoms but with a mean Symptom Checklist (HSCL-20) depression score of less than 1.0 and a history of major depression/dysthymia

Inclusion criteria: Patients between the ages of 18 and 80 years from 1 of 4 primary care clinics who received a new antidepressant prescription (no prior prescriptions within the last 120 days) from a primary care physician

Exclusion criteria: Screening score of 2 or more on the CAGE alcohol screening questionnaire, being pregnant or currently nursing, planning to disenroll from insurance plan 
within the next 12 months, currently seeing a psychiatrist, having limited command of English, and recently using lithium or antipsychotic medication

Age: Mean 46 years

Gender: $74 \%$ female

Ethnicity: 90\% White

Country: United States

Sample size (randomised): Total participants 386, intervention 194, control 192

Interventions

Intervention: Relapse prevention programme

Contains the four elements of collaborative care:

1) a multi-professional approach to patient care: Primary care provider (PCP), psychologist, nurse, social worker (CM), psychiatrist ( $\mathrm{MH}$ specialist)

2) a structured management plan: Patients were provided a book and videotape aimed at increasing patient education and enhancing self-treatment of their depression. CM assessed clinical status and biopsychosocial history. The intervention aimed to improve long-term adherence to $\mathrm{ADs}$, increase self-monitoring and relapse prevention strategies such as early help seeking. Other goals were increasing pleasant activities, exercise, and socializing, and identifying potential high-risk situations to promote problem-solving ability, coping, and self-efficacy for managing depression. Follow-up telephone calls and personalised mailings monitored progress and adherence to the plan

3 ) scheduled patient follow-ups: 2 visits and telephone calls at 1, 4 and 8.5 months after session 2. Personalised mailings at 2, 6, 10 and 12 months

4) enhanced inter-professional communication: PCPs received intermittent verbal and written consultation about patient progress and a copy of the relapse prevention plan. CMs had weekly supervision with MH specialists

Control: Treatment as usual enhanced as PCPs were notified of group allocation

Outcomes Depression (HSCL): 3, 6, 9, 12 months

Medication use: 3, 6, 9, 12 months

Quality of Life (mental and physical health): 3, 6, 9, 12 months

Notes

AD: antidepressant; CM: case manager; DSM-IV: Diagnostic and Statistical Manual fourth edition; HSCL: Hopkins Symptom Checklist; MH: mental health; PCP: primary care provider

\section{Risk of bias}

\section{Bias}

Random sequence generation (selection Low risk bias)

Allocation concealment (selection bias) Unclear risk

Incomplete outcome data (attrition bias) Unclear risk All outcomes

\section{Authors' judgement}

Support for judgement

Computer generated in blocks of 8
Short-term loss to follow-up based on primary depression outcome (HSCL) was: overall 35/386 (9\%), 13/194 (7\%) intervention and 22/192 (11\%) control. Reasons for loss to follow-up not provided. 
Katon 2001 (Continued)

Used intention-to-treat analysis

\begin{tabular}{l|ll}
\hline Selective reporting (reporting bias) & Unclear risk & Insufficient information available to assess \\
\hline Other bias & Unclear risk & Insufficient information available to assess \\
\hline $\begin{array}{l}\text { Implementation Integrity } \\
\begin{array}{l}\text { Blinding of participants and personnel } \\
\text { (performance bias) } \\
\text { All outcomes }\end{array}\end{array}$ & High risk & Insufficient information available to assess \\
\hline
\end{tabular}

Blinding of outcome assessment (detection Low risk

Assessor was not aware of treatment allocabias)

tion

All outcomes

Katon 2004

\begin{tabular}{ll} 
Methods & Study design: Randomised controlled trial \\
\hline Participants & Setting: Primary care \\
& Diagnosis: Although patients were not required to meet criteria for major depression, \\
they were required to have a score of 10 or greater on the PHQ-9 in the initial screening \\
and persistent symptoms, as evidenced by a Symptom Checklist (HSCL-90) depression \\
mean item score of higher than 1.1 at a second telephone screen 2 weeks later \\
Inclusion criteria: Diabetic patients, ambulatory, English speaking, had adequate hearing \\
to complete a telephone interview, and planned to continue to be enrolled in insurance \\
plan during the next year \\
Exclusion criteria: Currently in care with a psychiatrist, bipolar disorder or schizophre- \\
nia, use of antipsychotic or mood stabiliser medication, mental confusion on interview \\
suggesting significant dementia \\
Age: Mean 58.4 years \\
Gender: $65 \%$ female \\
Ethnicity: $79 \%$ white \\
Country: United States \\
Sample size (randomised): Total participants 329, intervention 164, control 165
\end{tabular}

Interventions

Intervention: Stepped collaborative care

Contains the four elements of collaborative care:

1) a multi-professional approach to patient care: Primary care provider (PCP), nurse (CM), psychologist/psychiatrist (MH specialist)

2) a structured management plan: Step 1: Initial choice of ADs or problem solving therapy (PST). Step 2: If poor response after 10 to 12 weeks they could (1) switch to a different $\mathrm{AD}$; (2) switch to the alternative treatment (from PST to medication or vice versa); (3) receive augmentation with $\mathrm{PST}$ or $\mathrm{AD}$; or (4) receive a psychiatric consultation. Step 3: For continued non-response a referral was made to specialty care. Once patients reached a significant decrease in clinical symptoms CMs began continuation phase treatment, which consisted of monthly scheduled telephone contacts 
3) scheduled patient follow-ups: Acute phase: Assessment plus twice-a-month telephone and in-person appointments. Continuation phase: monthly telephone calls or monthly continuation groups

4) enhanced inter-professional communication: CMs interacted regularly (via written notes and verbally) with PCP. CMs had supervision twice a month with a $\mathrm{MH}$ specialist team to review new cases and patient progress. On alternate weeks, CMs reviewed cases by telephone with the psychiatrist supervisor

Control: Treatment as usual and patients were advised to consult with their PCP regarding depression

$\begin{array}{ll}\text { Outcomes } & \text { Depression (HSCL-90): 3, 6, 12, } 24 \text { months } \\ & \text { Medication use: 3, 6, 9, 12, 24 months } \\ & \text { Satisfaction: 6, 12 months }\end{array}$

Notes

AD: antidepressant; CM: case manager; HSCL: Hopkins Symptom Checklist; MH: mental health; PCP: primary care provider; PHQ-9: Patient Health Questionnaire

\section{Risk of bias}

\begin{tabular}{|c|c|c|}
\hline Bias & Authors' judgement & Support for judgement \\
\hline $\begin{array}{l}\text { Random sequence generation (selection } \\
\text { bias) }\end{array}$ & Low risk & Computer generated \\
\hline Allocation concealment (selection bias) & Low risk & $\begin{array}{l}\text { An individual not involved in patient re- } \\
\text { cruitment conducted allocation }\end{array}$ \\
\hline $\begin{array}{l}\text { Incomplete outcome data (attrition bias) } \\
\text { All outcomes }\end{array}$ & Unclear risk & $\begin{array}{l}\text { Short-term loss to follow-up based on pri- } \\
\text { mary depression outcome (HSCL- } 90 \text { de- } \\
\text { pression } \geq 50 \% \text { decrease) was: overall } 37 / \\
329(11 \%), 21 / 164 \quad(13 \%) \text { intervention } \\
\text { and } 16 / 149(10 \%) \text { control. Reasons for loss } \\
\text { to follow-up not provided. Used intention- } \\
\text { to-treat analysis }\end{array}$ \\
\hline Selective reporting (reporting bias) & Low risk & $\begin{array}{l}\text { Protocol available and all prespecified out- } \\
\text { comes reported }\end{array}$ \\
\hline Other bias & Unclear risk & Insufficient information available to assess \\
\hline Implementation Integrity & Low risk & $\begin{array}{l}\text { Attempts were made to assess implemen- } \\
\text { tation integrity (e.g. direct observation or } \\
\text { rating of tapes) }\end{array}$ \\
\hline $\begin{array}{l}\text { Blinding of participants and personnel } \\
\text { (performance bias) } \\
\text { All outcomes }\end{array}$ & High risk & $\begin{array}{l}\text { Participants and personnel could not be } \\
\text { blinded, outcome likely to be influenced by } \\
\text { lack of blinding }\end{array}$ \\
\hline
\end{tabular}


Katon 2004 (Continued)

Blinding of outcome assessment (detection Low risk

Assessor was not aware of treatment allocabias)

tion

All outcomes

Katon 2010

Methods

Participants
Study design: Randomised controlled trial

Setting: Primary care

Diagnosis: Cut-off points of 3 or higher on the Patient Health Questionnaire 2 (PHQ2) and 10 or higher on the PHQ-9 used to identify patients who were eligible for the trial

Inclusion criteria: Patients with diagnoses of diabetes, coronary heart disease, or both coded according to the International Classification of Diseases (ICD9), or Current Procedural Terminology codes for coronary- artery interventions. These patients had one or more measures of poor disease control within the previous 12 months, including: blood pressure above $140 / 90 \mathrm{~mm} \mathrm{Hg}$ (based on two blood-pressure readings at separate visits within 12 months), a low-density lipoprotein (LDL) cholesterol level above 130 mg per decilitre (>3.4 mmol per litre), or a glycated haemoglobin level of $8.5 \%$ or higher. Patients who were ambulatory, spoke English, and planned to be enrolled in a healthmaintenance-organization (HMO) plan for 12 months

Exclusion criteria: Terminal illness, residence in a long-term care facility, severe hearing loss, planned bariatric surgery within 3 months, pregnancy or breastfeeding, ongoing psychiatric care, bipolar disorder or schizophrenia, use of an antipsychotic or moodstabiliser medication, and observed mental confusion suggesting dementia

Age: Mean 56.9 years

Gender: 52\% female

Ethnicity: 79\% White

Country: United States

Sample size (randomised): Total participants 214, intervention 106, control 108
Intervention: Collaborative care

Contains the four elements of collaborative care:

1) a multi-professional approach to patient care: Primary care provider (PCP), nurse (CM), psychologist/psychiatrist (MH specialist)

2) a structured management plan: CMs motivated and coached patients to solve problems and set goals for improved medication adherence and self-care (e.g. exercising and selfmonitoring of blood-pressure and glucose levels). Self-care materials, including a help book, a video compact disk on depression care, a booklet and other materials on chronic disease management, and self-monitoring devices (e.g., blood-pressure or blood-glucose meters) were provided. Patients worked collaboratively with CMs and PCPs to establish individualised clinical and self-care goals. CMs monitored the patient's progress with respect to management of depression, control of medical disease, and self-care activities. Treatment protocols guided adjustments of commonly used medicines in patients who did not achieve specific goals. CMs followed patients proactively to provide support for medication adherence

3) scheduled patient follow-ups: Assessment plus telephone or in-person contact once or twice a month until the patient achieved his or her treatment goals (treat-to-target) 
. After completion of recovery and a maintenance plan, patients were followed every 4 to 6 weeks by telephone calls from the CM to review adherence, lab test results and depression score

4) enhanced inter-professional communication: CM communicated recommended medication changes to PC. CMs received weekly supervision with $\mathrm{MH}$ specialist team to review new cases and patient progress

Control: Treatment as usual enhanced as patients were advised to consult with their PCP to receive care for depression, diabetes and/or CHD. With patient permission, PCPs were notified about depression and poor medical disease control. All study laboratory reports and results were entered into the electronic medical record

Depression (HSCL-20): 6, 12 months
Medication use: 12 months
Quality of Life (mental and physical health): 6, 12 months
Satisfaction: 6, 12 months

CHD: coronary heart disease; CM: case manager; HSCL: Hopkins Symptom Checklist; MH: mental health; PCP: primary care provider; PHQ: Patient Health Questionnaire

\section{Risk of bias}

\begin{tabular}{|c|c|c|}
\hline Bias & Authors' judgement & Support for judgement \\
\hline $\begin{array}{l}\text { Random sequence generation (selection } \\
\text { bias) }\end{array}$ & Unclear risk & $\begin{array}{l}\text { Permuted-blocks of } 4,6 \text { or } 8 \text { (randomly } \\
\text { selected) }\end{array}$ \\
\hline Allocation concealment (selection bias) & Low risk & Central allocation \\
\hline $\begin{array}{l}\text { Incomplete outcome data (attrition bias) } \\
\text { All outcomes }\end{array}$ & Unclear risk & $\begin{array}{l}\text { Short-term loss to follow-up based on pri- } \\
\text { mary depression outcome (HSCL-20) was: } \\
\text { overall } 21 / 214(10 \%), 9 / 106(8 \%) \text { inter- } \\
\text { vention and } 12 / 108(11 \%) \text { control. Rea- } \\
\text { sons for loss to follow-up not provided. } \\
\text { Used intention-to-treat analysis }\end{array}$ \\
\hline Selective reporting (reporting bias) & Low risk & $\begin{array}{l}\text { Protocol available and all prespecified out- } \\
\text { comes reported }\end{array}$ \\
\hline Other bias & Unclear risk & Insufficient information available to assess \\
\hline Implementation Integrity & Unclear risk & Insufficient information available to assess \\
\hline $\begin{array}{l}\text { Blinding of participants and personnel } \\
\text { (performance bias) } \\
\text { All outcomes }\end{array}$ & High risk & $\begin{array}{l}\text { Participants and personnel could not be } \\
\text { blinded, outcome likely to be influenced by } \\
\text { lack of blinding }\end{array}$ \\
\hline $\begin{array}{l}\text { Blinding of outcome assessment (detection } \\
\text { bias) } \\
\text { All outcomes }\end{array}$ & Low risk & $\begin{array}{l}\text { Assessor was not aware of treatment alloca- } \\
\text { tion }\end{array}$ \\
\hline
\end{tabular}


Katzelnick 2000

Methods Study design: Cluster-randomised controlled trial

Participants

Setting: Primary care

Diagnosis: Current major depression or major depression in partial remission assessed using a modified version of the Structured Clinical Interview for DSM-IV (SCID). Also a score of 15 or more on the Hamilton Depression Rating Scale (HAM-D)

Inclusion criteria: Aged 25 to 63 years with continuous health plan enrolment for the previous 2 years. High utilisers of health care defined as ambulatory visit counts above the 85 th percentile for both of the 2 previous years. Ambulatory office visits were defined as primary care visits, medical specialty visits, and walk-in clinic visits

Exclusion criteria: Recent treatment for alcohol or other substance abuse; past treatment for schizophrenia or bipolar disorder; life-threatening medical disorders (e.g., metastatic malignant neoplasm), active treatment for depression (defined as current specialty mental health treatment or minimal adequate trial of antidepressant medication), contraindications to taking an antidepressant, receiving treatment by a psychiatrist within the past 4 months, pregnancy, planned pregnancy within the next year, breastfeeding, positive screen for alcohol abuse, and intent to disenroll from the Health Maintenance Organisation

Age: Mean 45.5 years

Gender: $78 \%$ female

Ethnicity: $83 \%$ white

Country: United States

Sample size (randomised): Total clusters 163, intervention 82, control 81; Total participants 407, intervention 218, control 189

Interventions

Intervention: Depression management

Contains the four elements of collaborative care:

1) a multi-professional approach to patient care: Primary care physician (PCP), treatment co-ordinator (CM), psychiatrist (MH specialist)

2) a structured management plan: Patient education using RHYTHMs which included detail on nature of depression, use of ADs and adherence and a booklet and videotape. Specific ADs were used and CMs reviewed patient prescription refills and office visits to identify unplanned treatment discontinuation and monitored treatment adherence, treatment response and medication adverse effects

3) scheduled patient follow-ups: Telephone calls at 2 and 10 weeks and if necessary at $18,30,42$ weeks. Scheduled visits with PCP at weeks 1, 3, 6 and 10 weeks then every 10 weeks

4) enhanced inter-professional communication: CMs provided a written response to PCPs or a call if progress was not as expected or patient discontinued treatment. PCPs had periodic case reviews and as needed telephone consultation with the $\mathrm{MH}$ specialist Control: Treatment as usual

Outcomes

Depression (HAMD): 6 weeks, 3, 6, 12 months

Medication use: 6 months

Quality of Life (mental and physical health): 12 months

Notes

AD: antidepressant; CM: case manager; DSM-IV: Diagnostic and Statistical Manual fourth edition; HAM-D: Hamilton Depression Rating Scale; MH: mental health; PCP:

Collaborative care for depression and anxiety problems (Review)

Copyright $\odot 2012$ The Cochrane Collaboration. Published by John Wiley \& Sons, Ltd. 
Katzelnick 2000 (Continued)

primary care provider

\section{Risk of bias}

\begin{tabular}{|c|c|c|}
\hline Bias & Authors' judgement & Support for judgement \\
\hline $\begin{array}{l}\text { Random sequence generation (selection } \\
\text { bias) }\end{array}$ & Low risk & Computer generated \\
\hline Allocation concealment (selection bias) & Low risk & Sealed envelopes \\
\hline $\begin{array}{l}\text { Incomplete outcome data (attrition bias) } \\
\text { All outcomes }\end{array}$ & Unclear risk & $\begin{array}{l}\text { Short-term loss to follow-up based on pri- } \\
\text { mary depression outcome (HAMD) was: } \\
\text { overall } 26 / 407(6 \%), 16 / 218(7 \%) \text { inter- } \\
\text { vention and } 10 / 189(5 \%) \text { control. Reasons } \\
\text { for loss to follow-up not provided. Used in- } \\
\text { tention-to-treat analysis }\end{array}$ \\
\hline Selective reporting (reporting bias) & Unclear risk & Insufficient information available to assess \\
\hline Other bias & Unclear risk & Insufficient information available to assess \\
\hline Implementation Integrity & Unclear risk & Insufficient information available to assess \\
\hline $\begin{array}{l}\text { Blinding of participants and personnel } \\
\text { (performance bias) } \\
\text { All outcomes }\end{array}$ & High risk & $\begin{array}{l}\text { Participants and personnel could not be } \\
\text { blinded, outcome likely to be influenced by } \\
\text { lack of blinding }\end{array}$ \\
\hline $\begin{array}{l}\text { Blinding of outcome assessment (detection } \\
\text { bias) } \\
\text { All outcomes }\end{array}$ & Low risk & $\begin{array}{l}\text { Assessor was not aware of treatment alloca- } \\
\text { tion }\end{array}$ \\
\hline
\end{tabular}

Kroenke 2010

Methods

Participants
Study design: Randomised controlled trial

Setting: Specialist

Diagnosis: At least moderately severe depression, defined as a PHQ-9 score of 10 or higher and endorsement of either depressed mood, anhedonia; or both

Inclusion criteria: Patients presenting for oncology clinic visits who screened positive for either pain or depression. Pain had to be (1) definitely or possibly cancer related; (2) at least moderately severe, (3) persistent despite trying at least 1 pain medicine

Exclusion criteria: Unable to speak English, moderately severe cognitive impairment, schizophrenia or other psychosis, had a pending pain related disability claim, were pregnant, or were in hospice care

Age: Mean 58.9 years

Gender: $68 \%$ female

Ethnicity: $80 \%$ White 
Kroenke 2010 (Continued)

Country: United States

Sample size (randomised): Total participants 405, intervention 202, control 203

Interventions

Intervention: Care management

Contains the four elements of collaborative care:

1) a multi-professional approach to patient care: Oncologist (PCP), nurse (CM), painpsychiatrist (MH specialist)

2) a structured management plan: CMs assessed symptom response and medication adherence; provided pain and depression specific education; and made treatment adjustments according to evidence based guidelines. Automated symptom monitoring was also performed between sessions using interactive voice recorded telephone calls or web based surveys. Participants who preferred not to take ADs were encouraged to consider a referral for psychotherapy and speak to their oncologist re this

3) scheduled patient follow-ups: 4 in 12 weeks: at baseline, 1, 4 and 12 weeks plus automated contact

4) enhanced inter-professional communication: Treatment recommendations were provided to PCP. CM had weekly supervision with $\mathrm{MH}$ specialist who was available between sessions

Control: Treatment as usual enhanced as patients were informed of their depressive and pain symptoms, and their screening results were provided to PCP

Outcomes

Depression (HSCL-20): 1, 3, 6, 12 months

Quality of Life (mental and physical health): 1, 3, 6, 12 months

Notes

AD: antidepressant; CM: case manager; HSCL: Hopkins Symptom Checklist; MH: mental health; PCP: primary care provider; PHQ-9: Patient Health Questionnaire-9

Risk of bias

\begin{tabular}{|c|c|c|}
\hline Bias & Authors' judgement & Support for judgement \\
\hline $\begin{array}{l}\text { Random sequence generation (selection } \\
\text { bias) }\end{array}$ & Low risk & $\begin{array}{l}\text { Computer generated in blocks of } 4,8 \text { and } \\
12 \text { (randomly selected) }\end{array}$ \\
\hline Allocation concealment (selection bias) & Unclear risk & Insufficient information available to assess \\
\hline $\begin{array}{l}\text { Incomplete outcome data (attrition bias) } \\
\text { All outcomes }\end{array}$ & Unclear risk & $\begin{array}{l}\text { Short-term loss to follow-up based on pri- } \\
\text { mary depression outcome (HSCL-20) was: } \\
\text { overall } 182 / 405(45 \%), 92 / 202(46 \%) \text { in- } \\
\text { tervention and } 90 / 203(44 \%) \text { control. Rea- } \\
\text { sons for loss to follow-up provided, with } \\
\text { similar reasons for missing data across } \\
\text { groups. Intention-to-treat analysis reported } \\
\text { with appropriate imputation methods to } \\
\text { manage missing data }\end{array}$ \\
\hline
\end{tabular}

Selective reporting (reporting bias) Low risk

Protocol available and all prespecified outcomes reported 
Kroenke 2010 (Continued)

\begin{tabular}{|c|c|c|}
\hline Other bias & Unclear risk & Insufficient information available to assess \\
\hline Implementation Integrity & Unclear risk & Insufficient information available to assess \\
\hline $\begin{array}{l}\text { Blinding of participants and personnel } \\
\text { (performance bias) } \\
\text { All outcomes }\end{array}$ & High risk & $\begin{array}{l}\text { Participants and personnel could not be } \\
\text { blinded, outcome likely to be influenced by } \\
\text { lack of blinding }\end{array}$ \\
\hline $\begin{array}{l}\text { Blinding of outcome assessment (detection } \\
\text { bias) } \\
\text { All outcomes }\end{array}$ & Low risk & $\begin{array}{l}\text { Assessor was not aware of treatment alloca- } \\
\text { tion }\end{array}$ \\
\hline
\end{tabular}

Landis 2007

Methods

Participants
Study design: Randomised controlled trial

Setting: Primary care

Diagnosis: Significant depression determined by a score of 10 or more on the PHQ-9. The primary care physician verified the presence of major depression by clinical exam Inclusion criteria: Willing to begin or continue antidepressant medication Exclusion criteria: Bipolar disorder, psychotic symptoms, active suicidal ideation requiring psychiatric admission

Age: Mean 39.7 (SD 10.7) years

Gender: 96\% female

Ethnicity: $62 \%$ white

Country: United States

Sample size (randomised): Total participants 45, intervention 22, control 23
Intervention: Care management

Contains the four elements of collaborative care:

1) a multi-professional approach to patient care: Primary care physician (PCP), mental health graduate $(\mathrm{CM})$, psychiatrist ( $\mathrm{MH}$ specialist)

2) a structured management plan: CMs provided patient education about depression and instruction in self-management skills and goals and monitored adherence and side effects

3) scheduled patient follow-ups: Acute: telephone or in-person every 2 weeks for up-to 12 weeks, maintenance: every 4 weeks until 6 months post-initial session

4) enhanced inter-professional communication: CMs coordinated with the PCP and received bi-weekly telephone supervision from $\mathrm{MH}$ specialist

Control: Treatment as usual enhanced as PCP was informed of diagnosis

Outcomes

Depression (PHQ-9): 3, 6 months

Medication use: 3, 6 months

Quality of Life (mental and physical health): 3, 6 months

Satisfaction: 3, 6 months

Notes

CM: case manager; $\mathrm{MH}$ : mental health; PCP: primary care provider; PHQ-9: Patient Health Questionnaire-9 
Landis 2007 (Continued)

\section{Risk of bias}

\begin{tabular}{|c|c|c|}
\hline Bias & Authors' judgement & Support for judgement \\
\hline $\begin{array}{l}\text { Random sequence generation (selection } \\
\text { bias) }\end{array}$ & Unclear risk & $\begin{array}{l}\text { Random assignment of } 200 \text { study numbers } \\
\text { pre-trial. Stratified by newly diagnosed and } \\
\text { already treated with AD medication }\end{array}$ \\
\hline Allocation concealment (selection bias) & Low risk & Sealed envelope \\
\hline $\begin{array}{l}\text { Incomplete outcome data (attrition bias) } \\
\text { All outcomes }\end{array}$ & High risk & $\begin{array}{l}\text { Short-term loss to follow-up based on pri- } \\
\text { mary depression outcome (PHQ-9) was: } \\
\text { overall } 11 / 45(24 \%), 5 / 22(23 \%) \text { interven- } \\
\text { tion and } 6 / 23(26 \%) \text { control. Reasons for } \\
\text { loss to follow-up not provided. Intention- } \\
\text { to-treat analysis not reported, no descrip- } \\
\text { tion of methods to manage missing data }\end{array}$ \\
\hline Selective reporting (reporting bias) & Unclear risk & Insufficient information available to assess \\
\hline Other bias & Unclear risk & Insufficient information available to assess \\
\hline Implementation Integrity & Unclear risk & Insufficient information available to assess \\
\hline $\begin{array}{l}\text { Blinding of participants and personnel } \\
\text { (performance bias) } \\
\text { All outcomes }\end{array}$ & High risk & $\begin{array}{l}\text { Participants and personnel could not be } \\
\text { blinded, outcome likely to be influenced by } \\
\text { lack of blinding }\end{array}$ \\
\hline $\begin{array}{l}\text { Blinding of outcome assessment (detection } \\
\text { bias) } \\
\text { All outcomes }\end{array}$ & Low risk & $\begin{array}{l}\text { Assessor was not aware of treatment alloca- } \\
\text { tion }\end{array}$ \\
\hline
\end{tabular}

Lobello 2010

Methods

Participants
Study design: Randomised controlled trial

Setting: Primary care

Diagnosis: Primary diagnosis of major depressive disorder assessed using a modified MiniInternational Neuropsychiatric Interview (MINI), and a diagnosis of major depressive disorder, single or recurrent episode without psychotic features, was confirmed according to Diagnostic and Statistical Manual of Mental Disorders(DSM-IV) criteria. Patients were required to have a minimum Hamilton Rating Scale for Depression (HAM-D17) score of 14

Inclusion criteria: Male and female outpatients aged 18 years or older. Sexually active women of child bearing potential were required to use medically acceptable contraception Exclusion criteria: Current treatment with venlafaxine or previously failed venlafaxine treatment at adequate dose and duration; significant risk of suicide based on clinical 
Lobello 2010 (Continued)

judgment; pregnancy or breastfeeding; introduction or change in cognitive behavioural therapy, interpersonal therapy, or other psychotherapy within 3 months before randomisation; and concomitant use of other psychopharmacologic drugs

Age: Mean 44.5 years

Gender: 73\% female

Ethnicity: $87 \%$ white

Country: United States

Sample size (randomised): Total participants 537, intervention 268, control 269

Interventions

Intervention: Venlafaxine ER plus Dialogues programme

Contains the four elements of collaborative care:

1) a multi-professional approach to patient care: Primary care physician (PCP), nurse (CM)

2) a structured management plan: The Dialogues programme included a welcome kit that included the first issue of the Dialogues Magazine, a Straight Talk booklet (on side effects), and a tip sheet (points to discuss with PCP). Over a 4-month period, patients also received a comprehensive resource guide, 2 additional issues of the Dialogues Magazine, and 3 additional Straight Talk booklets (progress, managing stress, long-term therapy) 3) scheduled patient follow-ups: 3 planned periodic calls (weeks 1, 5 and 13) and access to a 12-hour daily help line

4) enhanced inter-professional communication: After each telephone call a contact report was sent to the PCP

Control: The venlafaxine ER group received venlafaxine ER as part of the standard practice of care for the treatment of major depression

Outcomes

Depression (HAM-D): 14, 45, 112, 135, 180 days

Medication use: $14,45,112,135,180$ days

Quality of Life (mental and physical health): 14, 45, 112, 135, 180 days

Satisfaction: $14,45,112,135,180$ days

Notes

CM: case manager; HAM-D: Hamilton Depression Rating Scale; MH: mental health; PCP: primary care provider

Risk of bias

\begin{tabular}{|c|c|c|}
\hline Bias & Authors' judgement & Support for judgement \\
\hline $\begin{array}{l}\text { Random sequence generation (selection } \\
\text { bias) }\end{array}$ & Unclear risk & Insufficient information available to assess \\
\hline Allocation concealment (selection bias) & Unclear risk & Insufficient information available to assess \\
\hline $\begin{array}{l}\text { Incomplete outcome data (attrition bias) } \\
\text { All outcomes }\end{array}$ & Low risk & $\begin{array}{l}\text { Short-term loss to follow-up based on pri- } \\
\text { mary depression outcome (HAMD remis- } \\
\text { sion total score } \leq 7) \text { was: overall } 45 / \\
537(8 \%), 29 / 268(11 \%) \text { intervention and } \\
16 / 269(6 \%) \text { control. Reasons for loss to } \\
\text { follow-up provided, with similar reasons } \\
\text { across groups. Used intention-to-treat anal- }\end{array}$ \\
\hline
\end{tabular}

Collaborative care for depression and anxiety problems (Review) 
Lobello 2010 (Continued)

ysis

\begin{tabular}{l|l|l}
\hline Selective reporting (reporting bias) & Unclear risk & Insufficient information available to assess \\
\hline Other bias & Unclear risk & Insufficient information available to assess \\
\hline Implementation Integrity & Unclear risk & Insufficient information available to assess \\
\hline $\begin{array}{l}\text { Blinding of participants and personnel } \\
\text { (performance bias) } \\
\text { All outcomes }\end{array}$ & High risk & $\begin{array}{l}\text { Participants and personnel could not be } \\
\text { blinded, outcome likely to be influenced by } \\
\text { lack of blinding }\end{array}$ \\
\hline
\end{tabular}

Blinding of outcome assessment (detection Unclear risk

Insufficient information available to assess bias)

All outcomes

Ludman 2007a

\begin{tabular}{|c|c|}
\hline Methods & Study design: Pilot randomised controlled trial \\
\hline Participants & $\begin{array}{l}\text { Setting: Community, primary care } \\
\text { Diagnosis: Persistent symptoms of depression despite at least six months of antidepressant } \\
\text { treatment prescribed in specialty care. Assessed by a score of } 0.75 \text { or above on a } 20 \text { - } \\
\text { item depression scale extracted from the } 90 \text {-item Hopkins Symptom Checklist (HSCL- } \\
\text { 90). At least one major depressive episode in the past two years as diagnosed by a } \\
\text { structured interview and a history of either recurrent major depression (more than three } \\
\text { episodes in the past five years) or dysthymia. All patients met criteria for recurrent } \\
\text { major depression or dysthymia, but were heterogeneous with respect to current mood } \\
\text { state (dysthymia, chronic major depression, partial remission, relapse, or recurrence) and } \\
\text { current antidepressant treatment } \\
\text { Inclusion criteria: Aged } 18 \text { and older who had initiated antidepressant treatment at least } \\
180 \text { days previously, had a visit diagnosis of major depressive disorder at the time of } \\
\text { initial antidepressant prescription, and were continuously enrolled in insurance group } \\
\text { for at least the previous } 180 \text { days } \\
\text { Exclusion criteria: History of mania or hypomania, cognitive impairment, near-terminal } \\
\text { medical illness, intent to disenroll from insurance group within the next } 12 \text { months, } \\
\text { emergent clinical needs (for example, risk of harm to self or others), diagnosis of bipolar } \\
\text { disorder or psychotic disorder or prescription for a mood stabiliser or antipsychotic } \\
\text { medication in the past two years } \\
\text { Age: Mean } 50.3 \text { years } \\
\text { Gender: } 69 \% \text { female } \\
\text { Ethnicity: } 87 \% \text { caucasian } \\
\text { Country: United States } \\
\text { Sample size (randomised): Total participants } 52 \text {, intervention } 26 \text {, control } 26\end{array}$ \\
\hline
\end{tabular}

Interventions

Intervention: Telephone care management.

Contains the four elements of collaborative care:

1) a multi-professional approach to patient care: Primary care provider (PCP), counsellor 
Ludman 2007a (Continued)

(CM), psychologist/psychiatrist (MH specialist)

2) a structured management plan: Using computer-assisted scripts, CMs provided education about medication adherence and management of side effects and incorporated motivational enhancement strategies. CM also provided any needed outreach and care coordination, including facilitation of follow-up care

3) scheduled patient follow-ups: $\mathrm{CM}=3$ monthly telephone calls plus others if required. Peer group $=6$ weekly sessions plus additional bimonthly group. Psychotherapy group= 10 weekly sessions plus 3 booster sessions

4) enhanced inter-professional communication: After each contact, CMs sent the PCP a report of current symptoms, medication use, side effects, prior treatment, and algorithmbased recommendations. In the case of moderate or severe symptoms CMs communicated with the PCP by telephone within 24 hours

Control: Treatment as usual

Outcomes

Depression (HSCL): 3, 6, 9, 12 months

Medication use: 12 months

Satisfaction: 6, 9, 12 months

Notes

CM: case manager; HSCL: Hopkins Symptom Checklist; MH: mental health; PCP: primary care provider

\section{Risk of bias}

\begin{tabular}{|c|c|c|}
\hline Bias & Authors' judgement & Support for judgement \\
\hline $\begin{array}{l}\text { Random sequence generation (selection } \\
\text { bias) }\end{array}$ & Low risk & Computer generated block randomisation \\
\hline
\end{tabular}

\begin{tabular}{|c|c|c|}
\hline Allocation concealment (selection bias) & Low risk & $\begin{array}{l}\text { Allocation conducted by an individual not } \\
\text { involved in patient recruitment }\end{array}$ \\
\hline $\begin{array}{l}\text { Incomplete outcome data (attrition bias) } \\
\text { All outcomes }\end{array}$ & Unclear risk & $\begin{array}{l}\text { Short-term loss to follow-up based on pri- } \\
\text { mary depression outcome (HSCL depres- } \\
\text { sion) was: overall } 7 / 52(13 \%), 5 / 26(19 \%) \\
\text { intervention and } 2 / 26(8 \%) \text { control. Rea- } \\
\text { sons for loss to follow-up not provided. } \\
\text { Used intention-to-treat analysis, no de- } \\
\text { scription of methods to manage missing } \\
\text { data }\end{array}$ \\
\hline Selective reporting (reporting bias) & Unclear risk & Insufficient information available to assess \\
\hline Other bias & Unclear risk & Insufficient information available to assess \\
\hline Implementation Integrity & Low risk & $\begin{array}{l}\text { Attempts were made to assess implemen- } \\
\text { tation integrity (e.g. direct observation or } \\
\text { rating of tapes) }\end{array}$ \\
\hline
\end{tabular}


Ludman 2007a (Continued)

Blinding of participants and personnel High risk (performance bias)

All outcomes
Participants and personnel could not be blinded, outcome likely to be influenced by lack of blinding

Assessor was not aware of treatment allocation

bias)

All outcomes

Ludman 2007b

\section{Methods}

Participants
Study design: Pilot randomised controlled trial

Setting: Community, primary care

Diagnosis: Persistent symptoms of depression despite at least six months of antidepressant treatment prescribed in specialty care. Assessed by a score of 0.75 or above on a 20item depression scale extracted from the 90-item Hopkins Symptom Checklist (HSCL90). At least one major depressive episode in the past two years as diagnosed by a structured interview and a history of either recurrent major depression (more than three episodes in the past five years) or dysthymia. All patients met criteria for recurrent major depression or dysthymia, but were heterogeneous with respect to current mood state (dysthymia, chronic major depression, partial remission, relapse, or recurrence) and current antidepressant treatment

Inclusion criteria: Aged 18 and older who had initiated antidepressant treatment at least 180 days previously, had a visit diagnosis of major depressive disorder at the time of initial antidepressant prescription, and were continuously enrolled in insurance group for at least the previous 180 days

Exclusion criteria: History of mania or hypomania, cognitive impairment, near-terminal medical illness, intent to disenroll from insurance group within the next 12 months, emergent clinical needs (for example, risk of harm to self or others), diagnosis of bipolar disorder or psychotic disorder or prescription for a mood stabiliser or antipsychotic medication in the past two years

Age: Mean 50.7 years

Gender: $69 \%$ female

Ethnicity: $85 \%$ caucasian

Country: United States

Sample size (randomised): Total participants 52, intervention 26, control 26

Interventions

Intervention: Telephone care management plus a peer-led chronic-disease self-management group programme

Contains the four elements of collaborative care:

1) a multi-professional approach to patient care: Primary care provider (PCP), counsellor (CM), psychologist/psychiatrist (MH specialist)

2) a structured management plan: Using computer-assisted scripts, CMs provided education about medication adherence and management of side effects and incorporated motivational enhancement strategies. CM also provided any needed outreach and care coordination, including facilitation of follow-up care

$3)$ scheduled patient follow-ups: $\mathrm{CM}=3$ monthly telephone calls plus others if required.

Peer group $=6$ weekly sessions plus additional bimonthly group. Psychotherapy group= 
Ludman 2007b (Continued)

10 weekly sessions plus 3 booster sessions

4) enhanced inter-professional communication: After each contact, CMs sent the PCP a report of current symptoms, medication use, side effects, prior treatment, and algorithmbased recommendations. In the case of moderate or severe symptoms CMs communicated with the PCP by telephone within 24 hours

Control: Treatment as usual

Outcomes

Depression (HSCL): 3, 6, 9, 12 months

Medication use: 12 months

Satisfaction: 6, 9, 12 months

Notes

CM: case manager; HSCL: Hopkins Symptom Checklist; MH: mental health; PCP: primary care provider

\section{Risk of bias}

\begin{tabular}{|c|c|c|}
\hline Bias & Authors' judgement & Support for judgement \\
\hline $\begin{array}{l}\text { Random sequence generation (selection } \\
\text { bias) }\end{array}$ & Low risk & Computer generated block randomisation \\
\hline Allocation concealment (selection bias) & Low risk & $\begin{array}{l}\text { Allocation conducted by an individual not } \\
\text { involved in patient recruitment }\end{array}$ \\
\hline $\begin{array}{l}\text { Incomplete outcome data (attrition bias) } \\
\text { All outcomes }\end{array}$ & Unclear risk & $\begin{array}{l}\text { Short-term loss to follow-up based on pri- } \\
\text { mary depression outcome (HSCL depres- } \\
\text { sion) was: overall } 4 / 52(8 \%), 2 / 26(8 \%) \text { in- } \\
\text { tervention and } 2 / 26(8 \%) \text { control. Reasons } \\
\text { for loss to follow-up not provided. Used in- } \\
\text { tention-to-treat analysis }\end{array}$ \\
\hline Selective reporting (reporting bias) & Unclear risk & Insufficient information available to assess \\
\hline Other bias & Unclear risk & Insufficient information available to assess \\
\hline Implementation Integrity & Low risk & $\begin{array}{l}\text { Attempts were made to assess implemen- } \\
\text { tation integrity (e.g. direct observation or } \\
\text { rating of tapes) }\end{array}$ \\
\hline $\begin{array}{l}\text { Blinding of participants and personnel } \\
\text { (performance bias) } \\
\text { All outcomes }\end{array}$ & High risk & $\begin{array}{l}\text { Participants and personnel could not be } \\
\text { blinded, outcome likely to be influenced by } \\
\text { lack of blinding }\end{array}$ \\
\hline $\begin{array}{l}\text { Blinding of outcome assessment (detection } \\
\text { bias) } \\
\text { All outcomes }\end{array}$ & Low risk & $\begin{array}{l}\text { Assessor was not aware of treatment alloca- } \\
\text { tion }\end{array}$ \\
\hline
\end{tabular}



treatment prescribed in specialty care. Assessed by a score of 0.75 or above on a 20item depression scale extracted from the 90-item Hopkins Symptom Checklist (HSCL90). At least one major depressive episode in the past two years as diagnosed by a structured interview and a history of either recurrent major depression (more than three episodes in the past five years) or dysthymia. All patients met criteria for recurrent major depression or dysthymia, but were heterogeneous with respect to current mood state (dysthymia, chronic major depression, partial remission, relapse, or recurrence) and current antidepressant treatment

Inclusion criteria: Aged 18 and older who had initiated antidepressant treatment at least 180 days previously, had a visit diagnosis of major depressive disorder at the time of initial antidepressant prescription, and were continuously enrolled in insurance plan for at least the previous 180 days

Exclusion criteria: History of mania or hypomania, cognitive impairment, near-terminal medical illness, intent to disenroll from insurance plan within the next 12 months, emergent clinical needs (for example, risk of harm to self or others), diagnosis of bipolar disorder or psychotic disorder or prescription for a mood stabiliser or antipsychotic medication in the past two years

Age: Mean 50.5 years

Gender: $73 \%$ female

Ethnicity: $81 \%$ caucasian

Country: United States

Sample size (randomised): Total participants 52, intervention 26, control 26

Intervention: Telephone care management plus a professionally led depression psychotherapy group

Contains the four elements of collaborative care:

1) a multi-professional approach to patient care: Primary care provider (PCP), counsellor (CM), psychologist/psychiatrist (MH specialist)

2) a structured management plan: Using computer-assisted scripts, CMs provided education about medication adherence and management of side effects and incorporated motivational enhancement strategies. CM also provided any needed outreach and care coordination, including facilitation of follow-up care

3) scheduled patient follow-ups: $\mathrm{CM}=3$ monthly telephone calls plus others if required. Peer group $=6$ weekly sessions plus additional bimonthly group. Psychotherapy group = 10 weekly sessions plus 3 booster sessions

4) enhanced inter-professional communication: After each contact, CMs sent the PCP a report of current symptoms, medication use, side effects, prior treatment, and algorithmbased recommendations. In the case of moderate or severe symptoms CMs communicated with the PCP by telephone within 24 hours

Control: Treatment as usual 
Ludman 2007c (Continued) $\begin{array}{ll}\text { Notes } & \begin{array}{l}\text { CM: case manager; HSCL: Hopkins Symptom Checklist; MH: mental health; PCP: } \\ \text { primary care provider }\end{array}\end{array}$

\section{Risk of bias}

\begin{tabular}{|c|c|c|}
\hline Bias & Authors' judgement & Support for judgement \\
\hline $\begin{array}{l}\text { Random sequence generation (selection } \\
\text { bias) }\end{array}$ & Low risk & Computer generated block randomisation \\
\hline Allocation concealment (selection bias) & Low risk & $\begin{array}{l}\text { Allocation conducted by an individual not } \\
\text { involved in patient recruitment }\end{array}$ \\
\hline $\begin{array}{l}\text { Incomplete outcome data (attrition bias) } \\
\text { All outcomes }\end{array}$ & Unclear risk & $\begin{array}{l}\text { Short-term loss to follow-up based on pri- } \\
\text { mary depression outcome (HSCL depres- } \\
\text { sion) was: overall } 6 / 52(12 \%), 4 / 26(15 \%) \\
\text { intervention and } 2 / 26(8 \%) \text { control. Rea- } \\
\text { sons for loss to follow-up not provided. } \\
\text { Used intention-to-treat analysis }\end{array}$ \\
\hline Selective reporting (reporting bias) & Unclear risk & Insufficient information available to assess \\
\hline Other bias & Unclear risk & Insufficient information available to assess \\
\hline Implementation Integrity & Low risk & $\begin{array}{l}\text { Attempts were made to assess implemen- } \\
\text { tation integrity (e.g. direct observation or } \\
\text { rating of tapes) }\end{array}$ \\
\hline $\begin{array}{l}\text { Blinding of participants and personnel } \\
\text { (performance bias) } \\
\text { All outcomes }\end{array}$ & High risk & $\begin{array}{l}\text { Participants and personnel could not be } \\
\text { blinded, outcome likely to be influenced by } \\
\text { lack of blinding }\end{array}$ \\
\hline $\begin{array}{l}\text { Blinding of outcome assessment (detection } \\
\text { bias) } \\
\text { All outcomes }\end{array}$ & Low risk & $\begin{array}{l}\text { Assessor was not aware of treatment alloca- } \\
\text { tion }\end{array}$ \\
\hline
\end{tabular}

\section{Mann 1998}

Methods

Participants
Study design: Randomised controlled trial

Setting: Primary care

Diagnosis: General Practitioners assessed as depressed. Severity defined after referral by use of the Beck Depression Inventory (BDI) and Nurse Assessment Interview

Inclusion criteria: Patients aged 18-74 years, who had been depressed for at least four weeks. Those currently receiving treatment from their GP for depression or presenting with a new episode were included

Exclusion criteria: Suicidal ideation, those whose depression represented a phase in a manic-depressive psychosis, and those currently receiving treatment for depression from 
specialist psychiatric services

Age: Mean 45.7 years

Gender: $78 \%$ female

Ethnicity: Not stated

Country: United Kingdom

Sample size (randomised): Total participants 419, intervention 271, control 148

Interventions

Intervention: Nurse assisted follow-up care

Contains the four elements of collaborative care:

1) a multi-professional approach to patient care: General Practitioner (PCP), nurse (CM)

2) a structured management plan: CMs assessed patients using a depression measurement tool and reported result to PCP. CMs worked to a manual that covered: strategies to improve compliance (the CM explained the rationale of treatment by medication, helped manage side-effects, and discussed dose changes with PCP), education of patients (leaflets on depression were included in the manual for CMs to explore and explain depression and, if necessary, provide to patients), initiation of social interventions (CMs made contact with local support agencies that might help depressed patients) and CMs also made contact with the local specialist psychiatric services

3) scheduled patient follow-ups: Regularly during first month with no specific regimen thereafter

4) enhanced inter-professional communication: The CM discussed each patient with the PCP, who decided upon treatment. CMs could discuss a patient with the PCP at any time. Nurses received supervision from other nurses who had done similar work previously

Control: Treatment as usual

\section{Risk of bias}

\begin{tabular}{|c|c|c|}
\hline Bias & Authors' judgement & Support for judgement \\
\hline $\begin{array}{l}\text { Random sequence generation (selection } \\
\text { bias) }\end{array}$ & Low risk & Random number table \\
\hline Allocation concealment (selection bias) & Low risk & Sealed envelope \\
\hline $\begin{array}{l}\text { Incomplete outcome data (attrition bias) } \\
\text { All outcomes }\end{array}$ & Unclear risk & $\begin{array}{l}\text { Short-term loss to follow-up based on pri- } \\
\text { mary depression outcome (DSM-III de- } \\
\text { pression) was: overall } 34 / 419(8 \%), 20 / 271 \\
(7 \%) \text { intervention and } 14 / 148(9 \%) \text { con- } \\
\text { trol. Reasons for loss to follow-up not pro- } \\
\text { vided. Used intention-to-treat analysis }\end{array}$ \\
\hline Selective reporting (reporting bias) & Unclear risk & Insufficient information available to assess \\
\hline
\end{tabular}


Mann 1998 (Continued)

\begin{tabular}{l|l|l}
\hline Other bias & Unclear risk & Insufficient information available to assess \\
\hline Implementation Integrity & Unclear risk & Insufficient information available to assess \\
\hline $\begin{array}{l}\text { Blinding of participants and personnel } \\
\text { (performance bias) } \\
\begin{array}{l}\text { All outcomes } \\
\text { Blinding of outcome assessment (detection }\end{array}\end{array}$ & Unclear risk \\
$\begin{array}{l}\text { bias) } \\
\text { All outcomes }\end{array}$ & & $\begin{array}{l}\text { Participants and personnel could not be } \\
\text { blinded, outcome likely to be influenced by } \\
\text { lack of blinding }\end{array}$ \\
\hline
\end{tabular}

McCusker 2008

Methods

Participants
Study design: Pilot cluster-randomised controlled trial

Setting: Primary care

Diagnosis: A positive response to either of the PHQ-2 screening questions, followed by a screen for major depression using a Structured Clinical Interview (SCID)

Inclusion criteria: Able to speak English or French well enough to be able to complete study questionnaires, aged 60 or over, reside in an area easily accessible for the depression care worker, with a maximum travel time of 45 minutes in each direction, see the participating family physician as their principal primary care physician

Exclusion criteria: In active treatment with a Psychiatrist or Psychologist, bipolar, psychosis, hearing impairment, and ability to provide consent (no or only minimal cognitive impairment)

Age: Mean 73.3 years

Gender: 67\% female

Ethnicity: Not stated

Country: Canada

Sample size (randomised): Total participants 68, intervention 36, control 32

Interventions

Intervention: Depression Care Practitioner

Contains the four elements of collaborative care:

1) a multi-professional approach to patient care: Family physician (PCP), social worker/ psychologist $(\mathrm{CM})$, psychiatrist (MH specialist)

2) a structured management plan: Patients were provided with an educational brochure and a video prepared for the IMPACT study. CMs assessed and worked with each patient and PCP to develop a treatment plan that could include pharmacotherapy using an evidence-based medication algorithm and 4 sessions of problem solving therapy (PST). During follow-up CMs monitored the patient's progress

3) scheduled patient follow-ups: Assessment plus 4 weekly sessions (telephone or faceto-face)

4) enhanced inter-professional communication: CMs sent the PCP a copy of the assessment and all details of follow-up sessions. CMs had weekly supervision with $\mathrm{MH}$ specialist. The $\mathrm{MH}$ specialist reviewed all medication recommendations proposed by PCP, was available for rapid verbal consultations with physicians and CMs, and offered an immediate consultation when needed 
McCusker 2008 (Continued)

Control: Treatment as usual enhanced as PCPs were informed of patients diagnosis

\begin{tabular}{ll}
\hline Outcomes & $\begin{array}{l}\text { Depression (HSCL): } 2 \text { months } \\
\text { Medication use: } 2 \text { months } \\
\text { Satisfaction: } 2 \text { months }\end{array}$ \\
\hline Notes & $\begin{array}{l}\text { CM: case manager; HSCL: Hopkins Symptom Checklist; MH: mental health; PCP: } \\
\text { primary care provider }\end{array}$ \\
\hline
\end{tabular}

\section{Risk of bias}

\begin{tabular}{|c|c|c|}
\hline Bias & Authors' judgement & Support for judgement \\
\hline $\begin{array}{l}\text { Random sequence generation (selection } \\
\text { bias) }\end{array}$ & Unclear risk & $\begin{array}{l}\text { Physicians were randomised based on their } \\
\text { preference and size and type of practice, in } \\
\text { the patient randomised arm a prepared list } \\
\text { of random numbers in blocks of } 4 \text { were } \\
\text { used }\end{array}$ \\
\hline Allocation concealment (selection bias) & Unclear risk & $\begin{array}{l}\text { Standard block size. Allocation of patients } \\
\text { conducted by study coordinator }\end{array}$ \\
\hline $\begin{array}{l}\text { Incomplete outcome data (attrition bias) } \\
\text { All outcomes }\end{array}$ & Unclear risk & $\begin{array}{l}\text { Short-term loss to follow-up based on pri- } \\
\text { mary depression outcome (HSCL) was: } \\
\text { overall } 2 / 34(6 \%), 0 / 19(0 \%) \text { intervention } \\
\text { and } 2 / 15(13 \%) \text { control. Reasons for loss } \\
\text { to follow-up provided and only observed } \\
\text { in control group. Intention-to-treat analy- } \\
\text { sis not reported, no description of methods } \\
\text { to manage missing data }\end{array}$ \\
\hline Selective reporting (reporting bias) & Unclear risk & Insufficient information available to assess \\
\hline Other bias & Unclear risk & Insufficient information available to assess \\
\hline Implementation Integrity & Unclear risk & Insufficient information available to assess \\
\hline $\begin{array}{l}\text { Blinding of participants and personnel } \\
\text { (performance bias) } \\
\text { All outcomes }\end{array}$ & High risk & $\begin{array}{l}\text { Participants and personnel could not be } \\
\text { blinded, outcome likely to be influenced by } \\
\text { lack of blinding }\end{array}$ \\
\hline $\begin{array}{l}\text { Blinding of outcome assessment (detection } \\
\text { bias) } \\
\text { All outcomes }\end{array}$ & Low risk & $\begin{array}{l}\text { Assessor was not aware of treatment alloca- } \\
\text { tion }\end{array}$ \\
\hline
\end{tabular}


Methods

Participants
Study design: Pilot randomised controlled trial

Setting: Primary care

Diagnosis: International Classification of Diseases (ICD-10) diagnostic criteria for a depressive illness, suffering from a moderate to severe episode (using the Mini-International Neuropsychiatric Interview), and scoring at least 14 on the Hamilton Depression Rating Scale (HDRS17), indicating that they were not in remission

Inclusion criteria: Aged 18-65 years, currently prescribed an antidepressant and had been on this for at least 8 weeks

Exclusion criteria: Secondary care mental health involvement, a recorded diagnosis of personality disorder, an organic brain disorder, alcohol or drug dependency, pregnancy, or learning disability

Age: Inclusion aged $18-65$ years

Gender: Not stated

Ethnicity: Not stated

Country: United Kingdom

Sample size (randomised): Total participants 62, intervention 30, control 32

Interventions

Intervention: Case management

Contains the four elements of collaborative care:

1) a multi-professional approach to patient care: General Practitioner (PCP), graduate mental health worker (CM), psychiatrist (MH specialist)

2) a structured management plan: Patients were prescribed a recommended $A D$ which was monitored by the CM who recommended an

increase in dosage to the PCP, where appropriate and minimal supportive counselling was provided throughout

3 ) scheduled patient follow-ups: 6 contacts in 16 weeks (face-to-face at weeks 1, 4 and

16 and telephone at weeks 2, 6 and 10)

4) enhanced inter-professional communication: CM recommended medication dose changes at weeks 4 and 10 where appropriate. CMs received weekly supervision from MH specialist, who was also available for telephone consultation when needed

Control: Treatment as usual enhanced as all patients received a prescription for an alternative AD within a week of their baseline assessment and PCPs were instructed to prescribe an $\mathrm{AD}$ of their choice, in line with NICE guidelines

Outcomes

Depression (BDI): 3, 6 months

Medication use: 6 months

Satisfaction: 6 months

Notes

AD: antidepressant; BDI: Beck Depression Inventory; CM: case manager; $\mathrm{MH}$ : mental health; NICE: National Institute for Clinical Excellence; PCP: primary care provider

Risk of bias

Bias

Authors' judgement

Support for judgement

Random sequence generation (selection Unclear risk

Insufficient information available to assess bias) 
McMahon 2007 (Continued)

\begin{tabular}{|c|c|c|}
\hline Allocation concealment (selection bias) & Low risk & Central allocation by independent person \\
\hline $\begin{array}{l}\text { Incomplete outcome data (attrition bias) } \\
\text { All outcomes }\end{array}$ & Unclear risk & $\begin{array}{l}\text { Short-term loss to follow-up based on pri- } \\
\text { mary depression outcome (BDI) was: over- } \\
\text { all } 26 / 62(42 \%), 11 / 30(37 \%) \text { interven- } \\
\text { tion and } 15 / 32(47 \%) \text { control. Reasons } \\
\text { for loss to follow-up not sufficiently pro- } \\
\text { vided. Intention-to-treat analysis reported, } \\
\text { with last-observation-carried-forward used } \\
\text { to manage missing data }\end{array}$ \\
\hline Selective reporting (reporting bias) & Unclear risk & Insufficient information available to assess \\
\hline Other bias & Unclear risk & Insufficient information available to assess \\
\hline Implementation Integrity & Unclear risk & Insufficient information available to assess \\
\hline $\begin{array}{l}\text { Blinding of participants and personnel } \\
\text { (performance bias) } \\
\text { All outcomes }\end{array}$ & High risk & $\begin{array}{l}\text { Participants and personnel could not be } \\
\text { blinded, outcome likely to be influenced by } \\
\text { lack of blinding }\end{array}$ \\
\hline $\begin{array}{l}\text { Blinding of outcome assessment (detection } \\
\text { bias) } \\
\text { All outcomes }\end{array}$ & Low risk & $\begin{array}{l}\text { Assessor was not aware of treatment alloca- } \\
\text { tion }\end{array}$ \\
\hline
\end{tabular}

Oslin 2003

Methods

Participants
Study design: Cluster-randomised controlled trial

Setting: Primary care

Diagnosis: Signs and symptoms of a depressive disorder (major depression, dysthymia, or persistent minor depression). Major depression and dysthymia were determined using Diagnostic Statistical Manual (DSM-IV) diagnostic criteria with inclusive strategies for the evaluation of symptoms in patients with medical illness using the MINI psychiatric interview. Persistent minor depression is defined as having DSM provisional criteria for minor depression for a minimum of 4 weeks. The baseline assessment instruments completed included the Mini-International Neuropsychiatric Interview (MINI) modules for depression and anxiety disorders and a scripted version of the Hamilton Rating scale for Depression (HDRS-24)

Inclusion criteria: 18 years or older, male or female, meet criteria for at-risk drinking as defined by drinking more than 21 standard drinks per week ( 14 for women or those older than age 65 ), or binge drinking (> 3 binges in 3 months), or positive CAGE responses combined with any drinking

Exclusion criteria: Active suicidal ideation, regular use of illicit substances, current hallucinations and delusions or a history of a primary psychotic disorder, a history of mania or hypomania, and having a high potential for alcohol withdrawal symptoms as indicated by a score more than 14 on the Alcohol Dependence Scale (ADS)

Age: Mean 61.6 years 
Oslin 2003 (Continued)

Gender: $4 \%$ female

Ethnicity: $50 \%$ white

Country: United States

Sample size (randomised): Total clusters 37, intervention not stated, control not stated;

Total participants 97, intervention 46, control 51

Interventions

Intervention: Telephone disease management

Contains the four elements of collaborative care:

1) a multi-professional approach to patient care: Primary physician (PCP), nurse (CM) , psychiatrist (MH specialist)

2) a structured management plan: CMs developed a treatment plan, monitored outcomes and adverse effects, assessed and encouraged adherence and offered support and education. Contacts were manualised for both depression and at-risk drinking. Where indicated patients were prescribed an AD following AHRQ treatment guidelines. Nonresponse at 6 and 12 weeks resulted in re-evaluation of the treatment plan, to intensify or enhance treatment. For those with at-risk drinking CMs monitored outcomes and used motivational skills to review individual goals and the risks and benefits of drinking using a workbook that was mailed to the patient after each visit. Non-response at 4 months resulted in a recommendation for referral to the Addiction Recovery Unit being made to the patient and PCP

3) scheduled patient follow-ups: Assessment followed by 7 telephone calls in 24 weeks $(1,3,6,9,12,18$ and 24 weeks)

4) enhanced inter-professional communication: CMs acted as physician extender giving behavioural health, medication and referral recommendations to the PCP. CM had weekly supervision with $\mathrm{MH}$ specialist who was also available for consultation

Control: Treatment as usual enhanced as PCPs were educated about existing treatment guidelines, patients attending clinics were screened and PCPs were provided with written diagnostic information for patients and encouraged to refer patients to the behavioural health clinic

Outcomes

Depression (Response to treatment - depression or alcohol): 4 months

Notes

AD: antidepressant; $\mathrm{CM}$ : case manager; $\mathrm{MH}$ : mental health; PCP: primary care provider; AHRQ: Agency for Healthcare Research and Quality

\section{Risk of bias}

Bias

Authors' judgement

Support for judgement

Random sequence generation (selection Unclear risk

Insufficient information available to assess bias)

\begin{tabular}{l|l|l} 
Allocation concealment (selection bias) & Unclear risk & Insufficient information available to assess \\
\hline $\begin{array}{l}\text { Incomplete outcome data (attrition bias) } \\
\text { All outcomes }\end{array}$ & Unclear risk & $\begin{array}{l}\text { Short-term loss to follow-up based on pri- } \\
\text { mary depression outcome (Response to } \\
\text { treatment - depression or alcohol) was: }\end{array}$ \\
& & $\begin{array}{l}\text { overall 23/97(24\%), } 11 / 46(24 \%) \text { inter- } \\
\text { vention and } 12 / 51(24 \%) \text { control. Reasons }\end{array}$
\end{tabular}




\begin{tabular}{l|l|l} 
& & $\begin{array}{c}\text { for loss to follow-up not provided across } \\
\text { groups. Intention-to-treat analysis not re- } \\
\text { ported, unclear methods to manage miss- } \\
\text { ing data }\end{array}$ \\
\hline $\begin{array}{l}\text { Selective reporting (reporting bias) } \\
\text { Other bias }\end{array}$ & Unclear risk & Insufficient information available to assess \\
\hline $\begin{array}{l}\text { Implementation Integrity } \\
\text { Blinding of participants and personnel } \\
\text { (performance bias) } \\
\text { All outcomes }\end{array}$ & Unclear risk risk & Insufficient information available to assess \\
\hline $\begin{array}{l}\text { Blinding of outcome assessment (detection } \\
\text { bias) } \\
\text { All outcomes }\end{array}$ & Unclear risk & Insufficient information available to assess \\
\hline
\end{tabular}

\section{Patel 2010}

Methods

Participants
Study design: Cluster-randomised controlled trial

Setting: Primary care

Diagnosis: Common mental disorder assessed using a score of over 5 on the General Health Questionnaire (GHQ)

Inclusion criteria: Age $>17$ years, not requiring urgent medical attention, not already screened in the previous 2 weeks; and not already receiving the intervention. Those who fulfil the following criteria also invited to participate in the outcome evaluation of the trial: resident in Goa for the subsequent 12 months; speak one of the three primary study languages (Konkani, Marathi, English)

Exclusion criteria: Do not suffer from a serious impairment (hearing, speech, cognition) which interferes with participation in an interview

Age: Mean 46.3 (SD 13.3) years

Gender: $83 \%$ female

Ethnicity: 96\% Indian

Country: India

Sample size (randomised): Total clusters 24, intervention 12, control 12; Total participants 2796, intervention 1360, control 1436

Interventions
Intervention: Collaborative stepped care

Contains the four elements of collaborative care:

1) a multi-professional approach to patient care: Physician/General Practitioners (PCP) , lay health counsellor (CM), psychiatrist (MH specialist)

2) a structured management plan: Step 1: Psychoeducation including strategies to alleviate symptoms, e.g. breathing exercises for anxiety and scheduling activities for depression. Step 2: Management of moderate or severe cases included ADs or interpersonal psychotherapy (IPT) and adherence was encouraged and information provided on social/ 
Patel 2010 (Continued)

\begin{tabular}{|c|c|c|}
\hline & \multicolumn{2}{|c|}{$\begin{array}{l}\text { welfare organisations when needed. Step 3: Non-response patients offered AD and IPT } \\
\text { and adherence management. Step 4: Continue existing treatments and refer to clinical } \\
\text { specialist } \\
\text { 3) scheduled patient follow-ups: IPT: minimum of } 6 \text { sessions, with an optimum of } 8 \\
\text { and maximum of } 12 \\
\text { 4) enhanced inter-professional communication: CM collaborated closely with PCP and } \\
\text { MH specialist, PCP could request a patient consultation with the MH specialist if nec- } \\
\text { essary. MH specialists visited the practice once a month and were available for telephone } \\
\text { consultation } \\
\text { Control: Treatment as usual enhanced as PCP received screening results and were given } \\
\text { the treatment manual that provided information about commonly available drugs and } \\
\text { their side-effects and costs }\end{array}$} \\
\hline Outcomes & \multicolumn{2}{|c|}{ Depression (ICD-10 recovery): 2, 6, 12 months } \\
\hline Notes & \multicolumn{2}{|c|}{$\begin{array}{l}\text { AD: antidepressant; CM: case manager; IPT: interpersonal psychotherapy treatment; } \\
\text { MH: mental health; PCP: primary care provider }\end{array}$} \\
\hline \multicolumn{3}{|l|}{ Risk of bias } \\
\hline Bias & Authors' judgement & Support for judgement \\
\hline $\begin{array}{l}\text { Random sequence generation (selection } \\
\text { bias) }\end{array}$ & Low risk & Computer generated cluster randomisation \\
\hline Allocation concealment (selection bias) & Unclear risk & $\begin{array}{l}\text { Clinics were centrally allocated by an indi- } \\
\text { vidual not involved in recruitment, those } \\
\text { involved in patient recruitment may have } \\
\text { been aware of clinic allocation }\end{array}$ \\
\hline $\begin{array}{l}\text { Incomplete outcome data (attrition bias) } \\
\text { All outcomes }\end{array}$ & Low risk & $\begin{array}{l}\text { Short-term loss to follow-up based on pri- } \\
\text { mary depression outcome (ICD10 recov- } \\
\text { ery) was: overall } 281 / 2242 \text { ( } 13 \%) \text {, } 154 / \\
1098(14 \%) \text { intervention and } 127 / 1144 \\
(11 \%) \text { control. Reasons for loss to follow- } \\
\text { up provided, with similar reasons across } \\
\text { groups. Used intention-to-treat analysis }\end{array}$ \\
\hline Selective reporting (reporting bias) & Low risk & $\begin{array}{l}\text { Protocol available and all prespecified out- } \\
\text { comes reported }\end{array}$ \\
\hline Other bias & Unclear risk & Insufficient information available to assess \\
\hline Implementation Integrity & Low risk & $\begin{array}{l}\text { Attempts were made to assess implemen- } \\
\text { tation integrity (e.g. direct observation or } \\
\text { rating of tapes) }\end{array}$ \\
\hline
\end{tabular}

Collaborative care for depression and anxiety problems (Review)

Copyright (־ 2012 The Cochrane Collaboration. Published by John Wiley \& Sons, Ltd. 
Patel 2010 (Continued)

Blinding of participants and personnel High risk (performance bias)

All outcomes
Participants and personnel could not be blinded, outcome likely to be influenced by lack of blinding

Blinding of outcome assessment (detection Low risk bias)

All outcomes

Piette 2011

Methods

Participants

Interventions
Study design: Randomised controlled trial

Setting: Primary care

Diagnosis: Depression assessed by PHQ-9 score of greater than 11 and BDI score of over 14

Inclusion criteria: At least 21 years old, diagnosis of type 2 diabetes and using antihyperglycaemic medication

Exclusion criteria: Bipolar disorder or schizophrenia, or in active treatment for another serious illness such as severe heart failure, severe chronic obstructive pulmonary disease, or end-stage renal disease. Patients using antidepressant medication at the time of the screening were excluded if they reported a change in the prior 30 days in either their antidepressant medication or the physician prescribing their antidepressants, unable to walk either 1 block or 10 minutes without rest, scored $<21$ on the Short Orientation Memory Concentration Test, or they reported drug or alcohol problems during the prior 3 months as measured by a modified version of the CAGE questionnaire

Age: Mean 56 (SD 10.1) years

Gender: $52 \%$ female

Ethnicity: 84\% White

Country: United States

Sample size (randomised): Total participants 339, intervention 172, control 167

Intervention: Telephone CBT

Contains the four elements of collaborative care:

1) a multi-professional approach to patient care: Primary care provider (PCP), $\mathrm{MH} /$ primary care nurses (CM), CBT therapist ( $\mathrm{MH}$ specialist)

2) a structured management plan: Telephone CBT focused on patients' depressive symptoms, introduced a walking programme, and emphasised the links between depression, physical activity, and diabetes outcomes. CMs and patients used a manual to guide sessions and monitored each week's CBT goals. CMs monitored patients' depressive symptoms and their activity levels. Patient manuals were used to record homework exercises and monitor progress. PCPs were informed of any: suicidal ideation, discontinuation of ADs, persistent elevated depressive symptoms, need for a prescription refill. Additional contacts to discuss patients other health problems were at the CMs discretion 3) scheduled patient follow-ups: Acute $=12$ weekly telephone CBT sessions, maintenance = 9 monthly booster sessions

4) enhanced inter-professional communication: PCPs received written diagnosis detail after assessment and every 3 months thereafter. PCPs were alerted by fax and telephone in the event of any: suicidal ideation, discontinuation of $\mathrm{ADs}$, persistent elevated depressive 
Piette 2011 (Continued)

\begin{tabular}{|c|c|c|}
\hline & \multicolumn{2}{|c|}{$\begin{array}{l}\text { symptoms, or need for a prescription refill. CMs received weekly group supervision from } \\
\text { the MH specialist } \\
\text { Control: Treatment as usual enhanced as patients received a self-help book on CBT } \\
\text { for depression, educational materials about depression and walking and diabetes, and a } \\
\text { list of local resources for depression. With permission PCPs were notified about their } \\
\text { depression scores }\end{array}$} \\
\hline Outcomes & \multicolumn{2}{|c|}{$\begin{array}{l}\text { Depression (BDI): } 12 \text { months } \\
\text { Medication use: } 12 \text { months } \\
\text { Quality of Life (mental and physical health): } 12 \text { months }\end{array}$} \\
\hline Notes & \multicolumn{2}{|c|}{$\begin{array}{l}\text { AD: antidepressant; BDI: Beck Depression Inventory; CM: case manager; } \mathrm{MH} \text { : mental } \\
\text { health; PCP: primary care provider; PHQ-9: Patient Health Questionnaire }\end{array}$} \\
\hline \multicolumn{3}{|l|}{ Risk of bias } \\
\hline Bias & Authors' judgement & Support for judgement \\
\hline $\begin{array}{l}\text { Random sequence generation (selection } \\
\text { bias) }\end{array}$ & Low risk & Random numbers table \\
\hline Allocation concealment (selection bias) & Low risk & Sealed envelopes \\
\hline $\begin{array}{l}\text { Incomplete outcome data (attrition bias) } \\
\text { All outcomes }\end{array}$ & Unclear risk & $\begin{array}{l}\text { Short-term loss to follow-up based on pri- } \\
\text { mary depression outcome (BDI mean) was: } \\
\text { overall } 48 / 339(14 \%), 27 / 172(16 \%) \text { in- } \\
\text { tervention and } 21 / 167(13 \%) \text { control. Rea- } \\
\text { sons for loss to follow-up not provided. } \\
\text { Used intention-to-treat analysis }\end{array}$ \\
\hline Selective reporting (reporting bias) & Unclear risk & Insufficient information available to assess \\
\hline Other bias & Unclear risk & Insufficient information available to assess \\
\hline Implementation Integrity & Low risk & $\begin{array}{l}\text { Attempts were made to assess implemen- } \\
\text { tation integrity (e.g. direct observation or } \\
\text { rating of tapes) }\end{array}$ \\
\hline $\begin{array}{l}\text { Blinding of participants and personnel } \\
\text { (performance bias) } \\
\text { All outcomes }\end{array}$ & High risk & $\begin{array}{l}\text { Participants and personnel could not be } \\
\text { blinded, outcome likely to be influenced by } \\
\text { lack of blinding }\end{array}$ \\
\hline $\begin{array}{l}\text { Blinding of outcome assessment (detection } \\
\text { bias) } \\
\text { All outcomes }\end{array}$ & Unclear risk & Insufficient information available to assess \\
\hline
\end{tabular}

Collaborative care for depression and anxiety problems (Review) 
Pyne 2011

Methods

Participants
Study design: Randomised controlled trial

Setting: Specialist

Diagnosis: PHQ-9 depression score of 10 or higher

Inclusion criteria: Current treatment in the Veteran Affairs HIV clinic

Exclusion criteria: No access to a telephone, current acute suicidal ideation, significant cognitive impairment and history of bipolar disorder or schizophrenia

Age: Mean 49.8 years

Gender: 3\% female

Ethnicity: 63\% African American

Country: United States

Sample size (randomised): Total participants 276 (249), intervention 138 (123), control 138 (126)
Intervention: Stepped care (HITIDES)

Contains the four elements of collaborative care:

1) a multi-professional approach to patient care: HIV or mental health clinician (PCP), nurse (CM), pharmacist/psychiatrist ( $\mathrm{MH}$ specialist)

2) a structured management plan: CMs, using written scripts, delivered education and activation, assessment of treatment barriers and solutions, depression and substance abuse monitoring, and instruction in self-management. The 5-step model included the following plus CM monitoring: (1) watchful waiting, (2) counselling or guideline pharmacotherapy, (3) review by pharmacist, (4) combination pharmacotherapy and counselling, and (5) referral to specialty mental health

3) scheduled patient follow-ups: depended on response. Acute = every 2 weeks (until $50 \%$ reduction in depression score), watchful waiting or continuation $=$ every 4 weeks (for 2 months after maintaining remission or 6 months after maintaining a $50 \%$ decrease in depression score)

4) enhanced inter-professional communication: CMs communicated with PCPs via electronic medical record progress notes. CMs communicated with $\mathrm{MH}$ specialist once a week and as needed by telephone or in-person and made treatment recommendations to PCPs

Control: Treatment as usual enhanced as all HIV health care providers received 1 hour of HIV and depression training and were informed of depression scores. Specialty mental health referral procedures were reviewed

Outcomes

Depression (HSCL-20): 6, 12 months

Medication use: 6, 12 months

Quality of Life (mental and physical health): 6, 12 months

Notes

CM: case manager; HSCL: Hopkins Symptom Checklist; MH: mental health; PCP: primary care provider; PHQ-9: Patient Health Questionnaire

\section{Risk of bias}

\section{Bias}

Random sequence generation (selection Low risk bias)
Support for judgement

Computer generated 
Pyne 2011 (Continued)

\begin{tabular}{|c|c|c|}
\hline Allocation concealment (selection bias) & Unclear risk & Envelopes labelled by patient number \\
\hline $\begin{array}{l}\text { Incomplete outcome data (attrition bias) } \\
\text { All outcomes }\end{array}$ & Low risk & $\begin{array}{l}\text { Short-term loss to follow-up based on pri- } \\
\text { mary depression outcome (HSCL-20 re- } \\
\text { sponse } 50 \% \text { decrease) was: overall 50/276 } \\
(18 \%), 29 / 138(21 \%) \text { intervention and } 21 / \\
138(15 \%) \text { control. Reasons for loss to } \\
\text { follow-up provided, with similar reasons } \\
\text { across groups. Used intention-to-treat anal- } \\
\text { ysis }\end{array}$ \\
\hline Selective reporting (reporting bias) & Unclear risk & Insufficient information available to assess \\
\hline Other bias & Unclear risk & Insufficient information available to assess \\
\hline Implementation Integrity & Unclear risk & Insufficient information available to assess \\
\hline $\begin{array}{l}\text { Blinding of participants and personnel } \\
\text { (performance bias) } \\
\text { All outcomes }\end{array}$ & High risk & $\begin{array}{l}\text { Participants and personnel could not be } \\
\text { blinded, outcome likely to be influenced by } \\
\text { lack of blinding }\end{array}$ \\
\hline $\begin{array}{l}\text { Blinding of outcome assessment (detection } \\
\text { bias) } \\
\text { All outcomes }\end{array}$ & Low risk & $\begin{array}{l}\text { Assessor was not aware of treatment alloca- } \\
\text { tion }\end{array}$ \\
\hline
\end{tabular}

\section{Richards 2008a}

\begin{tabular}{ll}
\hline Methods & Study design: Cluster-randomised controlled trial \\
\hline Participants & Setting: Primary care \\
& Diagnosis: Diagnosed as depressed by a General Practitioner, confirmed by a score of $\geq$ \\
& 5 on the depression section of the Standard Clinical Interview for DSM-IV (SCID) \\
& Inclusion criteria: Patients aged over 18 with a newly identified episode of major depres- \\
& sion, defined as a current episode of GP-initiated treatment of not more than 1 months \\
& duration \\
& Exclusion criteria: Postnatal, bereavement or physical causes for depression, active suicidal \\
plans and primary drug or alcohol dependence & Age: Mean 42.2 years \\
Gender: $78 \%$ female & Ethnicity: $85 \%$ white \\
Country: United Kingdom & Sample size (randomised): Total clusters 24, intervention 12, control 12; Total partici- \\
pants 76, intervention 41, control 35 \\
\hline
\end{tabular}
care $\mathrm{MH}$ workers $(\mathrm{CM})$, psychiatrist/psychological therapist ( $\mathrm{MH}$ specialist) 


\begin{tabular}{|c|c|c|}
\hline & \multicolumn{2}{|c|}{$\begin{array}{l}\text { 2) a structured management plan: Structured management plan of medication support } \\
\text { and behavioural activation } \\
\text { 3) scheduled patient follow-ups: } 10 \text { in } 3 \text { months (initial face-to-face then weekly for } 5 \\
\text { weeks, then fortnightly predominantly telephone calls) } \\
\text { 4) enhanced inter-professional communication: Three levels of communication: Level } \\
\text { 1: treatment plan entered into medical record and brief record after each contact where } \\
\text { patient was progressing/engaging satisfactorily, Level 2: CMs informed PCPs of changes } \\
\text { to treatment plan by specific note, Level 3: CMs communicated in-person or by telephone } \\
\text { with PCP for urgent issues. CMs had weekly telephone supervision with MH specialists } \\
\text { Control: Treatment as usual }\end{array}$} \\
\hline Outcomes & \multicolumn{2}{|c|}{$\begin{array}{l}\text { Depression (PHQ-9): } 3 \text { months } \\
\text { Quality of Life (mental and physical health): } 3 \text { months }\end{array}$} \\
\hline Notes & \multicolumn{2}{|c|}{$\begin{array}{l}\text { CM: case manager; DSM-IV: Diagnostic and Statistical Manual fourth edition; MH: } \\
\text { mental health; PCP: primary care provider; PHQ-9: Patient Health Questionnaire }\end{array}$} \\
\hline \multicolumn{3}{|l|}{ Risk of bias } \\
\hline Bias & Authors' judgement & Support for judgement \\
\hline $\begin{array}{l}\text { Random sequence generation (selection } \\
\text { bias) }\end{array}$ & Low risk & Computer generated \\
\hline Allocation concealment (selection bias) & Unclear risk & $\begin{array}{l}\text { Clusters (PCP practice) were centrally al- } \\
\text { located by independent service. PCPs were } \\
\text { not informed of their allocated group }\end{array}$ \\
\hline $\begin{array}{l}\text { Incomplete outcome data (attrition bias) } \\
\text { All outcomes }\end{array}$ & Low risk & $\begin{array}{l}\text { Short-term loss to follow-up based on pri- } \\
\text { mary depression outcome (PHQ-9) was: } \\
\text { overall } 14 / 76(18 \%), 6 / 41(15 \%) \text { interven- } \\
\text { tion and } 8 / 35(23 \%) \text { control. Reasons for } \\
\text { loss to follow-up provided, with similar rea- } \\
\text { sons across groups. Used intention-to-treat } \\
\text { analysis }\end{array}$ \\
\hline Selective reporting (reporting bias) & Low risk & $\begin{array}{l}\text { Protocol available and all prespecified out- } \\
\text { comes reported }\end{array}$ \\
\hline Other bias & Low risk & $\begin{array}{l}\text { The study appears free of other sources of } \\
\text { bias }\end{array}$ \\
\hline Implementation Integrity & Unclear risk & Insufficient information available to assess \\
\hline $\begin{array}{l}\text { Blinding of participants and personnel } \\
\text { (performance bias) } \\
\text { All outcomes }\end{array}$ & High risk & $\begin{array}{l}\text { Participants and personnel could not be } \\
\text { blinded, outcome likely to be influenced by } \\
\text { lack of blinding }\end{array}$ \\
\hline
\end{tabular}


Richards 2008a (Continued)

Blinding of outcome assessment (detection Low risk bias)

All outcomes
Assessor was not aware of treatment allocation

Richards 2008b

Methods

Participants
Study design: Randomised control trial (within cluster randomised trial)

Setting: Primary care

Diagnosis: Diagnosed as depressed by a General Practitioner, confirmed by a score of $\geq$ 5 on the depression section of the Standard Clinical Interview for DSM-IV (SCID)

Inclusion criteria: Patients aged over 18 with a newly identified episode of major depression, defined as a current episode of GP-initiated treatment of not more than 1 months duration

Exclusion criteria: Postnatal, bereavement or physical causes for depression, active suicidal plans and primary drug or alcohol dependence

Age: Mean 42.8 years

Gender: $77 \%$ female

Ethnicity: 90\% white

Country: United Kingdom

Sample size (randomised): Total participants 79, intervention 41, control 38
Intervention: Collaborative care

Contains the four elements of collaborative care:

1) a multi-professional approach to patient care: General practitioner (PCP), primary care $\mathrm{MH}$ workers $(\mathrm{CM})$, psychiatrist/psychological therapist (MH specialist)

2) a structured management plan: Structured management plan of medication support and behavioural activation

3) scheduled patient follow-ups: 10 in 3 months (initial face-to-face then weekly for 5 weeks, then fortnightly predominantly telephone calls)

4) enhanced inter-professional communication: Three levels of communication: Level 1: treatment plan entered into medical record and brief record after each contact where patient was progressing/engaging satisfactorily, Level 2: CMs informed PCPs of changes to treatment plan by specific note, Level 3: CMs communicated in-person or by telephone with PCP for urgent issues. CMs had weekly telephone supervision with $\mathrm{MH}$ specialists Control: Treatment as usual

Outcomes

Depression (PHQ-9): 3 months

Quality of Life (mental and physical health): 3 months

Notes

CM: case manager; DSM-IV: Diagnostic and Statistical Manual fourth edition; MH: mental health; PCP: primary care provider; PHQ-9: Patient Health Questionnaire

\section{Risk of bias}


Richards 2008b (Continued)

\begin{tabular}{|c|c|c|}
\hline $\begin{array}{l}\text { Random sequence generation (selection } \\
\text { bias) }\end{array}$ & Low risk & Computer generated \\
\hline Allocation concealment (selection bias) & Low risk & $\begin{array}{l}\text { Within the treatment cluster group pa- } \\
\text { tients were centrally allocated by an inde- } \\
\text { pendent service }\end{array}$ \\
\hline $\begin{array}{l}\text { Incomplete outcome data (attrition bias) } \\
\text { All outcomes }\end{array}$ & Low risk & $\begin{array}{l}\text { Short-term loss to follow-up based on pri- } \\
\text { mary depression outcome (PHQ-9) was: } \\
\text { overall 10/79 (13\%), 6/41 (15\%) interven- } \\
\text { tion and } 4 / 38(11 \%) \text { control. Reasons for } \\
\text { loss to follow-up provided, with similar rea- } \\
\text { sons across groups. Used intention-to-treat } \\
\text { analysis }\end{array}$ \\
\hline Selective reporting (reporting bias) & Low risk & $\begin{array}{l}\text { Protocol available and all prespecified out- } \\
\text { comes reported }\end{array}$ \\
\hline Other bias & Low risk & $\begin{array}{l}\text { The study appears free of other sources of } \\
\text { bias }\end{array}$ \\
\hline Implementation Integrity & Unclear risk & $\begin{array}{l}\text { Implementation integrity not assessed } \\
\text { prior to outcome assessment, subsequent } \\
\text { analyses demonstrate good integrity/adher- } \\
\text { ence }\end{array}$ \\
\hline $\begin{array}{l}\text { Blinding of participants and personnel } \\
\text { (performance bias) } \\
\text { All outcomes }\end{array}$ & High risk & $\begin{array}{l}\text { Participants and personnel could not be } \\
\text { blinded, outcome likely to be influenced by } \\
\text { lack of blinding }\end{array}$ \\
\hline $\begin{array}{l}\text { Blinding of outcome assessment (detection } \\
\text { bias) } \\
\text { All outcomes }\end{array}$ & Low risk & $\begin{array}{l}\text { Assessor was not aware of treatment alloca- } \\
\text { tion }\end{array}$ \\
\hline
\end{tabular}

Richards 2012

Methods

Participants
Study design: Cluster-randomised controlled trial

Setting: Primary care

Diagnosis: Depression assessed using the Clinical Interview Schedule (CIS-R)

Inclusion criteria: 18 years and above and who are not currently receiving treatment for depression from specialist mental health services. Also included patients suffering from peri- or postnatal depression, with either co-morbid physical illness or co-morbid nonpsychotic functional disorders, such as anxiety. In line with the pragmatic nature of this trial, we will reflect usual GP care and participants will be eligible to participate whether they are in receipt of antidepressant medication or not

Exclusion Criteria: Patients whose risk of suicide is sufficiently acute to demand immediate management by a specialist mental health crisis team. Patients with psychosis; both 
Richards 2012 (Continued)

type I and type II bi-polar disorder, patients where the low mood is better explained by the death of someone close to them and patients whose primary presenting problem is alcohol or drug abuse. Patients who are currently receiving specialist treatment for their depression will also be excluded

Age: Mean 44.8 (SD 13.3) years

Gender: $72 \%$ female

Ethnicity: 85\% white

Country: United Kingdom

Sample size (randomised): Total clusters 51, intervention 24, control 27; Total participants 581, intervention 276, control 305

Interventions

Intervention: Collaborative care

Contains the four elements of collaborative care:

1) a multi-professional approach to patient care: General practitioner (PCP), primary care $\mathrm{MH}$ workers $(\mathrm{CM})$, psychiatrist/psychological therapist ( $\mathrm{MH}$ specialist)

2) a structured management plan: Involved a structured management plan including education about depression, medication management, behavioural activation and relapse prevention. CMs reinforce the information given by PCP and help problem solve any difficulties with medication concordance. Behavioural activation focused on reducing avoidance and increasing activity. Relapse prevention involved the development of individualised recovery plans to identify symptoms and encourage reinstating pharmacological and psychological depression management strategies

3) scheduled patient follow-ups: 6-12 telephone and face-to-face contacts in 14 weeks (initial face-to-face then weekly for 5 weeks, then fortnightly predominantly telephone calls)

4) enhanced inter-professional communication: CMs helped PCPs and patients problem solve any difficulties with medication. CMs receive weekly supervision from $\mathrm{MH}$ specialists

Control: Treatment as usual

Outcomes

Depression (PHQ-9): 4, 12 months

Quality of Life (mental and physical health): 4, 12 months

Satisfaction: 4, 12 months

Notes

CM: case manager; GP: general practitioner; $\mathrm{MH}$ : mental health; PCP: primary care provider; PHQ-9: Patient Health Questionnaire

Risk of bias

\begin{tabular}{|c|c|c|}
\hline Bias & Authors' judgement & Support for judgement \\
\hline $\begin{array}{l}\text { Random sequence generation (selection } \\
\text { bias) }\end{array}$ & Low risk & $\begin{array}{l}\text { Clusters randomised using a sequence gen- } \\
\text { erated by the trial statistician }\end{array}$ \\
\hline Allocation concealment (selection bias) & Low risk & $\begin{array}{l}\text { Allocation was conducted by central inde- } \\
\text { pendent service and research workers were } \\
\text { blind to cluster allocation and hence to pa- } \\
\text { tient allocation }\end{array}$ \\
\hline
\end{tabular}

Collaborative care for depression and anxiety problems (Review) 
Richards 2012 (Continued)

\begin{tabular}{|l|l|l|}
\hline $\begin{array}{l}\text { Incomplete outcome data (attrition bias) } \\
\text { All outcomes }\end{array}$ & Low risk & $\begin{array}{l}\text { Short-term loss to follow-up based on pri- } \\
\text { mary depression outcome (PHQ-9) was: }\end{array}$ \\
overall 76/581 (13\%), 46/276 (17\%) in- & tervention and 30/305 (10\%) control. Rea- \\
sons for loss to follow-up provided, with \\
similar reasons across groups. Intention to \\
treat not reported
\end{tabular}

Selective reporting (reporting bias) Low risk

Protocol available and all prespecified outcomes reported

\begin{tabular}{|c|c|c|}
\hline Other bias & Low risk & $\begin{array}{l}\text { The study appears free of other sources of } \\
\text { bias }\end{array}$ \\
\hline Implementation Integrity & Unclear risk & $\begin{array}{l}\text { Implementation integrity not assessed } \\
\text { prior to outcome assessment, subsequent } \\
\text { analyses demonstrate good integrity/adher- } \\
\text { ence }\end{array}$ \\
\hline
\end{tabular}

Blinding of participants and personnel High risk (performance bias)

All outcomes

Blinding of outcome assessment (detection Low risk bias)

All outcomes
Participants and personnel could not be blinded, outcome likely to be influenced by lack of blinding

Assessor was not aware of treatment allocation

Rojas 2007

Methods

Participants
Study design: Randomised controlled trial

Setting: Primary care

Diagnosis: Mothers meeting criteria for major depression according to the Diagnostic and Statistical Manual of mental disorders (DSM-IV) were eligible. Any depression detected within 12 months after delivery assessed with the Edinburgh postnatal depression scale (EPDS) scores of over 10 at 2 time points. Used the clinician-administered Mini International Neuropsychiatry Interview (MINI) to ascertain clinical diagnoses Inclusion criteria: Mothers at any stage during their first postnatal year from three clinics in deprived urban areas. Included mothers with an unrecognised and untreated postnatal depression whose symptoms persisted at least for 2 weeks

Exclusion criteria: Women who had received any form of treatment for depression during their current postnatal period, those who were pregnant, or those with psychotic symptoms, serious suicidal risk, history of mania, or alcohol or drug abuse

Age: Mean 26.7 years

Gender: $100 \%$ female

Ethnicity: Not stated

Country: Chile

Sample size (randomised): Total participants 230, intervention 114, control 116 
Rojas 2007 (Continued)

\begin{tabular}{|c|c|c|}
\hline Interventions & \multicolumn{2}{|c|}{$\begin{array}{l}\text { Intervention: Multi-component } \\
\text { Contains the four elements of collaborative care: } \\
\text { 1) a multi-professional approach to patient care: Primary care physician (PCP), non- } \\
\text { professional (CM), psychiatrist (MH specialist) } \\
\text { 2) a structured management plan: The intervention included nurse psychoeducational } \\
\text { groups (problem solving and behavioural activation), CM structured pharmacotherapy } \\
\text { if needed, systematic monitoring of clinical progress and treatment compliance, further } \\
\text { training to doctors, and specialist supervision on a regular basis. CMs monitored atten- } \\
\text { dance and provided support and advice about AD use following a structured format } \\
\text { 3) scheduled patient follow-ups: psychoeducation = } 8 \text { weekly, medication = } 8 \text { in } 6 \text { months } \\
\text { (weeks } 2 \text { and } 4 \text { then monthly) } \\
\text { 4) enhanced inter-professional communication: Nurses had weekly supervision. PCPs } \\
\text { made pharmacotherapy decisions following training and had weekly supervision with } \\
\text { MH specialist } \\
\text { Control: Treatment as usual enhanced as PCPs were informed of the baseline assessment }\end{array}$} \\
\hline Outcomes & \multicolumn{2}{|c|}{$\begin{array}{l}\text { Depression (EPDS): 3, } 6 \text { months } \\
\text { Medication use: 3, } 6 \text { months } \\
\text { Quality of Life (mental and physical health): 3, } 6 \text { months }\end{array}$} \\
\hline Notes & \multicolumn{2}{|c|}{$\begin{array}{l}\text { CM: case manager; DSM-IV: Diagnostic and Statistical Manual fourth edition; MH: } \\
\text { mental health; PCP: primary care provider }\end{array}$} \\
\hline \multicolumn{3}{|l|}{ Risk of bias } \\
\hline Bias & Authors' judgement & Support for judgement \\
\hline $\begin{array}{l}\text { Random sequence generation (selection } \\
\text { bias) }\end{array}$ & Low risk & Computer generated \\
\hline Allocation concealment (selection bias) & Low risk & $\begin{array}{l}\text { Numbered sealed envelopes opened by an } \\
\text { individual not involved in patient recruit- } \\
\text { ment and registered centrally }\end{array}$ \\
\hline $\begin{array}{l}\text { Incomplete outcome data (attrition bias) } \\
\text { All outcomes }\end{array}$ & Unclear risk & $\begin{array}{l}\text { Short-term loss to follow-up based on pri- } \\
\text { mary depression outcome (EPDS) was: } \\
\text { overall } 22 / 230(10 \%), 8 / 114(7 \%) \text { inter- } \\
\text { vention and } 14 / 116(12 \%) \text { control. Rea- } \\
\text { sons for loss to follow-up not provided. } \\
\text { Used intention-to-treat analysis }\end{array}$ \\
\hline Selective reporting (reporting bias) & Unclear risk & Insufficient information available to assess \\
\hline Other bias & Unclear risk & Insufficient information available to assess \\
\hline Implementation Integrity & Unclear risk & Insufficient information available to assess \\
\hline
\end{tabular}

Collaborative care for depression and anxiety problems (Review) 
Rojas 2007 (Continued)

Blinding of participants and personnel High risk (performance bias)

All outcomes
Participants and personnel could not be blinded, outcome likely to be influenced by lack of blinding

Assessor was not aware of treatment allocation

bias)

All outcomes

Rollman 2005

Methods Study design: Randomised controlled trial

Participants

Setting: Primary care

Diagnosis: Anxiety symptoms assessed using the brief self-administered patient questionnaire portion of the PRIME-MD, Diagnostic and Statistical Manual (DSM-IV) criteria for panic or generalised anxiety disorder assessed by PRIME-MD anxiety module. At least moderate levels of anxiety severity as defined by a score of 14 or higher on the 14item structured interview guide for the Hamilton Anxiety Rating Scale (SIGH-A)

Inclusion criteria: Aged 18 to 64, not receiving treatment from a mental health professional, no history of bipolar disorder; and no plans to leave the study practice within the following year

Exclusion criteria: Dementia, psychotic illness, unstable medical condition, 2 or fewer positive responses on the CAGE alcohol screening questionnaire, and language or other communication barriers

Age: Mean 44.2 (SD 10.7) years

Gender: $81 \%$ female

Ethnicity: 95\% Caucasian

Country: United States

Sample size (randomised): Total participants 191, intervention 116, control 75

Interventions

Intervention: Telephone care management

Contains the four elements of collaborative care:

1) a multi-professional approach to patient care: Primary care provider (PCP), nonbehavioural health specialist (CM), psychologist/psychiatrist ( $\mathrm{MH}$ specialist)

2) a structured management plan: CM conducted telephone assessment, provided basic psychoeducation about anxiety, and assessed treatment preferences. Patients chose any combination of the following: (1) a self-management workbook with CM follow-up; (2) a guideline-based trial of anxiolytic pharmacotherapy; or (3) referral to a community mental health specialist. CMs telephoned patients to promote adherence and assess clinical response

3) scheduled patient follow-ups: Acute $=8$ telephone (at 1 week then every two weeks for first 2-4 months), maintenance $=8$ telephone calls (every 1-3 months for up to 12 months)

4) enhanced inter-professional communication: CMs informed PCPs of patient progress and in consultation with $\mathrm{MH}$ specialists recommended specific medication and dose or referral to PCP. CM received weekly supervision from $\mathrm{MH}$ specialists

Control: Treatment as usual enhanced as PCPs were informed of diagnosis by interactive e-mail alert and an electronic letter. The messages encouraged the PCP to follow up 
Rollman 2005 (Continued)

patients to determine whether treatment was required. Patients were also informed of diagnosis and provided with a disorder-specific brochure

\begin{tabular}{ll} 
Outcomes & $\begin{array}{l}\text { Anxiety (SIGH-A): 2, 4, 8, } 12 \text { months } \\
\text { Medication use: } 12 \text { months } \\
\text { Quality of Life (mental and physical health): 4, } 12 \text { months }\end{array}$ \\
\hline Notes & CM: case manager; $\mathrm{MH}$ : mental health; PCP: primary care provider
\end{tabular}

\section{Risk of bias}

\begin{tabular}{|c|c|c|}
\hline Bias & Authors' judgement & Support for judgement \\
\hline $\begin{array}{l}\text { Random sequence generation (selection } \\
\text { bias) }\end{array}$ & Low risk & Computer generated in blocks of 25 or 30 \\
\hline Allocation concealment (selection bias) & Low risk & $\begin{array}{l}\text { Opaque sequentially numbered sealed en- } \\
\text { velopes opened by an individual not in- } \\
\text { volved in patient recruitment }\end{array}$ \\
\hline $\begin{array}{l}\text { Incomplete outcome data (attrition bias) } \\
\text { All outcomes }\end{array}$ & Unclear risk & $\begin{array}{l}\text { Short-term loss to follow-up based on } \\
\text { primary anxiety outcome (SIGH-A) was: } \\
\text { overall } 63 / 191(33 \%), 38 / 116(33 \%) \text { in- } \\
\text { tervention and } 25 / 75(33 \%) \text { control. Rea- } \\
\text { sons for loss to follow-up not provided } \\
\text { across groups. Intention-to-treat analysis } \\
\text { reported, used random regression models } \\
\text { to manage missing data }\end{array}$ \\
\hline Selective reporting (reporting bias) & Low risk & $\begin{array}{l}\text { Protocol available and all prespecified out- } \\
\text { comes reported }\end{array}$ \\
\hline Other bias & Unclear risk & Insufficient information available to assess \\
\hline Implementation Integrity & Unclear risk & Insufficient information available to assess \\
\hline $\begin{array}{l}\text { Blinding of participants and personnel } \\
\text { (performance bias) } \\
\text { All outcomes }\end{array}$ & High risk & $\begin{array}{l}\text { Participants and personnel could not be } \\
\text { blinded, outcome likely to be influenced by } \\
\text { lack of blinding }\end{array}$ \\
\hline $\begin{array}{l}\text { Blinding of outcome assessment (detection } \\
\text { bias) } \\
\text { All outcomes }\end{array}$ & Low risk & $\begin{array}{l}\text { Assessor was not aware of treatment alloca- } \\
\text { tion }\end{array}$ \\
\hline
\end{tabular}


Methods

Participants
Study design: Randomised controlled trial

Setting: Specialist, primary care

Diagnosis: PHQ-9 scores of 10 or greater confirmed the prior PHQ-2 screen results and indicated at least a moderate level of depressive symptoms

Inclusion criteria: Post-CABG (Coronary Artery Bypass Graft) patients, mental competence to provide consent, have no current alcohol dependence or other substance abuse disorder; not be in treatment with a mental health specialist, express actives suicidality, or have a history of psychotic illness or bipolar disorder, be discharged home or to shortterm rehabilitation; and to speak English, have no communication barriers, and have telephone access

Exclusion criteria: Not stated

Age: Mean 64 years

Gender: $61 \%$ female

Ethnicity: 91\% white

Country: United States

Sample size (randomised): Total participants 302, intervention 150, control 152

2) a structured management plan: CM conducted telephone assessment, provided basic education about depression (its impact on cardiac disease, and various self-management strategies) and assessed treatment preferences. Patients were supplied with written educational materials and offered a variety of treatment options: (1) initiation or adjustment of $\mathrm{AD}$; (2) referral to community mental health service; (3) a combination of the above; or (4) watchful-waiting. CMs advised all patients to: (1) get sufficient rest; (2) engage in appropriate exercise and other pleasurable activities; and (3) avoid tobacco, alcohol, and unhealthy foods. CMs sent out a workbook that integrated both a psychotherapeutic and pharmacologic approach to managing depression and worked with patients and PCPs to promote adherence. For non-adherence or non-response after 6 weeks, combined treatment was recommended. For continued non-response CMs recommended referral to $\mathrm{MH}$ services and $\mathrm{CMs}$ monitored attendance and continued to telephone the patient monthly to: (1) monitor mood; (2) relay clinical information to PCP and MH specialist; and (3) promote adherence with follow-up appointments

3) scheduled patient follow-ups: Acute $=4-8$ telephone calls (fortnightly for 2-4 months) , maintenance $=4-8$ (every 1 to 2 months until end of 8 month)

4) enhanced inter-professional communication: CM reported back to the PCP clinical progress, reasons for non-adherence and treatment recommendations via fax, telephone or mail after each case review with $\mathrm{MH}$ specialist. CMs discussed $\mathrm{AD}$ prescriptions with PCP. An end-of-intervention letter was sent to PCP describing current level of depressive symptoms, care preferences, and final treatment recommendations. CMs had weekly case review with $\mathrm{MH}$ specialist

Control: Treatment as usual enhanced as patients and PCPs were informed of diagnosis 
Rollman 2009 (Continued)

Notes
CM: case manager; HRSD: Hamilton Rating Scale for Depression; MH: mental health;
PCP: primary care provider; PHQ-9: Patient Health Questionnaire-9

\section{Risk of bias}

\begin{tabular}{|c|c|c|}
\hline Bias & Authors' judgement & Support for judgement \\
\hline $\begin{array}{l}\text { Random sequence generation (selection } \\
\text { bias) }\end{array}$ & Low risk & $\begin{array}{l}\text { Computer generated in blocks of } 4 \text { using a } \\
1: 1 \text { allocation ratio }\end{array}$ \\
\hline Allocation concealment (selection bias) & Low risk & $\begin{array}{l}\text { Prepared by an individual not involved in } \\
\text { patient recruitment and entered into com- } \\
\text { puter assisted programme and concealed } \\
\text { until after the patient was recruited }\end{array}$ \\
\hline $\begin{array}{l}\text { Incomplete outcome data (attrition bias) } \\
\text { All outcomes }\end{array}$ & Low risk & $\begin{array}{l}\text { Short-term loss to follow-up based on pri- } \\
\text { mary depression outcome ( } \geq 50 \% \text { decline } \\
\text { in HRSD) was: overall } 50 / 302(17 \%), 24 / \\
150(16 \%) \text { intervention and } 26 / 152(17 \%) \\
\text { control. Reasons for loss to follow-up pro- } \\
\text { vided, with similar reasons across groups. } \\
\text { Used intention-to-treat analysis }\end{array}$ \\
\hline Selective reporting (reporting bias) & Low risk & $\begin{array}{l}\text { Protocol available and all prespecified out- } \\
\text { comes reported }\end{array}$ \\
\hline Other bias & Unclear risk & Insufficient information available to assess \\
\hline Implementation Integrity & Unclear risk & Insufficient information available to assess \\
\hline $\begin{array}{l}\text { Blinding of participants and personnel } \\
\text { (performance bias) } \\
\text { All outcomes }\end{array}$ & High risk & $\begin{array}{l}\text { Participants and personnel could not be } \\
\text { blinded, outcome likely to be influenced by } \\
\text { lack of blinding }\end{array}$ \\
\hline $\begin{array}{l}\text { Blinding of outcome assessment (detection } \\
\text { bias) } \\
\text { All outcomes }\end{array}$ & Low risk & $\begin{array}{l}\text { Assessor was not aware of treatment alloca- } \\
\text { tion }\end{array}$ \\
\hline
\end{tabular}

Ross 2008

\begin{tabular}{ll}
\hline Methods & Study design: Cluster-randomised controlled trial \\
\hline Participants & Setting: Primary care \\
& Diagnosis: PHQ scores ranging from 0-16 without a diagnosis of major depression or \\
& other severe axis 1 disorders. Minor depression (those with 2, 3, or 4 Diagnostic and \\
& Statistical Manual depression criteria) and those with distress or depressive symptoms \\
& not meeting minor depression criteria. Measured with the PHQ-9 for depression; the \\
& MINI International Neuropsychiatric Interview modules for mania, psychosis, panic \\
\hline
\end{tabular}


disorder, generalised anxiety disorder (GAD), PTSD, and alcohol abuse/dependence Inclusion criteria: Clinical concern generated by the PCP and on the results of the Behavioural Health Laboratory assessment. Subjects were eligible for inclusion if they were referred by their PCP for a behavioural health concern and did not meet for any exclusion criteria

Exclusion criteria: Current PTSD, panic disorder, alcohol dependence, suicidal ideation, illicit drug use (past year), or if they had a history of or current bipolar or psychotic disorder. Subjects were also excluded if they were being followed by a $\mathrm{MH}$ clinician or if they were currently taking any antidepressants benzodiazepines, antipsychotics, addiction medications, or mood stabilisers

Age: Mean 59.2 (SD 15.9) years

Gender: $7 \%$ female

Ethnicity: $43 \%$ white

Country: United States

Sample size (randomised): Total clusters unclear ( 54 practitioners but randomised by clinic); Total participants 223, intervention 130, control 93

2) a structured management plan: Telephone contacts were manualised and included recommending the $\mathrm{PCP}$ initiated $\mathrm{ADs}$ and $\mathrm{CMs}$ frequent monitoring of adverse effects, adherence and depressive symptoms. CMs also provided support and education about depressive disorders and for any other $\mathrm{MH}$ problems the $\mathrm{CM}$ formulated an appropriate treatment plan which could include referral to specialty care or care management for anxiety

3) scheduled patient follow-ups: 5 calls in 12 weeks (at weeks 2, 4, 6, 9, 12)

4) enhanced inter-professional communication: CMs recommended PCPs initiate ADs and received supervision from $\mathrm{MH}$ specialist

Control: Treatment as usual enhanced as all subjects were assessed by the Behavioural Health Laboratory and PCPs were given a report with suggestions for ongoing monitoring of depressive symptoms and had the option to request referral of patients to a mental health clinic. Patients received a letter following assessment that included selfhelp advice for any significant depression symptoms and encouragement to discuss his or her symptoms with PCP

Outcomes

Depression (PHQ-9): 6 months

Medication use: 6 months

Quality of Life (mental and physical health): 6 months mary care provider; PHQ-9: Patient Health Questionnaire: PTSD: post-traumatic stress disorder

Risk of bias 
Ross 2008 (Continued)

\begin{tabular}{|c|c|c|}
\hline $\begin{array}{l}\text { Random sequence generation (selection } \\
\text { bias) }\end{array}$ & Unclear risk & Insufficient information available to assess \\
\hline Allocation concealment (selection bias) & Unclear risk & Insufficient information available to assess \\
\hline $\begin{array}{l}\text { Incomplete outcome data (attrition bias) } \\
\text { All outcomes }\end{array}$ & Unclear risk & $\begin{array}{l}\text { Short-term loss to follow-up based on pri- } \\
\text { mary depression outcome (PHQ total) was: } \\
\text { overall 59/223 (26\%), 36/130 (28\%) inter- } \\
\text { vention and } 23 / 93(25 \%) \text { control. Reasons } \\
\text { for loss to follow-up provided, with simi- } \\
\text { lar reasons for missing data across groups. } \\
\text { Intention-to-treat analysis reported, no de- } \\
\text { scription of methods to manage missing } \\
\text { data }\end{array}$ \\
\hline Selective reporting (reporting bias) & Unclear risk & Insufficient information available to assess \\
\hline Other bias & Unclear risk & Insufficient information available to assess \\
\hline Implementation Integrity & Unclear risk & Insufficient information available to assess \\
\hline $\begin{array}{l}\text { Blinding of participants and personnel } \\
\text { (performance bias) } \\
\text { All outcomes }\end{array}$ & High risk & $\begin{array}{l}\text { Participants and personnel could not be } \\
\text { blinded, outcome likely to be influenced by } \\
\text { lack of blinding }\end{array}$ \\
\hline
\end{tabular}

Blinding of outcome assessment (detection Unclear risk

Insufficient information available to assess bias)

All outcomes

\section{Rost 2001a}

\begin{tabular}{|c|c|}
\hline Methods & Study design: Cluster-randomised controlled trial \\
\hline Participants & $\begin{array}{l}\text { Setting: Primary care } \\
\text { Diagnosis: Patients screened first-stage positive for depression if they reported on Com- } \\
\text { posite International Diagnostic Interview (CIDI) questions they had experienced } 2 \text { weeks } \\
\text { or more during the last year when they felt sad, empty, depressed, or lost interest in things } \\
\text { they normally enjoyed and reported } 1 \text { week or more of these symptoms during the past } \\
\text { month. Second stage screening: reporting } 5 \text { or more of } 9 \text { criteria for major depression in } \\
\text { the past } 2 \text { weeks on the Inventory to Diagnose Depression. Meeting DSM-III-R criteria } \\
\text { for major depression in the past two weeks. Depression severity measured by a modified } \\
23 \text { item Centre for Epidemiologic Studies depression scale (mCES-D) } \\
\text { Inclusion criteria: Age } 18 \text { or over, not pregnant, breastfeeding or less than } 3 \text { months } \\
\text { post-partum, sufficient literacy in English and cognitive function to complete surveys } \\
\text { requiring 6-month recall, no acute life threatening physical conditions; and access to a } \\
\text { telephone } \\
\text { Exclusion criteria: Depressive symptoms had begun after the loss of a loved one within } \\
\text { the last } 2 \text { months to exclude patients with bereavement. Patients were also excluded if }\end{array}$ \\
\hline
\end{tabular}




\begin{tabular}{|c|c|c|}
\hline & \multicolumn{2}{|c|}{$\begin{array}{l}\text { they noted that they did not intend to receive ongoing care in the clinic during the } \\
\text { next year to target the intervention to patients who could participate in it over time. } \\
\text { Screening positive by self-report for lifetime mania, use of lithium, or current alcohol } \\
\text { dependence } \\
\text { Age: } 42.6 \text { (SD } 13.1 \text { ) years } \\
\text { Gender: } 84 \% \text { female } \\
\text { Ethnicity: } 16 \% \text { ethnic minority } \\
\text { Country: United States } \\
\text { Sample size (randomised): Total clusters } 12 \text {, intervention } 6 \text { ( } 4 \text { urban and } 2 \text { rural), control } \\
6 \text { ( } 4 \text { urban and } 2 \text { rural); Total participants (Recently treated) } 479 \text {, intervention } 239 \text {, } \\
\text { control } 240\end{array}$} \\
\hline Interventions & \multicolumn{2}{|c|}{$\begin{array}{l}\text { Intervention: } \\
\text { Contains the four elements of collaborative care: } \\
\text { 1) a multi-professional approach to patient care: Primary care physician (PCP), nurse } \\
\text { (CM), social worker/psychiatrist (MH specialist) } \\
\text { 2) a structured management plan: CMs assessed patients, evaluated treatment preferences } \\
\text { (pharmacotherapy, psychotherapy, watchful waiting), and addressed barriers to care. A } \\
\text { checklist was then provided to the PCP who then saw the patient. CMs provided written } \\
\text { information on preferred treatment, the homework assignment they had agreed upon, } \\
\text { and the time/place of next CM contact. CMs used a similar protocol to guide subsequent } \\
\text { sessions. With PCP supervision, CMs provided medication samples to patients who } \\
\text { could not afford them } \\
\text { 3) scheduled patient follow-ups: Face-to-face assessment followed by } 5 \text { weekly telephone } \\
\text { or face-to-face contacts with the option of extending the protocol for } 2 \text { additional weeks } \\
\text { 4) enhanced inter-professional communication: CMs provided information from as- } \\
\text { sessment and follow-up to PCPs. PCPs were offered MH specialist consultation. CMs } \\
\text { received supervision from MH specialist (social worker) } \\
\text { Control: Treatment as usual }\end{array}$} \\
\hline Outcomes & \multicolumn{2}{|c|}{$\begin{array}{l}\text { Depression (mCESD): } 6 \text { months } \\
\text { Medication use: } 6 \text { months } \\
\text { Satisfaction: } 6 \text { months }\end{array}$} \\
\hline Notes & \multicolumn{2}{|c|}{$\begin{array}{l}\text { CIDI: Composite International Diagnostic Interview; CM: case manager; } \mathrm{MH} \text { : mental } \\
\text { health; PCP: primary care provider; SD: standard deviation }\end{array}$} \\
\hline \multicolumn{3}{|l|}{ Risk of bias } \\
\hline Bias & Authors' judgement & Support for judgement \\
\hline $\begin{array}{l}\text { Random sequence generation (selection } \\
\text { bias) }\end{array}$ & Low risk & $\begin{array}{l}\text { Clusters were randomised in blocks of } 2 \text {, } \\
\text { with } 1: 1 \text { allocation ratio, using coin-toss }\end{array}$ \\
\hline Allocation concealment (selection bias) & Unclear risk & $\begin{array}{l}\text { Clusters were identified by a number and } \\
\text { paired by the Principle Investigator based } \\
\text { on proportions }\end{array}$ \\
\hline
\end{tabular}




\begin{tabular}{|c|c|c|}
\hline $\begin{array}{l}\text { Incomplete outcome data (attrition bias) } \\
\text { All outcomes }\end{array}$ & Unclear risk & $\begin{array}{l}\text { Short-term loss to follow-up based on pri- } \\
\text { mary depression outcome (mCESD) was: } \\
\text { overall } 26 / 268(10 \%), 12 / 124(10 \%) \text { in- } \\
\text { tervention and } 14 / 144(10 \%) \text { control. Rea- } \\
\text { sons for loss to follow-up not provided. } \\
\text { Used intention-to-treat analysis }\end{array}$ \\
\hline Selective reporting (reporting bias) & Low risk & $\begin{array}{l}\text { Protocol available and all prespecified out- } \\
\text { comes reported }\end{array}$ \\
\hline Other bias & Unclear risk & Insufficient information available to assess \\
\hline Implementation Integrity & Unclear risk & Insufficient information available to assess \\
\hline $\begin{array}{l}\text { Blinding of participants and personnel } \\
\text { (performance bias) } \\
\text { All outcomes }\end{array}$ & High risk & $\begin{array}{l}\text { Participants and personnel could not be } \\
\text { blinded, outcome likely to be influenced by } \\
\text { lack of blinding }\end{array}$ \\
\hline $\begin{array}{l}\text { Blinding of outcome assessment (detection } \\
\text { bias) } \\
\text { All outcomes }\end{array}$ & Low risk & $\begin{array}{l}\text { Assessor was not aware of treatment alloca- } \\
\text { tion }\end{array}$ \\
\hline
\end{tabular}

Rost 2001b

Methods

Study design: Cluster-randomised controlled trial

Participants

Setting: Primary care

Diagnosis: Patients screened first-stage positive for depression if they reported on Composite International Diagnostic Interview (CIDI) questions they had experienced 2 weeks or more during the last year when they felt sad, empty, depressed, or lost interest in things they normally enjoyed and reported 1 week or more of these symptoms during the past month. Second stage screening: reporting 5 or more of 9 criteria for major depression in the past 2 weeks on the Inventory to Diagnose Depression. Meeting DSM-III-R criteria for major depression in the past two weeks. Depression severity measured by a modified 23 item Centre for Epidemiologic Studies depression scale (mCES-D)

Inclusion criteria: Age 18 or over, not pregnant, breastfeeding or less than 3 months post-partum, sufficient literacy in English and cognitive function to complete surveys requiring 6-month recall, no acute life threatening physical conditions; and access to a telephone

Exclusion criteria: Depressive symptoms had begun after the loss of a loved one within the last 2 months to exclude patients with bereavement. Patients were also excluded if they noted that they did not intend to receive ongoing care in the clinic during the next year to target the intervention to patients who could participate in it over time. Screening positive by self-report for lifetime mania, use of lithium, or current alcohol dependence

Age: 42.6 (SD 13.1) years

Gender: $84 \%$ female

Ethnicity: $16 \%$ ethnic minority 
Country: United States

Sample size (randomised): Total clusters 12, intervention 6 ( 4 urban and 2 rural), control 6 (4 urban and 2 rural); Total participants (patients starting new treatment episode) 479 , intervention 239 , control 240

Interventions

Intervention:

Contains the four elements of collaborative care:

1) a multi-professional approach to patient care: Primary care physician (PCP), nurse (CM), social worker/psychiatrist (MH specialist)

2) a structured management plan: CMs assessed patients, evaluated treatment preferences (pharmacotherapy, psychotherapy, watchful waiting), and addressed barriers to care. A checklist was then provided to the PCP who then saw the patient. CMs provided written information on preferred treatment, the homework assignment they had agreed upon, and the time/place of next CM contact. CMs used a similar protocol to guide subsequent sessions. With PCP supervision, CMs provided medication samples to patients who could not afford them

3) scheduled patient follow-ups: Face-to-face assessment followed by 5 weekly telephone or face-to-face contacts with the option of extending the protocol for 2 additional weeks 4) enhanced inter-professional communication: CMs provided information from assessment and follow-p to PCPs. PCPs were offered MH specialist consultation. CMs received supervision from $\mathrm{MH}$ specialist (social worker)

Control: Treatment as usual

$\begin{array}{ll}\text { Outcomes } & \text { Depression (mCESD): } 6 \text { months } \\ & \text { Medication use: } 6 \text { months } \\ & \text { Satisfaction: } 6 \text { months }\end{array}$

Notes

CIDI: Composite International Diagnostic Interview; CM: case manager; $\mathrm{MH}$ : mental health; PCP: primary care provider; SD: standard deviation

\section{Risk of bias}

\begin{tabular}{|c|c|c|}
\hline Bias & Authors' judgement & Support for judgement \\
\hline $\begin{array}{l}\text { Random sequence generation (selection } \\
\text { bias) }\end{array}$ & Low risk & $\begin{array}{l}\text { Clusters were randomised in blocks of } 2 \text {, } \\
\text { with } 1: 1 \text { allocation ratio, using coin-toss }\end{array}$ \\
\hline Allocation concealment (selection bias) & Unclear risk & $\begin{array}{l}\text { Clusters were identified by a number and } \\
\text { paired by the Principle Investigator based } \\
\text { on proportions }\end{array}$ \\
\hline
\end{tabular}

Incomplete outcome data (attrition bias) Unclear risk All outcomes
Short-term loss to follow-up based on primary depression outcome (mCESD) was: overall $22 / 211(10 \%), 18 / 115(16 \%)$ intervention and 4/96 (4\%) control. Reasons for loss to follow-up not provided. Intentionto-treat analysis reported, used random regression analysis to manage missing data 
Rost 2001b (Continued)

\begin{tabular}{|c|c|c|}
\hline Selective reporting (reporting bias) & Low risk & $\begin{array}{l}\text { Protocol available and all prespecified out- } \\
\text { comes reported }\end{array}$ \\
\hline Other bias & Unclear risk & Insufficient information available to assess \\
\hline Implementation Integrity & Unclear risk & Insufficient information available to assess \\
\hline $\begin{array}{l}\text { Blinding of participants and personnel } \\
\text { (performance bias) } \\
\text { All outcomes }\end{array}$ & High risk & $\begin{array}{l}\text { Participants and personnel could not be } \\
\text { blinded, outcome likely to be influenced by } \\
\text { lack of blinding }\end{array}$ \\
\hline $\begin{array}{l}\text { Blinding of outcome assessment (detection } \\
\text { bias) } \\
\text { All outcomes }\end{array}$ & Low risk & $\begin{array}{l}\text { Assessor was not aware of treatment alloca- } \\
\text { tion }\end{array}$ \\
\hline
\end{tabular}

Roy-Byrne 2001

Methods

Participants
Study design: Randomised controlled trial

Setting: Primary care

Diagnosis: Meeting Diagnostic and Statistical Manual (DSM-IV) criteria for Panic Disorder, with at least 1 panic attack in the past month. Assessment included portions of the Composite International Diagnostic Interview (CIDI), modified for DSM-IV, the PDSS, the Anxiety Sensitivity Inventory (ASI), the Fear Questionnaire; and the Centre for Epidemiological Studies Depression Scale (CES-D)

Inclusion criteria: Between age 18 and 65, English-speaking and have a telephone to participate in follow-up assessments

Exclusion criteria: Patients currently receiving psychiatric treatment and patients currently receiving or applying for disability benefits. Potentially life threatening co morbidities (e.g., active suicidal ideation or terminal medical illness) or those that would limit patient participation or adherence (psychosis, current substance abuse, dementia, and pregnancy)

Age: Mean 40.8 (SD 10.3) years

Gender: $57 \%$ female

Ethnicity: 67\% white

Country: United States

Sample size (randomised): Total participants 115, intervention 57, control 58
Intervention: Collaborative care

Contains the four elements of collaborative care:

1) a multi-professional approach to patient care: Primary care physician (PCP), psychiatrist (CM/MH specialist)

2) a structured management plan: CMs assessed and prescribed SSRI (typically paroxetine). Paroxetine was started at $10 \mathrm{mg}$ daily, increased to $20 \mathrm{mg}$ as tolerated in the second week and, if no response was reported by the fourth week and the patient was able to tolerate it, $40 \mathrm{mg}$. Patients also received an educational videotape about panic disorder and an educational pamphlet about ADs and adverse effects. These points were systematically re-emphasised during follow-ups at which CMs addressed negative attitudes 
toward medication, ADs or diagnosis. Patients were encouraged to expose themselves, as tolerated, to any feared and avoided situations

3) scheduled patient follow-ups: 2 face-to-face and 2 calls in 8 weeks then up to 5 calls between 3 and 12 months. Selected patients also seen for up to 3 extra sessions

4) enhanced inter-professional communication: The PCP received a typed consultation note after each CM contact

Control: Treatment as usual enhanced as PCPs were informed of diagnosis

Outcomes
Anxiety (PDSS): 3, 6, 9, 12 months

Medication use: 3, 6, 9, 12 months

Satisfaction: 6,12 months

CIDI: Composite International Diagnostic Interview; CM: case manager; $\mathrm{MH}$ : mental health; PCP: primary care provider; PDSS: Panic Disorder Severity Scale; SSRI: selective serotonin reuptake inhibitor

\section{Risk of bias}

\section{Bias}

Random sequence generation (selection Low risk bias)

\begin{tabular}{|c|c|c|}
\hline Allocation concealment (selection bias) & Unclear risk & Insufficient information available to assess \\
\hline $\begin{array}{l}\text { Incomplete outcome data (attrition bias) } \\
\text { All outcomes }\end{array}$ & Unclear risk & $\begin{array}{l}\text { Short-term loss to follow-up based on pri- } \\
\text { mary depression outcome (PDSS Panic) } \\
\text { was: overall 23/115 (20\%), 12/57 (21\%) } \\
\text { intervention and } 11 / 58(19 \%) \text { control. } \\
\text { Reasons for loss to follow-up not provided. } \\
\text { Used intention-to-treat analysis }\end{array}$ \\
\hline Selective reporting (reporting bias) & Unclear risk & Insufficient information available to assess \\
\hline Other bias & Unclear risk & Insufficient information available to assess \\
\hline Implementation Integrity & Low risk & $\begin{array}{l}\text { Attempts were made to assess implemen- } \\
\text { tation integrity (e.g. direct observation or } \\
\text { rating of tapes) }\end{array}$ \\
\hline $\begin{array}{l}\text { Blinding of participants and personnel } \\
\text { (performance bias) } \\
\text { All outcomes }\end{array}$ & High risk & $\begin{array}{l}\text { Participants and personnel could not be } \\
\text { blinded, outcome likely to be influenced by } \\
\text { lack of blinding }\end{array}$ \\
\hline $\begin{array}{l}\text { Blinding of outcome assessment (detection } \\
\text { bias) } \\
\text { All outcomes }\end{array}$ & Low risk & $\begin{array}{l}\text { Assessor was not aware of treatment alloca- } \\
\text { tion }\end{array}$ \\
\hline
\end{tabular}

\section{Support for judgement}

Random numbers table Authors' judgement

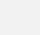


Methods

Participants
Study design: Randomised controlled trial

\section{Setting: Primary care}

Diagnosis: Meeting Diagnostic and Statistical Manual (DSM-IV) criteria for panic disorder with at least 1 panic attack in the prior week. The Composite International Diagnostic Interview (CIDI) used to determine eligibility

Inclusion criteria: Between 18 and 70 years of age, English speaking, access to a telephone, and willing to accept a combined treatment of anti-anxiety medication and CBT

Exclusion criteria: Potentially life threatening co morbidities (i.e. suicidal ideation, terminal medical illness) or those expected to severely limit patient participation or adherence (e.g., psychosis, current substance abuse, dementia, pregnancy). Patients receiving psychiatric disability benefits or those already seeing a psychiatrist or cognitive-behavioural therapist were excluded

Age: Mean 41.2 years

Gender: $67 \%$ female

Ethnicity: 66\% white

Country: United States

Sample size (randomised): Total participants 232, intervention 119, control 113
Interventions
Intervention: CBT

Contains the four elements of collaborative care:

1) a multi-professional approach to patient care: Primary care physician (PCP), graduates (CM), psychiatrist (MH specialist)

2) a structured management plan: Patients received a video about panic disorder and a workbook including education on medication, its management and synergies with CBT. The medication algorithm involved dose titration of typically a Selective Serotonin Reuptake Inhibitor (SSRI) for at least 6 weeks or adjunctive medications (e.g., benzodiazepines). CMs coordinated care and delivered CBT, which targeted panic symptoms but also included modules to address depressive and social anxiety symptoms if required. Follow-up calls monitored clinical status, reinforced medication use and CBT skills, and make further medication recommendations if necessary

3) scheduled patient follow-ups: CBT $=6$ sessions in 3 months ( 3 face-to-face and then telephone if preferred) then 6 brief booster telephone calls at 6-12 week intervals

4) enhanced inter-professional communication: CMs relayed recommendations from MH specialist to PCP. CMs communicated with PCPs using rapid systems of 2-way communication (i.e. telephone, fax, and e-mail). CM received weekly supervision from $\mathrm{MH}$ specialist

Control: Treatment as usual enhanced as PCPs were informed of diagnosis

Outcomes

Anxiety (Composite measure of high end state functioning): 3, 6, 9, 12 months Medication use: 3, 6, 9, 12 months

Quality of Life (mental and physical health): 3, 6, 9, 12 months

Notes

CBT: cognitive behaviour therapy; CIDI: Composite International Diagnostic Interview; CM: case manager; $\mathrm{MH}$ : mental health; PCP: primary care provider 
Roy-Byrne 2005 (Continued)

\begin{tabular}{|c|c|c|}
\hline $\begin{array}{l}\text { Random sequence generation (selection } \\
\text { bias) }\end{array}$ & High risk & $\begin{array}{l}\text { Alternating assignment stratified by re- } \\
\text { ferred or screened }\end{array}$ \\
\hline Allocation concealment (selection bias) & Unclear risk & Insufficient information available to assess \\
\hline $\begin{array}{l}\text { Incomplete outcome data (attrition bias) } \\
\text { All outcomes }\end{array}$ & Unclear risk & $\begin{array}{l}\text { Short-term loss to follow-up based on } \\
\text { primary outcome (composite measure of } \\
\text { high end state functioning -current MDD, } \\
\text { CESD, SF MCS) was: overall } 56 / 232 \\
(24 \%), 32 / 119(27 \%) \text { intervention and } 24 / \\
113(21 \%) \text { control. Reasons for loss to fol- } \\
\text { low-up provided, with similar reasons for } \\
\text { missing data across groups. Intention-to- } \\
\text { treat analysis reported, no description of } \\
\text { methods to manage missing data }\end{array}$ \\
\hline Selective reporting (reporting bias) & Low risk & $\begin{array}{l}\text { Protocol available and all prespecified out- } \\
\text { comes reported }\end{array}$ \\
\hline Other bias & Unclear risk & Insufficient information available to assess \\
\hline Implementation Integrity & Unclear risk & Insufficient information available to assess \\
\hline $\begin{array}{l}\text { Blinding of participants and personnel } \\
\text { (performance bias) } \\
\text { All outcomes }\end{array}$ & High risk & $\begin{array}{l}\text { Participants and personnel could not be } \\
\text { blinded, outcome likely to be influenced by } \\
\text { lack of blinding }\end{array}$ \\
\hline $\begin{array}{l}\text { Blinding of outcome assessment (detection } \\
\text { bias) } \\
\text { All outcomes }\end{array}$ & Low risk & $\begin{array}{l}\text { Assessor was not aware of treatment alloca- } \\
\text { tion }\end{array}$ \\
\hline
\end{tabular}

Roy-Byrne 2010

Methods

Participants
Study design: Randomised controlled trial

Setting: Primary care

Diagnosis: Meeting DSM-IV criteria for one or more of panic disorder, generalised anxiety disorder, social anxiety disorder, or post-traumatic stress disorder based on the Mini International Neuropsychiatric Interview and scoring at least 8 (moderate anxiety symptoms on a scale ranging from $0-20$ ) on the Overall Anxiety Severity and Impairment Scale (OASIS)

Inclusion criteria: $18-75$ years

Exclusion criteria: Persons unlikely to benefit from the Coordinated Anxiety Learning and Management (i.e. unstable medical conditions, marked cognitive impairment, active suicidal intent or plan, psychosis, bipolar I disorder, substance abuse of dependence except for alcohol and marijuana abuse), receiving ongoing CBT or medication from a psychiatrist, unable to speak English or Spanish

Age: Mean 43.5 (SD 13.4) years 
Gender: $71 \%$ female

Ethnicity: $57 \%$ white

Country: United States

Sample size (randomised): Total participants 1004, intervention 503, control 501

Interventions

Intervention: Stepped Co-ordinated Anxiety Learning and Management (CALM)

Contains the four elements of collaborative care:

1) a multi-professional approach to patient care: Primary care provider (PCP), social workers, nurses, psychologists (CM), psychologist/psychiatrist ( $\mathrm{MH}$ specialist)

2) a structured management plan: Patient choice of CBT, medication or both during 10-

12 weeks. The computerised CBT programme included 5 generic modules (education, self-monitoring, hierarchy development, breathing training, and relapse prevention) and 3 modules (cognitive restructuring and exposure to internal and external stimuli) tailored to each anxiety disorder and included psycho-educational materials and instructions for skills practice and exposure. CMs entered data which then created a personalised workbook and homework assignments. The medication algorithm emphasised selective serotonin reuptake inhibitors (SSRIs) or serotonin and norepinephrine reuptake inhibitors (SNRIs), dose optimisation, adverse effect monitoring, followed by second and third step combinations of $2 \mathrm{ADs}$ or an $\mathrm{AD}$ and benzodiazepine for non-response. CMs provided adherence monitoring, counselling to avoid alcohol and optimise sleep hygiene and behavioural activity. Non-responders could receive more of the same (stepping up) or the alternative modality (stepping over) for up to 3 more steps of treatment. After treatment completion, patients were entered into continued care and received monthly follow-up telephone calls to reinforce CBT skills, medication adherence, or both. If symptoms reemerged within the first 9 months patients were referred back a step

3) scheduled patient follow-ups: computerisedCBT $=6$ to 8 weekly sessions in 3 months, maintenance $=$ monthly follow up calls

4) enhanced inter-professional communication: CMs relayed medication suggestions from $\mathrm{MH}$ specialist to the PCP. CMs interacted regularly with the PCP both face-toface and via written communication. $\mathrm{MH}$ specialist provided PCPs with a medication algorithm and as needed consultation by telephone or email. CMs had weekly supervision with $\mathrm{MH}$ specialist plus cross-site monthly conference supervision calls

Control: Treatment as usual

Outcomes

Anxiety (BSI-12): 6, 12, 18 months

Medication use: 6, 12, 18 months

Quality of Life (mental and physical health): 6, 12, 18 months

Satisfaction: 6, 12, 18 months

Notes

CBT: cognitive behaviour therapy; CM: case manager; DSM-IV: Diagnostic and Statistical Manual fourth edition; $\mathrm{MH}$ : mental health; PCP: primary care provider

\section{Risk of bias}

Bias

Random sequence generation (selection Low risk bias)
Authors' judgement

k


Roy-Byrne 2010 (Continued)

\begin{tabular}{|c|c|c|}
\hline Allocation concealment (selection bias) & Low risk & $\begin{array}{l}\text { Block size was masked to all clinical site } \\
\text { study members }\end{array}$ \\
\hline $\begin{array}{l}\text { Incomplete outcome data (attrition bias) } \\
\text { All outcomes }\end{array}$ & Low risk & $\begin{array}{l}\text { Short-term loss to follow-up based on pri- } \\
\text { mary anxiety outcome (BSI-12 response) } \\
\text { was: overall } 128 / 1004 \text { (13\%), } 57 / 503 \\
\text { ( } 11 \%) \text { intervention and } 71 / 501 \text { (14\%) } \\
\text { control. Reasons for loss to follow-up pro- } \\
\text { vided, with similar reasons across groups. } \\
\text { Used intention-to-treat analysis }\end{array}$ \\
\hline Selective reporting (reporting bias) & Low risk & $\begin{array}{l}\text { Protocol available and all prespecified out- } \\
\text { comes reported }\end{array}$ \\
\hline Other bias & Unclear risk & Insufficient information available to assess \\
\hline Implementation Integrity & Low risk & $\begin{array}{l}\text { Attempts were made to assess implemen- } \\
\text { tation integrity (e.g. direct observation or } \\
\text { rating of tapes) }\end{array}$ \\
\hline $\begin{array}{l}\text { Blinding of participants and personnel } \\
\text { (performance bias) } \\
\text { All outcomes }\end{array}$ & High risk & $\begin{array}{l}\text { Participants and personnel could not be } \\
\text { blinded, outcome likely to be influenced by } \\
\text { lack of blinding }\end{array}$ \\
\hline $\begin{array}{l}\text { Blinding of outcome assessment (detection } \\
\text { bias) } \\
\text { All outcomes }\end{array}$ & Low risk & $\begin{array}{l}\text { Assessor was not aware of treatment alloca- } \\
\text { tion }\end{array}$ \\
\hline
\end{tabular}

Rubenstein 2002

\begin{tabular}{|c|c|}
\hline Methods & Study design: Cluster-randomised controlled trial \\
\hline Participants & $\begin{array}{l}\text { Setting: Primary care } \\
\text { Diagnosis: Major depression based on the Composite International Diagnostic Interview } \\
\text { (CIDI) } \\
\text { Inclusion criteria: Consecutive patients attending primary care appointments } \\
\text { Exclusion criteria: Not stated } \\
\text { Age: Mean } 47.7 \text { years } \\
\text { Gender: } 60 \% \text { female } \\
\text { Ethnicity: } 75 \% \text { white } \\
\text { Country: United States } \\
\text { Sample size (randomised): Total clusters 9, intervention 6, control 3; Total participants } \\
\text { 567, intervention 369, control } 198\end{array}$ \\
\hline Interventions & $\begin{array}{l}\text { Intervention: Evidence Based Quality Improvement depression care } \\
\text { Contains the four elements of collaborative care: } \\
\text { 1) a multi-professional approach to patient care: Primary care clinician (PCP), MH } \\
\text { nurse, psychologist, pharmacist (CM), psychiatrist ( } \mathrm{MH} \text { specialist) }\end{array}$ \\
\hline
\end{tabular}


Rubenstein 2002 (Continued)

2) a structured management plan: Each area were given guidance, training and materials and then left to implement collaborative care which included: provider education and decision support (training and feedback on performance), patient education (classes and written materials), screening/detection (CMs screen for depression, computer reminders, monitoring/enforcement), assessment (provider depression assessment worksheet, provider assessment reminders), care management, collaboration with $\mathrm{MH}$ specialists (improved referral process to $\mathrm{MH}$ speciality, $\mathrm{MH}$ specialist gives feedback to PCP)

3) scheduled patient follow-ups: Patient education $=8$ sessions

4) enhanced inter-professional communication: PCPs were sent computer reminders re monitoring of care and received feedback from $\mathrm{MH}$ specialists

Control: Treatment as usual enhanced as PCPs were mailed copies of clinical practice guidelines for depression

Outcomes

Depression (Poor depression response - MDD, CESD, SF, MCS): 6, 12 months

Quality of Life (mental and physical health): 12 months

Satisfaction: 6 months

Notes

CIDI: Composite International Diagnostic Interview; CM: case manager; MDD: major

depressive disorder; MH: mental health; PCP: primary care provider

\section{Risk of bias}

\begin{tabular}{|c|c|c|}
\hline Bias & Authors' judgement & Support for judgement \\
\hline $\begin{array}{l}\text { Random sequence generation (selection } \\
\text { bias) }\end{array}$ & Unclear risk & $\begin{array}{l}\text { Randomly assigned by an individual not } \\
\text { involved in patient recruitment }\end{array}$ \\
\hline Allocation concealment (selection bias) & Unclear risk & Insufficient information available to assess \\
\hline $\begin{array}{l}\text { Incomplete outcome data (attrition bias) } \\
\text { All outcomes }\end{array}$ & Unclear risk & $\begin{array}{l}\text { Short-term loss to follow-up based on pri- } \\
\text { mary depression outcome (poor depres- } \\
\text { sion outcomes) was: overall } 133 / 567(23 \%) \\
, 87 / 369(24 \%) \text { intervention and } 46 / 198 \\
(23 \%) \text { control. Reasons for loss to follow- } \\
\text { up not provided. Intention-to-treat analy- } \\
\text { sis not reported, no description of methods } \\
\text { to manage missing data }\end{array}$ \\
\hline Selective reporting (reporting bias) & Unclear risk & Insufficient information available to assess \\
\hline Other bias & Unclear risk & Insufficient information available to assess \\
\hline Implementation Integrity & Unclear risk & Insufficient information available to assess \\
\hline $\begin{array}{l}\text { Blinding of participants and personnel } \\
\text { (performance bias) } \\
\text { All outcomes }\end{array}$ & High risk & $\begin{array}{l}\text { Participants and personnel could not be } \\
\text { blinded, outcome likely to be influenced by } \\
\text { lack of blinding }\end{array}$ \\
\hline
\end{tabular}


Rubenstein 2002 (Continued)

Blinding of outcome assessment (detection Low risk bias)

All outcomes
Assessor was not aware of treatment allocation

Simon 2000a

\begin{tabular}{ll}
\hline Methods & Study design: Randomised controlled trial \\
\hline Participants & Setting: Primary care \\
& Diagnosis: Depression. Based on antidepressant prescription and also used a 20 item \\
depression scale from the Hopkins symptom checklist & Inclusion criteria: Patients at participating clinics who had received new prescriptions \\
& for antidepressants, with "new" defined as no antidepressant use in the previous 120 days \\
& Exclusion criteria: Not been diagnosed with depression at any visit (nondepression indi- \\
& cation for prescription); had been diagnosed with bipolar disorder or psychotic disorder \\
& in the previous two years; had been diagnosed with alcohol or other substance misuse in \\
the previous 90 days; or had visited a psychiatrist in the previous 90 days & Age: Mean 46.6 years \\
Gender: $73 \%$ female & Ethnicity: Not stated \\
Country: United States & Sample size (randomised): Total participants 392, intervention 196, control 196
\end{tabular}

Interventions

Intervention: Care management

Contains the four elements of collaborative care:

1) a multi-professional approach to patient care: Primary care provider (PCP), nurse (CM), psychiatrist ( $\mathrm{MH}$ specialist)

2) a structured management plan: CMs assessed current use of ADs, side effects, and severity of depression. CMs supported PCPs by communicating urgent recommendations, assisting with arranging follow up visits, telephoning patients who had discontinued treatment, and helping with referrals. Telephone contacts sometimes included general support and encouragement but did not include any specific psychotherapeutic content. CMs helped with medication management but were not expected to make prescribing decisions but did recommend dosage changes or changes to different $\mathrm{AD}$ 3) scheduled patient follow-ups: 3 telephone calls at beginning, 8 and 16 weeks 4) enhanced inter-professional communication: After each telephone assessment PCPs received a feedback report including computerised data, assessment data, and sophisticated algorithm based recommendations. CMs received weekly supervision from $\mathrm{MH}$ specialist

Control: Treatment as usual

\section{Risk of bias}


Simon 2000a (Continued)

\begin{tabular}{|c|c|c|}
\hline Bias & Authors' judgement & Support for judgement \\
\hline $\begin{array}{l}\text { Random sequence generation (selection } \\
\text { bias) }\end{array}$ & Low risk & Computer generated \\
\hline Allocation concealment (selection bias) & Unclear risk & Insufficient information available to assess \\
\hline $\begin{array}{l}\text { Incomplete outcome data (attrition bias) } \\
\text { All outcomes }\end{array}$ & Unclear risk & $\begin{array}{l}\text { Short-term loss to follow-up based on pri- } \\
\text { mary depression outcome (HSCL-20 50\% } \\
\text { decrease) was: overall 20/392 (5\%), } 10 / 196 \\
(5 \%) \text { intervention and 10/196 (5\%) con- } \\
\text { trol. Reasons for loss to follow-up not pro- } \\
\text { vided. Used intention-to-treat analysis }\end{array}$ \\
\hline Selective reporting (reporting bias) & Unclear risk & Insufficient information available to assess \\
\hline Other bias & Unclear risk & Insufficient information available to assess \\
\hline Implementation Integrity & Unclear risk & Insufficient information available to assess \\
\hline $\begin{array}{l}\text { Blinding of participants and personnel } \\
\text { (performance bias) } \\
\text { All outcomes }\end{array}$ & High risk & $\begin{array}{l}\text { Participants and personnel could not be } \\
\text { blinded, outcome likely to be influenced by } \\
\text { lack of blinding }\end{array}$ \\
\hline $\begin{array}{l}\text { Blinding of outcome assessment (detection } \\
\text { bias) } \\
\text { All outcomes }\end{array}$ & Low risk & $\begin{array}{l}\text { Assessor was not aware of treatment alloca- } \\
\text { tion }\end{array}$ \\
\hline
\end{tabular}

\section{Simon 2000b}

Methods

Participants
Study design: Randomised controlled trial

Setting: Primary care

Diagnosis: Depression. Based on antidepressant prescription and also used a 20 item depression scale from the Hopkins symptom checklist

Inclusion criteria: Patients at participating clinics who had received new prescriptions for antidepressants, with "new" defined as no antidepressant use in the previous 120 days Exclusion criteria: Not been diagnosed with depression at any visit (nondepression indication for prescription); had been diagnosed with bipolar disorder or psychotic disorder in the previous two years; had been diagnosed with alcohol or other substance misuse in the previous 90 days; or had visited a psychiatrist in the previous 90 days

Age: Mean 46.7 years

Gender: $71 \%$ female

Ethnicity: Not stated

Country: United States

Sample size (randomised): Total participants 417, intervention 196, control 221 
Simon 2000b (Continued)

\begin{tabular}{|c|c|c|}
\hline Interventions & \multicolumn{2}{|c|}{$\begin{array}{l}\text { Intervention: Care management } \\
\text { Contains the four elements of collaborative care: } \\
\text { 1) a multi-professional approach to patient care: Primary care provider (PCP), nurse } \\
\text { (CM), psychiatrist (MH specialist) } \\
\text { 2) a structured management plan: CMs assessed current use of ADs, side effects, and } \\
\text { severity of depression. CMs supported PCPs by communicating urgent recommenda- } \\
\text { tions, assisting with arranging follow up visits, telephoning patients who had discon- } \\
\text { tinued treatment, and helping with referrals. Telephone contacts sometimes included } \\
\text { general support and encouragement but did not include any specific psychotherapeutic } \\
\text { content. CMs helped with medication management but were not expected to make pre- } \\
\text { scribing decisions but did recommend dosage changes or changes to different AD } \\
\text { 3) scheduled patient follow-ups: } 3 \text { telephone calls at beginning, } 8 \text { and } 16 \text { weeks } \\
\text { 4) enhanced inter-professional communication: After each telephone assessment PCPs } \\
\text { received a feedback report including computerised data, assessment data, and sophisti- } \\
\text { cated algorithm based recommendations. CMs received weekly supervision from MH } \\
\text { specialist } \\
\text { Control: Feedback only. PCPs received a detailed report on each patient eight and } 16 \\
\text { weeks after the initial prescription. These included computerised data (AD dosage and } \\
\text { repeat prescriptions, number of follow up visits, and arranged visits) and treatment } \\
\text { recommendations on the basis of a computerised algorithm }\end{array}$} \\
\hline Outcomes & \multicolumn{2}{|c|}{$\begin{array}{l}\text { Depression (HSCL-20): 3, } 6 \text { months } \\
\text { Medication use: } 6 \text { months }\end{array}$} \\
\hline Notes & \multicolumn{2}{|c|}{$\begin{array}{l}\text { AD: antidepressant; CM: case manager; HSCL: Hopkins Symptom Checklist; MH: } \\
\text { mental health; PCP: primary care provider }\end{array}$} \\
\hline \multicolumn{3}{|l|}{ Risk of bias } \\
\hline Bias & Authors' judgement & Support for judgement \\
\hline $\begin{array}{l}\text { Random sequence generation (selection } \\
\text { bias) }\end{array}$ & Low risk & Computer generated \\
\hline Allocation concealment (selection bias) & Unclear risk & Insufficient information available to assess \\
\hline $\begin{array}{l}\text { Incomplete outcome data (attrition bias) } \\
\text { All outcomes }\end{array}$ & Unclear risk & $\begin{array}{l}\text { Short-term loss to follow-up based on pri- } \\
\text { mary depression outcome (HSCL-20 50\% } \\
\text { decrease) was: overall } 21 / 392(5 \%), 10 / 196 \\
(5 \%) \text { intervention and } 11 / 221(5 \%) \text { con- } \\
\text { trol. Reasons for loss to follow-up not pro- } \\
\text { vided. Used intention-to-treat analysis }\end{array}$ \\
\hline Selective reporting (reporting bias) & Unclear risk & Insufficient information available to assess \\
\hline Other bias & Unclear risk & Insufficient information available to assess \\
\hline Implementation Integrity & Unclear risk & Insufficient information available to assess \\
\hline
\end{tabular}

Collaborative care for depression and anxiety problems (Review)

Copyright (־ 2012 The Cochrane Collaboration. Published by John Wiley \& Sons, Ltd. 
Simon 2000b (Continued)

Blinding of participants and personnel High risk (performance bias)

All outcomes
Participants and personnel could not be blinded, outcome likely to be influenced by lack of blinding

Blinding of outcome assessment (detection Low risk bias)

All outcomes

Simon 2004a

\begin{tabular}{|c|c|}
\hline Methods & Study design: Randomised controlled trial \\
\hline Participants & $\begin{array}{l}\text { Setting: Primary care } \\
\text { Diagnosis: Depression assessed by Hopkins Symptom Checklist Depression Scale } \\
\text { (HSCL) and Patient Health Questionnaire (PHQ). Those already in remission at the } \\
\text { baseline assessment (i.e. HSCL depression score }<0.5 \text { ) were excluded } \\
\text { Inclusion criteria: Primary care patients beginning antidepressant treatment for depres- } \\
\text { sion } \\
\text { Exclusion criteria: Receiving psychotherapy, those already in remission when contacted, } \\
\text { diagnosis of bipolar or schizophrenia in the last } 2 \text { years, cognitive, language, or hearing } \\
\text { impairment severe enough to preclude participation } \\
\text { Age: Mean } 44.5 \text { years } \\
\text { Gender: } 75 \% \text { female } \\
\text { Ethnicity: } 80 \% \text { white } \\
\text { Country: United States } \\
\text { Sample size (randomised): Total participants } 402 \text {, intervention } 207 \text {, control } 195\end{array}$ \\
\hline
\end{tabular}

Intervention: Telephone care management

Contains the four elements of collaborative care:

1) a multi-professional approach to patient care: Primary care physician (PCP), $\mathrm{MH}$ clinician (CM), psychologist/psychiatrist (MH specialist)

2) a structured management plan: Using scripts and motivational enhancement techniques each CM telephone call included a brief, structured assessment of depressive symptoms, $\mathrm{AD}$ use, and adverse effects. CMs also provided crisis intervention and referral to mental health specialty care when necessary. Patients received a detailed selfmanagement workbook emphasising behavioural activation, identifying and challenging negative thoughts, and developing a long-term self-care plan. CMs recommended reading the workbook but did not provide any specific counselling

3) scheduled patient follow-ups: 3 telephone contacts (weeks 4, 8 and 16) and 2 written mailings (weeks 26 and 36)

4) enhanced inter-professional communication: CMs sent PCPs a structured report of each contact including a summary of clinical progress and computer-generated recommendations regarding medication adjustment. If a change in treatment was recommended, the CM contacted the PCP to facilitate patient-physician communication and follow-up. CMs received weekly supervision from $\mathrm{MH}$ specialists

Control: Treatment as usual 
Simon 2004a (Continued)

\begin{tabular}{ll}
\hline Outcomes & $\begin{array}{l}\text { Depression (HSCL): } 6 \text { weeks, 3, 6, 9, 12, 15, } 18 \text { months } \\
\text { Medication use: } 6 \text { months } \\
\text { Satisfaction: 3, } 6 \text { months }\end{array}$ \\
\hline Notes & $\begin{array}{l}\text { CM: case manager; HSCL: Hopkins Symptom Checklist; MH: mental health; PCP: } \\
\text { primary care provider }\end{array}$ \\
\hline
\end{tabular}

\section{Risk of bias}

\begin{tabular}{|c|c|c|}
\hline Bias & Authors' judgement & Support for judgement \\
\hline $\begin{array}{l}\text { Random sequence generation (selection } \\
\text { bias) }\end{array}$ & Low risk & Computer generated \\
\hline Allocation concealment (selection bias) & Low risk & $\begin{array}{l}\text { Allocation conducted centrally by an indi- } \\
\text { vidual not involved in patient recruitment }\end{array}$ \\
\hline $\begin{array}{l}\text { Incomplete outcome data (attrition bias) } \\
\text { All outcomes }\end{array}$ & Unclear risk & $\begin{array}{l}\text { Short-term loss to follow-up based on pri- } \\
\text { mary depression outcome (HSCL 50\% im- } \\
\text { provement) was: overall 42/402 (10\%), 23/ } \\
207(11 \%) \text { intervention and 19/195 (10\%) } \\
\text { control. Reasons for loss to follow-up not } \\
\text { provided. Used intention-to-treat analysis }\end{array}$ \\
\hline Selective reporting (reporting bias) & Unclear risk & Insufficient information available to assess \\
\hline Other bias & Unclear risk & Insufficient information available to assess \\
\hline Implementation Integrity & Low risk & $\begin{array}{l}\text { Attempts were made to assess implemen- } \\
\text { tation integrity (e.g. direct observation or } \\
\text { rating of tapes) }\end{array}$ \\
\hline $\begin{array}{l}\text { Blinding of participants and personnel } \\
\text { (performance bias) } \\
\text { All outcomes }\end{array}$ & High risk & $\begin{array}{l}\text { Participants and personnel could not be } \\
\text { blinded, outcome likely to be influenced by } \\
\text { lack of blinding }\end{array}$ \\
\hline $\begin{array}{l}\text { Blinding of outcome assessment (detection } \\
\text { bias) } \\
\text { All outcomes }\end{array}$ & Low risk & $\begin{array}{l}\text { Assessor was not aware of treatment alloca- } \\
\text { tion }\end{array}$ \\
\hline
\end{tabular}

\section{Simon 2004b}

\begin{tabular}{ll}
\hline Methods & Study design: Randomised controlled trial \\
\hline Participants & Setting: Primary care \\
& Diagnosis: Depression assessed by Hopkins Symptom Checklist Depression Scale \\
& (HSCL) and Patient Health Questionnaire (PHQ) Those already in remission at the \\
baseline assessment (i.e. HSCL depression score $<0.5)$ were excluded
\end{tabular}


Inclusion criteria: Primary care patients beginning antidepressant treatment for depression

Exclusion criteria: Receiving psychotherapy, those already in remission when contacted, diagnosis of bipolar or schizophrenia in the last 2 years, cognitive, language, or hearing impairment severe enough to preclude participation

Age: Mean 44.4 years

Gender: $76 \%$ female

Ethnicity: $77 \%$ white

Country: United States

Sample size (randomised): Total participants 393, intervention 198, control 195

Interventions

Outcomes

Notes
Intervention: Telephone care management plus telephone psychotherapy

Contains the four elements of collaborative care:

1) a multi-professional approach to patient care: Primary care physician (PCP), MH clinician (CM), psychologist/psychiatrist (MH specialist)

2) a structured management plan: Using scripts and motivational enhancement techniques each CM telephone call included a brief, structured assessment of depressive symptoms, $\mathrm{AD}$ use, and adverse effects. CMs also provided crisis intervention and referral to mental health specialty care when necessary. Patients received a detailed selfmanagement workbook emphasising behavioural activation, identifying and challenging negative thoughts, and developing a long-term self-care plan. CMs recommended reading the workbook but did not provide any specific counselling. CBT sessions lasted 30 to 40 minutes and included: session 1 involved a detailed assessment and motivational enhancement exercises; sessions $2-4$ focused on increasing pleasant and rewarding activities; sessions 5-7 focused on identifying, challenging, and distancing from negative thoughts; session 8 focused on creation of a personal self-care plan covering medication use, self-monitoring, and self-management skills

3) scheduled patient follow-ups: 3 telephone contacts (weeks 4, 8 and 16) and 2 written mailings (weeks 26 and 36) plus 8 session CBT with psychotherapist

4) enhanced inter-professional communication: CMs sent PCPs a structured report of each contact including a summary of clinical progress and computer-generated recommendations regarding medication adjustment. If a change in treatment was recommended, the CM contacted the PCP to facilitate patient-physician communication and follow-up. CMs received weekly supervision from $\mathrm{MH}$ specialists

Control: Treatment as usual

Depression (HSCL): 6 weeks, 3, 6, 9, 12, 15, 18 months

Medication use: $6,12,18$ months

Satisfaction: 3, 6 months

AD: antidepressant; CBT: cognitive behaviour therapy; CM: case manager; HSCL: Hopkins Symptom Checklist; MH: mental health; PCP: primary care provider

\section{Risk of bias}

Bias Authors' judgement

Random sequence generation (selection Low risk bias)

\section{Support for judgement}

Computer generated 


\section{Simon 2004b (Continued)}

\begin{tabular}{|c|c|c|}
\hline Allocation concealment (selection bias) & Low risk & $\begin{array}{l}\text { Allocation conducted centrally by an indi- } \\
\text { vidual not involved in patient recruitment }\end{array}$ \\
\hline $\begin{array}{l}\text { Incomplete outcome data (attrition bias) } \\
\text { All outcomes }\end{array}$ & Unclear risk & $\begin{array}{l}\text { Short-term loss to follow-up based on pri- } \\
\text { mary depression outcome (HSCL 50\% im- } \\
\text { provement) was: overall 45/393 (11\%), 26/ } \\
198(13 \%) \text { intervention and 19/195 (10\%) } \\
\text { control. Reasons for loss to follow-up not } \\
\text { provided. Used intention-to-treat analysis }\end{array}$ \\
\hline Selective reporting (reporting bias) & Unclear risk & Insufficient information available to assess \\
\hline Other bias & Unclear risk & Insufficient information available to assess \\
\hline Implementation Integrity & Low risk & $\begin{array}{l}\text { Attempts were made to assess implemen- } \\
\text { tation integrity (e.g. direct observation or } \\
\text { rating of tapes) }\end{array}$ \\
\hline $\begin{array}{l}\text { Blinding of participants and personnel } \\
\text { (performance bias) } \\
\text { All outcomes }\end{array}$ & High risk & $\begin{array}{l}\text { Participants and personnel could not be } \\
\text { blinded, outcome likely to be influenced by } \\
\text { lack of blinding }\end{array}$ \\
\hline $\begin{array}{l}\text { Blinding of outcome assessment (detection } \\
\text { bias) } \\
\text { All outcomes }\end{array}$ & Low risk & $\begin{array}{l}\text { Assessor was not aware of treatment alloca- } \\
\text { tion }\end{array}$ \\
\hline
\end{tabular}

\section{Simon 2011}

\begin{tabular}{|c|c|}
\hline Methods & Study design: Randomised controlled trial \\
\hline Participants & $\begin{array}{l}\text { Setting: Primary care } \\
\text { Diagnosis: } 20 \text {-item depression scale from the Hopkins Symptom Checklist (HSCL) was } \\
\text { used } \\
\text { Inclusion criteria: Age } 18 \text { or older, filled a new antidepressant prescription from a par- } \\
\text { ticipating primary care physician; did not fill any prescription for antidepressant medi- } \\
\text { cation in the prior } 270 \text { days; had a diagnosis of a depressive disorder associated with the } \\
\text { prescription; was registered to use online messaging } \\
\text { Exclusion criteria: Diagnosis of bipolar disorder or psychotic disorder or any prescription } \\
\text { for mood stabiliser or antipsychotic medication in the prior } 2 \text { years } \\
\text { Age: Mean } 45.5 \text { years } \\
\text { Gender: } 72 \% \text { female } \\
\text { Ethnicity: } 16 \% \text { ethnic minority } \\
\text { Country: United States } \\
\text { Sample size (randomised): Total participants } 208 \text {, intervention } 106 \text {, control } 102\end{array}$ \\
\hline Interventions & $\begin{array}{l}\text { Intervention: Depression care management programme } \\
\text { Contains the four elements of collaborative care: } \\
\text { 1) a multi-professional approach to patient care: Primary care provider (PCP), MH nurse }\end{array}$ \\
\hline
\end{tabular}


Simon 2011 (Continued)

\begin{tabular}{|c|c|c|}
\hline & \multicolumn{2}{|c|}{$\begin{array}{l}\text { 2) a structured management plan: CMs began each contact with a message containing } \\
\text { a link to an online assessment (depression questionnaire, and questions regarding use of } \\
\text { ADs, side effects, and reasons for discontinuation). An algorithm generated a suggested } \\
\text { response which CMs could tailor. CMs facilitated follow-up visits, supported changes } \\
\text { in medication, or facilitated referral for specialty care. Each contact included this cycle: } \\
\text { outreach message from CM, patient completion of online assessment, structured response } \\
\text { from CM, and follow-up communication with the patient and PCP as needed. Patients } \\
\text { were free to send additional messages or telephone the CM if needed. The CM was } \\
\text { expected to make outreach telephone calls in case of suicidal ideation or other urgent } \\
\text { clinical need } \\
\text { 3) scheduled patient follow-ups: } 4 \text { on-line messaging contacts (baseline and weeks } 2,6 \\
\text { and 10) } \\
\text { 4) enhanced inter-professional communication: CMs consulted with PCPs and commu- } \\
\text { nicated with PCPs using an electronic messaging system within the electronic medical } \\
\text { record. CMs had supervision with MH specialist (weekly for } 3 \text { months and monthly } \\
\text { thereafter) } \\
\text { Control: Treatment as usual }\end{array}$} \\
\hline Outcomes & \\
\hline Notes & \multicolumn{2}{|c|}{$\begin{array}{l}\text { CM: case manager; HSCL: Hopkins Symptom Checklist; MH: mental health; PCP: } \\
\text { primary care provider }\end{array}$} \\
\hline \multicolumn{3}{|l|}{ Risk of bias } \\
\hline Bias & Authors' judgement & Support for judgement \\
\hline $\begin{array}{l}\text { Random sequence generation (selection } \\
\text { bias) }\end{array}$ & Low risk & Computer generated \\
\hline Allocation concealment (selection bias) & Low risk & Computer generated \\
\hline $\begin{array}{l}\text { Incomplete outcome data (attrition bias) } \\
\text { All outcomes }\end{array}$ & Unclear risk & $\begin{array}{l}\text { Short-term loss to follow-up based on pri- } \\
\text { mary depression outcome (HSCL) was: } \\
\text { overall } 11 / 208(5 \%), 2 / 106(2 \%) \text { interven- } \\
\text { tion and } 9 / 102(9 \%) \text { control. Reasons for } \\
\text { loss to follow-up not provided. Used inten- } \\
\text { tion-to-treat analysis }\end{array}$ \\
\hline Selective reporting (reporting bias) & Unclear risk & Insufficient information available to assess \\
\hline Other bias & Unclear risk & Insufficient information available to assess \\
\hline Implementation Integrity & Unclear risk & Insufficient information available to assess \\
\hline
\end{tabular}

Collaborative care for depression and anxiety problems (Review) 


\section{Simon 2011 (Continued)}

Blinding of participants and personnel High risk (performance bias)

All outcomes
Participants and personnel could not be blinded, outcome likely to be influenced by lack of blinding

Blinding of outcome assessment (detection Low risk

bias)

Assessor was not aware of treatment alloca-

All outcomes

\section{Smit 2006a}

\begin{tabular}{ll}
\hline Methods & Study design: Randomised controlled trial \\
\hline Participants & Setting: Primary care \\
Diagnosis: Current Diagnostic and Statistical Manual (DSM-IV) diagnosis of major \\
depressive disorder, according to the primary care physician and confirmed by an inde- \\
pendent structured psychiatric interview (Composite International Diagnostic Interview \\
version 2.0$)$ \\
Inclusion criteria: Aged 18 - 70 years \\
Exclusion criteria: Patients suffering from a life threatening medical condition, a psy- \\
chotic disorder, dementia, alcohol addiction or drug abuse, women who were pregnant \\
or nursing, and patients already receiving mental health treatment elsewhere \\
Age: Mean 43.4 years \\
Gender: $65 \%$ female \\
Ethnicity: Not stated \\
Country: The Netherlands \\
Sample size (randomised: Depression Recurrance Programme): Total participants 184, \\
intervention 112 , control 72
\end{tabular}

Interventions

Intervention: Depression recurrence programme (DRP)

Contains the four elements of collaborative care:

1) a multi-professional approach to patient care: Primary care physician (PCP), $\mathrm{MH}$ nurse (CM), psychiatrist ( $\mathrm{MH}$ specialist)

2) a structured management plan: Depression recurrence programme consisted of structured/standardised psychoeducational sessions including medication management, selfcare, relapse prevention and support. Patients received a book and videotape about depression, treatment options, relapse prevention and self-management strategies. Face-toface contact provided overview of programme and collaboration between CM and PCP, personal and medication history, stress reduction techniques, preparation of a recurrence prevention plan, encouraging socialising and scheduling pleasant activities. Telephone contacts included symptom monitoring and changes in recurrence prevention plan and/ or medication

3) scheduled patient follow-ups: 3 face-to-face (2-4 weekly) and 4 telephone contacts (2.5 months after last face-to-face session then every 3 months for 3 years)

4) enhanced inter-professional communication: CMs sent written feedback to PCP after each face-to-face contact and as needed. CMs also sent PCPs a copy of the recurrence prevention plan and accompanying letter. CMs received regular supervision from $\mathrm{MH}$ specialist

Control: Treatment as usual 


\section{Smit 2006a (Continued)}

\begin{tabular}{ll}
\hline Outcomes & $\begin{array}{l}\text { Depression (DSM-IV recovered): 27 weeks } \\
\text { Medication use: 3, 6, 9, 12, 36 months } \\
\text { Quality of Life (mental and physical health): } 36 \text { months } \\
\text { Satisfaction: } 3 \text { months }\end{array}$ \\
\hline Notes & CM: case manager; MH: mental health; PCP: primary care provider \\
\hline
\end{tabular}

\section{Risk of bias}

\begin{tabular}{|c|c|c|}
\hline Bias & Authors' judgement & Support for judgement \\
\hline $\begin{array}{l}\text { Random sequence generation (selection } \\
\text { bias) }\end{array}$ & Low risk & Computer generated \\
\hline Allocation concealment (selection bias) & Low risk & $\begin{array}{l}\text { Centrally by telephone by an individual } \\
\text { not involved in patient recruitment, who } \\
\text { opened a sealed opaque envelope }\end{array}$ \\
\hline $\begin{array}{l}\text { Incomplete outcome data (attrition bias) } \\
\text { All outcomes }\end{array}$ & Low risk & $\begin{array}{l}\text { Short-term loss to follow-up based on } \\
\text { primary depression outcome (Recovered } \\
\text { DSM-IV) was: overall } 26 / 184(14 \%), 16 / \\
112(14 \%) \text { intervention and } 10 / 72(14 \%) \\
\text { control. Reasons for loss to follow-up pro- } \\
\text { vided, with similar reasons across groups. } \\
\text { Used intention-to-treat analysis }\end{array}$ \\
\hline Selective reporting (reporting bias) & Unclear risk & Insufficient information available to assess \\
\hline Other bias & Unclear risk & Insufficient information available to assess \\
\hline Implementation Integrity & Unclear risk & Insufficient information available to assess \\
\hline $\begin{array}{l}\text { Blinding of participants and personnel } \\
\text { (performance bias) } \\
\text { All outcomes }\end{array}$ & High risk & $\begin{array}{l}\text { Participants and personnel could not be } \\
\text { blinded, outcome likely to be influenced by } \\
\text { lack of blinding }\end{array}$ \\
\hline $\begin{array}{l}\text { Blinding of outcome assessment (detection } \\
\text { bias) } \\
\text { All outcomes }\end{array}$ & High risk & $\begin{array}{l}\text { Assessor was potentially aware of treatment } \\
\text { allocation }\end{array}$ \\
\hline
\end{tabular}

Smit 2006b

\begin{tabular}{ll}
\hline Methods & Study design: Randomised controlled trial \\
\hline Participants & $\begin{array}{l}\text { Setting: Primary Care } \\
\text { Diagnosis: Current Diagnostic and Statistical Manual (DSM-IV) diagnosis of major } \\
\text { depressive disorder, according to the primary care physician and confirmed by an inde- } \\
\text { pendent structured psychiatric interview (Composite International Diagnostic Interview }\end{array}$
\end{tabular}


Smit 2006b (Continued)

version 2.0)

Inclusion criteria: Aged 18 - 70 years

Exclusion criteria: Patients suffering from a life threatening medical condition, a psychotic disorder, dementia, alcohol addiction or drug abuse, women who were pregnant or nursing, and patients already receiving mental health treatment elsewhere

Age: Mean 42.6 years

Gender: $67 \%$ female

Ethnicity: Not stated

Country: The Netherlands

Sample size (randomised: Depression recurrence programme plus psychiatric consultation: Total participants 111, intervention 39, control 72

Interventions

Intervention: Depression recurrence programme (DRP) plus psychiatric consultation Contains the four elements of collaborative care:

1) a multi-professional approach to patient care: Primary care physician (PCP), $\mathrm{MH}$ nurse $(\mathrm{CM})$, psychiatrist ( $\mathrm{MH}$ specialist)

2) a structured management plan: DRP consisted of structured/standardised psychoeducational sessions including medication management, self-care, relapse prevention and support. Patients received a book and videotape about depression, treatment options, relapse prevention and self-management strategies. Face-to-face contact provided overview of programme and collaboration between CM and PCP, personal and medication history, stress reduction techniques, preparation of a recurrence prevention plan, encouraging socialising and scheduling pleasant activities. Telephone contacts included symptom monitoring and changes in recurrence prevention plan and/or medication. Prior to DRP a one 1-hour consultation with a psychiatrist was also offered who provided a report to PCP and CM

3) scheduled patient follow-ups: 3 face-to-face (2-4 weekly) and 4 telephone contacts (2.5 months after last face-to-face session then every 3 months for 3 years)

4) enhanced inter-professional communication: $\mathrm{MH}$ specialist sent PCP and CM report following patient consultation. CMs sent written feedback to PCP after each face-toface contact and as needed. CMs also sent PCPs a copy of the recurrence prevention plan and accompanying letter. $\mathrm{CMs}$ received regular supervision from $\mathrm{MH}$ specialist Control: Treatment as usual

Outcomes

Depression (DSM-IV recovered): 27 weeks

Medication use: 3, 6, 9, 12, 36 months

Quality of Life (mental and physical health): 36 months

Satisfaction: 3 months

Notes

Risk of bias

\begin{tabular}{|c|c|c|}
\hline Bias & Authors' judgement & Support for judgement \\
\hline
\end{tabular}

Random sequence generation (selection Low risk

Computer generated bias) 
Smit 2006b (Continued)

\begin{tabular}{|c|c|c|}
\hline Allocation concealment (selection bias) & Low risk & $\begin{array}{l}\text { Centrally by telephone by an individual } \\
\text { not involved in patient recruitment, who } \\
\text { opened a sealed opaque envelope }\end{array}$ \\
\hline $\begin{array}{l}\text { Incomplete outcome data (attrition bias) } \\
\text { All outcomes }\end{array}$ & Low risk & $\begin{array}{l}\text { Short-term loss to follow-up based on } \\
\text { primary depression outcome (Recovered } \\
\text { DSM-IV) was: overall } 17 / 111(15 \%), 7 / \\
39(18 \%) \text { intervention and } 10 / 72(14 \%) \\
\text { control. Reasons for loss to follow-up pro- } \\
\text { vided, with similar reasons across groups. } \\
\text { Used intention-to-treat analysis }\end{array}$ \\
\hline Selective reporting (reporting bias) & Unclear risk & Insufficient information available to assess \\
\hline Other bias & Unclear risk & Insufficient information available to assess \\
\hline Implementation Integrity & Unclear risk & Insufficient information available to assess \\
\hline $\begin{array}{l}\text { Blinding of participants and personnel } \\
\text { (performance bias) } \\
\text { All outcomes }\end{array}$ & High risk & $\begin{array}{l}\text { Participants and personnel could not be } \\
\text { blinded, outcome likely to be influenced by } \\
\text { lack of blinding }\end{array}$ \\
\hline $\begin{array}{l}\text { Blinding of outcome assessment (detection } \\
\text { bias) } \\
\text { All outcomes }\end{array}$ & High risk & $\begin{array}{l}\text { Assessor was potentially aware of treatment } \\
\text { allocation }\end{array}$ \\
\hline
\end{tabular}

\section{Smit 2006c}

\begin{tabular}{ll} 
Methods & Study design: Randomised controlled trial \\
\hline Participants & Setting: Primary care \\
& Diagnosis: Current Diagnostic and Statistical Manual (DSM-IV) diagnosis of major \\
depressive disorder, according to the primary care physician and confirmed by an inde- & pendent structured psychiatric interview (Composite International Diagnostic Interview \\
& version 2.0$)$ \\
& Inclusion criteria: Aged 18 - 70 years \\
& Exclusion criteria: Patients suffering from a life threatening medical condition, a psy- \\
chotic disorder, dementia, alcohol addiction or drug abuse, women who were pregnant & or nursing, and patients already receiving mental health treatment elsewhere \\
Age: Mean 43.5 years & Gender: $59.5 \%$ female \\
Ethnicity: Not stated \\
Country: The Netherlands \\
Sample size (randomised: Depression recurrence programme plus CBT): Total partici- \\
pants 116 , intervention 44, control 72
\end{tabular}


Smit 2006c (Continued)

\begin{tabular}{|c|c|}
\hline Interventions & $\begin{array}{l}\text { Intervention: Depression recurrence programme (DRP) plus CBT } \\
\text { Contains the four elements of collaborative care: } \\
\text { 1) a multi-professional approach to patient care: Primary care physician (PCP), MH } \\
\text { nurse (CM), psychiatrist (MH specialist) } \\
\text { 2) a structured management plan: Depression recurrence programme consisted of struc- } \\
\text { tured/standardised psychoeducational sessions including medication management, self- } \\
\text { care, relapse prevention and support. Patients received a book and videotape about de- } \\
\text { pression, treatment options, relapse prevention and self-management strategies. Face-to- } \\
\text { face contact provided overview of programme and collaboration between CM and PCP, } \\
\text { personal and medication history, stress reduction techniques, preparation of a recurrence } \\
\text { prevention plan, encouraging socialising and scheduling pleasant activities. Telephone } \\
\text { contacts included symptom monitoring and changes in recurrence prevention plan and/ } \\
\text { or medication. CBT provided after DRP involved 1-hour sessions with a CBT therapist } \\
\text { 3) scheduled patient follow-ups: } 3 \text { face-to-face ( } 2 \text { - } 4 \text { weekly) and } 4 \text { telephone contacts } \\
\text { (2.5 months after last face-to-face session then every } 3 \text { months for } 3 \text { years) plus } 10-12 \\
\text { face-to-face CBT sessions (10-12 weekly) } \\
\text { 4) enhanced inter-professional communication: CMs sent written feedback to PCP after } \\
\text { each face-to-face contact and as needed. CMs also sent PCPs a copy of the recurrence } \\
\text { prevention plan and accompanying letter. CMs received regular supervision from MH } \\
\text { specialist. At end of CBT the therapist informed CM of content and progress made } \\
\text { Control: Treatment as usual }\end{array}$ \\
\hline Outcomes & $\begin{array}{l}\text { Depression (DSM-IV recovered): } 27 \text { weeks } \\
\text { Medication use: } 3,6,9,12,36 \text { months } \\
\text { Quality of Life (mental and physical health): } 36 \text { months } \\
\text { Satisfaction: } 3 \text { months }\end{array}$ \\
\hline Notes & $\begin{array}{l}\text { CBT: cognitive behaviour therapy; CM: case manager; } \mathrm{MH} \text { : mental health; PCP: primary } \\
\text { care provider }\end{array}$ \\
\hline
\end{tabular}

Risk of bias

Bias Authors' judgement

Support for judgement

Random sequence generation (selection Low risk Computer generated bias)

Allocation concealment (selection bias) Low risk

Centrally by telephone by an individual not involved in patient recruitment, who opened a sealed opaque envelope

Incomplete outcome data (attrition bias) Low risk All outcomes

Short-term loss to follow-up based on primary depression outcome (Recovered DSM-IV) was: overall 18/116 (16\%), 8/ $44(18 \%)$ intervention and 10/72 (14\%) control. Reasons for loss to follow-up provided, with similar reasons across groups. Used intention-to-treat analysis 


\section{Smit 2006c (Continued)}

\begin{tabular}{|c|c|c|}
\hline Selective reporting (reporting bias) & Unclear risk & Insufficient information available to assess \\
\hline Other bias & Unclear risk & Insufficient information available to assess \\
\hline Implementation Integrity & Unclear risk & Insufficient information available to assess \\
\hline $\begin{array}{l}\text { Blinding of participants and personnel } \\
\text { (performance bias) } \\
\text { All outcomes }\end{array}$ & High risk & $\begin{array}{l}\text { Participants and personnel could not be } \\
\text { blinded, outcome likely to be influenced by } \\
\text { lack of blinding }\end{array}$ \\
\hline $\begin{array}{l}\text { Blinding of outcome assessment (detection } \\
\text { bias) } \\
\text { All outcomes }\end{array}$ & High risk & $\begin{array}{l}\text { Assessor was potentially aware of treatment } \\
\text { allocation }\end{array}$ \\
\hline
\end{tabular}

Strong 2008

Methods

Participants
Study design: Randomised controlled trial

Setting: Specialist, primary care

Diagnosis: Major depressive disorder. Patients with a score of 15 or more on the Hospital Anxiety and Depression Scale interviewed using the major depression section of the Structured Clinical Interview for the Diagnostic and Statistical Manual of Mental Disorders (DSM-IV). A minimum severity of major depressive disorder, defined by a score on the Symptom Checklist-20 (HSCL-20) depression scale of at least 1.75 (higher than the 1.5 which is usually regarded as equivalent to major depressive disorder, to allow for physical symptoms of cancer)

Inclusion criteria: A cancer prognosis of at least 6 months (to ensure that they could complete the trial); major depressive disorder of at least a month's duration that was not associated with major changes in the patient's cancer or its management (to ensure that we did not include patients with transient adjustment disorders)

Exclusion criteria: Patients who were unlikely to be able to adhere to the intervention: reasons included major communication difficulties such as severe deafness or dementia, inability to attend the cancer centre, concurrent intensive anticancer treatment such as frequent chemotherapy or radiotherapy, or another poorly controlled medical disorder such as epilepsy that dominated their care. We also excluded those who were receiving, or were judged to need, specialist psychiatric care (e.g., chronic major depressive disorder of more than 2 years' duration, severe substance or alcohol misuse, co morbid severe psychiatric disorder such as psychosis, or risk of suicide)

Age: Mean 56.6 years

Gender: $71 \%$ female

Ethnicity: Not stated

Country: United Kingdom

Sample size (randomised): Total participants 200, intervention 101, control 99

Interventions

Intervention: Depression Care for People with Cancer

Contains the four elements of collaborative care:

1) a multi-professional approach to patient care: General practitioner (PCP), nurse (CM) , psychiatrist (MH specialist) 
2) a structured management plan: Using a detailed manual CMs delivered patient education about depression and its treatment, problem-solving treatment to teach coping strategies and communication with each patient's oncologist and PCP. If patients decided to start or change $\mathrm{AD}$ the CMs encouraged them to contact PCP and then forwarded details to the PCP

3) scheduled patient follow-ups: 10 in three months (mostly face-to-face) then 3 telephone calls (monthly)

4) enhanced inter-professional communication: CMs communicated with PCPs and oncologists via phone or fax in relation to patient information or recommendations from $\mathrm{MH}$ specialist. CMs received weekly supervision from $\mathrm{MH}$ specialist

Control: Treatment as usual enhanced as PCPs and oncologists were informed of diagnosis and were given advice on choice of $\mathrm{AD}$ on request

\begin{tabular}{ll} 
Outcomes & $\begin{array}{l}\text { Depression (HSCL-20): 3, 6, } 12 \text { months } \\
\text { Medication use: 3, 6 months } \\
\text { Quality of Life (mental and physical health): 3, 6, } 12 \text { months }\end{array}$ \\
\hline Notes & AD: antidepressant; CM: case manager; HSCL: Hopkins Symptom Checklist; MH: \\
mental health; PCP: primary care provider
\end{tabular}

\section{Risk of bias}

\section{Bias}

Random sequence generation (selection Low risk bias)

\begin{tabular}{|c|c|c|}
\hline Allocation concealment (selection bias) & Low risk & Independent central service \\
\hline $\begin{array}{l}\text { Incomplete outcome data (attrition bias) } \\
\text { All outcomes }\end{array}$ & Low risk & $\begin{array}{l}\text { Short-term loss to follow-up based on pri- } \\
\text { mary depression outcome (HSCL-20 } \geq \\
50 \% \text { decrease) was: overall } 4 / 200(2 \%), 4 / \\
101(4 \%) \text { intervention and } 0 / 99(0 \%) \text { con- } \\
\text { trol. Used intention-to-treat analysis }\end{array}$ \\
\hline Selective reporting (reporting bias) & Unclear risk & Insufficient information available to assess \\
\hline Other bias & Unclear risk & Insufficient information available to assess \\
\hline Implementation Integrity & Low risk & $\begin{array}{l}\text { Attempts were made to assess implemen- } \\
\text { tation integrity (e.g. direct observation or } \\
\text { rating of tapes) }\end{array}$ \\
\hline $\begin{array}{l}\text { Blinding of participants and personnel } \\
\text { (performance bias) } \\
\text { All outcomes }\end{array}$ & High risk & $\begin{array}{l}\text { Participants and personnel could not be } \\
\text { blinded, outcome likely to be influenced by } \\
\text { lack of blinding }\end{array}$ \\
\hline
\end{tabular}

\section{Support for judgement}

Computer generated of blinding 
Strong 2008 (Continued)

Blinding of outcome assessment (detection Low risk

Assessor was not aware of treatment allocabias)

tion

All outcomes

Swindle 2003

Methods

Participants
Study design: Cluster-randomised controlled trial

Setting: Primary care

Diagnosis: Major depression, dysthymia, or partially remitted major depression using PRIME-MD structured diagnostic interview

Inclusion criteria: 2 or more general medicine clinic visits during the past year and plans to receive ongoing medical care from insurance group, access to a telephone and provided informed consent

Exclusion criteria: Incompetent for interview (e.g. active psychosis, dementia), residents of a nursing home, actively suicidal, seen in a Veterans Affairs Medical Centres mental health programme (made a visit during the previous 30 days and had a future appointment scheduled), active cocaine or opiate abuser, history of bipolar disorder, terminally ill (death expected within 12 months)

Age: Mean 56.3 years

Gender: 3\% female

Ethnicity: 86\% Caucasian

Country: United States

Sample size (randomised): Total clusters 2, intervention 1, control 1; Total participants 268 , intervention 134 , control 134
Intervention: Integrated care

Contains the four elements of collaborative care:

1) a multi-professional approach to patient care: Primary care physician (PCP), $\mathrm{MH}$ nurse specialist $(\mathrm{CM})$, psychiatrist (MH specialist)

2) a structured management plan: CMs and patients developed individual treatment plans including medication prescription (recommendation of initial 8 week course typically SSRI for those not currently on ADs), change in AD or dose (for those still symptomatic on current medication), onward referral to CBT or specialist care (for those with non-response to current medication); and liaison with PCP. CMs monitored progress including depressive symptoms, review side effects, encourage treatment compliance, schedule further visits to themselves or PCPs to modify medication and/or refer to mental health specialists

3) scheduled patient follow-ups: 4 contacts at baseline, 2 weeks, one month and two months (face-to-face or telephone)

4) enhanced inter-professional communication: CMs communicated the treatment plan to PCPs who discussed and amended as appropriate and discussions between CM and PCP preceded any further modifications. CM records were maintained in medical record. $\mathrm{CMs}$ attended monthly meetings and $\mathrm{MH}$ specialists were available for $\mathrm{CMs}$ when required

Control: Treatment as usual enhanced as PCPs received training in current treatment strategies for depression and how to use brief diagnostic interview. Patient's diagnosis was placed in medical record 
Swindle 2003 (Continued)

\begin{tabular}{ll}
\hline Outcomes & $\begin{array}{l}\text { Depression (BDI): 3, } 12 \text { months } \\
\text { Medication use: } 12 \text { months } \\
\text { Satisfaction: 3, } 12 \text { months }\end{array}$ \\
\hline Notes & $\begin{array}{l}\text { AD: antidepressant; BDI: Beck Depression Inventory; CM: case manager; MH: mental } \\
\text { health; PCP: primary care provider }\end{array}$ \\
\hline
\end{tabular}

Risk of bias

\begin{tabular}{|c|c|c|}
\hline Bias & Authors' judgement & Support for judgement \\
\hline $\begin{array}{l}\text { Random sequence generation (selection } \\
\text { bias) }\end{array}$ & Low risk & Coin-toss \\
\hline Allocation concealment (selection bias) & Unclear risk & Insufficient information available to assess \\
\hline $\begin{array}{l}\text { Incomplete outcome data (attrition bias) } \\
\text { All outcomes }\end{array}$ & Unclear risk & $\begin{array}{l}\text { Short-term loss to follow-up based on pri- } \\
\text { mary depression outcome (BDI) was: over- } \\
\text { all } 22 / 268(8 \%), 9 / 134(7 \%) \text { intervention } \\
\text { and } 13 / 134(10 \%) \text { control. Reasons for loss } \\
\text { to follow-up not provided by group. Used } \\
\text { intention-to-treat analysis }\end{array}$ \\
\hline Selective reporting (reporting bias) & Unclear risk & Insufficient information available to assess \\
\hline Other bias & Unclear risk & Insufficient information available to assess \\
\hline Implementation Integrity & Unclear risk & Insufficient information available to assess \\
\hline $\begin{array}{l}\text { Blinding of participants and personnel } \\
\text { (performance bias) } \\
\text { All outcomes }\end{array}$ & High risk & $\begin{array}{l}\text { Participants and personnel could not be } \\
\text { blinded, outcome likely to be influenced by } \\
\text { lack of blinding }\end{array}$ \\
\hline $\begin{array}{l}\text { Blinding of outcome assessment (detection } \\
\text { bias) } \\
\text { All outcomes }\end{array}$ & Low risk & $\begin{array}{l}\text { Assessor was not aware of treatment alloca- } \\
\text { tion }\end{array}$ \\
\hline
\end{tabular}

\section{Uebelacker 2011}

\begin{tabular}{|c|c|}
\hline Methods & Study design: Pilot randomised controlled trial \\
\hline Participants & $\begin{array}{l}\text { Setting: Primary care } \\
\text { Diagnosis: Meeting Diagnostic and Statistical Manual (DSM-IV) criteria for major de- } \\
\text { pression, minor depression, or dysthymia in the past year, or current elevated depressive } \\
\text { symptoms (Quick Inventory of Depression Symptoms [QIDS] score). Assessed using } \\
\text { Structured Clinical Interview for DSM-IV (SCID) and QIDS to determine whether the } \\
\text { participant met all eligibility criteria. If they did, the research staff administered the Cen- } \\
\text { tre for Epidemiological Studies Depression (CES-D) and World Health Organization }\end{array}$ \\
\hline
\end{tabular}


Uebelacker 2011 (Continued)

Disability Assessment Schedule II (WHO-DAS)

Inclusion criteria: Membership in the Medicaid Health Maintenance Organisation; selfidentified as Latino; having recently filled a prescription for an antidepressant medication for depression, prescribed by a primary care provider; not currently receiving services from a behavioural health specialist

Exclusion criteria: Not stated

Age: Mean 39.1 years

Gender: $95 \%$ female

Ethnicity: $100 \%$ Latino

Country: United States

Sample size (randomised): Total participants 38, intervention 19, control 19

Interventions

Intervention: Telephone depression care management (Depression Health Enhancement for Latino Patients: D-HELP)

Contains the four elements of collaborative care:

1) a multi-professional approach to patient care: Primary care physician (PCP), graduates (CM), social worker/psychiatrist (MH specialist)

2) a structured management plan: CMs supported treatment provided by PCPs by use of telephone calls consisting of a) assessment of depression symptoms, b) assessment of medication use/adherence, c) discussion of next follow-up appointment with PCP, and d) setting of depression treatment goals. DCMs assessed barriers toward meeting depression treatment goals and assisted with problem-solving to decrease barriers

3) scheduled patient follow-ups: 8 telephone calls (weekly for 4 weeks then fortnightly for 8 weeks)

4) enhanced inter-professional communication: CMs provided written feedback to PCPs for a minimum of once per month and more often if required. CMs received weekly supervision from $\mathrm{MH}$ specialist

Control: Treatment as usual

Outcomes

Depression (QIDS): 6, 12 weeks

Medication use: 3, 6 months

Quality of Life (mental and physical health): 6, 12 weeks

Satisfaction: 6, 12 weeks

Notes

CM: case manager; MH: mental health; PCP: primary care provider

Risk of bias

\begin{tabular}{|c|c|c|}
\hline Bias & Authors' judgement & Support for judgement \\
\hline $\begin{array}{l}\text { Random sequence generation (selection } \\
\text { bias) }\end{array}$ & Unclear risk & Insufficient information available to assess \\
\hline Allocation concealment (selection bias) & Unclear risk & Insufficient information available to assess \\
\hline $\begin{array}{l}\text { Incomplete outcome data (attrition bias) } \\
\text { All outcomes }\end{array}$ & High risk & $\begin{array}{l}\text { Short-term loss to follow-up based on pri- } \\
\text { mary depression outcome (QIDS) was: } \\
\text { overall } 15 / 38(39 \%), 7 / 19(37 \%) \text { interven- } \\
\text { tion and } 8 / 19(42 \%) \text { control. Reasons for }\end{array}$ \\
\hline
\end{tabular}

Collaborative care for depression and anxiety problems (Review)

Copyright $\odot 2012$ The Cochrane Collaboration. Published by John Wiley \& Sons, Ltd. 
Uebelacker 2011 (Continued)

loss to follow-up not provided. Intentionto-treat analysis not reported, no description of methods to manage missing data

\begin{tabular}{l|ll}
\hline Selective reporting (reporting bias) & Unclear risk & Insufficient information available to assess \\
\hline Other bias & Unclear risk & Insufficient information available to assess \\
\hline Implementation Integrity & Unclear risk & Insufficient information available to assess \\
\hline $\begin{array}{l}\text { Blinding of participants and personnel } \\
\text { (performance bias) } \\
\text { All outcomes }\end{array}$ & High risk & $\begin{array}{l}\text { Participants and personnel could not be } \\
\text { blinded, outcome likely to be influenced by } \\
\text { lack of blinding }\end{array}$
\end{tabular}

Blinding of outcome assessment (detection Low risk bias)

Assessor was not aware of treatment allocation

All outcomes

Unutzer 2002

Methods

Participants
Study design: Randomised controlled trial

Setting: Primary care

Diagnosis: Current major depression or dysthymic disorder according to the Structured Clinical Interview for DSM (SCID)

Inclusion criteria: Age 60 years or older, plans to use one of the participating clinics as the main source of general medical care in the coming year, English speaking Exclusion criteria: Current drinking problems, history of bipolar disorder or Psychosis, ongoing treatment with a psychiatrist, severe cognitive impairment, acute risk for suicide and needing immediate care, lack of transportation or access to a telephone Age: Mean 71.2 (SD 7.5) years

Gender: $65 \%$ female

Ethnicity: 23\% ethnic minority

Country: United States

Sample size (randomised): Total participants 1801, intervention 906, control 895

Interventions

Intervention: Improving Mood-Promoting Access to Collaborative Treatment (IMPACT)

Contains the four elements of collaborative care:

1) a multi-professional approach to patient care: Primary care physician (PCP), nurse/ psychologist (CM), academic PCP/psychiatrist (MH specialist)

2) a structured management plan: $\mathrm{CM}$ educates using a brochure and a videotape attempting to form a therapeutic alliance and encouraging patient to become an active participant in depression care. CMs discuss treatment preferences (including ADs and psychotherapy), offers follow-up for 1-year period and coordinates depression care with patient's PCP. CMs track clinical progress and monitor treatment side-effects at each contact or delivers problem solving therapy (PST). A 3-step treatment algorithm was developed allowing treatment team to establish a treatment plan for each patient's need 
Unutzer 2002 (Continued)

over time. Step 1: Start AD or PST. Non-response becomes step 2: alternative AD or from AD to PST, or vice versa. Non-response at step 2 is discussed and considered for psychiatric consultation and step 3: combination of treatments. Relapse prevention plans were developed when recovery achieved

3) scheduled patient follow-ups: PST=6-8 sessions. Acute phase CM contact=weekly or biweekly (telephone or face-to-face). Once symptoms in remission, follow-up about once per month

4) enhanced inter-professional communication: $\mathrm{CM}$ coordinates depression care with patient's PCP and receives weekly supervision from $\mathrm{MH}$ specialist who also sees patients if necessary

Control: Treatment as usual enhanced as patients were informed of their diagnosis and encouraged to follow-up with PCP

Outcomes

Depression (HSCL-20): 3, 6, 12, 18, 24 months

Medication use: 3, 6, 12, 18, 24 months

Quality of Life (mental and physical health): 3, 6, 12, 18, 24 months

Satisfaction: 3, 6, 12, 18, 24 months

Notes

AD: antidepressant; CM: case manager; HSCL: Hopkins Symptom Checklist; MH: mental health; PCP: primary care provider

Risk of bias

\begin{tabular}{|c|c|c|}
\hline Bias & Authors' judgement & Support for judgement \\
\hline $\begin{array}{l}\text { Random sequence generation (selection } \\
\text { bias) }\end{array}$ & Low risk & Computer generated \\
\hline Allocation concealment (selection bias) & Low risk & $\begin{array}{l}\text { Centrally prepared numbered sealed en- } \\
\text { velopes, used sequentially }\end{array}$ \\
\hline $\begin{array}{l}\text { Incomplete outcome data (attrition bias) } \\
\text { All outcomes }\end{array}$ & Low risk & $\begin{array}{l}\text { Short-term loss to follow-up based on pri- } \\
\text { mary depression outcome (HSCL-20 de- } \\
\text { pression) was: overall 231/1801 (13\%), } \\
105 / 906(12 \%) \text { intervention and } 126 / 895 \\
\text { (14\%) control. Reasons for loss to follow- } \\
\text { up provided, with similar reasons across } \\
\text { groups. Used intention-to-treat analysis }\end{array}$ \\
\hline Selective reporting (reporting bias) & Low risk & $\begin{array}{l}\text { Protocol available and all prespecified out- } \\
\text { comes reported }\end{array}$ \\
\hline Other bias & Unclear risk & Insufficient information available to assess \\
\hline Implementation Integrity & Unclear risk & Insufficient information available to assess \\
\hline $\begin{array}{l}\text { Blinding of participants and personnel } \\
\text { (performance bias) } \\
\text { All outcomes }\end{array}$ & High risk & $\begin{array}{l}\text { Participants and personnel could not be } \\
\text { blinded, outcome likely to be influenced by } \\
\text { lack of blinding }\end{array}$ \\
\hline
\end{tabular}

Collaborative care for depression and anxiety problems (Review)

Copyright @ 2012 The Cochrane Collaboration. Published by John Wiley \& Sons, Ltd. 
Unutzer 2002 (Continued)

Blinding of outcome assessment (detection Low risk

Assessor was not aware of treatment allocabias)

tion

All outcomes

Vera 2010

Methods

Study design: Randomised controlled trial

Participants

Setting: Primary care

Diagnosis: PHQ-9 and the depression scale of the Hopkins Symptom Checklist (HSCL) used. Meeting DSM-IV criteria for major depression based on their PHQ-9 score and a mean item score higher than 1.0 on the HSCL-20

Inclusion criteria: Aged 18 or older, willing to provide informed consent, and had any of the following health conditions diabetes, hypothyroidism, asthma, hypertension, chronic bronchitis, arthritis, heart disease, high cholesterol, or stroke. Spanish speaking and to have stated an intention to use the clinic as their main source of care in the next sixmonth period

Exclusion criteria: Serious suicidal risk or terminal illness, a history of bipolar or psychotic disorder or drug or alcohol abuse. Those receiving mental health treatment or applying for disability benefits

Age: Mean 55 years

Gender: $76 \%$ female

Ethnicity: Not stated

Country: Puerto Rico

Sample size (randomised): Total participants 179, intervention 89, control 90

Interventions

Intervention: Collaborative care

Contains the four elements of collaborative care:

1) a multi-professional approach to patient care: Primary care physician (PCP), counsellor/psychologist (CM), psychiatrist ( $\mathrm{MH}$ specialist)

2) a structured management plan: CMs provided patient education and offered ADs (Selective Serotonin Reuptake Inhibitor typically sertraline) or CBT (13 sessions with psychologist). CMs participated in the coordination of treatment initiation and monitored treatment adherence, side effects, and clinical response. CMs facilitated communication between the patient, PCP and MH specialist. In the medication treatment option, CMs provided follow-up based on depression severity. The $\mathrm{MH}$ specialist was responsible for prescribing and the CM forwarded recommendations to PCP. In CBT CMs provided mental health specialists with a progress report. Non-response resulted in a switch of modality or combined treatment

3) scheduled patient follow-ups: At least fortnightly initially and then monthly for up to six months (telephone or face-to-face). Additional contacts scheduled as needed 4) enhanced inter-professional communication: CMs facilitated communication between the patient, PCP and MH specialist. In the medication treatment, CMs had weekly case conference with $\mathrm{MH}$ specialist and forwarded treatment recommendations to the PCP

Control: Treatment as usual enhanced as patients were informed of their diagnosis and the mental health resources available. The CM encouraged patients to discuss depression treatment options with their PCP. A note was placed in the patient's medical record to

Collaborative care for depression and anxiety problems (Review) 
Vera 2010 (Continued)

notify PCPs

\begin{tabular}{ll}
\hline Outcomes & $\begin{array}{l}\text { Depression (HSCL): } 6 \text { months } \\
\text { Medication use: } 6 \text { months }\end{array}$ \\
\hline Notes & $\begin{array}{l}\text { CM: case manager; HSCL: Hopkins Symptom Checklist; MH: mental health; PCP: } \\
\text { primary care provider; PHQ-9: Patient Health Questionnaire-9 }\end{array}$ \\
\hline
\end{tabular}

Risk of bias

\begin{tabular}{|c|c|c|}
\hline Bias & Authors' judgement & Support for judgement \\
\hline $\begin{array}{l}\text { Random sequence generation (selection } \\
\text { bias) }\end{array}$ & Low risk & Computer generated in blocks of 20 \\
\hline Allocation concealment (selection bias) & Low risk & $\begin{array}{l}\text { Centrally prepared and opened numbered } \\
\text { opaque envelopes }\end{array}$ \\
\hline $\begin{array}{l}\text { Incomplete outcome data (attrition bias) } \\
\text { All outcomes }\end{array}$ & Unclear risk & $\begin{array}{l}\text { Short-term loss to follow-up based on pri- } \\
\text { mary depression outcome (HSCL) was: } \\
\text { overall } 12 / 179(7 \%), 6 / 89(7 \%) \text { interven- } \\
\text { tion and } 6 / 90(7 \%) \text { control. Reasons for } \\
\text { loss to follow-up not provided. Used inten- } \\
\text { tion-to-treat analysis }\end{array}$ \\
\hline Selective reporting (reporting bias) & Unclear risk & Insufficient information available to assess \\
\hline Other bias & Unclear risk & Insufficient information available to assess \\
\hline Implementation Integrity & Unclear risk & Insufficient information available to assess \\
\hline $\begin{array}{l}\text { Blinding of participants and personnel } \\
\text { (performance bias) } \\
\text { All outcomes }\end{array}$ & High risk & $\begin{array}{l}\text { Participants and personnel could not be } \\
\text { blinded, outcome likely to be influenced by } \\
\text { lack of blinding }\end{array}$ \\
\hline $\begin{array}{l}\text { Blinding of outcome assessment (detection } \\
\text { bias) } \\
\text { All outcomes }\end{array}$ & Low risk & $\begin{array}{l}\text { Assessor was not aware of treatment alloca- } \\
\text { tion }\end{array}$ \\
\hline
\end{tabular}

\section{Vlasveld 2011}

\begin{tabular}{l|l}
\hline Methods & Study design: Randomised controlled trial \\
\hline Participants & Setting: Community \\
& Diagnosis: Major depressive disorder assessed using the PHQ-9. Workers who reached \\
the cut-off score of 10 were contacted for the administration of a diagnostic interview. & Those who met the Diagnostic and Statistical Manual (DSM-IV) criteria for major \\
depressive disorder according to the mini - International Neuropsychiatric Interview
\end{tabular}


Vlasveld 2011 (Continued)

(MINI) were included

Inclusion criteria: Workers on the sick list for between 4 and 12 weeks who give informed consent

Exclusion criteria: Patients who are suicidal, psychotic or with a primary diagnosis of substance abuse or dependence, as assessed by the MINI interview, patients who do not have sufficient command of the Dutch language to fill in the questionnaires, patients who are pregnant, patients with a legal involvement against their employer, e.g. due to a conflict at work

Age: Not stated

Gender: $54 \%$ female

Ethnicity: Not stated

Country: The Netherlands

Sample size (randomised): Total participants 126, intervention 65, control 61

Interventions

Intervention: Collaborative care

Contains the four elements of collaborative care:

1) a multi-professional approach to patient care: Usual occupational physician (PCP), occupational physician (CM), psychiatrist (MH specialist)

2) a structured management plan: Contains the following elements: contracting (patient choice of treatment), adherence enhancing techniques (psychoeducation), manualguided self-help (focuses on behavioural activation, negative thoughts, return to work, and aspects of healthy lifestyle), problem solving therapy (PST), a workplace intervention (CM acts as mediator between patient and employer), active monitoring and, depending on patient preference, prescription of ADs according to a treatment algorithm. Patient starts with PST and the manual guided self-help, and some patients will also immediately start ADs. The workplace intervention will be fitted in during the first weeks of the intervention. Non-response will result in adding an extra 6 sessions of PST, or by adding $\mathrm{ADs}$ to the treatment plan or by increasing or changing the $\mathrm{AD}$. Continued non-response at 18 weeks will be referred to specialised mental health care and where medication is prescribed this will be handed over to GP

3) scheduled patient follow-ups: 9 contacts in 18 weeks (fortnightly). PST $=6$ sessions (plus extra 6 when required)

4) enhanced inter-professional communication: The PCP and CM communicated with each other with written informed consent of the patient. CM consulted $\mathrm{MH}$ specialist if needed and received regular group supervision with other CMs

Control: Treatment as usual in occupational health

Outcomes

Depression (PHQ-9): 3, 6, 9, 12 months

Medication use: 3, 6, 9, 12 months

Notes

AD: antidepressant; CM: case manager; GP: general practitioner; $\mathrm{MH}$ : mental health; PCP: primary care provider; PHQ-9: Patient Health Questionnaire; PST: problem solving therapy

Risk of bias

Bias

Authors' judgement

Support for judgement

Collaborative care for depression and anxiety problems (Review)

Copyright $\odot 2012$ The Cochrane Collaboration. Published by John Wiley \& Sons, Ltd. 
Vlasveld 2011 (Continued)

\begin{tabular}{|c|c|c|}
\hline $\begin{array}{l}\text { Random sequence generation (selection } \\
\text { bias) }\end{array}$ & Low risk & Computer generated \\
\hline Allocation concealment (selection bias) & Unclear risk & Insufficient information available to assess \\
\hline $\begin{array}{l}\text { Incomplete outcome data (attrition bias) } \\
\text { All outcomes }\end{array}$ & Unclear risk & $\begin{array}{l}\text { Short-term loss to follow-up based on } \\
\text { primary depression outcome (PHQ-9 re- } \\
\text { sponse } 50 \% \text { reduction) was: overall } 28 / \\
126(22 \%), 15 / 65(23 \%) \text { intervention and } \\
13 / 61(21 \%) \text { control. Reasons for loss } \\
\text { to follow-up not provided. Intention-to- } \\
\text { treat analysis reported, multiple imputa- } \\
\text { tion used to manage missing data }\end{array}$ \\
\hline Selective reporting (reporting bias) & Unclear risk & Insufficient information available to assess \\
\hline Other bias & Unclear risk & Insufficient information available to assess \\
\hline Implementation Integrity & Unclear risk & Insufficient information available to assess \\
\hline $\begin{array}{l}\text { Blinding of participants and personnel } \\
\text { (performance bias) } \\
\text { All outcomes }\end{array}$ & High risk & $\begin{array}{l}\text { Participants and personnel could not be } \\
\text { blinded, outcome likely to be influenced by } \\
\text { lack of blinding }\end{array}$ \\
\hline $\begin{array}{l}\text { Blinding of outcome assessment (detection } \\
\text { bias) } \\
\text { All outcomes }\end{array}$ & Low risk & $\begin{array}{l}\text { Assessor was not aware of treatment alloca- } \\
\text { tion }\end{array}$ \\
\hline
\end{tabular}

Waitzkin 2011

\begin{tabular}{ll}
\hline Methods & Study design: Cluster-randomised controlled trial \\
\hline Participants & $\begin{array}{l}\text { Setting: Primary care } \\
\text { Diagnosis: Diagnosis of depression on the Patient Health Questionnaire (PHQ) } \\
\text { Inclusion criteria: Not stated }\end{array}$ \\
Exclusion criteria: Suicidal or homicidal ideation (emergency care was provided to such \\
patients), acute bereavement, psychotic or bipolar depression, age under 18; and general \\
health status precluding the interview \\
Age: 18 years and over \\
Gender: $77 \%$ female \\
Ethnicity: Not stated, majority Hispanic \\
Country: United States \\
Sample size (randomised): Total participants 120, intervention unclear, control unclear \\
\hline Interventions & $\begin{array}{l}\text { Intervention: Enhanced care plus the promotoras contextual intervention } \\
\text { Contains the four elements of collaborative care: }\end{array}$ \\
1) a multi-professional approach to patient care: Primary care practitioners (PCP), pro- \\
motoras/lay health educators (CM)
\end{tabular}


Waitzkin 2011 (Continued)

2) a structured management plan: Same process as enhanced care initially as depression was assessed using measurement tool and findings provided to PCPs who then confirmed diagnosis and decided treatment plan (medication and/or counselling/therapy). The PCP and CM discuss plan, decide follow-up and CM leaves contact form in chart. In addition CM interviews patient on contextual sources of depression (unemployment or under-employment, housing, food, trauma) using a protocol and then assists the patient to deal with any issues

3) scheduled patient follow-ups: Every two months or dependent on need and decided between CM and PCP

4) enhanced inter-professional communication: CM provides PCP with depression score and depression guideline who reviews and determines treatment plan (medication and/or counselling/therapy). CM and PCP discuss plan and decide follow-up and communicate orally at least monthly. CMs complete a form at each contact. MH specialist provided consultation or saw patients where necessary

Control: Enhanced care as depression was assessed using measurement tool and findings provided to PCPs who then confirmed diagnosis and decided treatment plan (medication and/or counselling/therapy). The PCP and CM discuss plan, decide follow-up and CM leaves contact form in chart

\section{Risk of bias}

\section{Bias}

Random sequence generation (selection Low risk bias)

Allocation concealment (selection bias) Unclear risk

Incomplete outcome data (attrition bias) Unclear risk All outcomes

Selective reporting (reporting bias)

Other bias

High risk

Implementation Integrity
Unclear risk
Unclear risk

\section{Support for judgement}

Coin-toss (three out of five)

Insufficient information available to assess

Short-term loss to follow-up based on primary depression outcome (PHQ16 symptom count) was unclear Reasons for loss to follow-up not provided

Intention to treat not reported

Insufficient information available to assess

Case managers collaborated with PCPs to develop treatment plans for patients in the control group

Insufficient information available to assess 
Waitzkin 2011 (Continued)

Blinding of participants and personnel High risk (performance bias)

All outcomes
Participants and personnel could not be blinded, outcome likely to be influenced by lack of blinding

Insufficient information available to assess

Blinding of outcome assessment (detection Unclear risk

bias)

All outcomes

Wells 2000a

\begin{tabular}{ll}
\hline Methods & Study design: Cluster-randomised controlled trial \\
\hline Participants & Setting: Primary care \\
Diagnosis: Depression measured by the Composite International Diagnostic Interview \\
(CIDI) and items assessing depressed symptoms in the past month. Defined patients \\
as having probable disorder if they had 2 weeks or more of depressed mood or loss of \\
interest in pleasurable activities during the last year or persistent depression over the year, \\
plus having at least 1 week of depression in the last 30 days \\
Inclusion criteria: Patients who intended to use the clinic as a source of care for the next \\
12 months \\
Exclusion criteria: Younger than 18 years, had an acute medical emergency, did not speak \\
English or Spanish, or did not have either insurance or a public-pay arrangement that \\
covered care delivered by the mental health specialists in the interventions. In the pilot \\
month for the first site, patients screening positive for bipolar disorder or alcoholism \\
were excluded, but not for the main study \\
Age: Mean 43.7 (SD 15) years \\
Gender: $71 \%$ female \\
Ethnicity: $57 \%$ White \\
Country: United States \\
Sample size (randomised): 31 primary care clinics, intervention 15, control 16; Total \\
participants 867 , intervention 424 , control 443 \\
\hline
\end{tabular}

Interventions

Intervention: Quality Improvement medication (QI-meds)

Contains the four elements of collaborative care:

1) a multi-professional approach to patient care: Primary care clinicians (PCP), nurse (CM), psychiatrist ( $\mathrm{MH}$ specialist)

2) a structured management plan: The programme included 1) institutional commitment, 2) training local leaders to implement interventions 3) training of CMs (patient education and activation based on a written manual and videotape) 4) patient identification. CMs assessed, educated, and activated) patients, sharing the information with PCPs who formulated a treatment plan with the patient. QI-meds involved CMs providing follow-up assessments and supporting adherence and facilitating referral for local psychotherapy where necessary

3) scheduled patient follow-ups: 8 sessions ( 2 and 4 weeks then monthly for 6 months) half were also randomised to receive 3 further sessions in preceding 6 months

4) enhanced inter-professional communication: CMs provided assessment information to PCP who formulated a treatment plan with the patient. PCPs reviewed CM written reports and met with patients when necessary. $\mathrm{MH}$ specialists reviewed $\mathrm{CM}$ reports and 
Wells 2000a

met with patients with poor treatment response at 6-8 weeks

Control: Treatment as usual enhanced as treatment guidelines, with quick reference guides for clinicians, were sent to medical directors. Patients were told they could inform the PCP of allocation/diagnosis

\begin{tabular}{ll} 
Outcomes & $\begin{array}{l}\text { Depression (Probable depression): 6, 12, 24, } 57 \text { months } \\
\text { Medication use: 6, 12, 18, 24 months } \\
\text { Quality of Life (mental and physical health): 6, 12, 18, 24, } 57 \text { months, } 9 \text { years }\end{array}$ \\
\hline Notes & $\begin{array}{l}\text { CIDI: Composite International Diagnostic Interview; CM: case manager; MH: mental } \\
\text { health; PCP: primary care provider }\end{array}$
\end{tabular}

Risk of bias

\begin{tabular}{|c|c|c|}
\hline Bias & Authors' judgement & Support for judgement \\
\hline $\begin{array}{l}\text { Random sequence generation (selection } \\
\text { bias) }\end{array}$ & Low risk & $\begin{array}{l}\text { Clusters were organised into blocks of } \\
3 \text {, blocks were randomised using random } \\
\text { numbers table }\end{array}$ \\
\hline Allocation concealment (selection bias) & Unclear risk & Insufficient information available to assess \\
\hline $\begin{array}{l}\text { Incomplete outcome data (attrition bias) } \\
\text { All outcomes }\end{array}$ & Unclear risk & $\begin{array}{l}\text { Short-term loss to follow-up based on pri- } \\
\text { mary depression outcome (Probable de- } \\
\text { pression) was: overall } 113 / 867(13 \%), 56 / \\
424(13 \%) \text { intervention and } 57 / 443(13 \%) \\
\text { control. Reasons for loss to follow-up not } \\
\text { provided by group. Used intention-to-treat } \\
\text { analysis }\end{array}$ \\
\hline Selective reporting (reporting bias) & Low risk & $\begin{array}{l}\text { Protocol available and all prespecified out- } \\
\text { comes reported }\end{array}$ \\
\hline Other bias & Unclear risk & Insufficient information available to assess \\
\hline Implementation Integrity & Unclear risk & Insufficient information available to assess \\
\hline $\begin{array}{l}\text { Blinding of participants and personnel } \\
\text { (performance bias) } \\
\text { All outcomes }\end{array}$ & High risk & $\begin{array}{l}\text { Participants and personnel could not be } \\
\text { blinded, outcome likely to be influenced by } \\
\text { lack of blinding }\end{array}$ \\
\hline $\begin{array}{l}\text { Blinding of outcome assessment (detection } \\
\text { bias) } \\
\text { All outcomes }\end{array}$ & Unclear risk & Insufficient information available to assess \\
\hline
\end{tabular}


Methods
Participants

Study design: Cluster-randomised controlled trial

Setting: Primary care

Diagnosis: Depression measured by the Composite International Diagnostic Interview (CIDI) and items assessing depressed symptoms in the past month. Defined patients as having probable disorder if they had 2 weeks or more of depressed mood or loss of interest in pleasurable activities during the last year or persistent depression over the year, plus having at least 1 week of depression in the last 30 days

Inclusion criteria: Patients who intended to use the clinic as a source of care for the next 12 months

Exclusion criteria: Younger than 18 years, had an acute medical emergency, did not speak English or Spanish, or did not have either insurance or a public-pay arrangement that covered care delivered by the mental health specialists in the interventions. In the pilot month for the first site, patients screening positive for bipolar disorder or alcoholism were excluded, but not for the main study

Age: Mean 43.7 (SD 15) years

Gender: $71 \%$ female

Ethnicity: $57 \%$ white

Country: United States

Sample size (randomised): 31 primary care clinics, intervention 15, control 16; Total participants 932, intervention 489, control 443

Interventions

Intervention: Quality improvement therapy (QI-therapy)

Contains the four elements of collaborative care:

1) a multi-professional approach to patient care: Primary care clinicians (PCP), psychotherapist (CM), psychologist (MH specialist)

2) a structured management plan: The programme included 1) institutional commitment, 2) training local leaders to implement interventions 3) training of CMs (patient education and activation based on a written manual and videotape) 4) patient identification. CMs assessed, educated, and activated) patients, sharing the information with PCPs who formulated a treatment plan with the patient. QI-therapy involved psychotherapists providing manualised individual or group CBT which consisted of 3 modules of 4 sessions with a recommendation for repeating the first one. Patients were provided with a manual for recording clinical progress and homework assignments

3) scheduled patient follow-ups: 12-16 individual or group sessions (4 sessions for those with minor depression)

4) enhanced inter-professional communication: CM used feedback forms to communicate with PCPs at beginning, at termination and at module breaks

Control: Treatment as usual enhanced as treatment guidelines, with quick reference guides for clinicians, were sent to medical directors. Patients were told they could inform the PCP of allocation/diagnosis

Outcomes

Depression (Probable depression): 6, 12, 24, 57 months

Medication use: 6, 12, 18, 24 months

Quality of Life (mental and physical health): 6, 12, 18, 24, 57 months, 9 years

Notes

CBT: cognitive behaviour therapy; CIDI: Composite International Diagnostic Interview; CM: case manager; $\mathrm{MH}$ : mental health; PCP: primary care provider 
Wells 2000b (Continued)

\section{Risk of bias}

\begin{tabular}{|c|c|c|}
\hline Bias & Authors' judgement & Support for judgement \\
\hline $\begin{array}{l}\text { Random sequence generation (selection } \\
\text { bias) }\end{array}$ & Low risk & $\begin{array}{l}\text { Clusters were organised into blocks of } \\
3 \text {, blocks were randomised using random } \\
\text { numbers table }\end{array}$ \\
\hline Allocation concealment (selection bias) & Unclear risk & Insufficient information available to assess \\
\hline $\begin{array}{l}\text { Incomplete outcome data (attrition bias) } \\
\text { All outcomes }\end{array}$ & Unclear risk & $\begin{array}{l}\text { Short-term loss to follow-up based on pri- } \\
\text { mary depression outcome (Probable de- } \\
\text { pression) was: overall 144/932 (15\%), } 87 / \\
489(18 \%) \text { intervention and } 57 / 443(13 \%) \\
\text { control. Reasons for loss to follow-up not } \\
\text { provided by group. Used intention-to-treat } \\
\text { analysis }\end{array}$ \\
\hline Selective reporting (reporting bias) & Low risk & $\begin{array}{l}\text { Protocol available and all prespecified out- } \\
\text { comes reported }\end{array}$ \\
\hline Other bias & Unclear risk & Insufficient information available to assess \\
\hline Implementation Integrity & Unclear risk & Insufficient information available to assess \\
\hline $\begin{array}{l}\text { Blinding of participants and personnel } \\
\text { (performance bias) } \\
\text { All outcomes }\end{array}$ & High risk & $\begin{array}{l}\text { Participants and personnel could not be } \\
\text { blinded, outcome likely to be influenced by } \\
\text { lack of blinding }\end{array}$ \\
\hline $\begin{array}{l}\text { Blinding of outcome assessment (detection } \\
\text { bias) } \\
\text { All outcomes }\end{array}$ & Unclear risk & Insufficient information available to assess \\
\hline
\end{tabular}

Wilkinson 1993

Methods

Participants
Study design: Pilot randomised controlled trial

Setting: Primary care

Diagnosis: Depressive disorder judged by the General Practitioner to require treatment with antidepressant medication

Inclusion criteria: Males and females above the age of consent

Exclusion criteria: Use of tricyclic antidepressants was not permitted for the 28 days preceding entry to the study

Age: Mean 46 years

Gender: $74 \%$ female

Ethnicity: Not stated

Country: United Kingdom

Sample size (randomised): Total participants 61, intervention 30, control 31 
Wilkinson 1993 (Continued)

\begin{tabular}{|c|c|c|}
\hline Interventions & \multicolumn{2}{|c|}{$\begin{array}{l}\text { Intervention: Dothiepin plus Practice Nurse supplement } \\
\text { Contains the four elements of collaborative care: } \\
\text { 1) a multi-professional approach to patient care: General practitioner (PCP), practice } \\
\text { nurse (CM), } \\
\text { 2) a structured management plan: CM aimed to enhance treatment adherence to med- } \\
\text { ication by discussion and encouragement particularly by providing explanation and re- } \\
\text { assurance about pharmacological adverse events of medication } \\
\text { 3) scheduled patient follow-ups: } 5 \text { face-to-face sessions (days } 0,7,14,28,56) \\
\text { 4) enhanced inter-professional communication: CM care under the supervision of the } \\
\text { PCP } \\
\text { Control: Treatment as usual plus dothiepin }\end{array}$} \\
\hline Outcomes & \multicolumn{2}{|c|}{$\begin{array}{l}\text { Depression (Global clinical effectiveness): } 2 \text { months } \\
\text { Medication use: } 2 \text { months }\end{array}$} \\
\hline Notes & \multicolumn{2}{|c|}{ CM: case manager; $\mathrm{MH}$ : mental health; PCP: primary care provider } \\
\hline \multicolumn{3}{|l|}{ Risk of bias } \\
\hline Bias & Authors' judgement & Support for judgement \\
\hline $\begin{array}{l}\text { Random sequence generation (selection } \\
\text { bias) }\end{array}$ & Unclear risk & Random allocation in blocks of 4 \\
\hline Allocation concealment (selection bias) & Unclear risk & $\begin{array}{l}\text { Sealed pre-packed study protocols were se- } \\
\text { lected in turn }\end{array}$ \\
\hline $\begin{array}{l}\text { Incomplete outcome data (attrition bias) } \\
\text { All outcomes }\end{array}$ & Unclear risk & $\begin{array}{l}\text { Short-term loss to follow-up based on pri- } \\
\text { mary depression outcome (Global clinical } \\
\text { effectiveness) was: overall } 9 / 61(15 \%), 5 / 30 \\
(17 \%) \text { intervention and } 4 / 31(13 \%) \text { con- } \\
\text { trol. Reasons for loss to follow-up provided, } \\
\text { with similar reasons across groups. Inten- } \\
\text { tion to treat not reported }\end{array}$ \\
\hline Selective reporting (reporting bias) & Unclear risk & Insufficient information available to assess \\
\hline Other bias & Unclear risk & Insufficient information available to assess \\
\hline Implementation Integrity & Unclear risk & Insufficient information available to assess \\
\hline $\begin{array}{l}\text { Blinding of participants and personnel } \\
\text { (performance bias) } \\
\text { All outcomes }\end{array}$ & High risk & $\begin{array}{l}\text { Participants and personnel could not be } \\
\text { blinded, outcome likely to be influenced by } \\
\text { lack of blinding }\end{array}$ \\
\hline $\begin{array}{l}\text { Blinding of outcome assessment (detection } \\
\text { bias) } \\
\text { All outcomes }\end{array}$ & High risk & $\begin{array}{l}\text { Assessor was potentially aware of treatment } \\
\text { allocation }\end{array}$ \\
\hline
\end{tabular}

Collaborative care for depression and anxiety problems (Review)

Copyright @ 2012 The Cochrane Collaboration. Published by John Wiley \& Sons, Ltd. 
Methods

Participants

Interventions
Study design: Randomised controlled trial

Setting: Specialist, primary care

Diagnosis: Diagnosis of major or minor depression. Those endorsing either the depressed mood or the anhedonia item or those with scores $\geq 5$ on the PHQ-9 regardless of items endorsed were administered the structured clinical interview for Diagnostic and Statistical Manual of Mental Disorders (DSM-IV) to confirm diagnosis

Inclusion criteria: Adults 18 years and older with ischaemic stroke, no severe language impairment, no severe cognitive impairment, able to speak and understand English, had a telephone, and who had a life expectancy of at least 6 months

Exclusion criteria: Hemorrhagic stroke, active psychosis, suicidality, or substance abuse; those currently taking a monoamine oxidase inhibitor; and women pregnant at the time of stroke. Severe aphasia, pre-existing dementia or failed cognitive screening

Age: Mean 60 years

Gender: $55 \%$ female

Ethnicity: 61\% white

Country: United States

Sample size (randomised): Total participants 188, intervention 94, control 94

Intervention: Care management (Activate-Initiate-Monitor)

Contains the four elements of collaborative care:

1) a multi-professional approach to patient care: Primary care provider/neurologist (PCP) , nurse (CM), study physician (Specialist)

2) a structured management plan: CM intervention consisted of 3 steps: (1) Activating stroke survivors and their families to understand and accept depression diagnosis and treatment (a structured psychoeducation session), (2) Initiating ADs (CM recommends PCP prescribe AD using algorithm, typically a Selective Serotonin Reuptake Inhibitor); and (3) Monitoring treatment effectiveness (scripted bimonthly telephone calls to assess depression symptoms, medication side effects, and adherence). Non-response after 4 weeks led to increase in $\mathrm{AD}$ dose. CMs had weekly meetings with Specialist and treatment recommendations were fed back to PCP by CM

3) scheduled patient follow-ups: 7 sessions in 12 weeks (One face-to-face and bimonthly telephone calls)

4) enhanced inter-professional communication: $\mathrm{CMs}$ recommended $\mathrm{AD}$ to $\mathrm{PCP}$ and met with Specialist weekly and fed back treatment recommendations to PCP

Control: Patients received an identical number of baseline and telephone sessions to serve as a control for an attention effect. Instead of depression, these sessions focused on recognition and monitoring of stroke symptoms and risks

Depression (HAMD): 6, 12 months

Notes

AD: antidepressant; $\mathrm{CM}$ : case manager; $\mathrm{MH}$ : mental health; $\mathrm{PCP}$ : primary care provider; PHQ-9: Patient Health Questionnaire

Risk of bias

Bias

Authors' judgement 


\begin{tabular}{|c|c|c|}
\hline $\begin{array}{l}\text { Random sequence generation (selection } \\
\text { bias) }\end{array}$ & Low risk & $\begin{array}{l}\text { Computer generated in blocks of } 2 \text { and } 4 \\
\text { (randomly selected) }\end{array}$ \\
\hline Allocation concealment (selection bias) & Low risk & $\begin{array}{l}\text { Sealed opaque envelopes prepared by an } \\
\text { individual not involved in patient recruit- } \\
\text { ment and opened sequentially }\end{array}$ \\
\hline $\begin{array}{l}\text { Incomplete outcome data (attrition bias) } \\
\text { All outcomes }\end{array}$ & Low risk & $\begin{array}{l}\text { Short-term loss to follow-up based on pri- } \\
\text { mary depression outcome (HAMD) was: } \\
\text { overall } 6 / 188(3 \%), 5 / 94(5 \%) \text { interven- } \\
\text { tion and } 1 / 94(1 \%) \text { control. Reasons for } \\
\text { loss to follow-up provided, with similar rea- } \\
\text { sons across groups. Used intention-to-treat } \\
\text { analysis }\end{array}$ \\
\hline Selective reporting (reporting bias) & Unclear risk & Insufficient information available to assess \\
\hline Other bias & Unclear risk & Insufficient information available to assess \\
\hline Implementation Integrity & Unclear risk & Insufficient information available to assess \\
\hline $\begin{array}{l}\text { Blinding of participants and personnel } \\
\text { (performance bias) } \\
\text { All outcomes }\end{array}$ & High risk & $\begin{array}{l}\text { Participants and personnel could not be } \\
\text { blinded, outcome likely to be influenced by } \\
\text { lack of blinding }\end{array}$ \\
\hline $\begin{array}{l}\text { Blinding of outcome assessment (detection } \\
\text { bias) } \\
\text { All outcomes }\end{array}$ & Low risk & $\begin{array}{l}\text { Assessor was not aware of treatment alloca- } \\
\text { tion }\end{array}$ \\
\hline
\end{tabular}

\section{Yeung 2010}

\begin{tabular}{ll} 
Methods & Study design: Randomised controlled trial \\
\hline Participants & Setting: Primary care \\
& Diagnosis: Depression measured by a score of 10 or above on the Chinese Bilingual \\
& Patient Health Questionnaire-9 (CB-PHQ-9) \\
& Inclusion criteria: Chinese American adults (18 years or older) who attended the primary \\
& care clinic \\
& Exclusion criteria: Patients with unstable medical conditions, a high risk of suicide, \\
& psychotic disorders, dysthymic disorder, bipolar disorder, and substance use disorders \\
& Age: Mean 49.5 years \\
& Gender: $68 \%$ female \\
Ethnicity: $100 \%$ Chinese American & Country: United States \\
& Sample size (randomised): Total participants 100, intervention 55 , control 45
\end{tabular}




\begin{tabular}{|c|c|c|}
\hline Interventions & \multicolumn{2}{|c|}{$\begin{array}{l}\text { Intervention: Care management } \\
\text { Contains the four elements of collaborative care: } \\
\text { 1) a multi-professional approach to patient care: Primary care provider (PCP), bilingual } \\
\text { care manager (CM), psychiatrist (MH specialist) } \\
\text { 2) a structured management plan: CMs established rapport, explained the roles of the } \\
\mathrm{CM} \text {, and provided culturally sensitive education on major depression. Follow-ups fo- } \\
\text { cused on monitoring of depressive symptoms, adherence to medication treatment, man- } \\
\text { agement of adverse events, and knowledge of self-management strategies } \\
\text { 3) scheduled patient follow-ups: } 8 \text { sessions (one face-to-face and } 7 \text { telephone at weeks 2, } \\
\text { 4, 8, 12, 16, 20, 24) } \\
\text { 4) enhanced inter-professional communication: CMs served as a link between patients, } \\
\text { PCPs, and consulting MH specialist. CMs received weekly supervision from MH spe- } \\
\text { cialist who also had consultations with patients and their PCPs when required } \\
\text { Control: Treatment as usual enhanced as patients with major depressive disorder were } \\
\text { encouraged to seek treatment from their PCP, who would receive a letter about the } \\
\text { patient's diagnosis and a recommended treatment plan }\end{array}$} \\
\hline Outcomes & \multicolumn{2}{|c|}{ Depression (HAMD17): Most recent } \\
\hline Notes & \multicolumn{2}{|c|}{ CM: case manager; $\mathrm{MH}$ : mental health; PCP: primary care provider } \\
\hline \multicolumn{3}{|l|}{ Risk of bias } \\
\hline Bias & Authors' judgement & Support for judgement \\
\hline $\begin{array}{l}\text { Random sequence generation (selection } \\
\text { bias) }\end{array}$ & Low risk & Computer generated \\
\hline Allocation concealment (selection bias) & Unclear risk & Insufficient information available to assess \\
\hline $\begin{array}{l}\text { Incomplete outcome data (attrition bias) } \\
\text { All outcomes }\end{array}$ & Unclear risk & $\begin{array}{l}\text { Short-term loss to follow-up based on pri- } \\
\text { mary depression outcome (Response } 50 \% \\
\text { reduction HAMD 17) was: overall } 25 / \\
100(25 \%), 14 / 55(25 \%) \text { intervention and } \\
11 / 45(24 \%) \text { control. Reasons for loss to } \\
\text { follow-up not provided. Intention-to-treat } \\
\text { analysis not reported, no description of } \\
\text { methods to manage missing data }\end{array}$ \\
\hline Selective reporting (reporting bias) & Unclear risk & Insufficient information available to assess \\
\hline Other bias & Unclear risk & Insufficient information available to assess \\
\hline Implementation Integrity & Unclear risk & Insufficient information available to assess \\
\hline $\begin{array}{l}\text { Blinding of participants and personnel } \\
\text { (performance bias) } \\
\text { All outcomes }\end{array}$ & High risk & $\begin{array}{l}\text { Participants and personnel could not be } \\
\text { blinded, outcome likely to be influenced by } \\
\text { lack of blinding }\end{array}$ \\
\hline
\end{tabular}


Methods

Participants
Study design: Pilot randomised controlled trial

Setting: Specialist, primary care

Diagnosis: The Centre for Epidemiological Studies Depression Scale (CES-D), a 20item self-report instrument to measure levels of depressive symptoms was used. Levels of PTSD symptoms were assessed using the civilian version of the Post-Traumatic Stress Disorder Checklist (PCL-C)

Inclusion criteria: Hospitalised motor vehicle crash or assault survivors between the ages of 14-65, who were English speaking

Exclusion criteria: Patients who sustained severe injuries (i.e. Maximum Abbreviated Injury Scale (AIS) score $\geq 5$ ), were excluded

Age: Mean 33.9 years

Gender: $42 \%$ female

Ethnicity: 62\% white

Country: United States

Sample size (randomised): Total participants 34, intervention 16, control 18

Interventions

Intervention: Collaborative care

Contains the four elements of collaborative care:

1) a multi-professional approach to patient care: Surgical/primary care provider (PCP), nurse specialist/psychiatrist (CM), multidisciplinary team (MH specialist)

2) a structured management plan: CMs were instructed to develop a therapeutic relationship and follow patients for 4 months through primary care outpatient appointments and community rehabilitation. CM involved collaborative problem definition and shared patient-provider treatment planning (based on post-traumatic concerns). CMs intervened in resolution of these concerns and also provided a psychotherapy module specifically targeting post-traumatic distress and substance use. The psychoeducational component began with a review of the traumatic event, followed by a discussion of related emotions, cognitions, and possible future post-traumatic symptoms, and closed with suggested coping strategies including algorithms for contacting the CM. The motivational enhancement techniques focused on the evaluation of readiness to change and implementation of a motivational interview targeting post-traumatic alcohol and drug use

3) scheduled patient follow-ups: Face-to-face whilst inpatient and then follow for 4 months (typically telephone)

4) enhanced inter-professional communication: After discharge CMs had regular contact with PCPs via telephone especially in first month. CMs met with $\mathrm{MH}$ specialists weekly to review CM written records

Control: Treatment as usual for post-traumatic care

Outcomes

Anxiety (PTSD): 1, 4 months

Quality of Life (mental and physical health): 1, 4 months 
Zatzick 2001 (Continued)

\begin{tabular}{ll} 
Notes & CM: case manager; $\mathrm{MH}$ : mental health; PCP: primary care provider \\
\hline
\end{tabular}

\section{Risk of bias}

\begin{tabular}{|c|c|c|}
\hline Bias & Authors' judgement & Support for judgement \\
\hline $\begin{array}{l}\text { Random sequence generation (selection } \\
\text { bias) }\end{array}$ & Low risk & $\begin{array}{l}\text { Random numbers table used to determine } \\
\text { which patients to approach. Once recruited } \\
\text { an individual not involved in patient re- } \\
\text { cruitment randomised patients in blocks of } \\
6\end{array}$ \\
\hline
\end{tabular}

Allocation concealment (selection bias) Low risk

Incomplete outcome data (attrition bias) Unclear risk All outcomes
Allocation conducted by an individual not involved in patient recruitment

Short-term loss to follow-up based on primary anxiety outcome (PTSD) was: overall 8/34 (24\%), 4/16 (25\%) intervention and $4 / 18(22 \%)$ control. Reasons for loss to follow-up not provided. Intention-to-treat analysis reported, no description of methods to manage missing data

\begin{tabular}{l|ll}
\hline Selective reporting (reporting bias) & Unclear risk & Insufficient information available to assess \\
\hline Other bias & Unclear risk & Insufficient information available to assess \\
\hline
\end{tabular}

Implementation Integrity Low risk

Attempts were made to assess implementation integrity (e.g. direct observation or rating of tapes)

Blinding of participants and personnel High risk (performance bias)

All outcomes

Participants and personnel could not be blinded, outcome likely to be influenced by lack of blinding

Assessor was not aware of treatment allocation

Blinding of outcome assessment (detection Low risk bias)

All outcomes

\section{Zatzick 2004}

Methods

Participants
Study design: Randomised controlled trial

Setting: Specialist, primary care

Diagnosis: PTSD Checklist Civilian Version (PCL) and the Centre for Epidemiological Studies Depression Scale (CES-D) were used. Patients were included if they had a PCL score, $\geq 45$ and/or a CESD-D score, $\geq 16$

Inclusion criteria: English-speaking survivors of intentional and unintentional injuries, 
18 years and older, who lived within 50 miles of the trauma centre

Exclusion criteria: Participants were required to have a Glasgow Coma Scale score of 15 and a score of at least 7 on the 2 Mini- Mental State Examination items that assess orientation to location and date. Patients with severe injuries that prevented participation were excluded from the study. Patients who had self-inflicted injuries or active psychosis, who were currently incarcerated, or who had recent histories of violence were also excluded

Age: Mean 40.8 years

Gender: $33 \%$ female

Ethnicity: 66\% White

Country: United States

Sample size (randomised): Total participants 121, intervention 60, control 61

Interventions
Intervention: Collaborative care

Contains the four elements of collaborative care:

1) a multi-professional approach to patient care: Primary care provider (PCP), masters level case manager (CM), psychologist/psychiatrist (MH specialist)

2) a structured management plan: The intervention combined case management and psychopharmacological and psychotherapeutic treatments as a stepped-care procedure. For the first 6 months after injury, all patients received continuous case management delivered by CM. CMs and other intervention team members developed a comprehensive care plan that addressed medical and psychosocial complications and coordinated care across surgical inpatient, primary care outpatient, specialty mental health, and community service settings. Patients had 24 hour 7 day a week access to CM pager and CMs developed a therapeutic alliance that facilitated the delivery of evidence-based interventions for alcohol abuse and PTSD. The CM motivational intervention consisted of an inpatient session followed by as-needed booster sessions (test results, pros and cons of alcohol, importance of change, specific goals for alcohol, and action plans). Three months after the injury patients with PTSD were given preference of CBT, pharmacotherapy, or combined treatment (delivered by expert psychotherapy and pharmacotherapy consultants). The CBT intervention included psychoeducation, muscle relaxation, cognitive restructuring, and graded exposure. The psychopharmacological intervention consisted of an initial psychiatric evaluation and medication targeting PTSD. CMs provided education about the diagnosis and facilitated the entry of patients into evidence-based treatments. During the evidence-based PTSD intervention, CMs performed brief assessments of adherence to medication therapy and symptom relapse. The stepped-care procedure included relapse prevention and community integration components. From 6 to 12 months after the injury, non-responsive patients received combination treatments and CMs periodically reassessed symptoms, function, and rehabilitation

3) scheduled patient follow-ups: Continuous for 6 months and then periodic 6-12 months for those that remained symptomatic

4) enhanced inter-professional communication: CMs coordinated linkages and interfaced with PCPs and met with MH specialist weekly to review cases and protocol procedures

Control: Treatment as usual for post-traumatic care 


\section{Risk of bias}

\begin{tabular}{|c|c|c|}
\hline Bias & Authors' judgement & Support for judgement \\
\hline $\begin{array}{l}\text { Random sequence generation (selection } \\
\text { bias) }\end{array}$ & Low risk & Computer generated \\
\hline Allocation concealment (selection bias) & Unclear risk & Insufficient information available to assess \\
\hline $\begin{array}{l}\text { Incomplete outcome data (attrition bias) } \\
\text { All outcomes }\end{array}$ & Unclear risk & $\begin{array}{l}\text { Short-term loss to follow-up based on pri- } \\
\text { mary anxiety outcome (DSM-IV PTSD } \\
\text { from PTSD checklist) was: overall } 17 / 121 \\
(14 \%), 8 / 60(13 \%) \text { intervention and } 9 / 61 \\
(15 \%) \text { control. Reasons for loss to follow- } \\
\text { up not provided. Used intention-to-treat } \\
\text { analysis }\end{array}$ \\
\hline Selective reporting (reporting bias) & Unclear risk & Insufficient information available to assess \\
\hline Other bias & Unclear risk & Insufficient information available to assess \\
\hline Implementation Integrity & Unclear risk & Insufficient information available to assess \\
\hline $\begin{array}{l}\text { Blinding of participants and personnel } \\
\text { (performance bias) } \\
\text { All outcomes }\end{array}$ & High risk & $\begin{array}{l}\text { Participants and personnel could not be } \\
\text { blinded, outcome likely to be influenced by } \\
\text { lack of blinding }\end{array}$ \\
\hline $\begin{array}{l}\text { Blinding of outcome assessment (detection } \\
\text { bias) } \\
\text { All outcomes }\end{array}$ & Unclear risk & Insufficient information available to assess \\
\hline
\end{tabular}

\section{Characteristics of excluded studies [ordered by study ID]}

\begin{tabular}{ll}
\hline Study & Reason for exclusion \\
\hline Akerblad 2003 & $\begin{array}{l}\text { Types of intervention: Does not meet criteria for a multi-professional approach to patient care (only } \\
\text { one health professional involved) }\end{array}$ \\
\hline Al-Saffar 2005 & $\begin{array}{l}\text { Types of intervention: Does not meet criteria for a multi-professional approach to patient care (no } \\
\text { primary care provider) }\end{array}$ \\
\hline Bolton 2001 & Types of intervention: Does not meet criteria for enhanced inter-professional communication
\end{tabular}


(Continued)

\begin{tabular}{|c|c|}
\hline Braamse 2010 & $\begin{array}{l}\text { Types of intervention: Does not meet criteria for a multi-professional approach to care (no primary } \\
\text { care provider) }\end{array}$ \\
\hline Britian 1999 & Types of participants: Less than $50 \%$ of participants had depression and/or anxiety at baseline \\
\hline Brook 2003 & $\begin{array}{l}\text { Types of intervention: Does not meet criteria for a multi-professional approach to patient care (no } \\
\text { primary care provider) }\end{array}$ \\
\hline Callahan 1994 & $\begin{array}{l}\text { Types of intervention: Does not meet criteria for a multi-professional approach to patient care (only } \\
\text { one health professional involved) }\end{array}$ \\
\hline Callahan 2006 & Types of participants: Less than $50 \%$ of participants had depression and/or anxiety at baseline \\
\hline Cheok 2003 & Types of intervention: Does not meet criteria for scheduled patient follow-ups \\
\hline Coleman 1999 & Types of participants: Less than $50 \%$ of participants had depression and/or anxiety at baseline \\
\hline Dobscha 2006 & Types of intervention: Does not meet criteria for scheduled patient follow-ups \\
\hline Dobscha 2008 & Types of participants: Less than $50 \%$ of participants had depression and/or anxiety at baseline \\
\hline Dobscha 2009 & Types of participants: Less than $50 \%$ of participants had depression and/or anxiety at baseline \\
\hline Dozeman 2007 & Types of participants: Participants were recruited and/or treated in specialist mental health setting \\
\hline Gellis 2010 & Types of intervention: Does not meet criteria for enhanced inter-professional communication \\
\hline Geron 2006 & Types of participants: Less than $50 \%$ of participants had depression and/or anxiety at baseline \\
\hline Hees 2010 & Types of participants: Participants were recruited and/or treated in specialist mental health setting \\
\hline Katon 1992 & Types of intervention: Does not meet criteria for scheduled patient follow-ups \\
\hline Kroenke 2009 & $\begin{array}{l}\text { Types of intervention: Does not meet criteria for a multi-professional approach to patient care (no } \\
\text { primary care provider) }\end{array}$ \\
\hline Lyles 2003 & Types of participants: Less than $50 \%$ of participants had depression and/or anxiety at baseline \\
\hline Meglic 2010 & Types of studies: Not a randomised controlled trial (systematic alternating order without blinding) \\
\hline Mudge 2011 & Types of intervention: Intervention not focused on depression or anxiety \\
\hline Oslin 2004 & $\begin{array}{l}\text { Types of intervention: Does not meet criteria for a multi-professional approach to care (no primary } \\
\text { care provider) }\end{array}$ \\
\hline Peveler 1999 & $\begin{array}{l}\text { Types of intervention: Does not meet criteria for a multi-professional approach to patient care (no } \\
\text { primary care provider) }\end{array}$ \\
\hline
\end{tabular}

Collaborative care for depression and anxiety problems (Review)

Copyright $\odot 2012$ The Cochrane Collaboration. Published by John Wiley \& Sons, Ltd. 
(Continued)

\begin{tabular}{|c|c|}
\hline Pols 2008 & Types of intervention: Primary care provider delivered intervention not case manager \\
\hline Raue 2009 & Types of studies: Not a randomised controlled trial \\
\hline Rickles 2005 & $\begin{array}{l}\text { Types of intervention: Does not meet criteria for a multi-professional approach to patient care (no } \\
\text { primary care provider) }\end{array}$ \\
\hline Riegel 2006 & Types of intervention: Intervention not focused on depression \\
\hline Seekles 2009 & $\begin{array}{l}\text { Types of intervention: Stepped care - collaborative care intervention not available for all patients in } \\
\text { treatment arm }\end{array}$ \\
\hline Simon 2006 & $\begin{array}{l}\text { Types of intervention: Does not meet criteria for a multi-professional approach to care (no primary } \\
\text { care provider) }\end{array}$ \\
\hline Sirey 2010 & Types of intervention: Does not meet criteria for enhanced inter-professional communication \\
\hline Stevens 2009 & Types of participants: Participants were recruited and/or treated in specialist mental health setting \\
\hline van der Feltz-Cornelis 2006 & Types of intervention: Does not meet criteria for scheduled patient follow-ups \\
\hline van der Feltz-Cornelis 2010 & Types of intervention: Does not meet criteria for scheduled patient follow-ups \\
\hline Van't Veer-Tazelaar 2009 & $\begin{array}{l}\text { Types of intervention: Stepped care - collaborative care intervention not available for all patients in } \\
\text { treatment arm }\end{array}$ \\
\hline Wang 2007 & Types of participants: Participants were recruited and/or treated in specialist mental health setting \\
\hline Whooley 2000 & Types of intervention: Does not meet criteria for enhanced inter-professional communication \\
\hline
\end{tabular}

Characteristics of studies awaiting assessment [ordered by study ID]

\section{Aragones 2007}

Methods Study design: Cluster-randomised controlled trial

Participants Setting: Primary care

Inclusion criteria: Patients who have suffered from an episode of major depression (DSM-IV) and who have been advised to take a new course of antidepressants. Those assigned to the doctor, aged $\geq 18$ years, able to be contacted by telephone, who have been diagnosed with an episode of major depression (DSM-IV), have a score of $>14$ on the PHQ-9 (moderate-severe depression) or a score of 10 to 14 (mild depression) that has persisted for more than one month, and who have not received antidepressant medication in the previous three months

Exclusion criteria: Those who suffer from physical, psychiatric or linguistic limitations or a concurrent illness that impede comprehension/participation in the study evaluations, patients with psychotic or bipolar disorders, patients with alcohol or drug dependence and patients who are pregnant or breastfeeding

Age: Aged $\geq 18$ years 
Aragones 2007 (Continued)

Gender: Unclear

Country: Spain

Sample size: 20 primary care centres, 400 patients

Interventions Intervention: An enhanced programme for depression management. A multi-component programme with clinical, educational and organisational procedures that includes training for the health care provider and evidence-based clinical guidelines. It also includes primary care nurses working as care-managers who provide educational and emotional support for the patients and who are responsible for active and systematic clinical monitoring. The programme aims to improve the primary care/specialised level interface

Control: The doctors in the centres that continue with standard treatment use their own criteria to attend depressed patients and are allowed to use any resources they consider appropriate, including referral to the specialised level. The doctors in the control group are given a training session on diagnosing and detecting depression with the same content as that of the doctors in the intervention group

Outcomes Response and remission rates and depression severity (PHQ-9)

Notes Study complete but publication of results too late for inclusion in review at this stage; DSM-IV: Diagnostic and Statistical Manual fourth edition; PHQ-9: Patient Health Questionnaire

\section{Joubert 2006}

Methods Study design: Randomised controlled trial

Participants Setting: Hospital/primary care

Inclusion criteria: Patients aged 20 and older admitted with transient ischaemic attack or completed stroke, both first or recurrent stroke who would return to their GPs for management after discharge and who were willing to be contacted for repeat assessments over a 12 month period

Exclusion criteria: Patients discharged to nursing homes, with serious or life-threatening co-morbidities (such as cancer), non-English speaking, refused to participate, died while in hospital, cognitively impaired to the extent that they could not cooperate with follow up visits with their GP, significantly aphasic, and living more than two hours drive by car from either hospital

Age: Mean 66.45

Gender: $50 \%$ female

Country: Australia

Sample size: Total 97, intervention 46, control 51

Interventions Intervention: Integrated care programme. A shared care package was prepared for the GP. This contained goals and the recommendations for risk factor management according to clinical practice and evidence-based guidelines and recommendations for treatment of depression in stroke patients. 5 visits arranged with the GP during the 12 months post-discharge

Control: Treatment as usual. GPs contacted at 12 months for collection of study specific data

Outcomes Changes in risk factors: blood pressure, cholesterol, physical activity and depression (PHQ-9)

Notes Awaiting author response to clarify if $50 \%$ or more depressed at baseline; GP: general practitioner; PHQ-9: Patient Health Questionnaire-9 
Joubert 2008

\begin{tabular}{|c|c|}
\hline Methods & Study design: Randomised controlled trial \\
\hline Participants & $\begin{array}{l}\text { Setting: Hospital/primary care } \\
\text { Inclusion criteria: Patients aged } 20 \text { and older admitted with transient ischaemic attack or completed stroke, as } \\
\text { confirmed by CT scan } \\
\text { Exclusion criteria: Patients not returning to their GPs for management, discharged to a nursing home, serious } \\
\text { comorbidities or cognitive impairment, non-English speaking, died while in hospital, notably aphasic, lived more } \\
\text { than two hours away by car, family declining to take part, involvement in another research programme, and not } \\
\text { being assessed prior to discharge } \\
\text { Age: Mean } 65.8 \\
\text { Gender: } 45 \% \text { female } \\
\text { Country: Australia } \\
\text { Sample size: Total } 186 \text {, intervention } 91 \text {, control } 95\end{array}$ \\
\hline
\end{tabular}

Interventions Intervention: Integrated care. Patients in the integrated care group received a structured model of care that linked specialist stroke services with ongoing general practice care. GPs of treatment patients were sent an explanatory letter, as well as a comprehensive but succinct discharge summary detailing relevant investigations, risk factor profile, and medication for each patient. They also received a flowchart with goals and recommendations for risk factor management, developed from evidence-based guidelines. The study coordinator contacted each patient before and after each GP visit

Control: Standard Care patients were discharged to usual care from their GP with the standard accompanying handover information. The frequency of visits, the guidelines adopted, and the actions taken were all left up to the discretion of the GP

Outcomes Depression (PHQ-9)

Notes Awaiting author response to clarify if $50 \%$ or more depressed at baseline; CT: computer tomography; GP: general practitioner; PHQ-9: Patient Health Questionnaire-9

\section{Mareev 2010}

\begin{tabular}{ll} 
Methods & Study design: Randomised controlled trial \\
\hline Participants & $\begin{array}{l}\text { Setting: Hospital } \\
\text { Inclusion criteria: Heart failure } \\
\text { Exclusion criteria: Unclear } \\
\text { Age: Mean } 62 \text { years } \\
\text { Gender: } 39 \% \text { female } \\
\text { Country: Russia } \\
\text { Sample size: Total } 10745, \text { intervention } 5360, \text { control } 5385\end{array}$ \\
\hline Interventions & $\begin{array}{l}\text { Intervention: A multidisciplinary, non-pharmacological, intervention (including pre-discharge patient education and } \\
\text { active follow-up with regular bi-lateral telephone contact) } \\
\text { Control: Usual care }\end{array}$ \\
\hline Outcomes & Depression (HADS) \\
\hline Notes & Awaiting author response to access published or unpublished data; HADS: Hospital Anxiety and Depression Scale
\end{tabular}


Methods Study design: Randomised controlled trial

Participants Setting: Primary care

Inclusion criteria: Mild anxiety and depression

Exclusion criteria: Unclear

Age: Unclear

Gender: Unclear

Country: United Kingdom

Sample size: Total unclear, intervention unclear, control unclear

Interventions Intervention: GP based psychiatrist clinic

Control: Unclear

Outcomes Depression (HADS)

Notes National Research Register record - unable to get any further data or contact author; GP: general practitioner; HADS: Hospital Anxiety and Depression Scale

Walker 2009a

Methods Study design: Randomised controlled trial

Participants Setting: Speciality oncology clinic

Inclusion criteria: Diagnosis of cancer active within last 5 years, 18 or over, attending specialist oncology clinic, predicted survival of 12 months or more, meet DSM-IV for MDD present for 4 weeks or more

Exclusion criteria: Unable to provide consent, chronic depression (continuously depressed for 2 years or more), requires urgent psychiatric care or currently receiving same, cognitive impairment or communication difficulties, cerebral metastases, unable to attend regularly, intervention inappropriate due to medical condition,comorbid psychiatric condition or other clinical reason

Age: 18 or over

Gender: Unclear

Country: United Kingdom (Scotland)

Sample size: Total 500 planned

Interventions Intervention: Depression Care for People with Cancer

Contains the four elements of collaborative care:

1) a multi-professional approach to patient care: General practitioner (PCP), nurse (CM), SMaRT psychiatry team (MH specialist)

2) a structured management plan: Treatment phase includes a) coordination of care across professionals, b) monitoring symptoms, c) brief psychological intervention including education and PST. Maintenance phase includes completion of outcome measure and appropriate action taken via automated Interactive Voice Response or CM. MH specialist sees urgent cases or those with non-response to treatment

3) scheduled patient follow-ups: Treatment phase: 10 (30-45 minute) face-to-face sessions in 16 weeks (expected average per patient $=6-8$ ), maintenance: telephone every 4 weeks until end of trial

4) enhanced inter-professional communication: $\mathrm{CM}$ receives weekly supervision from $\mathrm{MH}$ specialist

Control: Treatment as usual enhanced as PCPs and oncologists were informed of patients diagnosis 
Walker 2009a (Continued)

$\begin{array}{ll}\text { Outcomes } & \text { Depression (HSCL-20): Treatment response at } 24 \text { weeks; remission at 12, 24, } 36 \text { and } 48 \text { weeks; severity at 12, 24, } \\ & 36 \text { and } 48 \text { weeks. Also severity of anxiety symptoms } \\ \text { QoL: } 12,24,38 \text { and } 48 \text { weeks } \\ \text { Costs: } 12,24,38,48 \text { weeks } \\ \text { Satisfaction: } 12,24,38,48 \text { weeks } \\ \text { Social: } 12,24,38,48 \text { weeks }\end{array}$

\section{Walker 2009b}

Methods Study design: Randomised controlled trial

Participants Setting: Lung cancer outpatient clinics

Inclusion criteria: Diagnosis of lung cancer, 18 or over, predicted survival of 3 months or more, meet DSM-IV for MDD present for 4 weeks or more

Exclusion criteria: Unable to provide consent, chronic depression (continuously depressed for 2 years or more), requires urgent psychiatric care or currently receiving same, cognitive impairment or communication difficulties, cerebral metastases, unable to attend regularly, intervention inappropriate due to medical condition,comorbid psychiatric condition or other clinical reason

Age: 18 or over

Gender: Unclear

Country: United Kingdom (Scotland)

Sample size: Total 200 planned

Interventions Intervention: Depression Care for People with Lung Cancer

Contains the four elements of collaborative care:

1) a multi-professional approach to patient care: General practitioner (PCP), nurse (CM), psychiatrist (MH specialist)

2) a structured management plan: Treatment phase includes a) coordination of care across professionals, b) monitoring symptoms, c) brief psychological intervention including education and PST. Maintenance phase includes completion of outcome measure and appropriate action taken by $\mathrm{CM}$. MH specialist sees urgent cases or those with non-response to treatment

3) scheduled patient follow-ups: 10 (30-45 minute) sessions in 16 weeks (expected average per patient $=6-8$ ), maintenance: telephone every 4 weeks until end of trial

4) enhanced inter-professional communication: CMs received weekly supervision from $\mathrm{MH}$ specialist

Control: Treatment as usual enhanced as PCPs and oncologists were informed of diagnosis

Outcomes Depression (HSCL-20): severity at 4, 8, 12, 16, 20, 24, 28, 32 weeks. Also severity of anxiety symptoms QoL: 4, 8, 12, 16, 20, 24, 28, 32 weeks

Costs: $4,8,12,16,20,24,28,32$ weeks

Satisfaction: $4,8,12,16,20,24,28,32$ weeks

Social: 4, 8, 12, 16, 20, 24, 28, 32 weeks

Notes

Study complete awaiting publication of results; CM: case manager; DSM-IV: Diagnostic and Statistical Manual fourth edition; HSCL: Hopkins Symptom Checklist; MDD: major depressive disorder; MH: mental health; PCP: primary care provider; PST: problem solving therapy 
Wu 2010

\begin{tabular}{|c|c|}
\hline Methods & Study design: Randomised controlled trial \\
\hline Participants & $\begin{array}{l}\text { Setting: Community psychiatry } \\
\text { Inclusion criteria: DSM-IV criteria for major depression } \\
\text { Exclusion criteria: Unclear } \\
\text { Age: Elderly } \\
\text { Gender: Unclear } \\
\text { Country: China } \\
\text { Sample size: Total } 120 \text {, intervention } 60 \text {, control } 60\end{array}$ \\
\hline Interventions & $\begin{array}{l}\text { Intervention: case management of chronic disease } \\
\text { Abstract written in English reports collaborative care provided by psychiatrists, PCP and case managers and included } \\
\text { health education, psychotherapy and antidepressants } \\
\text { Control: Treatment as usual }\end{array}$ \\
\hline Outcomes & Depression (HAMD) \\
\hline Notes & $\begin{array}{l}\text { Awaiting completion of transcription to accurately assess whether to include in review; DSM-IV: Diagnostic and } \\
\text { Statistical Manual fourth edition; PCP: primary care provider }\end{array}$ \\
\hline
\end{tabular}

\section{Characteristics of ongoing studies [ordered by study ID]}

\section{Chen 2011}

\begin{tabular}{|c|c|}
\hline Trial name or title & $\begin{array}{l}\text { Depression care management for late-life depression in China primary care: protocol for a randomised con- } \\
\text { trolled trial }\end{array}$ \\
\hline Methods & RCT \\
\hline Participants & $\begin{array}{l}\text { Patients aged } 60 \text { and over, community-dwelling residences, capable of independent communication, Mini- } \\
\text { Mental State Examination (MMSE) score } \geq 18 \text {. Major depression (PHQ-9, The Mood Disorder Module of } \\
\text { the Structured Clinical Interview for DSM-IV and the Hamilton Rating Scale for Depression) }\end{array}$ \\
\hline Interventions & $\begin{array}{l}\text { Intervention: Depression care management. Antidepressant treatment, Care managers monitor the progress } \\
\text { of treatment and side effects, educate patients/family, and facilitate communication between providers } \\
\text { Control: Care as usual. Current practice, when depression is detected by PCPs, involves suggesting to patients } \\
\text { (or family members) that they consult a mental health institution for diagnosis and treatment. There is no } \\
\text { direct referral/transfer mechanism between PCPs and mental health specialists }\end{array}$ \\
\hline Outcomes & $\begin{array}{l}\text { Depression (Hamilton Rating Scale for Depression), suicidal ideation, cognitive function, anxiety, medical } \\
\text { health, quality of life, treatment stigma, satisfaction }\end{array}$ \\
\hline Starting date & August 2010, ends July 2014 \\
\hline Contact information & Shulin Chen csl@zju.edu.cn \\
\hline
\end{tabular}




\title{
Chen 2011 (Continued)
}

\author{
Notes \\ DSM-IV: Diagnostic and Statistical Manual fourth edition; PCP: primary care provider; PHQ-9: Patient \\ Health Questionnaire-9
}

\section{Chung 2010}

\begin{tabular}{ll}
\hline Trial name or title & $\begin{array}{l}\text { Using a community partnered participatory research approach to implement a randomised controlled trial: } \\
\text { Planning community partners in care }\end{array}$ \\
\hline Methods & Randomised controlled trial \\
\hline Participants & Clients who screen as possibly having depression \\
\hline Interventions & $\begin{array}{l}\text { Compared a low-impact intervention, Resources for Services (RS), with a Community Partnered Participatory } \\
\text { Research planning process, Community Engagement and Planning (CEP), as approaches to implement } \\
\text { depression care in agencies and programs }\end{array}$ \\
\hline Outcomes & $\begin{array}{l}\text { The study assesses the impact of the different implementation approaches on community agency administrator, } \\
\text { provider and client outcomes for depression }\end{array}$ \\
\hline Starting date & 2010 \\
\hline Contact information & Bowen Chung bchung@mednet.ucla.edu \\
\hline Notes & \\
\hline
\end{tabular}

\section{Cooper 2010}

Trial name or title A cluster randomised trial of standard quality improvement versus patient-centred interventions to enhance depression care for African Americans in the primary care setting: Study protocol NCT00243425

\begin{tabular}{ll}
\hline Methods & Cluster randomised trial \\
\hline Participants & $\begin{array}{l}\text { Patients had to be between the ages of } 18 \text { and } 75 \text { years and report their race or ethnicity as African American; } \\
\text { they had to be positive on a screener for major depressive and dysthymic disorder from the CIDI. In addi- } \\
\text { tion, screen-positive patients had to meet criteria for one-year major depression on a subsequent structured } \\
\text { interview, defined as: meeting DSM-IV criteria for MDD in the past year and having symptoms present for } \\
\text { at least one week in the past month, to be considered eligible for the study }\end{array}$
\end{tabular}

Interventions Standard versus patient-centred quality improvement interventions. Both involved extensive one-on-one follow-up with a Depression Case Manager (DCM) to assess patients' depression status and to encourage adherence to recommended treatments and exposure to educational materials. The patient-centred intervention supplements standard interventions for depression by tailoring them to individual patients' stated concerns and incorporating patient-centred communication skills and cultural sensitivity training for clinicians

Outcomes

Primary outcome is change in depression symptom severity (CES-D) 


\section{Cooper 2010 (Continued)}

\begin{tabular}{ll}
\hline Starting date & March 2004, ends March 2007 \\
\hline Contact information & Lisa Cooper lisa.cooper@jhmi.edu \\
\hline Notes & $\begin{array}{l}\text { CES-D: Centre for Epidemiological Studies Depression; CIDI: Composite International Diagnostic Inter- } \\
\text { view; DSM-IV: Diagnostic and Statistical Manual fourth edition; MDD: major depressive disorder }\end{array}$ \\
\hline
\end{tabular}

Freund 2011

\begin{tabular}{ll} 
Trial name or title & $\begin{array}{l}\text { Primary care practice-based care management for chronically ill patients (PraCMan): Study protocol for a } \\
\text { cluster-randomised controlled trial }\end{array}$ \\
\hline Methods & Cluster-randomised controlled trial \\
\hline Participants & $\begin{array}{l}\text { Patients had to suffer from at least one of the following index conditions: type } 2 \text { diabetes mellitus under medical } \\
\text { treatment and/or chronic obstructive pulmonary disease under medical treatment and/or chronic heart failure } \\
\text { with confirmed diagnosis by a cardiologist. Further inclusion criteria were: High risk for future hospitalisation } \\
\text { (i.e. predicted likelihood of hospitalisation within the upper quartile of the total patient population) and age } \\
\geq 18 \text { years }\end{array}$
\end{tabular}

Interventions Intervention: PraCMan is a complex care management intervention. Based on the results of a series of exploratory studies a multifaceted intervention was developed to reduce (avoidable) hospitalisations of high risk patients. Consists of assessment, planning and monitoring

Control: Practice teams in the control group will continue to provide standard care in the context of the PCcentred care contract

Outcomes

Primary outcome: all-cause hospitalisations. Secondary outcomes: sociodemographic data, mortality, quality of life, quality of care, depression (PHQ-9), adherence, physical activity, smoking status, self-management, medication regimen, healthcare costs, activities of daily living, co-morbidity, home visits/practice visits, $\mathrm{CHF}$ decompensation (CHF patients), COPD exacerbation (COPD patients), Hypoglycaemia (DM patients) , BMI, blood pressure, fasting glucose, Hemoglobin-A1c, Dyspnoea (CHF and COPD patients), Forced expiratory volume (COPD patients)

Starting date November 2009

Contact information Tobias Freund tobias.freund@med.uni-heidelberg.de

Notes CHF: chronic heart failure; COPD: chronic obstructive pulmonary disease; PHQ-9: Patient Health Questionnaire-9; DM: diabetes mellitus

\section{Gitlin 2012}

Trial name or title A community-integrated home based depression intervention for older African Americans: description of the Beat the Blues randomised trial and intervention costs

Methods $\quad$ Randomised controlled trial 


\section{Gitlin 2012 (Continued)}

\begin{tabular}{ll}
\hline Participants & $\begin{array}{l}\text { African American, 55 years of age or older, English speaking, cognitively intact, and a score of } 5 \text { or over on } \\
\text { the PHQ-9 on two sequential testing occasions }\end{array}$ \\
\hline Interventions & $\begin{array}{l}\text { Intervention: Beat the Blues. Up to } 10 \text { one hour sessions over } 4 \text { months. Sessions integrate case management, } \\
\text { referral and linkage, depression education, stress reduction techniques and behavioural activation } \\
\text { Control: Waiting list. No study related contact following the baseline interview }\end{array}$ \\
\hline Outcomes & $\begin{array}{l}\text { Depression severity (PHQ-9). Secondary aims quality of life indicators, anxiety, depression education and } \\
\text { behavioural activation levels }\end{array}$ \\
\hline Starting date & September 2008, ends June 2012 \\
\hline Contact information & Laura Gitlin lgitlin1@jhu.edu \\
\hline Notes & PHQ-9: Patient Health Questionnaire-9 \\
\hline
\end{tabular}

\section{Graven 2011}

Trial name or title From rehabilitation to recovery: protocol for a randomised controlled trial evaluating a goal-based intervention to reduce depression and facilitate participation post-stroke

\begin{tabular}{|c|c|}
\hline Methods & Randomised controlled trial \\
\hline Participants & $\begin{array}{l}\text { All patients admitted to the inpatient hospital rehabilitation unit with the primary diagnosis of acute cere- } \\
\text { brovascular accident (inclusive of cerebral infarction, intracranial haemorrhage, and subarachnoid haemor- } \\
\text { rhage) }\end{array}$ \\
\hline Interventions & $\begin{array}{l}\text { Intervention: A multi-factorial, integrated approach which incorporates both standardised and responsive } \\
\text { components. Participants receive written material developed by the National Stroke Foundation relating to } \\
\text { recovery after the event of a stroke, written stroke information resources, including contact phone numbers } \\
\text { and websites, if available and a copy of the goals that were collaboratively devised by the participant and } \\
\text { the rehabilitation team during the final two weeks of the inpatient rehabilitation admission. Participants also } \\
\text { receive home visits and telephone contacts } \\
\text { Conrol: Participants in the control group will receive usual care as arranged by the treating team at the point } \\
\text { of discharge from the inpatient rehabilitation admission }\end{array}$ \\
\hline Outcomes & $\begin{array}{l}\text { Primary outcome is depressed mood (GDS-15). Secondary outcomes are: participation (ACS and LHS); } \\
\text { HRQoL (AQoL), activity/functional status (FIM-motor); self-efficacy (SUPPH); and cognition (MMSE) }\end{array}$ \\
\hline Starting date & March 2008 \\
\hline Contact information & Christine Graven Christine.GRAVEN@svhm.org.au; ACS: acute coronary syndrome \\
\hline Notes & $\begin{array}{l}\text { HRQoL: health related quality of life; AQoL: Assessment of Quality of Life; LHS: London Handicap Scale; } \\
\text { ACS: Activity Card Sort }\end{array}$ \\
\hline
\end{tabular}


Trial name or title Cost-effectiveness of collaborative care for chronically ill patients with comorbid depressive disorder in the general hospital setting, a randomised controlled trial

\begin{tabular}{|c|c|}
\hline Methods & Randomised controlled trial \\
\hline Participants & $\begin{array}{l}\text { All patients visiting the participating departments, who have been diagnosed with a specific chronic disease, } \\
\text { as specified in their files, will be selected. Specific diagnoses are: DM type II in the DM department, COPD } \\
\text { in the pulmonary department, and chronic heart failure or post-acute myocardial infarction in the cardio- } \\
\text { vascular department. Patients will be included in the study if they reach a cutoff score of } 15 \text { (moderate to } \\
\text { severe depressive disorder) on the PHQ-9. For patients who reach the cut-off score, the MINI-International } \\
\text { Neuropsychiatric Interview (MINI) will also be held by telephone to classify the symptoms }\end{array}$ \\
\hline Interventions & $\begin{array}{l}\text { Intervention: Based on a collaborative care model including collaboration of the patient with the treatment, } \\
\text { stepped care and collaboration between various medical disciplines. Treatment choices include antidepressant } \\
\text { medication, problem solving and manual-guided self-help } \\
\text { Control: Care as usual. Patients assigned to the care as usual group will be told that they can consult their } \\
\text { general practitioner if they feel that they need treatment, and they will be monitored }\end{array}$ \\
\hline Outcomes & $\begin{array}{l}\text { Depression severity (PHQ-9). Secondary outcome measures: cost-effectiveness, somatoform presentation, } \\
\text { associated symptoms of comorbid chronic illness, preference and adherence, life-events and social support, } \\
\text { personality traits, treatment in the care as usual group }\end{array}$ \\
\hline Starting date & 2007 \\
\hline Contact information & Eva K Horn EHorn@trimbos.nl \\
\hline Notes & $\begin{array}{l}\text { COPD: chronic obstructive pulmonary disease; PHQ-9: Patient Health Questionnaire-9; DM: diabetes } \\
\text { mellitus }\end{array}$ \\
\hline
\end{tabular}

\section{Ijff 2007}

Trial name or title Cost-effectiveness of collaborative care including PST and an antidepressant treatment algorithm for the treatment of major depressive disorder in primary care; a randomised clinical trial

\begin{tabular}{ll}
\hline Methods & Randomised controlled trial \\
\hline Participants & $\begin{array}{l}\text { Patients with a diagnosis of major depressive disorder and who have dysfunction due to this depressive disorder } \\
\text { (i.e. by the loss of role-functioning in daily life). Patients will be included if they reach the cut-off score of } 15 \\
\text { on the PHQ-9 }\end{array}$
\end{tabular}

Interventions Intervention: Collaborative care. A treatment plan is jointly formulated by the care manager, the patient and the GP together Treatment choices include PST and antidepressant medication

Control: Care as usual

Outcomes

Severity of depression symptoms (PHQ-9). Secondary outcome measures: remission of depression symptoms, cost-utility and physical comorbidity

Starting date

2007 
Ijff 2007 (Continued)

Contact information Marjoliek A IJff mijff@trimbos.nl

Notes GP: general practitioner; PHQ-9: Patient Health Questionnaire-9; PST: problem solving therapy

Mitchell 2011

Trial name or title A randomised evaluation of collaborative care and active-surveillance for screen-positive elders with subthreshold depression (CASPER): study protocol for a randomised controlled trial

\begin{tabular}{ll}
\hline Methods & Randomised controlled trial \\
\hline Participants & $\begin{array}{l}\text { People aged over } 75 \text { years with sub-threshold depression assessed using The major depressive episode module } \\
\text { of the Mini International Neuropsychiatric Interview (MINI) }\end{array}$
\end{tabular}

Interventions Intervention: Collaborative care with behavioural activation plus usual GP care intervention: Low intensity collaborative care which has been designed specifically for those aged 75 or over with subthreshold depression, over 8-10 weekly sessions. The defining features of collaborative care include a case manager working with the participant, with access to the GP and a mental health specialist Control: Usual GP care

Outcomes

Depression severity and symptomatology at four months (PHQ-9). Secondary outcomes: depression severity and symptomatology (at 12 months), binary description of the PHQ-9 (at 4 and 12 months), quality of life measures (at 4 and 12 months), psychological anxiety (at 4 and 12 months), medication (at 4 and 12 months) , and mortality (at 4 and 12 months)

Starting date October 2009, end June 2013

Contact information Simon Gilbody simon.gilbody@york.ac.uk

Notes $\quad$ GP: general practitioner; PHQ-9: Patient Health Questionnaire-9

Morgan 2009

Trial name or title The TrueBlue study: Is practice nurse-led collaborative care effective in the management of depression for patients with heart disease or diabetes?

Methods Cluster-randomised intervention trial

Participants Patients with a diagnosis of CHD or T2 DM, patients who are either under 18 years of age or in residential care are excluded from the study. Presence of at least mild depression assessed by PHQ-9 of greater than 5

Interventions

Intervention: Nurse-led collaborative care. Patients will be invited to attend a practice nurse consultation every 3 months prior to seeing their usual general practitioner. The PN will assess psychological, physiological and lifestyle parameters then work with the patient to set management goals. The outcome of this assessment will form the basis of a GP Management Plan document

Control: Patients will continue to receive their usual care for the first six months of the study before the PNs undergo the training and switch to the intervention protocol 


\section{Morgan 2009 (Continued)}

\begin{tabular}{ll}
\hline Outcomes & Depression (PHQ-9) \\
\hline Starting date & 2009 \\
\hline Contact information & Mark Morgan mark.morgan@greaterhealth.org \\
\hline Notes & $\begin{array}{l}\text { CHD: coronary heart disease; GP: general practitioner; PHQ-9: Patient Health Questionnaire-9; DM: dia- } \\
\text { betes mellitus }\end{array}$ \\
\hline
\end{tabular}

Muntingh 2009

\begin{tabular}{ll} 
Trial name or title & $\begin{array}{l}\text { Collaborative stepped care for anxiety disorders in primary care: aims and design of a randomised controlled } \\
\text { trial }\end{array}$ \\
\hline Methods & Randomised controlled trial \\
\hline Participants & $\begin{array}{l}\text { Patients with a primary diagnosis of PD with or without agoraphobia and/or a primary diagnosis of GAD } \\
\text { according to the criteria of the DSM-IV will be included in the study. Patients who are suicidal, suffer from } \\
\text { dementia or other severe cognitive disorders, psychotic disorder, bipolar disorder, dependence on drugs or } \\
\text { alcohol, or with an unstable severe medical condition as diagnosed by their GP or as assessed in a diagnostic } \\
\text { interview will be excluded. Patients with insufficient knowledge of the Dutch language to fill out the ques- } \\
\text { tionnaires, patients who are already receiving intensive psychological treatment (> } 2 \text { contacts per month with } \\
\text { a psychologist or psychiatrist) and patients who are under } 18 \text { years of age will also be excluded from the study }\end{array}$ \\
\hline $\begin{array}{l}\text { Intervention: Collaborative stepped-care. Care is provided by a team of the GP, the care manager, the patient } \\
\text { and a consultant psychiatrist. The collaborative stepped care intervention is composed of four steps: guided } \\
\text { self-help, CBT, antidepressants, optimisation of medication in primary care or referral to secondary care } \\
\text { Control: Care as usual comprises every form of care the GP is used to offer to his patient (e.g. watchful } \\
\text { waiting, prescription of medication, referral to a mental health care professional or any other form of care the } \\
\text { GP offers to his patient) }\end{array}$
\end{tabular}

Outcomes Anxiety severity (Beck anxiety inventory). Secondary outcome measure: remission. Other outcome measures: anxiety severity and impairment, physical symptoms, quality of life

\begin{tabular}{ll}
\hline Starting date & 2009 \\
\hline Contact information & Anna DT Muntingh amuntingh@trimbos.nl \\
\hline Notes & $\begin{array}{l}\text { CBT: cognitive behaviour therapy; DSM-IV: Diagnostic and Statistical Manual fourth edition; GAD: gen- } \\
\text { eralised anxiety disorder; GP: general practitioner; PD: panic disorder }\end{array}$ \\
\hline
\end{tabular}


Musselman 2006

Trial name or title Depression-Diabetes Mechanisms: Urban African Americans

\begin{tabular}{ll}
\hline Methods & Randomised controlled trial \\
\hline Participants & $\begin{array}{l}\text { Subjects must be English-speaking, African American, have type 2 diabetes per American Diabetes Association } \\
\text { criteria, patient's receiving care at Grady Hospital }\end{array}$ \\
\hline Interventions & $\begin{array}{l}\text { Intervention: Computer-based cognitive behavioral therapy (CBT) programme entitled "Beating the Blues" } \\
+ \text { the SSRI antidepressant escitalopram } \\
\text { Control: computer-based cognitive behavioral therapy (CBT) programme entitled "Beating the Blues“ + } \\
\text { placebo }\end{array}$ \\
\hline Outcomes & $\begin{array}{l}\text { Primary outcomes: Glycemic control: assessed as levels of HbAlc, neurometabolic variables, adherence, } \\
\text { variability in follow-up }\end{array}$ \\
\hline Starting date & May 2004, end May 2008 \\
\hline Contact information & Dominique L Musselman \\
\hline Notes & SSRI: selective serotonin reuptake inhibitor \\
\hline
\end{tabular}

Pommer 2012

Trial name or title Managing comorbid depression and anxiety in primary care patients with asthma and/or chronic obstructive pulmonary disease: study protocol for a randomised controlled trial

\begin{tabular}{|c|c|}
\hline Methods & Randomised controlled trial \\
\hline Participants & $\begin{array}{l}\text { Patients included in the AsCoZoB management programme for patients with asthma/COPD are eligible. } \\
\text { Exclusion criteria - aged below } 18 \text {, currently receiving treatment for depression and/or anxiety, diagnosed } \\
\text { with a psychiatric disorder, suicidal ideation, not being able to read or speak Dutch sufficiently. Depression } \\
\text { and anxiety assessed using the Mini International Neuropsychiatric Interview (MINI) }\end{array}$ \\
\hline Interventions & $\begin{array}{l}\text { Intervention: Disease management condition - stepped care programme consisting of three consecutive steps } \\
\text { and monitoring of results. Stepped care intervention includes psychoeducation, course on coping with de- } \\
\text { pression/anxiety and coaching complemented with antidepressant/anxiolytic medication } \\
\text { Control: Care as usual }\end{array}$ \\
\hline Outcomes & Primary outcome measures: Depression and anxiety (PHQ-9, GAD-7, MINI) and quality of life/health status \\
\hline Starting date & January 2011, end December 2013 \\
\hline Contact information & Francois Pouwer F.Pouwer@uvt.nl \\
\hline Notes & $\begin{array}{l}\text { COPD: chronic obstructive pulmonary disease; GAD: generalised anxiety disorder; PHQ-9: Patient Health } \\
\text { Questionnaire-9 }\end{array}$ \\
\hline
\end{tabular}


Rollman 2004

Trial name or title Telephone-based care management programme for individuals with anxiety disorders

\begin{tabular}{ll}
\hline Methods & RCT \\
\hline Participants & $\begin{array}{l}\text { 18-64 years, diagnosis of panic disorder or generalised anxiety disorder, score of } 7 \text { or higher on the Panic } \\
\text { Disorder Severity Scale OR a score of } 14 \text { or higher on the Structured Interview Guide for the Hamilton } \\
\text { Anxiety Scale, life expectancy greater than 1 year, has household telephone, able to read and write in English }\end{array}$ \\
\hline Interventions & $\begin{array}{l}\text { Intervention: Telephone based collaborative care } \\
\text { Usual care: Treatment as usual }\end{array}$ \\
\hline Outcomes & $\begin{array}{l}\text { Primary: Health-related quality of life (SF-36 MCS) at 12 months } \\
\text { Secondary: Clinical (anxiety and depression), alcohol use, health services utilisation, health care costs - all at } \\
\text { 12 months }\end{array}$ \\
\hline Starting date & March 2004 ends December 2012 \\
\hline Contact information & Bruce Rollman \\
\hline Notes & \\
\hline
\end{tabular}

Steel 2011

\begin{tabular}{|c|c|}
\hline Trial name or title & $\begin{array}{l}\text { Randomised controlled trial of a collaborative care intervention to manage cancer-related symptoms: lessons } \\
\text { learned }\end{array}$ \\
\hline Methods & Randomised controlled trial \\
\hline Participants & $\begin{array}{l}\text { Inclusion criteria: Biopsy, radiological, and/or biological evidence of hepatobiliary carcinoma; age } 18 \text { years or } \\
\text { older; and fluency in English. Exclusion criteria included: current suicidal or homicidal ideation, or current } \\
\text { psychosis, or thought disorder }\end{array}$ \\
\hline Interventions & $\begin{array}{l}\text { Intervention: Collaborative care intervention. Combination of both cognitive-behavioral and pharmacological } \\
\text { treatment. The delivery of the intervention included face-to-face visits whenever the patient came into the } \\
\text { outpatient clinic or hospital for cancer treatment, telephone follow-up with a minimum of two telephone } \\
\text { contacts (before and after the patients' cancer treatment) between cancer treatments, and access to a website } \\
\text { that was designed specifically for this RCT, which includes educational information, a self-management area, } \\
\text { journaling, a chat room, an audiovisual library, peer support, and other resources } \\
\text { Control: Enhanced usual care }\end{array}$ \\
\hline Outcomes & $\begin{array}{l}\text { Primary outcomes: Depression (CES-D), pain, fatigue, quality of life. Secondary outcomes: Anxiety, sleep } \\
\text { quality, sexual functioning, substance use, healthcare utilisation and satisfaction with healthcare }\end{array}$ \\
\hline Starting date & 2011 \\
\hline Contact information & Jennifer L Steel steeljl@upmc.edu \\
\hline Notes & CES-D: Centre for Epidemiological Studies Depression Scale; RCT: randomised controlled trial \\
\hline
\end{tabular}




\begin{tabular}{ll} 
Trial name or title & $\begin{array}{l}\text { Disease management for comorbid depression and anxiety in diabetes mellitus: design of a randomised } \\
\text { controlled trial in primary care }\end{array}$ \\
\hline Methods & Randomised controlled trial \\
\hline Participants & $\begin{array}{l}\text { Type } 2 \text { diabetes mellitus patients, aged } 18 \text { or over and with elevated depressive (PHQ-9 score } 7 \text { or more) } \\
\text { and/or anxiety symptoms (GAD-7 score } 8 \text { or more). Patients are excluded if they currently receive psycho- } \\
\text { logical treatment for their symptoms of depression or anxiety, experience major psychiatric problems, such as } \\
\text { schizophrenia and suicidal ideation, are addicted to alcohol, drugs or gambling, are cognitively impaired, or } \\
\text { are unable to read or speak Dutch sufficiently }\end{array}$
\end{tabular}

Interventions Intervention: DiMaCoDeA-DM2 (disease management intervention for comorbid depression and anxiety in patients with DM2) - active screening, stepped care treatment and monitoring of depression/anxiety

Control: Care as usual

Outcomes Depression and anxiety (PHQ-9 and GAD-7). Secondary outcomes: Quality of life, health status, diabetes specific distress, self-management, medication adherence and cost-effectiveness

Starting date January 2011, end December 2013

Contact information Francois Pouwer f.pouwer@uvt.nl

Notes GAD: generalised anxiety disorder; PHQ-9: Patient Health Questionnaire-9; DM: diabetes mellitus

\section{Trinh 2011}

Trial name or title A study of a culturally focused psychiatric consultation service for Asian American and Latino American primary care patients with depression

\begin{tabular}{ll}
\hline Methods & Randomised controlled trial \\
\hline Participants & $\begin{array}{l}\text { Adults who are } 18 \text { years of age or older; are members of either targeted Asian American or Latino American } \\
\text { minority groups; screen positive for symptoms of depression; and are able to consent to study participation. } \\
\text { Patients will be excluded if they have active unstable, untreated psychiatric illness precluding participation in } \\
\text { the study (e.g., actively suicidal or homicidal or actively psychotic). Patients in the intervention arm will be } \\
\text { excluded if they have bipolar disorder }\end{array}$
\end{tabular}

Interventions Intervention: Culturally focused psychiatric intervention (CFP). Patients receiving the intervention will undergo a CFP consultation assessment and will receive a culturally appropriate CFP consultation patient toolkit, available in their language of preference (i.e. English, Spanish, Chinese, or Vietnamese), as well as training in using the toolkit materials

Control: Usual care

Outcomes Primary outcomes will determine the feasibility and cost associated with implementation of the service, and evaluate patient and provider satisfaction with the CFP service

Starting date $\quad$ December 2009, end August 2011 
Trinh 2011 (Continued)

Contact information Nhi-Ha T Trinh ntrinh@partners.org

Notes

Tylee 2011

Trial name or title Up-Beat UK: A programme of research into the relationship between coronary heart disease and depression in primary care patients

\begin{tabular}{ll} 
Methods & Study design: Pilot randomised controlled trial \\
\hline Participants & $\begin{array}{l}\text { Patients aged } 16 \text { or older, scoring } 3 \text { or more on the PHQ-2, and with symptomatic CHD will then be assessed } \\
\text { further using the Hospital Anxiety and Depression Scale (HADS). If they score }>9 \text { on the depression scale } \\
\text { of HADS they will be eligible to participate in the study }\end{array}$ \\
$\begin{array}{l}\text { Exclusion criteria: Temporary registrations, actively suicidal patients, psychotic depression as evidenced by } \\
\text { delusions and/or hallucinations, non-English speaking, participants currently in hospital for treatment of } \\
\text { their CHD }\end{array}$
\end{tabular}

Interventions Intervention: The nature of the intervention will be determined by the results of two qualitative studies Control: Treatment as usual by GP and any other relevant professionals

Outcomes

Primary outcome depression (Hospital Anxiety and Depression Scale). Secondary outcomes depression, CHD, quality of life, adherence to medication, life events, social problems, health service utilisation, illness perceptions, well-being, and participants problem priorities

Starting date Protocol published 2011

Contact information a.tylee@iop.kcl.ac.uk

Notes CHD: coronary heart disease; HADS: Hospital Anxiety and Depression Scale

\section{Yeung 2011}

Trial name or title A study of the effectiveness of telepsychiatry-based culturally sensitive collaborative treatment of depressed Chinese Americans

\begin{tabular}{ll}
\hline Methods $\quad$ Randomised controlled trial \\
\hline
\end{tabular}

Participants Patients will be included if they are monolingual Chinese Americans, meaning that they require or prefer to be interviewed in Chinese (Cantonese or Mandarin), are 18 years of age or older, are competent to consent to study participation, meet criteria for MDD as diagnosed by the Mini International Neuropsychiatric Interview (MINI), receive a score of 10 or greater on the CB-PHQ-9, and are willing to participate in phone interviews for symptom monitoring, as well as for care management if they are randomised to the treatment group

Interventions

Intervention: Telepsychiatry-based Culturally Sensitive Collaborative Treatment (T-CSCT) from a multidisciplinary team involving assessment and care management to

monitor patients' psychiatric treatment as well as to consolidate and streamline the treatment efforts of the 


\section{Yeung 2011 (Continued)}

patient's PCP and psychiatrist

Control: Usual care

Outcomes $\quad$ Outcome measures include depressive symptom severity (HAM-D) as well as patient and PCP satisfaction with the telepsychiatry-based care management service

Starting date January 2009, end July 2014

Contact information Albert Yeung ayeung@partners.org

Notes

HAM-D: Hamilton Depression Rating Scale; MDD: major depressive disorder; PCP: primary care provider

\section{Zatzick 2011}

Trial name or title Enhancing the population impact of collaborative care interventions: mixed method development and implementation of stepped care targeting post-traumatic stress disorder and related comorbidities after acute trauma

\begin{tabular}{ll}
\hline Methods & Randomised controlled trial \\
\hline Participants & $\begin{array}{l}\text { English-speaking women and men } 18 \text { years and older who presented to Harborview with injuries so severe } \\
\text { that they required inpatient surgical admissions. Patients who had suffered head, spinal cord or other severe } \\
\text { injuries that prevented participation in the inpatient ward interview were excluded from the study. Patients } \\
\text { who required immediate intervention (i.e. self-inflicted injury, active psychosis) were referred for evaluation to } \\
\text { the inpatient psychiatric consult service. Patients who were currently incarcerated or who had recent histories } \\
\text { of severe violence were also excluded. Patients living at great distances from the trauma centre (i.e. N50- } \\
100 \text { miles) were excluded as the investigative team anticipated difficulty in long distance care co-ordination } \\
\text { activities }\end{array}$ \\
\hline
\end{tabular}

Interventions Intervention: Stepped collaborative care intervention. The intervention included trauma-focused care management, and an initial choice of starting with either evidence-based pharmacotherapy or CBT targeting PTSD and related comorbidities

Control: Usual care

Outcomes

PTSD symptom reduction (PTSD checklist (PCL) and the Clinician-Administered PTSD Scale (CAPS)).

Secondary outcomes include ratings of substance use and functional outcomes

Starting date

Contact information Douglas Ztzick dzatzick@u.washington.edu

Notes

CBT: cognitive behaviour therapy; PTSD: post-traumatic stress disorder 
DATA ANDANALYSES

Comparison 1. Collaborative care versus 'usual care' (adults)

\begin{tabular}{|c|c|c|c|c|}
\hline Outcome or subgroup title & $\begin{array}{l}\text { No. of } \\
\text { studies }\end{array}$ & $\begin{array}{c}\text { No. of } \\
\text { participants }\end{array}$ & Statistical method & Effect size \\
\hline $\begin{array}{l}1 \text { Improvement in depression } \\
\text { symptoms }\end{array}$ & 33 & & Std. Mean Difference (IV, Random, 95\% CI) & Subtotals only \\
\hline 1.10 to 6 months & 30 & 5984 & Std. Mean Difference (IV, Random, 95\% CI) & $-0.34[-0.41,-0.27]$ \\
\hline 1.27 to 12 months & 13 & 4092 & Std. Mean Difference (IV, Random, 95\% CI) & $-0.28[-0.41,-0.15]$ \\
\hline 1.313 to 24 months & 1 & 1379 & Std. Mean Difference (IV, Random, 95\% CI) & $-0.35[-0.46,-0.24]$ \\
\hline $\begin{array}{l}1.40 \text { to } 6 \text { months (cluster } \\
\text { ICC } 0.00 \text { ) }\end{array}$ & 30 & 6786 & Std. Mean Difference (IV, Random, 95\% CI) & $-0.33[-0.39,-0.26]$ \\
\hline $\begin{array}{l}1.50 \text { to } 6 \text { months (cluster } \\
\text { ICC } 0.05 \text { ) }\end{array}$ & 30 & 5946 & Std. Mean Difference (IV, Random, 95\% CI) & $-0.34[-0.41,-0.26]$ \\
\hline $\begin{array}{l}1.60 \text { to } 6 \text { months (sensitivity } \\
\text { analysis - cluster comparisons } \\
\text { removed) }\end{array}$ & 21 & 4361 & Std. Mean Difference (IV, Random, 95\% CI) & $-0.37[-0.46,-0.28]$ \\
\hline $\begin{array}{l}1.70 \text { to } 6 \text { months (sensitivity } \\
\text { analysis - comparisons } \\
\text { including patients with physical } \\
\text { comorbidity removed) }\end{array}$ & 23 & 5082 & Std. Mean Difference (IV, Random, 95\% CI) & $-0.29[-0.37,-0.21]$ \\
\hline $\begin{array}{l}1.80 \text { to } 6 \text { months (sensitivity } \\
\text { analysis - comparisons at risk } \\
\text { of bias due to allocation of } \\
\text { concealment removed) }\end{array}$ & 14 & 3758 & Std. Mean Difference (IV, Random, 95\% CI) & $-0.34[-0.42,-0.26]$ \\
\hline $\begin{array}{l}1.90 \text { to } 6 \text { months (sensitivity } \\
\text { analysis - comparisons at risk } \\
\text { of bias due to loss to follow-up } \\
\text { removed) }\end{array}$ & 27 & 5793 & Std. Mean Difference (IV, Random, 95\% CI) & $-0.33[-0.40,-0.26]$ \\
\hline $\begin{array}{l}1.107 \text { to } 12 \text { months } \\
\text { (sensitivity analysis - } \\
\text { comparisons with intervention } \\
\text { length }>6 \text { months removed) }\end{array}$ & 6 & 1300 & Std. Mean Difference (IV, Random, 95\% CI) & $-0.19[-0.30,-0.08]$ \\
\hline 2 Depression response & 58 & & Risk Ratio (M-H, Random, 95\% CI) & Subtotals only \\
\hline 2.10 to 6 months & 48 & 11250 & Risk Ratio (M-H, Random, 95\% CI) & $1.32[1.22,1.43]$ \\
\hline 2.27 to 12 months & 29 & 8001 & Risk Ratio (M-H, Random, 95\% CI) & $1.31[1.17,1.48]$ \\
\hline 2.313 to 24 months & 6 & 2983 & Risk Ratio (M-H, Random, 95\% CI) & $1.29[1.18,1.41]$ \\
\hline $2.425+$ months & 5 & 943 & Risk Ratio (M-H, Random, 95\% CI) & $1.12[0.98,1.27]$ \\
\hline $\begin{array}{l}2.50 \text { to } 6 \text { months (cluster } \\
\text { ICC } 0.00 \text { ) }\end{array}$ & 48 & 13459 & Risk Ratio (M-H, Random, 95\% CI) & $1.32[1.22,1.42]$ \\
\hline $\begin{array}{l}2.60 \text { to } 6 \text { months (cluster } \\
\text { ICC } 0.05 \text { ) }\end{array}$ & 48 & 10346 & Risk Ratio (M-H, Random, 95\% CI) & $1.34[1.23,1.45]$ \\
\hline $\begin{array}{l}2.70 \text { to } 6 \text { months (sensitivity } \\
\text { analysis - cluster comparisons } \\
\text { removed) }\end{array}$ & 39 & 8500 & Risk Ratio (M-H, Random, 95\% CI) & $1.35[1.22,1.49]$ \\
\hline
\end{tabular}

Collaborative care for depression and anxiety problems (Review)

Copyright $\odot 2012$ The Cochrane Collaboration. Published by John Wiley \& Sons, Ltd. 
2.80 to 6 months (sensitivity analysis - comparisons including patients with physical comorbidity removed)

2.90 to 6 months (sensitivity analysis - comparisons at risk of bias due to allocation of concealment removed)

2.100 to 6 months (sensitivity analysis - comparisons at risk of bias due to loss to follow-up removed)

2.117 to 12 months (sensitivity analysis comparisons with intervention length > 6 months removed)

3 Antidepressant medication use 3.10 to 6 months 3.27 to 12 months 3.313 to 24 months $3.425+$ months

4 Improvement in anxiety symptoms

4.10 to 6 months 4.27 to 12 months 4.313 to 24 months

5 Anxiety response 5.10 to 6 months 5.27 to 12 months 5.313 to 24 months

6 Anxiety medication use 6.10 to 6 months 6.27 to 12 months 6.313 to 24 months

7 Mental QoL

7.10 to 6 months

7.27 to 12 months

7.313 to 24 months

$7.425+$ months

8 Physical QoL

8.10 to 6 months

8.27 to 12 months

8.313 to 24 months

9 Patient satisfaction

10 Patient satisfaction
Risk Ratio (M-H, Random, 95\% CI)

Risk Ratio (M-H, Random, 95\% CI)

Risk Ratio (M-H, Random, 95\% CI)

Risk Ratio (M-H, Random, 95\% CI)

Risk Ratio (M-H, Random, 95\% CI)

Std. Mean Difference (IV, Random, 95\% CI)

Std. Mean Difference (IV, Random, 95\% CI) Std. Mean Difference (IV, Random, 95\% CI) Std. Mean Difference (IV, Random, 95\% CI) Risk Ratio (M-H, Random, 95\% CI) Risk Ratio (M-H, Random, 95\% CI) Risk Ratio (M-H, Random, 95\% CI) Risk Ratio (M-H, Random, 95\% CI) Risk Ratio (M-H, Random, 95\% CI) Risk Ratio (M-H, Random, 95\% CI) Risk Ratio (M-H, Random, 95\% CI) Risk Ratio (M-H, Random, 95\% CI) Std. Mean Difference (IV, Random, 95\% CI) Std. Mean Difference (IV, Random, 95\% CI) Std. Mean Difference (IV, Random, 95\% CI) Std. Mean Difference (IV, Random, 95\% CI) Std. Mean Difference (IV, Random, 95\% CI) Std. Mean Difference (IV, Random, 95\% CI) Std. Mean Difference (IV, Random, 95\% CI) Std. Mean Difference (IV, Random, 95\% CI) Std. Mean Difference (IV, Random, 95\% CI) Std. Mean Difference (IV, Random, 95\% CI) Risk Ratio (M-H, Random, 95\% CI)
Subtotals only $1.47[1.33,1.63]$

$1.43[1.26,1.61]$

$1.22[1.03,1.45]$

$1.02[0.87,1.21]$

Totals not selected

$0.0[0.0,0.0]$

$0.0[0.0,0.0]$

$0.0[0.0,0.0]$

Subtotals only

1.50 [1.21, 1.87]

$1.41[1.18,1.69]$

1.26 [1.11, 1.42]

Subtotals only

$1.24[0.93,1.63]$

1.17 [1.03, 1.32]

1.09 [0.92, 1.30]

Subtotals only $0.26[0.13,0.38]$ 0.20 [0.09, 0.31] 0.25 [0.08, 0.43] $0.10[-0.03,0.23]$ Subtotals only $0.06[-0.01,0.13]$ $0.07[-0.04,0.18]$ $0.10[0.02,0.17]$ $0.31[0.13,0.49]$ $1.27[1.18,1.38]$ 


\begin{tabular}{|c|c|c|c|c|}
\hline Outcome or subgroup title & $\begin{array}{l}\text { No. of } \\
\text { studies }\end{array}$ & $\begin{array}{c}\text { No. of } \\
\text { participants }\end{array}$ & Statistical method & Effect size \\
\hline 1 Depression symptoms & 2 & & Std. Mean Difference (IV, Random, 95\% CI) & Subtotals only \\
\hline 1.10 to 6 months & 2 & 471 & Std. Mean Difference (IV, Random, 95\% CI) & $-0.17[-0.35,0.01]$ \\
\hline 1.27 to 12 months & 1 & 114 & Std. Mean Difference (IV, Random, 95\% CI) & $-0.32[-0.69,0.05]$ \\
\hline 2 Depression & 2 & & Risk Ratio (M-H, Random, 95\% CI) & Subtotals only \\
\hline 2.10 to 6 months & 2 & 460 & Risk Ratio (M-H, Random, 95\% CI) & $0.73[0.56,0.96]$ \\
\hline 2.27 to 12 months & 2 & 441 & Risk Ratio (M-H, Random, 95\% CI) & $1.05[0.54,2.06]$ \\
\hline 2.313 to 24 months & 1 & 322 & Risk Ratio (M-H, Random, 95\% CI) & $0.75[0.51,1.11]$ \\
\hline 3 Antidepressant medication use & 1 & & Risk Ratio (M-H, Random, 95\% CI) & Totals not selected \\
\hline 3.10 to 6 months & 1 & & Risk Ratio (M-H, Random, 95\% CI) & $0.0[0.0,0.0]$ \\
\hline 3.27 to 12 months & 1 & & Risk Ratio (M-H, Random, 95\% CI) & $0.0[0.0,0.0]$ \\
\hline 3.313 to 24 months & 1 & & Risk Ratio (M-H, Random, 95\% CI) & $0.0[0.0,0.0]$ \\
\hline 4 Mental QoL & 2 & & Std. Mean Difference (IV, Random, 95\% CI) & Subtotals only \\
\hline 4.10 to 6 months & 2 & 471 & Std. Mean Difference (IV, Random, 95\% CI) & $0.15[-0.03,0.33]$ \\
\hline 4.27 to 12 months & 2 & 441 & Std. Mean Difference (IV, Random, 95\% CI) & $0.05[-0.24,0.33]$ \\
\hline 4.313 to 24 months & 1 & 322 & Std. Mean Difference (IV, Random, 95\% CI) & $0.09[-0.13,0.31]$ \\
\hline 5 Physical QoL & 1 & & Std. Mean Difference (IV, Random, 95\% CI) & Totals not selected \\
\hline 5.10 to 6 months & 1 & & Std. Mean Difference (IV, Random, 95\% CI) & $0.0[0.0,0.0]$ \\
\hline 5.27 to 12 months & 1 & & Std. Mean Difference (IV, Random, 95\% CI) & $0.0[0.0,0.0]$ \\
\hline 6 Patient satisfaction & 2 & 471 & Std. Mean Difference (IV, Random, 95\% CI) & $0.09[-0.38,0.57]$ \\
\hline
\end{tabular}

Comparison 3. Collaborative care versus feedback (adults)

\begin{tabular}{lcccc} 
Outcome or subgroup title & $\begin{array}{c}\text { No. of } \\
\text { studies }\end{array}$ & $\begin{array}{c}\text { No. of } \\
\text { participants }\end{array}$ & Statistical method & Effect size \\
\hline 1 Depression & 1 & Risk Ratio (M-H, Random, 95\% CI) & Totals not selected \\
1.10 to 6 months & 1 & Risk Ratio (M-H, Random, 95\% CI) & $0.0[0.0,0.0]$ \\
\hline
\end{tabular}

\section{Comparison 4. Collaborative care versus consultation liaison (adults)}

\begin{tabular}{|c|c|c|c|c|}
\hline Outcome or subgroup title & $\begin{array}{l}\text { No. of } \\
\text { studies }\end{array}$ & $\begin{array}{c}\text { No. of } \\
\text { participants }\end{array}$ & Statistical method & Effect size \\
\hline 1 Depression & 1 & & Risk Ratio (M-H, Random, 95\% CI) & Totals not selected \\
\hline 1.10 to 6 months & 1 & & Risk Ratio (M-H, Random, 95\% CI) & $0.0[0.0,0.0]$ \\
\hline 1.27 to 12 months & 1 & & Risk Ratio (M-H, Random, 95\% CI) & $0.0[0.0,0.0]$ \\
\hline
\end{tabular}


Comparison 5. Collaborative care plus consultation liaison versus collaborative care (adults)

\begin{tabular}{|c|c|c|c|c|}
\hline Outcome or subgroup title & $\begin{array}{l}\text { No. of } \\
\text { studies }\end{array}$ & $\begin{array}{c}\text { No. of } \\
\text { participants }\end{array}$ & Statistical method & Effect size \\
\hline 1 Depression & 1 & & Risk Ratio (M-H, Random, 95\% CI) & Totals not selected \\
\hline 1.10 to 6 months & 1 & & Risk Ratio (M-H, Random, 95\% CI) & $0.0[0.0,0.0]$ \\
\hline $1.225+$ months & 1 & & Risk Ratio (M-H, Random, 95\% CI) & $0.0[0.0,0.0]$ \\
\hline
\end{tabular}

\section{Comparison 6. Collaborative care versus enhanced referral (adults)}

\begin{tabular}{lcccc} 
Outcome or subgroup title & $\begin{array}{c}\text { No. of } \\
\text { studies }\end{array}$ & $\begin{array}{c}\text { No. of } \\
\text { participants }\end{array}$ & Statistical method & Effect size \\
\hline 1 Depression symptoms & 1 & Std. Mean Difference (IV, Random, 95\% CI) & Totals not selected \\
1.10 to 6 months & 1 & Std. Mean Difference (IV, Random, 95\% CI) & $0.0[0.0,0.0]$ \\
\hline
\end{tabular}

Comparison 7. Collaborative care (psychotherapy) versus collaborative care (medication) (adults)

\begin{tabular}{|c|c|c|c|c|}
\hline Outcome or subgroup title & $\begin{array}{l}\text { No. of } \\
\text { studies }\end{array}$ & $\begin{array}{c}\text { No. of } \\
\text { participants }\end{array}$ & Statistical method & Effect size \\
\hline 1 Depression & 1 & & Risk Ratio (M-H, Random, 95\% CI) & Totals not selected \\
\hline 1.10 to 6 months & 1 & & Risk Ratio (M-H, Random, 95\% CI) & $0.0[0.0,0.0]$ \\
\hline 1.27 to 12 months & 1 & & Risk Ratio (M-H, Random, 95\% CI) & $0.0[0.0,0.0]$ \\
\hline 1.313 to 24 months & 1 & & Risk Ratio (M-H, Random, 95\% CI) & $0.0[0.0,0.0]$ \\
\hline $1.425+$ months & 1 & & Risk Ratio (M-H, Random, 95\% CI) & $0.0[0.0,0.0]$ \\
\hline
\end{tabular}

Comparison 8. Collaborative care plus psychotherapy versus collaborative care (adults)

\begin{tabular}{|c|c|c|c|c|}
\hline Outcome or subgroup title & $\begin{array}{l}\text { No. of } \\
\text { studies }\end{array}$ & $\begin{array}{c}\text { No. of } \\
\text { participants }\end{array}$ & Statistical method & Effect size \\
\hline 1 Depression symptoms & 1 & & Std. Mean Difference (IV, Random, 95\% CI) & Totals not selected \\
\hline 1.10 to 6 months & 1 & & Std. Mean Difference (IV, Random, 95\% CI) & $0.0[0.0,0.0]$ \\
\hline 2 Depression & 3 & & Risk Ratio (M-H, Random, 95\% CI) & Subtotals only \\
\hline 2.10 to 6 months & 2 & 488 & Risk Ratio (M-H, Random, 95\% CI) & $1.14[0.97,1.33]$ \\
\hline 2.27 to 12 months & 1 & 41 & Risk Ratio (M-H, Random, 95\% CI) & $1.17[0.79,1.75]$ \\
\hline $2.325+$ months & 1 & 137 & Risk Ratio (M-H, Random, 95\% CI) & $1.43[0.90,2.26]$ \\
\hline
\end{tabular}




\section{H I S T O R Y}

Protocol first published: Issue 2, 2007

Review first published: Issue 10, 2012

\begin{tabular}{l|ll}
\hline Date & Event & Description \\
\hline 27 February 2012 & Amended & Methodology updated \\
\hline 21 April 2010 & Amended & $\begin{array}{l}\text { Changed contact details of first/contact author (who has changed surname from Fletcher to Archer) } \\
\text {; date of 'Next stage expected' altered; some references corrected }\end{array}$ \\
\hline 29 October 2008 & Amended & Converted to new review format. \\
\hline
\end{tabular}

\section{CONTRIBUTIONSOFAUTHORS}

Janine Archer (JA) led the writing of the protocol and the review, assessed studies for inclusion, conducted content and quality data extraction and 'Risk of bias' assessments.

Peter Bower (PB) supervised and co-wrote each draft of the protocol and review and led the outcome data extraction, analyses and write-up of results.

Simon Gilbody (SG) supervised and commented on each draft of the protocol and review, provided consultation for queries where study inclusion was unclear and was consulted on analytical procedures.

David Richards (DR) commented on each draft of the protocol and review, provided consultation for queries where study inclusion was unclear, conducted content and quality data extraction and 'Risk of bias' assessments.

Linda Gask (LG) commented on each draft of the protocol and review and conducted content data extraction.

Karina Lovell (KL) commented on each draft of the protocol and review and conducted content data extraction.

Peter Coventry (PC) commented on each draft of the review and conducted quality data extraction and 'Risk of bias' assessments.

Chris Dickens (CD) commented on each draft of the review and conducted quality data extraction and 'Risk of bias' assessments.

\section{DECLARATIONSOF INTEREST}

The authors have been/are involved in the conduct of trials of collaborative care in the UK funded by the Medical Research Council. PB is a paid consultant to the British Association of Counselling and Psychotherapy, but the authors have no additional financial or other conflicts of interest in the results of the present review or the outcomes of these trials. 


\section{SOURCES OF SUPPORT}

\section{Internal sources}

- University of Manchester, UK.

- NIHR School for Primary Care, University of Manchester, UK.

- NIHR Collaboration for Leadership in Applied Health Research and Care for Greater Manchester, UK.

\section{External sources}

- NIHR Cochrane Review Incentive Scheme 2012, UK.

\section{DIFFERENCES BETWEEN PROTOCOLANDREVIEW}

The methods for this review are based on the revised protocol published online in 2012 (Archer 2007), which is an update of the original protocol to take into account the most recent version of the Cochrane Handbook for Systematic Reviews of Interventions available to authors (Higgins 2011a).

In the protocol we intended to report on social function outcomes. However, a very wide variety of social outcome measures were reported, and there was a lack of clarity over their definition, scope, and comparability. It was therefore not possible to produce a rigorous synthesis in the time frame of the review. We have extracted social function outcomes and may report them in a later update of the review.

We had planned to undertake a series of exploratory analyses to examine the influence of various factors outlined in the Subgroup analysis and investigation of heterogeneity section and other individual study-level factors in predicting the magnitude and direction of outcomes. We had also planned to assess the significance of predictive factors (selected a priori and outlined above) in explaining between-study heterogeneity, as measured by the $\mathrm{I}^{2}$ statistic, according to the method proposed in Higgins 2004 . However, as detailed in Potential biases in the review process, we were unable to undertake these further exploratory analyses due to time constraints, but we envisage incorporating them in the next review update.

\section{INDEX TERMS}

\section{Medical Subject Headings (MeSH)}

*Interprofessional Relations; Anxiety [*therapy]; Case Management [*organization \& administration]; Depression [*therapy]; Patient Care Team [*organization \& administration]; Primary Health Care [organization \& administration]; Psychiatric Nursing; Psychiatry; Psychology; Randomized Controlled Trials as Topic; Standard of Care

\section{MeSH check words}

Adult; Female; Humans; Male 